\title{
Mechanistic insights into alpha-Synuclein neuronal toxicity: misfolding, serine phosphorylation and interactions with Rab GTPases
}

\author{
Dissertation \\ zur Erlangung des mathematisch-naturwissenschaftlichen Doktorgrades \\ "Doctor rerum naturalium" \\ der Georg-August-Universität Göttingen
}

vorgelegt von

Guowei Yin

aus Kunming, China

Göttingen, 2012 
Mitglied des Betreuungsausschusses (Referent): Prof. Dr. Christian Griesinger NMR-basierte Strukturbiologie, Max-Planck-Institut für biophysikalische Chemie

Mitglied des Betreuungsausschusses (Koreferent): Prof. Dr. Gerhard Braus

Mikrobiologie und Genetik, Georg-August-Universität Göttingen

Mitglied des Betreuungsausschusses: Prof. Dr. André Fischer

European Neuroscience Institute Göttingen

Mitglied des Betreuungsausschusses: Prof. Dr. Markus Zweckstetter

NMR-basierte Strukturbiologie, Max-Planck-Institut für biophysikalische Chemie

Tag der mündlichen Prüfung: 


\section{Affidavit}

I hereby declare that this dissertation has been written independently and with no other sources and aids than quoted.

Guowei Yin 


\begin{abstract}
Alpha-Synuclein $(\alpha S)$, a protein abundant in presynaptic neurons, has been found as the major component of Lewy bodies, the neuronal inclusion identified as the hallmark of Parkinson's disease (PD). $\alpha \mathrm{S}$ adopts intrinsically disordered conformation under physiological conditions and possesses a high propensity for assembling into oligomers and fibrils during the pathological process of PD. Although the pathology of PD is still elusive, several lines of evidence with respect to the neuronal toxicity, cellular physiology, and protein structure indicate that $\alpha \mathrm{S}$ plays an important role in the development of PD and affects the normal cellular functions. In this dissertation we study the molecular mechanisms of serine phosphorylation, the interaction with RabGTPases and the aggregates structure of $\alpha \mathrm{S}$.

Phosphorylation is a major posttranslational modification of $\alpha \mathrm{S}$. Approximately $90 \%$ of $\alpha \mathrm{S}$ in LB is phosphoylated at Ser129, but the pathological role of phosphorylation has still not been determined. Ser87 is also an important serine phosphorylation site located at NAC region. To understand the mechanisms of phosphorylation, we investigate phosphorylation kinetics and the effects of phosphorylation on $\alpha \mathrm{S}$ aggregation. Real-time NMR spectroscopy is employed to study the phosphorylation kinetics of the Ser87 and Ser129 of $\alpha \mathrm{S}$. With the aid of the residuespecific resolution provided by NMR spectroscopy we reveal that the three familial mutations A30P, E46K, and A53T do not change the phosphorylation kinetics of Ser87 but slightly modify the kinetics of Ser129. At the same time, under the conditions of our assay, we also identify that PLK2 and PLK3 are capable of phosphorylating $\alpha \mathrm{S}$ at Ser129 with high selectivity and efficiency. Furthermore, we find that phosphorylation
\end{abstract}


of Ser129 by PLK3 and the phosphorylation mimicking mutants (P128E/S129E and M127E/P128E/S129E) do not affect the aggregation propensity of $\alpha \mathrm{S}$.

Rab GTPases are important for the regulation of vesicle trafficking in eukaryotic cells. Rab1, Rab3a, and Rab8a were suggested to protect against $\alpha$ S-induced cellular toxicity in different model systems. Focusing on molecular mechanisms involved in the interplay between Rab proteins and $\alpha \mathrm{S}$, we firstly identify that both the GDP- and GppNHp-bound Rab8a bind to the C-terminus of $\alpha \mathrm{S}$. However, $\alpha \mathrm{S}$ has a higher affinity to Rab8a(GDP) than Rab8a(GppNHp). Secondly, the positively charged C-terminal peptide of Rab8a is found to reproduce the binding of full-length Rab8a to $\alpha \mathrm{S}$, suggesting that a charge complementation between the C-terminal of both proteins is important for the interaction. Furthermore, we have assigned Rab8a in the GDP-bound state and find that in addition to the C-terminus the Switch I region of Rab8a serves as a binding site for the $\alpha \mathrm{S} \mathrm{C}$-terminus. Since the conformation of switch regions is highly dependent on nucleotide binding, this finding elucidates the binding preference for the GDP-bound state. Furthermore, Rab8a enhances the aggregation of $\alpha \mathrm{S}$ in both oligomerization and fibril formation. Rab1b and Rab3a do not bind to $\alpha \mathrm{S}$ but they can also significantly enhance the fibril formation of $\alpha \mathrm{S}$. Taken together, our results demonstrate a tight interplay between $\alpha \mathrm{S}$ and Rab proteins at the molecular level.

Furthermore, $\alpha \mathrm{S}$ aggregates, namely oligomers and fibrils, are highly associated with PD pathology. We perform solution NMR based hydrogen/deuterium (H/D) exchange to detect the fibril core of E46K and A53T $\alpha \mathrm{S}$ fibrils. These two mutations do not cause drastic changes to the fibril core structures in comparison to $w t$ $\alpha \mathrm{S}$ and a conserved core region has been suggested for $w t-\alpha \mathrm{S}$ and the three familial mutants. Furthermore, we optimize the H/D exchange method to detect the conformation of compounds-stabilized $\alpha \mathrm{S}$ oligomers. Our results suggest that the compounds-stabilized oligomers do not contain strongly hydrogen-bonded regions. 


\section{Acknowledgements}

I would like to thank Prof. Dr. Christian Griesinger for offering me this invaluable opportunity to undertake my thesis in this department for providing excellent facilities, research environment and continuous supports.

I would like to thank Prof. Dr. Markus Zweckstetter for his direct supervision, illuminating discussions, facilitation of knowledge transfer, and critical judgment throughout my thesis.

I would like to thank Prof. Dr. Gerhard Braus, Prof. Dr. Andre Fischer from the GeorgAugust-Universität Göttingen for their membership of my thesis committee and helpful suggestions during the committee meeting.

I am very grateful to Dr. Stefan Becker, Karin Giller and Sebastian Wolff for their preparations of alpha-Synuclein samples and help in the wet lab. I would thank Gerhard Wolf and Kerstin Overkamp for the HPLC manipulation and the peptide synthesis respectively. I would also thank our secretaries Mrs. Silberer and Mrs. Breiner, our IT system administrators Dr. Dirk Bockelmann and Jürgen Arve for their daily supports.

I would like to extend my thanks to Prof. Dr. Aymelt Itzen and Prof. Dr. Roger Goody from Max-Planck-Institute of Molecular Physiology, Dortmund for the continuous supply of the Rab protein samples and helpful suggestions in the project.

I would like to thank Prof. Dr. Tiago Fleming Outeiro from University Medical Center Göttingen for the collaboration in the Rab project and for accepting the request to be a member of my thesis defense committee. I would also like to thank Sibylle Eisbach, Tomás Lopes da Fonseca in the Outeiro's lab for the enjoyable experience of collaboration and our many discussions. 
I would like to thank Prof. Dr. Hilal Lashuel, Katerina E. Paleologou, Martial K. Mbefo from EFPL, Lausanne, for the collaboration in the phosphorylation projects.

I would like to thank Dr. Dietmar Riedel and Gudrun Heim for recording the electron microscopy for me.

I would like to thank Sergey Ryazanov and María L. Orcellet for the nice collaborations and sharing the research experience. I would like to thank Dr. Min-kyu Cho and Dr. Hai-young Kim for introducing the projects to me at the beginning and sharing valuable experiences with me throughout the thesis process. I would like to thank Shengqi Xiang for the instruction of NMR experiments and wet lab and many helpful discussions. I would like to thank Dr. Nasrollah Rezaei-Ghaleh for help in biophysical analysis and NMR experiments. I would like to thank Rakhi Bajaj, Saskia Villinger and Han Sun for their help in my thesis writing.

I would like to thank my all the current and former colleagues in our department for their support and friendship.

I would like to also thank Prof. Micheal Hörner, Chiritina Bach, Christin Fischer and other persons in the GGNB team for their kind help and excellent organization of the course and academic activities.

Finally, I would like to thank my family. I would express my overwhelming gratitude to my parents for their untiring support and consideration. Thanks to my wife Yuhuan for her love and companionship, and for our son. Thanks finally to my grandfather who passed away last year for his lifelong encouragement and advice which will remain with me always. 


\section{Table of contents}

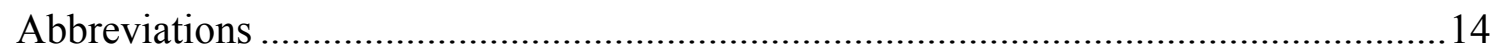

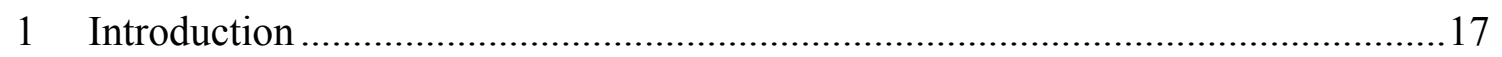

1.1 Parkinson's Disease and Synucleinopathies ........................................ 17

1.1.1 Parkinson's disease overview.................................................... 17

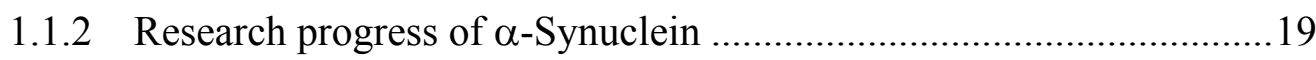

1.2 Intrinsically disordered proteins and protein misfolding ........................28

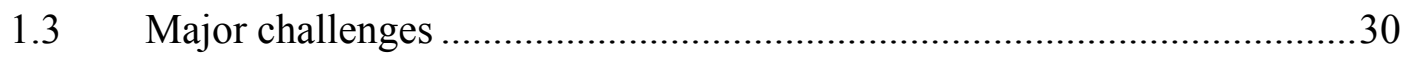

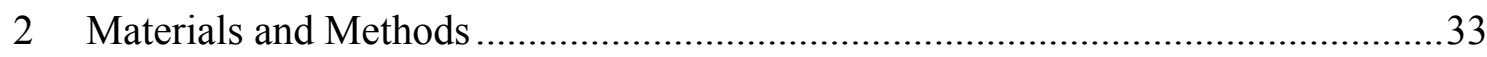

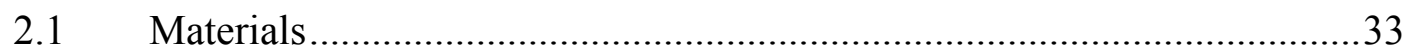

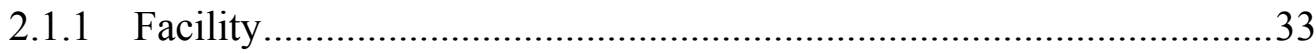

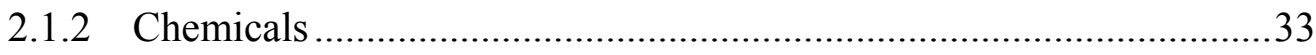

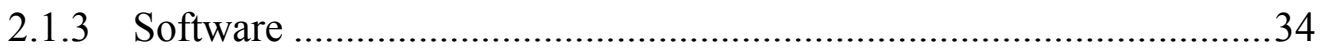

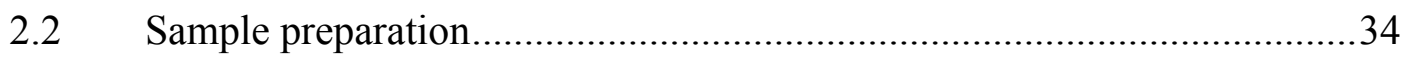

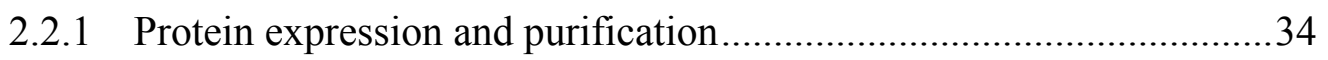

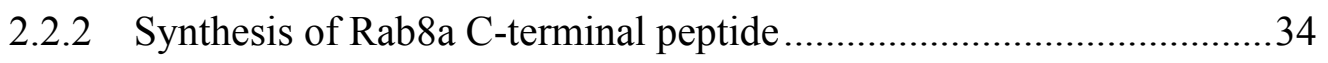

2.2.3 Preparation of the phosphorylated Ser129 $\alpha$-Synuclein ....................35

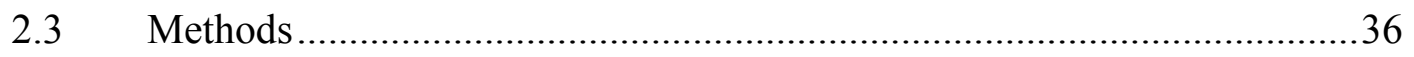

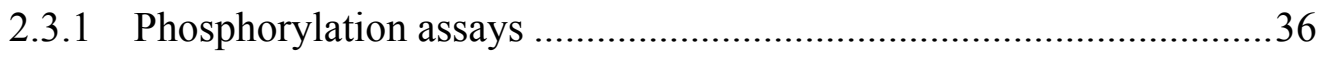

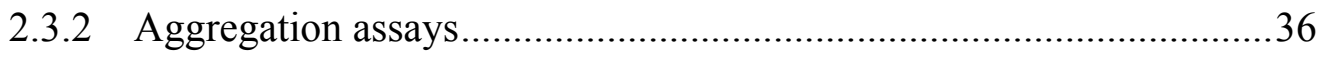


2.3.3 NMR spectroscopy

2.3.3.1 Backbone assignment

2.3.3.2 HSQC-based study of protein-protein/ligand interactions

2.3.3.3 Real-time NMR method used in the investigation of phosphorylation kinetics

2.3.3.4 NMR approach to study H/D exchange rate of protein backbone 42

2.3.3.5 Diffusion coefficient. 44

2.3.4 Biophysical methods .45

2.3.4.1 Fluorescence spectroscopy 45

2.3.4.2 UV-Vis Spectroscopy 46

2.3.4.3 Dynamic light scattering 46

2.3.4.4 Electron microscopy..... 47

2.3.4.5 Atomic force microscopy 47

3 Results 49

3.1 Phosphorylation of alpha-Synuclein....... 49

3.1.1 Phosphorylation of disease-associated $\alpha$-Synuclein mutations 49

3.1.1.1 Kinetics of $\alpha$-Synuclein phosphorylation by PLK2 50

3.1.1.2 Kinetics of $\alpha$-Synuclein phosphorylation by CK1 51

3.1.2 Phosphorylation of $\alpha$-Synuclein at Ser129 52

3.1.2.1 Kinetics comparison of different kinases (PLK1, PLK2, PLK3) 53

3.1.2.2 Aggregations assays of phosphorylated Ser129 $\alpha$-Synuclein ..... 57

3.1.2.3 Aggregation assays of Ser129 phosphorylation mimics of $\alpha$ Synuclein 61

3.2 Interplay between alpha-Synuclein and Rab proteins. 63

3.2.1 Sequence alignment of Rab1b, Rab3a and Rab8a 64 
3.2.2 Unique binding of Rab8a to $\alpha$-Synuclein .65

3.2.2.1 Rab8a, but not Rab1b and Rab3a binds $\alpha$-Synuclein ..................65

3.2.2.2 Anatomy of the interaction between $\alpha$-Synuclein and Rab8a .....67

3.2.2.3 C-terminally truncated Rab8a binds to $\alpha$-Synuclein 71

3.2.3 Aggregation assays of $\alpha$-Synuclein with Rab8a and it's C-terminus .74

3.2.4 Aggregation assays of $\alpha$-Synuclein with Rab1b and Rab3a ...............76

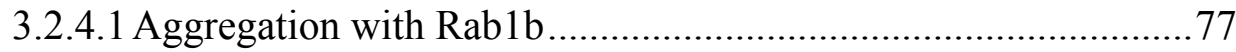

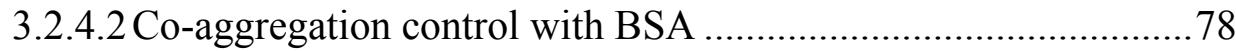

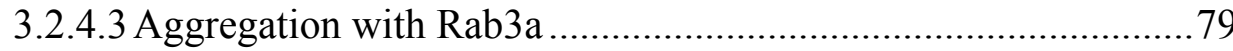

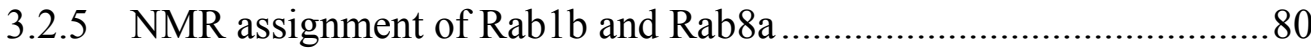

3.2.5.1 Assignment and structural analysis of Rab1b...........................81

3.2.5.2 Assignment and structural analysis of Rab8a ............................86

3.2.6 Mapping interaction sites on Rab8a .................................................90

3.3 Structural characterization of alpha-Synuclein aggregates ......................94

3.3.1 H/D exchange for fibrils of E46K and A53T $\alpha$-Synuclein mutants ...94

3.3.2 Probing conformation of the compounds-stabilized oligomers of

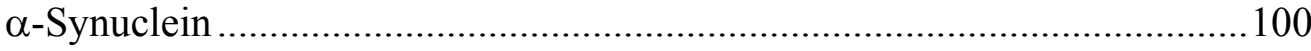

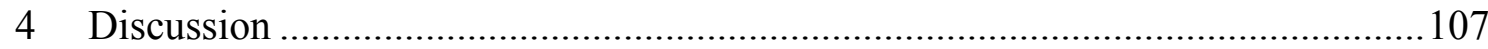

4.1 Functional relevance of alpha-Synuclein phosphorylation ..................... 107

4.1.1 The effect of PD-associated mutations on the phosphorylation of $\alpha$ Synuclein 107

4.1.2 Functional relevance of the Ser129 phosphorylation of $\alpha$-Synuclein 108

4.2 Rab proteins bind to alpha-Synuclein and modulate its aggregation ....... 110

4.2.1 Rab8a binds to monomeric $\alpha \mathrm{S}$ 
4.2.1.1 Rab8a binds to C-terminus of $\alpha \mathrm{S}$.....

4.2.1.2 $\alpha \mathrm{S}$ binds to the Switch I region of Rab8a

4.2.1.3 $\alpha \mathrm{S}$ binds to the C-terminus of Rab8a

4.2.1.4 The Ser129 phosphorylation enhances the binding of $\alpha \mathrm{S}$ to Rab8a 115

4.2.1.5 Overview for the molecular interaction of $\alpha \mathrm{S}$ and Rab8a

4.2.2 Rab proteins modulate the $\alpha \mathrm{S}$ aggregation

4.2.2.1 Rab8a modulates $\alpha \mathrm{S}$ aggregation

4.2.2.2 Rab1b and Rab3a modulate $\alpha \mathrm{S}$ aggregation

4.2.2.3 Pathological implications of Rabs modulating $\alpha \mathrm{S}$ aggregation 120

4.3 Conserved fibril core and its implications to the conformation of alphaSynuclein oligomers 121

4.3.1 Molecular structure and supermolecular assembly of $\alpha \mathrm{S}$ fibrils 121

4.3.2 Structural relationship of $\alpha \mathrm{S}$ fibrils and oligomers and the prospective for structural study of $\alpha \mathrm{S}$ oligomers 125

4.4 Structural and functional importance of alpha-Synuclein C-terminus .... 128

5 Summary and outlook 131

6 References 133

Appendix 157

I. Backbone assignment of Rab1b(1-175)-GDP 157

II. Backbone assignment of Rab8a-GDP 161

Publications 166 


\section{Abbreviations}

\begin{tabular}{|c|c|}
\hline$\alpha \mathrm{S}$ & Alpha-Synuclein \\
\hline ATP & Adenosine-5'-Triphosphate \\
\hline AmppNHp & 5'-Adenylylimidodiphosphate \\
\hline BEST & Band-selective Excitation Short-Transient \\
\hline BSA & Bovine serum albumin \\
\hline CK1 & Casein kinase 1 \\
\hline CNS & Central nervous system \\
\hline CSA & Chemical shift anisotropy \\
\hline DA & Dopamine \\
\hline DMSO & Dimethyl sulfoxide \\
\hline DOSY & Diffusion ordered spectroscopy \\
\hline DTT & Dithiotreitol \\
\hline DLS & Dynamic light scattering \\
\hline E. coli & Escherichia coli \\
\hline ER & Endoplasmic reticulum \\
\hline GDP & Guanosine diphosphate \\
\hline GTP & Guanosine triphosphate \\
\hline GuSCN & Guanidinium thiocyanate \\
\hline $\mathrm{H} / \mathrm{D}$ & Hydrogen/Deuterium \\
\hline HSQC & Heteronuclear single quantum coherence \\
\hline HPLC & High performance liquid chromatography \\
\hline IPAP & In-phase-anti-phase \\
\hline $\mathrm{hr}$ & hour \\
\hline
\end{tabular}


HSPs

INEPT

$\mathrm{K}_{\mathrm{d}}$

LB

LRRK2

NAC

NMR

PD

PDB

PLKs

PRE

pS129

Rab1b- $\delta \mathrm{C}$

Rab8a- $\delta \mathrm{C}$

$\mathrm{Rh}$

RDC

SOFAST

ThT

TROSY

$w t$
Heat shock proteins

Insensitive nuclei enhanced by polarization transfer

Dissociation constant

Lewy body

Leucine-rich repeat kinases 2

Non-A $\beta$ component of Alzheimer's disease amyloid plaques

Nuclear magnetic resonance

Parkinson's disease

Protein data bank

Polo-like kinases

Paramagnetic relaxation enhancement

Phsphorylated Ser129

C-terminally truncated Rab1b (1-175)

C-terminally truncated Rab8a (6-176)

Hydrodynamic radius

Residual dipolar coupling

band-Selective Optimized-Flip-Angle Short-Transient

Thioflavin-T

Transverse relaxation optimized spectroscopy

wild-type 



\section{Introduction}

"Involuntary tremulous motion, with lessened muscular power, in parts not in action and even when supported; with a propensity to bend the trunk forwards, and to pass from a walking to a running pace: the senses and intellects being uninjured."

- James Parkinson, “An Essay on the Shaking Palsy”, 1817.

The symptoms of what we now call Parkinson's disease were first described by the English apothecary surgeon James Parkinson in 1817 and named "maladie de Parkinson" or Parkinson's disease (PD) in 1861 by Jean-Martin Charcot, the founder of modern neurology. In today's aging society, PD is the second most common neurodegenerative disease after Alzheimer disease and approximately 7-10 millions people worldwide are living with PD (Statistics from Parkinson's disease foundation, "PDF").

In section 1.1, I will open with an overview of the Parkinson's disease and synucleinopathies will be exerted following the order "from bedside to bench" and accordingly the motivations of the research undertaken in this dissertation will be addressed. The focus of this dissertation upon protein $\alpha$-Synuclein $(\alpha \mathrm{S})$ as one major cause of PD will be introduced in section 1.1.1 and 1.1.2 in terms of its pathological, biophysical, and structural characteristics. In section 1.2, the category of intrinsically disordered proteins (IDPs) will be described particularly in terms of its general structural features and the propensity of protein misfolding. To conclude this chapter, within the scope of this dissertation, the challenges for current research of PD pathologies with respect to $\alpha$-Synuclein will be listed in section 1.3.

\subsection{Parkinson's Disease and Synucleinopathies}

\subsubsection{Parkinson's disease overview}

The occurrence of Parkinson's disease is strongly age-dependent (Lees et al, 2009). The average age of onset is about 65 years old and the incidence rises from $0.6 \%$ 
at age 65-69 years old to $2.6-3.5 \%$ at age $85-89$ years old (de Rijk et al, 2000). The onset of Parkinson's disease commonly presents with one or a few of the following motor-symptoms: impairment of dexterity, slight dragging of one foot, fatigue, stiffness, lugubrious stiff face, hangdog appearance, flexion of one arm with lack of swing, monotonous quality to speech, extreme slowing down (Hardy et al, 2009). These early symptoms are usually difficult to notice for patients and a delay of 2-3 years from first symptoms to diagnosis is common. Nonmotor symptoms (NMS) such as depress pain, hyposmia (loss of sense of smelling), disturbed sleep, and dysautonomia start to increase progressively in frequency and severity (Chaudhuri et al, 2006). In the late sages, Parkinson's disease is characterized by a masked and expressionless face, the monotonous, festinant, and slightly slurred speech, flexed simian posture with a severe pill rolling tremor of the hands (Hardy et al, 2009). Constipation, chewing and swallowing difficulties, drooling of saliva, and urge urinary incontinence are concomitant features.

The primary pathology of Parkinson's disease is progressive dopamingernic neuron loss in the substantial nigra of the midbrain. Several genes such as SNCA (PARK1), PARK2 (PARK2), PINK1 (PARK6), PARK7 (PARK7), LRRK-2(PARK8) have been implicated in this pathology, where the protein products of these genes are $\alpha$ Synulcein, PARKIN, Serine/threonine-protein kinase PINK1, DJ-1, and Leucine-rich repeat serine/threonine-protein kinase 2 (Pankratz et al, 2004). Since 2009, a large-scale GWAS genomic screen has been applied to PD and several novel putative PDassociated genes have been identified as a marked progress in genome-wide studies of PD (Hardy, 2010; Nalls et al, 2011; Satake et al, 2009; Simon-Sanchez et al, 2009).

The dominant mechanism associated with dopaminergic neuronal loss is the Lewy body pathology. The Lewy body (LB), the hallmark of PD, is one type of neuronal inclusion deposited in the disease-associated neurons and is viewed as the cause of neuron death. The major component of LB are the amyloid fibrils formed through the aggregation of protein $\alpha$-Synulcein $(\alpha \mathrm{S})$ (Spillantini et al, 1997), the protein product of the SNCA gene (Polymeropoulos et al, 1997). Therefore, it is crucial to study the roles of $\alpha \mathrm{S}$ in the pathological scenario of PD in terms of its biochemical and biophysical natures, aggregation pathway, toxic mechanisms, pathological regulation factors et al. 
This is the original motivation of this dissertation. Moreover, $\alpha \mathrm{S}$ is not only present in $\mathrm{PD}$, rather it also gets deposited into oligodendroglial cytoplasmic inclusions in multiple system atrophy (MSA) and into large axonal spheroids in a number of rarer neuroaxonal dystrophies (Halliday et al, 2011; Spillantini \& Goedert, 2000). Thus, these 3 diseases caused by the abnormal inclusion formed by $\alpha \mathrm{S}$ are grouped under the single category of synucleinopathies.

To date, PD is incurable, but oral drug administration improves the life quality and functional capacity of patients. The major PD treatment is dopamine replacement therapy which aims to increase the level of dompamines in the brain to compensate for the decreased levels caused by the neuronal loss. Levodopa (L-dopa) is one of most efficient and widely used drugs because it is the precursor of dopamine and able to pass through blood brain barrier (BBB) (Schapira et al, 2006). The catechol-Omethyltransferase (COMT) inhibitors and monoamine oxidase (MAO) inhibitors are two main drugs that also reduce the metabolism of L-dopa (Huynh, 2011), whilst ergot dopamine agonists are in another class of PD drugs that delay early onset of motor complication with high efficiency (Schapira et al, 2006). However, the application of these agents can not stop the dopaminergic neuron loss and the neurdegerative disorder and current drugs in markets and in clinical trials targeting $\alpha \mathrm{S}$ are rare (Huynh, 2011; Schapira et al, 2006). Recent studies indicate that the inhibitors of COMT, entacapone and tolcapone, are able to block the fibril formation of $\alpha \mathrm{S}$ (Di Giovanni et al, 2010). In July 2012, it was reported that the first therapeutic antibody of PD designed to clear $\alpha \mathrm{S}$ stocked in the membranes and spread between the cells has been under clinical trial (Dolgin, 2012). This antibody has been proved to reduce the neuronal loss and improve the cognition in the transgenic mouse models and trials on human have now begun.

\subsubsection{Research progress of $\alpha$-Synuclein}

\section{Primary sequence of $\alpha$-Synuclein}

$\alpha \mathrm{S}$ is an intrinsically disordered protein comprising of 140 amino acids (Polymeropoulos et al, 1997) that are mainly divided into 3 fundamental domains: Nterminal (1-60), NAC (61-95) and C-terminal (96-140) (Bisaglia et al, 2009) [Figure 1.1]. The N-terminal is amphipathic with high affinity to membrane. The middle NAC 
region (non amyloid component) is a highly hydrophobic part of the protein and is located in the core region of amyloid fibrils, which is crucial in fibril formation. There are seven imperfect 11-mer repeats (XKTKEGVXXXX) spread through the N-terminal to NAC region. The total number and the conserved motif "KTKEGV" of these imperfect repeats have been implicated in lipid-binding (Davidson et al, 1998)and regulating aggregation (Kessler et al, 2003). The $\mathrm{C}$-terminal is acidic which is enriched in negatively charged residues with a highly disordered conformation. 3 familial pathological mutations A30P, E46K and A53T identified in the different kindred are located at the N-terminal and are highly involved in functional and pathological modification.

\section{Protein structures and biophysics of $\alpha$-Synuclein aggregation}

Structural insights into $\alpha \mathrm{S}$ monomers reveal that $\alpha \mathrm{S}$ is intrinsically disordered but not fully denatured. High resolution NMR data indicates that $\alpha \mathrm{S}$ monomer possesses the transient long range interactions between $\mathrm{N}$-terminal and $\mathrm{C}$-terminal of $\alpha \mathrm{S}$ and thereby stabilizes tertiary structure of the protein (Bertoncini et al, 2005b; Dedmon et al, 2005c). The structural ensembles of $\alpha \mathrm{S}$ mapped by the paramagnetic relaxation enhancement measured by solution NMR indicate that the long-range interactions close the protein conformation and lock the hydrophobic NAC region into a state less accessible for the $\mathrm{NAC}$ of neighboring $\alpha \mathrm{S}$, which is considered as the auto-inhibition mechanism of the aggregation (Bertoncini et al, 2005b). Later studies reveal that the electrostatic interactions between two termini of $\alpha \mathrm{S}$ also contributed to the intermolecular interactions between the N-terminal of one $\alpha \mathrm{S}$ and the C-terminal of another in a headto-tail manner ( $\mathrm{Wu} \& \mathrm{Baum}$ ), which is in concert with the in vivo observation by bimolecular fluorescence complementation(BiFC) (Outeiro et al, 2008). Recent studies have suggested that the endogenous $\alpha \mathrm{S}$ directly isolated from the human living cells was mainly present as the helically folded tetramer (Bartels et al, 2011). The authors discussed that the protein recombination techniques and harsh conditions of sample purification for $\alpha \mathrm{S}$ commonly adopted in the labs (Weinreb et al, 1996) might be harmful to to the retention of the native folded conformation of $\alpha \mathrm{S}$. However, a later study used the same conditions to generate the $\alpha \mathrm{S}$ by isolating it from the living cells and found that the generated $\alpha \mathrm{S}$ was still dominantly disordered in comparison to the 
sample obtained through common recombination and purifications (Fauvet et al, 2012b). Thus the accumulated evidence confirms that the physiological conformation of $\alpha \mathrm{S}$ should be disordered (Bertoncini et al, 2005b; Conway et al, 1998; Weinreb et al, 1996), but the $\alpha$-helix propensity of $\alpha \mathrm{S} N$-terminus enables the protein to form the helix conformation under the certain conditions i.e. membrane binding.

The intrinsic disorder and the presence of highly hydrophobic NAC region determine a strong aggregation propensity of $\alpha \mathrm{S}$. The aggregation of $\alpha \mathrm{S}$ can be generally divided into 3 phases: oligomerization, fibril growth and saturation (Fink, 2006). The aggregation pathway starting from $\alpha \mathrm{S}$ monomers to the final fibrils deposited in the LB in plaques of PD patient brain is demonstrated in Figure 1.1.

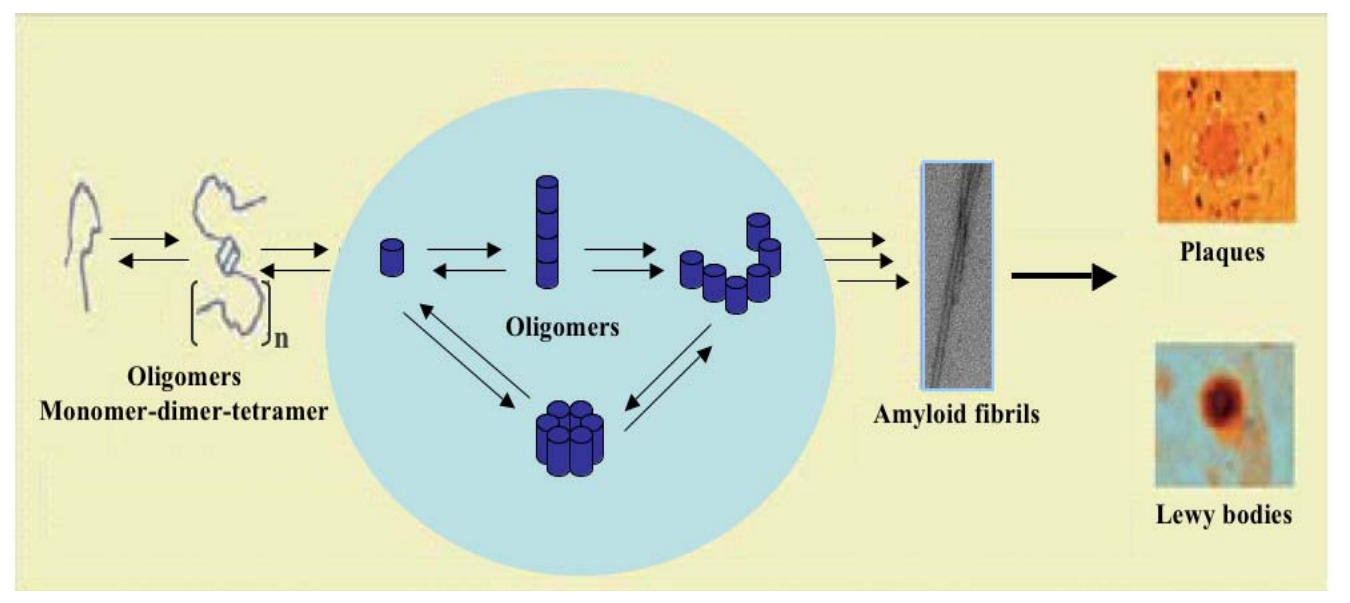

Figure 1.1 Aggregation pathway of $\alpha$-Synuclein. The flowchart demonstrates the aggregation process of the oligomerization, fibrillization and deposition of LB (dark dots) observed at PD plaques. The figure is taken from (Lashuel \& Lansbury, 2006).

The disordered $\alpha \mathrm{S}$ monomers firstly associate with each other to form the oligomeric species. This process is kinetically defined as the nucleation period which is the rate determinating step for the whole aggregation process. The structural study of $\alpha \mathrm{S}$ oligomers is of major interests and poses major challenges to understand $\alpha \mathrm{S}$ neuronal toxicity. $\alpha \mathrm{S}$ oligomers represent distinct shapes i.e. chain-like or annular observed by electron microscopy (EM) and atomic force microscopy (AFM) (Lashuel \& Lansbury, 2006). The secondary structures of $\alpha \mathrm{S}$ oligomers were indicated by CD as the $\beta$-sheet rich conformations. However, due to the nature of the aggregation intermediates i.e. structural heterogeneity and weak stability etc., $\alpha \mathrm{S}$ oligomers until 
now are still not characterized with high resolution. A recent study suggests that within the process of the oligomerization there is one conversion from the smaller sized oligomers to large sized oligomer and that the larger oligomers are toxic (Cremades et al, 2012). Moreover, SAXS data suggests that $\alpha \mathrm{S}$ oligomers are finally assembled into fibrils with conserved size parameters, implying that mature $\alpha \mathrm{S}$ oligomers act as the building blocks of $\alpha \mathrm{S}$ fibrils (Giehm et al, 2011b).

As shown in figure 1.2, after the oligomerization, $\alpha \mathrm{S}$ is finally assembled into the fibrils with well-defined morphorlogys. The phase from the ending of oligomerization to the final plateau phase is fibril growth which is kinetically defined as elongation. In this phase, the oligomers of $\alpha \mathrm{S}$ are assembled into the protofilaments along the fibril axis direction and the protofilaments are further associated with each other to form the morphologically straight bundle or the twisted fibril. Since the amyloid fibrils of $\alpha \mathrm{S}$ are stable and insoluble in water, they are readily separated and purified for further structural analysis. Therefore, the structures of the amyloid core region of $\alpha \mathrm{S}$ have been well studied by high resolution methods such as ssNMR, solution NMR based H/D exchange, Mass spectrum, EPR et al (Chen et al, 2007; Cho et al, 2011; Comellas et al, 2011; Del Mar et al, 2005; Heise et al, 2008; Heise et al, 2005; Lemkau et al, 2012; Vilar et al, 2008). The boundaries of the fibril core region have been measured, where the core region has been extended from the NAC to $\sim 37-97$. However, there are discrepancies amongst the different reports regarding the locations and lengths of $\beta$-strands, involving factors such as sample preparation, the polymorphism of fibrils and different technique details of the experiments which should be taken into account to address the differences. Besides the fibril core, two termini of $\alpha \mathrm{S}$ are mainly disordered. A recent report suggests that part of $\mathrm{N}$-terminal residues are experiencing contact with the core region and are likely to be participating in the fibril formation (Lemkau et al, 2012). The C-terminal of $\alpha \mathrm{S}$ still remains in a highly disordered nature in the fibrillar state. 


\section{Posttranslational modifications and phosphorylation}

There are several posttranslational modifications (PTMs) such as phosphorylation, truncation, ubiquitination, nitration, and acetylation associated with LB formation in PD patient brains and transgenic animal models (Oueslati et al, 2010a). The effects of these PTMs of $\alpha \mathrm{S}$ on the protein structure, aggregation propensity, neuronal toxicity and disease progression have not been determined. Most of PTMs are located at the Cterminus of $\alpha \mathrm{S}$. Phosphorylation is one major PTM of $\alpha \mathrm{S}$. Multiple sites such as Ser87, T92, Y125, S129, Y133, Y136 have been reported to be phosphorylated by the known kinases like CKI, PLKs, GRKs, LRRK2 et al. Truncation is another major PTM of $\alpha \mathrm{S}$. In LBs, the major truncation sites at the C-terminus are D115, D119, N122, Y133 and D135 (Anderson et al, 2006). Accumulating evidence suggests that the C-terminal truncations of $\alpha \mathrm{S}$ enhance aggregation propensity and initiate the aggregation process by seeding both in vivo and in vitro(Hoyer et al, 2002; Li et al, 2005; Liu et al, 2005). The co-localization of ubiquitin with $\alpha \mathrm{S}$ has been extensively found in LBs and Lewy neutrites (Gomez-Tortosa et al, 2000). The K12, K21 and K23 of $\alpha \mathrm{S}$ have been identified as major sites responsible for ubiquitination in LBs (Anderson et al, 2006). In addition, other lysine residues are also able to be ubiquitinated in vitro and in cell cultures, which most of them are located at the N-terminus of $\alpha \mathrm{S}$ (Nonaka et al, 2005; Rott et al, 2008). But the relationship between ubiquitination and degradation of misfoled $\alpha \mathrm{S}$ by ubiquitin-mediated proteasome pathway is still elusive (Tofaris et al, 2001; Tofaris et al, 2003). A recent study indicates that ubiquitination by Nedd4 ligase promotes $\alpha \mathrm{S}$ degradation by the endosomal-lysosomal pathway (Tofaris et al, 2011). Nitration is a potential mediator of PD and other neurodegenerative diseases (Souza et al, 2000). There are 4 tyrosine residues, Y39, Y125, Y133 and Y136, which can be nitrated in $\alpha \mathrm{S}$. The selectivity and specificity of different tyrosines is linked to the onset and progression of synucleinopathies (Giasson et al, 2000). N-terminal acetylation of $\alpha \mathrm{S}$ has been less studied than other PTMs mentioned above and only recently has been focused on its effects on protein conformation, lipid-binding, aggregation propensity (Bartels et al, 2011; Fauvet et al, 2012a; Kang et al, 2012; Maltsev et al, 2012).

Phosphorylation is the dominant PTM and the phosphorylation sites at Cterminal are highly conserved through the different species [Figure 1.2] (Hejjaoui et al, 
2012; Oueslati et al, 2010a). Of the Ser, Tyr and Thr phosphorylation, Ser phosphorylation has been extensively studied due to its prevalence in PD. Approximately $90 \%$ of $\alpha \mathrm{S}$ has been identified as phosphorylated at Ser129 of $\alpha \mathrm{S}$. However, there are several key issues with respect to this phosphorylation which remain elusive: 1) which kinases are responsible for regulating this phosphorylation; 2) what is the consequence of this phosphorylation on $\alpha \mathrm{S}$ structure in vivo; 3) whether this phosphorylation facilitates, inhibits or has no affect on $\alpha \mathrm{S}$ aggregation; 4) dose phosphorylation occur before or after $\alpha \mathrm{S}$ aggregation and LB formation; 5) how does this phosphorylation modulate the neuronal toxicity of $\alpha \mathrm{S}$. Several kinases are involved in the Ser129 phosphorylation. Polo-like kinase (PLKs), G protein-coupled receptor kinases (GRKs), LRRK2, and Casein kinase II (CKII) are reported to specifically phosphorylate $\alpha \mathrm{S}$ at Ser129 (Oueslati et al, 2010a). CKI is a kinase that is able to phosphorylate $\alpha \mathrm{S}$ at both Ser87 and Ser129. NMR studies point out that phosphorylation of Ser87 and Ser129 together or individually can extend the conformation of $\alpha \mathrm{S}$ in vitro (Paleologou et al, 2010; Paleologou et al, 2008). The phosphorylation of Ser87 and its mutation mimics exclusively inhibit the fibril formation of $\alpha \mathrm{S}$ (Paleologou et al, 2010). But at Ser129, the major phosphorylation site, the effect of phosphorylation at this residue on $\alpha \mathrm{S}$ aggregation still needs to be determined. Recent studies indicating that $\alpha \mathrm{S}$ fibrils are able to be phosphorylated at Ser129 in vitro and that this phosphorylation is likely independent of aggregation, suggesting that this modification can occur after aggregation (Mbefo et al, 2010; Waxman \& Giasson, 2011). One central issue is to investigate the effects of phosphorylation on aggregation behavior and toxicity of $\alpha \mathrm{S}$ in vivo. However, the related results obtained from different animal models present large diversity, which due to the model difference and partially due to the mimics can not completely reproduce the effects of real phosphorylation (Braithwaite et al, 2012; Oueslati et al, 2010a). Interestingly, in the SH-SY5Y cell model, it's suggested that Ser129 phosphorylation activates ER stress by inducing unfolded protein responses (UPRs) which is possibly caused by the disruption of ER-Golgi trafficking (Sugeno et al, 2008). On the other hand, Ser129 phosphorylation is suggested to suppress $\alpha \mathrm{S}$-induced vesicle trafficking deficit depending on genetic context in yeast model (Sancenon et al, 2012). These two 
findings together suggest that Ser129 phosphorylation possesses a potential role in regulating vesicle trafficking.

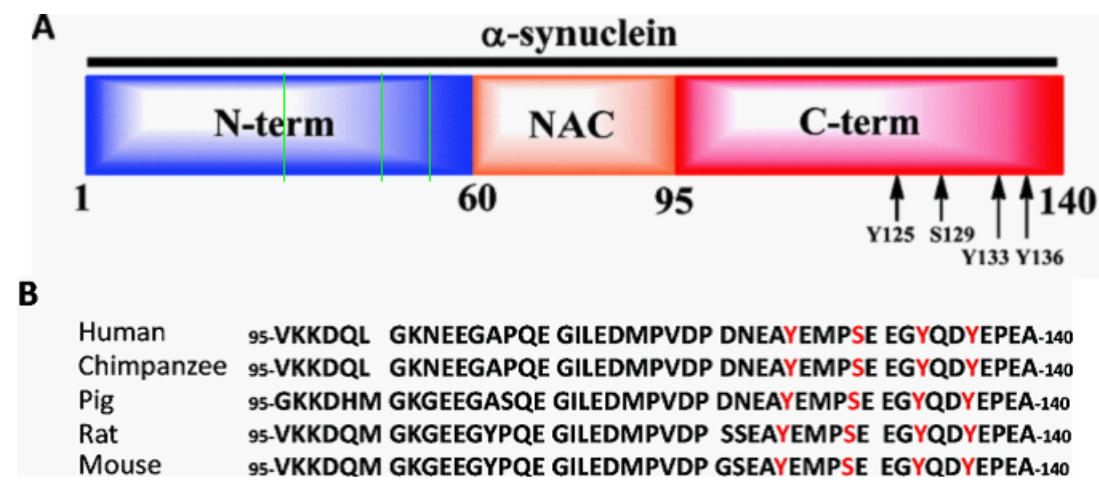

Figure 1.2 Primary structure of $\alpha \mathrm{S}$. (A) Schematic depiction of $\alpha \mathrm{S}$ sequence with its 3 domains. The positions of 3 familial mutations at the N-terminal are indicated by green lines. Identified and putative phosphorylation sites at C-terminal are indicated by black arrows. (B) Sequence analysis for the Cterminal from 5 different species indicates that the phosphorylation sites are highly conserved. Figures are taken from (Hejjaoui et al, 2012).

\section{Alpha-Synuclein affects vesicle trafficking and its interplay with Rab-GTPases}

Several lines of evidence suggest that $\alpha \mathrm{S}$ is involved in the vesicle trafficking in synaptic neurons. $\alpha \mathrm{S}$ was reported to assist synaptic vesicle recycling and neurotransmitter release (Ben Gedalya et al, 2009; Liu et al, 2004). $\alpha \mathrm{S}$ ameliorated the neurodegeneration caused by the depletion of cysteine-string protein-alpha (CSPalpha), which suggests it has a positive role in co-operation with CSPalpha and SNAREs (Chandra et al, 2005). Moreover, it has been suggest that $\alpha \mathrm{S}$ has a role in the maintenance of synaptic vesicle pools (Cabin et al, 2002; Murphy et al, 2000), activitydependent dopamine release (Abeliovich et al, 2000) and as a negative regulator of the vesicle priming (Larsen et al, 2006). However, more commonly, altering the expression levels and biophysical properties of $\alpha \mathrm{S}$ and its familial mutants leads to the deficits of vesicle trafficking at multiple steps in animal models (Auluck et al, 2010).

Rab-GTPases play a key role in coordinating both vesicle formation and trafficking (Stenmark, 2009). Serveral Rab proteins have been implicated in directly or indirectly interactions with $\alpha \mathrm{S}$ in PD models. Immunoprecipitation suggests Rab3a binding to $\alpha \mathrm{S}$ aggregates in LBs (Dalfo et al, 2004a). At same time, Rab3a, Rab5 and 
Rab8 were reported to coimmunoprecipitate with $\alpha \mathrm{S}$ in $\mathrm{A} 30 \mathrm{P} \alpha \mathrm{S}$ transgenic mice (Dalfo et al, 2004b). In PD models, $\alpha \mathrm{S}$ overexpression disrupted vesicle trafficking between the ER and Golgi and overexpression of Rab1 attenuated $\alpha \mathrm{S}$ toxicity (Cooper et al, 2006). Furthermore, Rab homeostasis was generally disturbed by $\alpha \mathrm{S}$ in yeast and the overexpression of Rab1, Rab3a and Rab8a was protective against $\alpha$ S-induced toxicity in 3 different animal models (Gitler et al, 2008). $\alpha \mathrm{S}$ was also reported to induce aggregation of many yeast Rab GTPases in the yeast model (Soper et al, 2011). In addition, Rab11a together with HSP90 displayed a regulatory role in the recycling of extracellular $\alpha \mathrm{S}$ (Liu et al, 2009). Moreover, overexpression of Rab3b, an isoform of Rab3, protected dopaminergic neurons in the PD model by improving dopamine handling and storage capacity at presynaptic terminals (Chung et al, 2009). These findings suggest extensive interactions between Rab proteins and $\alpha \mathrm{S}$ and Rab proteins generally protect against vesicle trafficking deficits in neuronal models of synucleinopahy.

\section{Cellular toxicity}

Gain of toxic functions through protein misfolding/aggregation is one unifying feature of neurodegenerative diseases i.e. Alzheimer's disease, PD etc. (Lansbury \& Lashuel, 2006; Stefani \& Dobson, 2003). But which is the major toxic species of $\alpha \mathrm{S}$ - monomers, oligomers or fibrils? This is a central question to be addressed in the research into the the synucleinopathy in PD. In the last ten years, a significant amount of evidence has developed which suggests that the most toxic species of $\alpha \mathrm{S}$ and $\mathrm{A} \beta$ is the pre-fibrillar species (oligomers) rather than the insoluble neuronal deposition i.e. mature fibrils, LBs; whereas the well-formed amyloid fibrils are less harmful or even protective (Caughey \& Lansbury, 2003; Cookson, 2009; Cookson \& van der Brug, 2008; Lashuel et al, 2002a; Lashuel et al, 2002b; Volles \& Lansbury, 2003; Winklhofer et al, 2008). One prevalent hypothesis attributes the basis of the oligomeric toxicity to the high membrane affinity of the soluble species of oligomers with respect to the membrane distortion and membrane leakage caused by the pore-forming capacity of the oligomers (Auluck et al; Bodner et al, 2009; Bodner et al, 2010; Butterfield \& Lashuel; Lashuel \& Lansbury, 2006). In our lab, the pore-forming propensities have been well-characterized in vitro by generating the aggregation on-pathway oligomers from the cold-dissociation of 
mature $\alpha \mathrm{S}$ fibrils (Kim et al, 2009) and the oligomers of triple proline $\alpha \mathrm{S}$ mutant designed on the basis of NMR structure (Karpinar et al, 2009). However, the oligomeric species normally presents continuum distribution in size and molecular weights, structural heterogeneity, instability and the mixture with $\alpha \mathrm{S}$ monomers that make oligomers difficult to be separated. Therefore, we are still lacking the high resolution structural information of $\alpha \mathrm{S}$ oligomers that determine its critical mechanism in the scenario of $\alpha \mathrm{S}$ cellular toxicities.

A concomitant question is whether we can exclude the cellular toxicities of $\alpha \mathrm{S}$ fibrils with the dominant assumption that the oligomeric species are most toxic. However, a consensus upon the role of $\alpha \mathrm{S}$ fibrils has still not achieved. Recent studies have reported that exogenous fibrils of $\alpha \mathrm{S}$ were transmitted into cells and thus seeded intra-cellular aggregation and prompted LB formation (Li et al, 2008; Luk et al, 2009). This mechanism of fibril transmission can then lead to synaptic dysfunction and neuron death (Volpicelli-Daley et al). Furthermore, this transmission was also observed between the affected neurons and the grafted neurons in both clinical trials and animal models (Hansen et al; Li et al, 2008). Thus, the mechanisms of $\alpha \mathrm{S}$ cellular toxicity still need to exact and systematic determination. Meanwhile, two relatively novel conceptions have been emerging: 1) the prion-like transmission of the pathological aggregates propagate the synucleinopathy between cells, different brain regions and organs, where the transmissions are realized by exo/endocytosis, membrane leakage, passive membrane translocation or alternatively by exosomal release or transport along tunnelling nanotubes (Angot et al, 2010; Braak et al, 2002; Brundin et al; Brundin et al, 2010; Hawkes et al, 2007; Pan-Montojo et al, 2010; Steiner et al) [Figure 1.3]; 2) the aggregation process is toxic in comparison with the single species (monomers or oligomers, fibrils) (Taschenberger et al, 2012). 


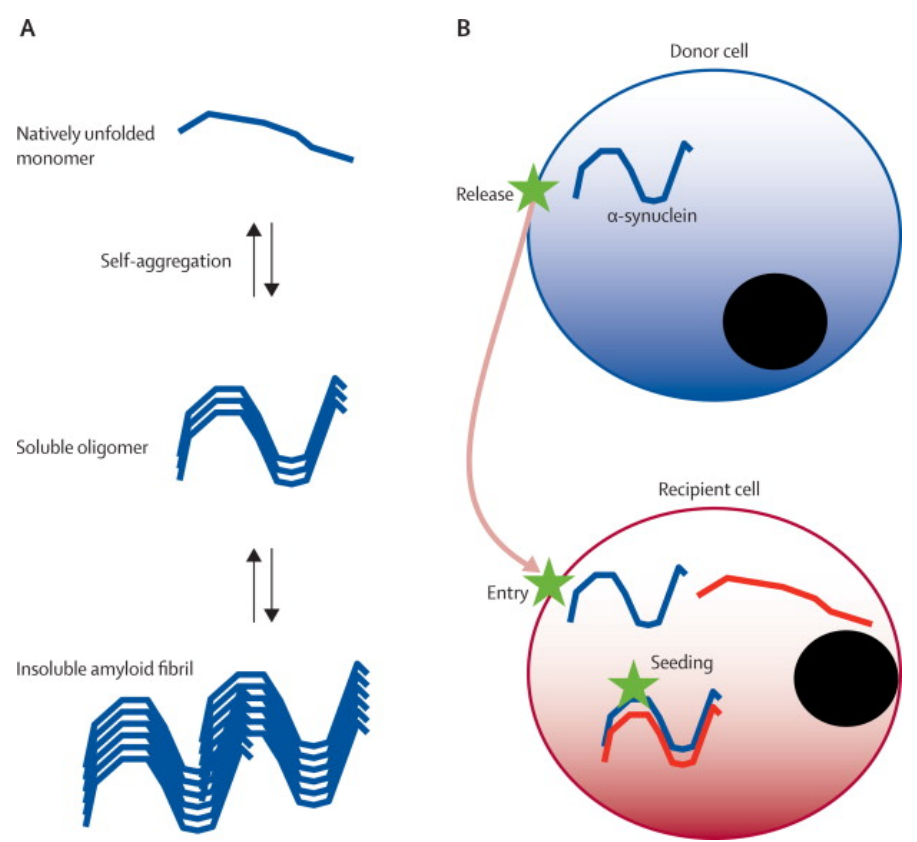

Figure 1.3 Putative mechanisms of $\alpha$-synuclein prion-like propagation. (A) The different species of $\alpha$-synuclein coexist in a highly dynamic equilibrium. (B) The prion-like transmission of $\alpha \mathrm{S}$ follows an order that donor cell - the extracellular space -recipient cell. Figures are taken from (Angot et al, 2010)

\subsection{Intrinsically disordered proteins and protein misfolding}

Intrinsically disordered proteins (IDPs) are one category of proteins which are not folded in their functional form (Jensen et al, 2009). But differently from other unstructural proteins, IDPs possess the possibility of transient long-range and local order and the possibility of folding after binding to the partners (Jensen et al, 2009; Rezaei-Ghaleh et al, 2012). IDPs have been shown to participate in a broad range of cellular processes and the development of several human pathologies like neurodegenerative disease and cancer (Dyson \& Wright, 2005).

IDPs present high structural flexibility and thus the traditional structural methods established on precise determination of well-defined protein structures are not suitable for IDPs (Jensen et al, 2009). In addition, X-ray crystallography is not applicable to IDPs due to their disordered nature. Rather than the determination of a single set of atomic coordinates, therefore, the conformational behaviors of protein backbone or an explicit ensemble description of interconverting structures are more appropriate for the structural description of IDPs. For this, NMR spectroscopy is an 
excellent for defining the transient long-range interactions and local conformations at atomic resolution on a wide timescale window (Dyson \& Wright, 2004). The solution NMR terms such as PRE, RDCs, relaxation parameters, and chemical shift perturbation are powerful in describing the conformational ensemble and protein-protein/ligand interaction for IDPs. $\alpha \mathrm{S}$ as paradigm in the structural studies of IDPs has been well characterized by these solution NMR methods (Bertoncini et al, 2005b; Cho et al, 2007; Mukrasch et al, 2007; Salmon et al, 2010; Skora et al, 2006).

In one subcategory of IDPs which includes $\alpha \mathrm{S}$, Tau et al., the proteins are able to fold and assemble into higher ultramolecular structures and are thus highly involved in the neurodegenerative diseases (Uversky, 2009; Uversky et al, 2008). According to the prevaling theory, the folding of the nascent polypeptide chain passes through the folding intermediates or partially folded states, to the native state that is located at the lower bottom positions in the funnel-shaped folding energy landscape [Figure 1.5] (Hartl et al, 2011; Hartl \& Hayer-Hartl, 2009). Due to the ruggedness of the free-energy landscape, several kinetically trapped conformations during the folding need to overcome the energy barrier to reach an energy-favored downhill path. Protein chaperones play an important role in facilitating this energy conversion and controlling the conformational correctness of folding.

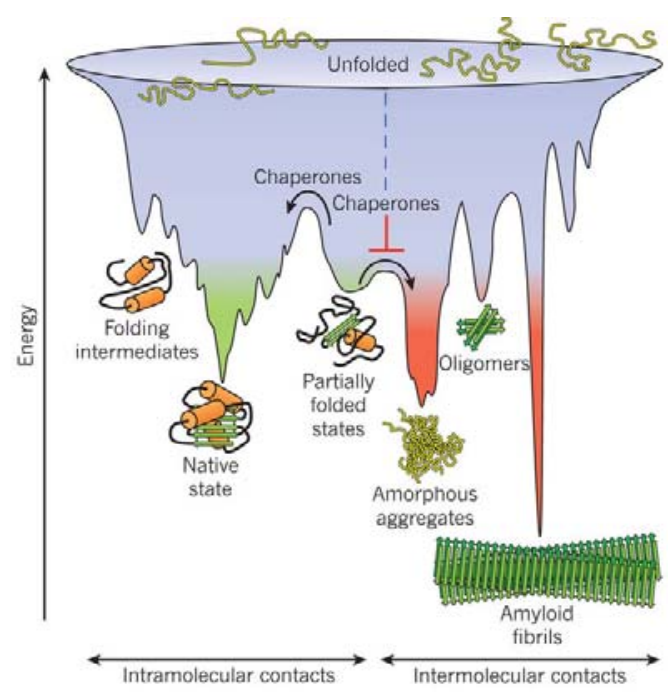

Figure 14. Schematic representation of funnel-shaped folding energy landscape. The kinetically trapped conformations as the folding intermediates present in the folding or aggregation pathway. The figure is adapted from (Hartl et al, 2011). 
The aggregation normally occurs as a consequence of the energy surface of intramolecular folding overlaping with the surface of intermolecular aggregation in the energy funnel. As described in Figure 1.4, this is the reason that proteins might through partially folded states adopt the aggregation pathway to misfold to the amorphous or the ordered aggregates. For $\alpha \mathrm{S}$, Tau, and $\mathrm{A} \beta$, due to their hydrophobic regions highly exposing to outer environment, they are readily able to achieve the intermolecular association with neigbouring proteins in cellular (Fink, 2006). Therefore, they preferentially fold though the downhill routine of intermolecular aggregation in the energy funnel to form well-ordered amyloid fibrils. This fibrillar aggregation typically occurs in a nucleation-dependent manner for the oligomerization. As protein chaperones are able to inhibit the misfolding, the study of interactions of the chaperones (i.e. HSPs) with $\alpha \mathrm{S}$ and their role in modulating $\alpha \mathrm{S}$ cellular toxicities is an active field in the research of Synucleinpathies (Dedmon et al, 2005a; Falsone et al, 2009; Huang et al, 2006; Liu et al, 2009; Lo Bianco et al, 2008; Putcha et al, 2010; Roodveldt et al, 2009). At the same time, the aggregation of $\alpha \mathrm{S}$ has been developed as the promising therapeutic target for PD treatments (Brundin \& Olsson, 2011; Madine \& Middleton, 2009).

\subsection{Major challenges}

The major research interests and challenges are summarized in the following section.

(1) Assess structures of the $\alpha \mathrm{S}$ oligomers at atomic resolution and characterize the interaction of proteins/ligands with $\alpha \mathrm{S}$ intermediates.

(2) Clarify the toxic species in the scenario of $\alpha \mathrm{S}$ aggregation, monomers, oligomers, fibrils or establish alternative hypothesis.

(3) Define the functional relevance of posttranslational modification, particularly the dominant phosphorylation at Ser129 of $\alpha \mathrm{S}$.

(4) Identify the protein-protein interactions involved in $\alpha \mathrm{S}$ pathologies.

(5) Investigate the structural basis for the cell-to-cell prion-like transmission.

(6) Detect the origin and functional relevance of polymorphism of $\alpha \mathrm{S}$ aggregates 
(7) Search for the interplay between different PD genes.

The issue (1)-(4) will be discussed in this dissertation, as based on our experimental data. 



\section{Materials and Methods}

\subsection{Materials}

\subsubsection{Facility}

Table 2.1 Facility used in sample reparation, NMR measurements and biophysical studies Facility

Specifications

Atomic force microscope

Balances

Centrifuge

Concentrators

Dialysis

Dynamic light scattering

Electron microscope

Fluorescence

Filter (syringe)

Gel Electrophoresis

Incubator

Lyophilization

NMR spectrometers

UV-Vis Photometer

\subsubsection{Chemicals}

Application

Bacterial phage for RDC Fibril dissolving

Phosphorylation

Other chemicals
Asylum Research MFP-3D ${ }^{\mathrm{TM}}$ Stand Alone Atomic Force Microscope Sartorius B 3100 S \& AC 210 S

Eppendorf Centrifuge 5415D

Eppendorf Centrifuge 5804

Beckman TL-100 with TLA-100.3 rotor

Amicon Microcon, Centricon, Centriplus/ Amicon Ultra, Ultracel

Thermo Scientific Slide-A-Lyzer Dialysis Cassettes

Wyatt DynaPro Titan

Philips CM12

Varian Cary Eclipse Spectrophotometer

Millipore $0.2 \mu \mathrm{m}$ syringe filter

BioRad Power Pac 300

Thermo Scientific, Heraeus, B6 and B20

Braun Biotech Christ Alpha 2-4

Bruker Avance $400 \mathrm{MHz}$, TXI probe,

Bruker AvanceIII $600 \mathrm{MHz}$, QXI probe

Bruker Avance $600 \mathrm{MHz}$, cryoprobe

Oxford Avance 700 III MHz, TXI probe

Bruker Avance $700 \mathrm{MHz}$, cryoprobe

Bruker Avance III $800 \mathrm{MHz}, 1.7 \mathrm{~mm}$ and 5.0mmcryoprobe,

Bruker Avance $900 \mathrm{MHz}$, cryoprobe

Hewlett-Packard 8453

Table 2.2 Chemicals used in experiments.

Chemicals

Pf1, Asla, Riga, Latvia

GuSCN, Sigma-Aldrich, Deisenhofen, Germany

PLK1-3, Cell Signaling Technologies and Invitrogen, USA

CKI, New England Biolabs, USA,

Thioflavin T, Sigma, Steinheim, Germany

DTT, Gerbu, Gaiberg, Germany

Bench Mark protein ladder, Invitrogen, Karlsruhe, Germany

SDS, Serva, Heidelberg, German

Acetonitrile, APS, ATP, HEPES, TEMED, Roth, Karlsruhe, Germany

Formic acid, Glycine, Hydrochloric acid, Manganese chloride 
dihydrate, Sodium chloride, Sodium dihydrogen phosphate, Sodium hydroxide, TFA, Tris, Merck, Darmstadt, Germany

\subsubsection{Software}

Table 2.3 Software

Programe

Topspin 2.1

NMRPipe/NMRDraw

Sparky 3

MARS(graphic interface)

PALES

Pymol

MATHEMATICA

ORIGIN 7.5

Excel:mac
Source

Bruker Biospin, Karlsruhe, Germany spin.niddk.nih.gov/NMRPipe (Delaglio et al. 1995)

www.cgl.ucsf.edu/home/sparky (Goddard and Kneller)

http://www3.mpibpc.mpg.de/groups/zweckstetter/ links/software mars.htm (Jung 2004, Narayanan 2010)

http://www3.mpibpc.mpg.de/groups/zweckstetter/_links/software_pales.htm (Zweckstetter and Bax 2000, Zweckstetter 2008)

DeLano Scientific LLC, Palo Alto, CA, USA

Wolfram Research, Champaign, IL, USA

OriginLab Corporation, Northampton, MA, USA

Microsoft, USA

\subsection{Sample preparation}

\subsubsection{Protein expression and purification}

Sample expression and purification of $w t-\alpha \mathrm{S},{ }^{15} \mathrm{~N} w t-\alpha \mathrm{S},{ }^{15} \mathrm{~N}-\mathrm{E} 46 \mathrm{~K} \alpha \mathrm{S},{ }^{15} \mathrm{~N}-\mathrm{A} 53 \mathrm{~T} \alpha \mathrm{S}$, $\mathrm{P} 128 \mathrm{E} / \mathrm{S} 129 \mathrm{E} \alpha \mathrm{S}, \mathrm{M} 127 \mathrm{E} / \mathrm{P} 128 \mathrm{E} / \mathrm{S} 129 \mathrm{E} \alpha \mathrm{S}$ were performed by Dr. Stefan Becker, Karin Giller, Sebastian Wolff in Dept. NMR-based Structural Biology, Max Planck Institute for Biophysical Chemistry in Göttingen. Wild-type $\alpha \mathrm{S}$ and its mutants were expressed in E. coli in M9 medium and purified as described (Bertoncini et al, 2005b; Hoyer et al, 2002). The samples of ${ }^{15} \mathrm{~N}-\mathrm{A} 30 \mathrm{P} \alpha \mathrm{S},{ }^{15} \mathrm{~N}-\mathrm{E} 46 \mathrm{~K} \alpha \mathrm{S},{ }^{15} \mathrm{~N}-\mathrm{A} 53 \mathrm{~T} \alpha \mathrm{S}$ for realtime NMR assays were prepared by Prof. Dr. Hialal Lashuel's group.

Rab1b-GDP, Rab1b-GppNHp, Rab3a-GDP, Rab3a-GppNHp, Rab8a-GDP, Rab8a-GppNHp, Rab8a(6-176)-GDP, Rab8a(6-176)-GppNHp, ${ }^{15} \mathrm{~N}-\mathrm{Rab} 1 b-G D P,{ }^{15} \mathrm{~N}-$ Rab8a-GDP, ${ }^{15} \mathrm{~N}-R a b 1 b(1-175)-G D P,{ }^{13} \mathrm{C},{ }^{15} \mathrm{~N}-R a b 1 b(1-175)-G D P,{ }^{13} \mathrm{C},{ }^{15} \mathrm{~N}-\mathrm{Rab} 8 \mathrm{a}-$ GDP were prepared by the group of Dr. Amyelt Itzen, Dept. Physical Biochemistry, Max Planck Institute of Molecular Physiology in Dortmund.

\subsubsection{Synthesis of Rab8a C-terminal peptide}

The C-terminal Rab-peptide of Rab8a comprising residues $\mathrm{Asp}^{176}-\mathrm{Arg}^{205}$ was synthesized by Kerstin Overkamp (Dept. NMR-based Structural Biology, Max Planck 
Institute for Biophysical Chemistry in Göttingen) by using standard solid-phase fluorenylmethoxycarbonyl chemistry. The peptide was purified by semipreparative reverse-phase HPLC, and the purity ( $>95 \%$ ) was analyzed by MS.

\subsubsection{Preparation of the phosphorylated Ser129 $\alpha$-Synuclein}

$\alpha$-Synuclein was dissolved in the phosphorylation buffer containing $\mathrm{Mg}^{2+}, \mathrm{ATP}$ and the phosphorylation was stated by the addition of Polo-like Kinase 3 (PLK3). The solution was incubated at $30{ }^{\circ} \mathrm{C}$ for 2.5 hours to reach full phosphorylation at the Ser129. Trace amount of the phosphorylation product was loaded into MS to identify the single phosphorylation which was indicated by the mass change of $80 \mathrm{Da}$ compared with wt$\alpha \mathrm{S}$ [Figure 2.1 (A) and (B)]. The phosphorylated $\alpha \mathrm{S}$ was purified by semipreparative reverse-phase HPLC coupled with the online monitors of UV-Vis and MS. The purified protein was lyophilized and dissolved into the aggregation buffer. The single phosphorylation was further confirmed by 1D NMR spectrum [Figure 2.1 (C)]. The steps of HPLC/MS were implemented by Gerhard Wolf (Dept. NMR-based Structural Biology, Max Planck Institute for Biophysical Chemistry in Göttingen).

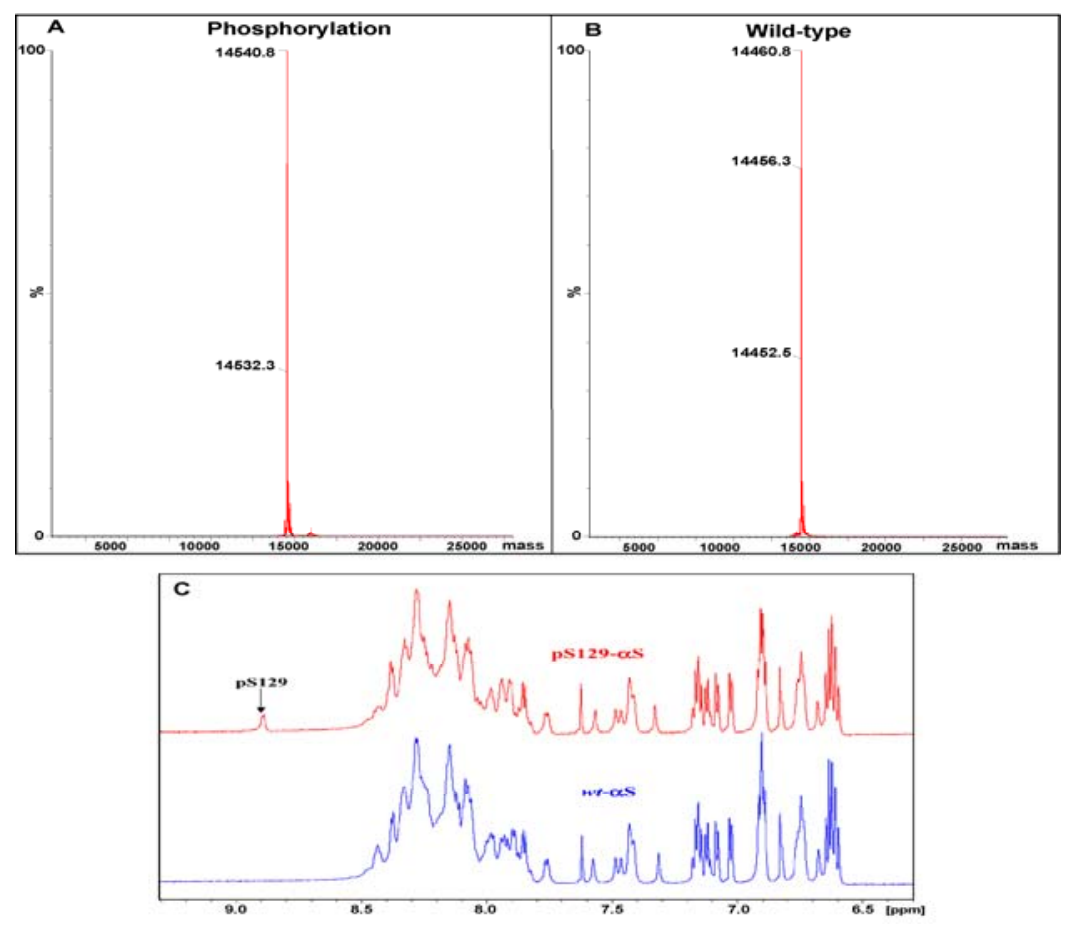

Figure 2.1 Characterization of Ser129-phosphorylation of $\alpha \mathrm{S}$. Mass spectrum of the phosphorylated $\alpha \mathrm{S}(14540.8 \mathrm{kDa})(\mathrm{A})$ and $w t-\alpha \mathrm{S}(\mathrm{B})$ as a reference $(14460.8 \mathrm{kDa}) .(\mathrm{C}) 1 \mathrm{D}{ }^{1} \mathrm{H}$ NMR spectra comparison demonstrates the single peak of the phosphorylated Ser129 occured after PLK3 phosphorylation. 


\subsection{Methods}

\subsubsection{Phosphorylation assays}

\section{PLKs phosphorylation}

For real-time NMR to measure the enzymatic kinetics, samples contained $\sim 0.1$ $\mathrm{mm} 15 \mathrm{~N}$-labeled $\alpha \mathrm{S}$ in $20 \mathrm{~mm}$ HEPES, $10 \mathrm{~mm} \mathrm{MgCl} 2,2 \mathrm{~mm}$ dithiothreitol, and 1.09 mm ATP, pH 6.9. The real-time assay was started by the addition of kinase into the sample, with a protein/kinase ratio of 100:0.5. The temperature was set to $303 \mathrm{~K}$ to allow for efficient phosphorylation. To reduce the impact of signal broadening due to amide proton exchange, the temperature was lowered to $288 \mathrm{~K}$ during the measurement of ${ }^{1} \mathrm{H},{ }^{15} \mathrm{~N}$ heteronuclear single quantum coherence (HSQC) spectra. In the case of PLK3, the phosphorylation reaction proceeded very rapidly at $303 \mathrm{~K}$. Therefore, we also followed the kinetics of phosphorylation of $\alpha \mathrm{S}$ by PLK3 at 293 and $288 \mathrm{~K}$ using 0.075 $\mathrm{mm} \alpha \mathrm{S}$ (protein $/$ kinase $=100: 0.5 \mu \mathrm{g})$.

\section{CKI}

The kinase was incubated at $37^{\circ} \mathrm{C}$ for 5 minutes before mixing it with the protein $(225$ $\mu \mathrm{M}$ ) in CK1 Reaction Buffer (New England Biolabs), pH 7.4. The reaction was carried out in the presence of $1.09 \mathrm{~mm}$ ATP and 1200 units of CK1/145 $\mu \mathrm{g}$ of $\alpha \mathrm{S}$. Afterwards, the phosphorylation was started and monitored by the real-time NMR method at $15^{\circ} \mathrm{C}$.

\subsubsection{Aggregation assays}

Aggregation assays for $\alpha \mathrm{S}$ performed in this thesis follow the steps: 1) concentration calibration (UV-Vis, NMR); 2) solution processing: filtering with $0.22 \mu \mathrm{m}$ membrane to remove of large particles and ultracentrifuge with $60 \mathrm{~K} \mathrm{rpm}$ to remove the oligomeric species; 3) aggregation with constant stirring at specified temperature. Thioflavin-T (ThT) as specific dye was used to monitor the fibril-formation kinetics. Dynamic light scattering (DLS) was used to monitor the oligomerization process. Electron microscopy (EM) and Atomic force microscopy (AFM) were selectively used to morphologically characterize the $\alpha \mathrm{S}$ aggregates. 
Three buffer systems are used in this thesis. (1) HEPES buffer: HEPES 50mM, $\mathrm{NaCl} 100 \mathrm{mM}, \mathrm{pH} 7.4$, used in the assays: aggregation in the presence of the Rab proteins, aggregation of the phosphorylated $\alpha \mathrm{S}$ and the phosphorylation mimics. (2) Phosphate buffer: Phosphate $50 \mathrm{mM}, \mathrm{NaCl} 100 \mathrm{mM}, \mathrm{pH} 7.4$, used in the generation of the compounds-stablized oligomers. (3) Tris buffer: Tris $20 \mathrm{mM}, \mathrm{NaCl} 100 \mathrm{mM}, \mathrm{pH} 7.4$, used in the producing the fibrils of the pathological $\alpha \mathrm{S}$ mutants. $0.1 \% \mathrm{NaNa}_{3}$ is normally included in the buffer for sterilization.

Standard temperature for $\alpha \mathrm{S}$ aggregation is at $37^{\circ} \mathrm{C}$. For the aggregation assays in the presence of Rab proteins, the temperature was set at $21^{\circ} \mathrm{C}$ taking into account the stability issue of Rab proteins. And the co-aggregating factors i.e. ligands, proteins should be added before the filtering step.

\subsubsection{NMR spectroscopy}

\subsubsection{Backbone assignment}

\section{Backbone assignment methods}

Backbone assignment, determing the nucleus that the resonance originates from, is the primary work to analyze the structure of macromolecules like proteins and nuclear acids by NMR spectroscopy (Wüthrich, 1986), particularly after the advent of multidimensional spectroscopy (Bax \& Grzesiek, 1993; Oschkinat et al, 1988). Protein backbone assignment is achieved by transferring of coherence along the network of scalar coupled spins ${ }^{1} \mathrm{HN},{ }^{15} \mathrm{~N},{ }^{13} \mathrm{C}_{\alpha}\left({ }^{13} \mathrm{C}_{\beta}\right)$, and ${ }^{13} \mathrm{CO}$ of the ${ }^{13} \mathrm{C},{ }^{15} \mathrm{~N}$ isotropic labeling protein in 3D NMR experiments [Figure 2.2] (Sattler et al, 1999). INEPT (Insensitive Nuclei Enhanced by Polarization Transfer) is a fundamental block responsible for the coherence transfer based on the scalar coupling $(\mathrm{J})$ specific to the different coupled spins. INEPT is designated to transfer nuclear spin polarization from spins with large Boltzmann population differences to nuclear spins of interest with low Boltzmann population differences (Morris \& Freeman, 1979). To obtain maximal sensitivity, the proton with largest gyromagnetic ratio $(\gamma)$ is firstly polarized by the excitation of pulse in most of the cases and then the polarization was transferred by INEPT to the heteronuclei that this proton attaches to i.e. ${ }^{15} \mathrm{NH},{ }^{13} \mathrm{C}_{\alpha},{ }^{13} \mathrm{C}_{\beta}$. The following phase is performing the mixing and evolution, by which the coherence is transferred along the 
pathway targeting the specified sequential connectivity and chemical shifts of the indirection dimensions were labeled by encoding the modulation of Larmor frequencies of each nuclear in spin system to the magnetization strength. The final phase is transferring the magnetization back to the proton which would be detected by recording the FID.

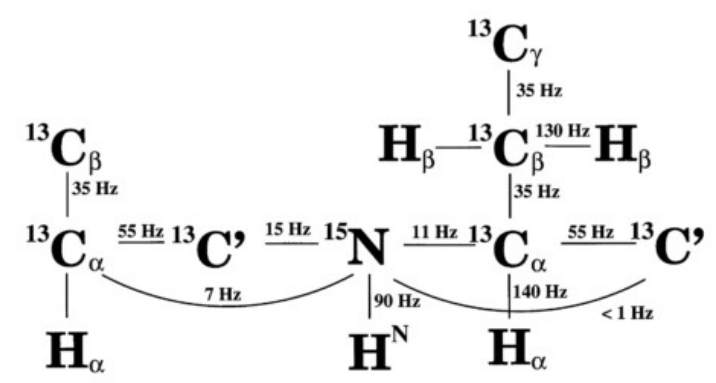

Figure 2.2 Network of the scalar coupling along protein backbone. Spin system of the peptide backbone and the size of the ${ }^{1} \mathrm{~J}$ and ${ }^{2} \mathrm{~J}$ coupling constants that are used for magnetization transfer in ${ }^{13} \mathrm{C}$-, ${ }^{15} \mathrm{~N}$-labelled proteins. Figure is adapted from (Sattler et al, 1999).

There are two manners, "out and back" and "one way", commonly used for the INEPT transfer pathway for constructing 3D experiments. The "out and back" experiments such as $\mathrm{HNCO}, \mathrm{HNCA}, \mathrm{HNCACB}$ are in one category experiments that magnetization transfer was started and ended at same protons. The "one way" is that the transfer is started from one proton and ended at another proton, for instance the CBCAcoNH is started from $\mathrm{H}_{\alpha} / \mathrm{H}_{\beta}$ and ended at amide HN. The time set for the INEPT delay is taken into account the compromise between sufficient coherence transfer based on $\mathrm{J}$ coupling and the diphase caused by transverse relaxation $\left(\mathrm{R}_{2}\right)$ during the longer delay time as described in the following formula:

$$
\Delta_{o p t}=\frac{1}{2 \pi J} \cdot a \cdot \tan \left(\pi J T_{2}\right),
$$

where $\mathrm{J}$ is the coupling constant and $T_{2}$ is the transverse relaxation time. Therefore, the relaxation features should be estimated prior to start recording of 3D experiments. One-one echo experiment designed based on the rotation frame is a straightforward and ready way to estimate the $T_{2}$, global rotational correlation time $\left(\tau_{c}\right)$ and thereby the molecular weight of the protein that assessing situation of the oligomerization (Sklenar \& Bax, 1987). At the same time, 1D NMR spectrum can also 
be used as an evidence to judge if protein is folded after sample preparation procedure. However, the proteins with larger molecular weights $(\geq 20 \mathrm{kDa})$ exhibit worse the spectra qualities with respect to the sensitivity and resolution, which are the results of strong nuclear relaxation and singal overlap because of the large number of residues. A major trend in the development of NMR spectroscopy applied in structural biology is extending the detection limits posed by the large molecular weight of proteins. Transverse relaxation optimized spectroscopy (TROSY) is one approach that improves the spectra qualities by select the component with the mutual cancellation of dipoledipole couplings (DD) and chemical shift anisotropy (CSA), where the transverse relaxation of the large proteins are dominated by these two mechanisms (Pervushin et al, 1997). TROSY as the alternative to heteronuclear correlation spectroscopy (HSQC) can be incorporated into the well-established 3D or higher dimension experiments (Salzmann et al, 1998) and thus successfully enables NMR assignment and the following structural analysis for the large proteins.

Following the acquisition of 3D experiments required for establishing the sequential connectivity [Table 2.3.1], the backbone assignments will be implemented by the steps of spin system recognition and sequential connectivity searching. Herein, the assignments were performed by MARS, a robust automatic backbone assignment program (Jung \& Zweckstetter, 2004a; Jung \& Zweckstetter, 2004b). The manual peakpicking and prediction of secondary chemical shifts based on the primary sequence are required prior to running MARS. The specified assignments, the residual dipolar couplings (RDCs), dihedral angles and existed crystal structure can be optionally contained in the input data. The sequential connectivity of the local fragment and globular backbone is established by linking the inter- and intra-residue signals and mapped onto the protein sequence, which the secondary structure propensity is taken into account. The assignment is finally output by combining the local and globular assignment. 
Table 2.3.1 3D experiments used for backbone assignments of the Rab proteins

\begin{tabular}{|l|l|l|}
\hline Experiments & Bruker pulse code & Parameters ${ }^{\perp}$ \\
\hline HNCA & trhncagp3d2 & $\begin{array}{l}50 \times 24 \times 1024 \mathrm{pts} ; \mathrm{ns}=16 ; 600 \mathrm{MHz} \\
{ }^{*} 50 \times 25 \times 1024 \mathrm{pts} ; \mathrm{ns}=24 ; 800 \mathrm{MHz}\end{array}$ \\
\hline HNCACB & trhncacbgp3d & $\begin{array}{l}68 \times 21 \times 1024 \mathrm{pts} ; \mathrm{ns}=32 ; 700 \mathrm{MHz} \\
{ }^{*} 61 \times 26 \times 1024 \mathrm{pts} ; \mathrm{ns}=48 ; 800 \mathrm{MHz}\end{array}$ \\
\hline $\mathrm{CBCA}(\mathrm{CO}) \mathrm{NH}$ & trcbcaconhgp3d & $\begin{array}{l}64 \times 20 \times 1024 \mathrm{pts} ; \mathrm{ns}=36 ; 700 \mathrm{MHz} \\
{ }^{*} 64 \times 23 \times 1024 \mathrm{pts} ; \mathrm{ns}=32 ; 800 \mathrm{MHz}\end{array}$ \\
\hline $\mathrm{HNCO}$ & trhncogp3d & $50 \times 33 \times 1024 \mathrm{pts} ; \mathrm{ns}=32 ; 700 \mathrm{MHz}$ \\
\hline $\mathrm{HN}(\mathrm{CA}) \mathrm{CO}$ & trhncacogp3d & $65 \times 21 \times 1024 \mathrm{pts} ; \mathrm{ns}=32 ; 700 \mathrm{MHz}$ \\
\hline
\end{tabular}

${ }^{\perp}$ Specification for spectrometer parameters: number of complex points (pts) in $t_{1}, t_{2}$ and $t_{3}$ time domain; number of scanes (ns); field strength for ${ }^{1} \mathrm{H}$

\section{Structural analysis based on chemical shifts}

After the backbone assignment is obtained, we are able to perform the structural analysis for protein based on the chemical shifts. Firstly, the secondary chemical shifts of carbon $\left(\mathrm{C}_{\alpha}, \mathrm{C}_{\beta}, \mathrm{CO}\right)$ are highly indicative of the structural propensity of secondary structure. The secondary chemical shifts were determined by the difference between the experimental chemical shifts $\left(\delta_{\exp }\right)$ and the random coil chemical shifts $\left(\delta_{\text {coil }}\right)$ of the residue (Schwarzinger et al, 2001; Schwarzinger et al, 2000):

$$
\Delta \delta=\delta \exp -\delta_{\text {coil }}
$$

To correct the referencing errors, the difference between the secondary chemical shifts of $\mathrm{C}_{\alpha}$ and $\mathrm{C}_{\beta}$ has been applied (Wang et al, 2005):

$$
\Delta \delta_{\alpha}-\Delta \delta_{\beta}
$$

Secondly, residual dipolar couplings (RDCs) can be measured by adapting the protein into the weak alignment mediums i.e. the liquid crystal and bacterial phage where the dipolar coupling between two nuclear dipoles is partially retained due to the motion of molecule is restricted in the medium (Prestegard et al, 2004). For instance, the RDCs of NH vector can be measured by comparing the difference of scalar coupling of $\mathrm{NH}$ between the aligned sample and isotropic sample (in free solution). To this aim, the IPAP-HSQC (Ottiger et al, 1998) and interleaved TROSY/HSQC (Kontaxis et al, 2000) are two common approaches to measure the J-couplings. 
Herein, the RDCs for ${ }^{15} \mathrm{~N}-\mathrm{Rab} 1 \mathrm{~b}-\mathrm{GDP}$ were obtained from the interleaved TROSY/HSQC spectra:

$$
{ }^{1} D_{N H}=2 \cdot\left(v_{N}^{H S Q C}-v_{N}^{T R O S Y}\right)
$$

At the same time, the RDC values were back-calculated from the crystal structure of Rab1b (PDB code: 3NKV) using singular value decomposition (SVD) by the program PALES (Zweckstetter, 2008). A comparison of the experimental and the calculated RDCs was used to evaluate the difference between solution conformation and crystal structure. The solution conformation matches the crystal structure well if the Q-factor of fitting is below 0.2 .

\subsubsection{HSQC-based study of protein-protein/ligand interactions}

NMR signals of backbone amides provide excellent information to probe proteinprotein/ligand interaction with the description of interaction interface and binding affinity according to perturbation of chemical shift and line-width analysis (Craik \& Wilce, 1997; Gao et al, 2004). In practice, ${ }^{1} \mathrm{H},{ }^{15} \mathrm{~N}-\mathrm{HSQC}$ spectra have been recorded for the ${ }^{15} \mathrm{~N}$-labeled protein in the absence and presence of the interaction partners that are normally unlabeled proteins or ligands. The chemical shifts perturbations are calculated as:

$$
\Delta \delta_{H N}=\sqrt{\left(\Delta \delta_{N} / 5\right)^{2}+\left(\Delta \delta_{H}\right)^{2}}
$$

The HSQC spectra of ${ }^{15} \mathrm{~N}$-labeled sample are recorded in the presence of the binding partners at different molar ratios to the sample. In this case, the chemical shift perturbations are increased as a function of concentration of the binding partners, which the dissociation constant $\mathrm{K}_{\mathrm{d}}$ can be estimated by fitting to a single-site binding model (Cavanagh et al, 2007):

$$
\Delta \delta=\Delta \delta_{\max } \cdot\left([L]_{T}+[P]_{T}+K_{d}-\left\{\left([L]_{T}+[P]_{T}+K_{d}\right)^{2}-4[L]_{T} \bullet[P]_{T}\right\}^{1 / 2}\right) /\left(2[P]_{T}\right)
$$

where the $\Delta \delta_{\max }$ is the maximal chemical shift perturbation value at saturation, $[\mathrm{P}]_{\mathrm{T}}$ and $[\mathrm{L}]_{\mathrm{T}}$ are the total concentration of protein and ligand. This approach is particularly advantageous to determine the $K_{d}$ for weak binding system $\left(K_{d}>10^{-6} M\right)$. The 
crystallization for such system is difficult or impossible (Cavanagh et al, 2007) and ITC used for measuring such weak binding might bear bigger uncertainty.

\subsubsection{Real-time NMR method used in the investigation of phosphorylation kinetics}

Real-time NMR methods used in studies of the enzymatic kinetics possess predominant advantages than other common methods like MS spectrometry and immunodetection (Landrieu et al, 2006). Firstly, 2D NMR HSQC spectrum is able to unambiguously identify the residues undergoing phosphorylations and the resonances of the phosphorylated residues are located at the downfield region of the spectrum which is well-resolved with the unphosphorylated resonance. Secondly, the enzymatic resolution can be monitored at atomic resolution, which the onset time and reaction rate can be assessed for different phosphorylation sites simultaneously.

For our phosphorylation kinetics studies, ${ }^{15} \mathrm{~N}$-labelled $\alpha \mathrm{S}$ and the assignment for

${ }^{1} \mathrm{H},{ }^{15} \mathrm{~N}-\mathrm{HSQC}$ of $\alpha \mathrm{S}$ are required. The real-time assay was started immediately after the sample was mixed with the kinases and ATP. A series of HSQC spectra were recorded and quantitative analysis of the phosphorylation kinetics was implemented based on the measurement of resonance intensity. For better time resolution, the fast acquisition pulse SOFAST-HMQC was applied to investigate the fast kinetics instead of common HSQC (Schanda \& Brutscher, 2005; Schanda et al, 2005). SOFAST, "band-Selective Optimized-Flip-Angle Short-Transient”, is a technique to design the pulse sequence based on the Ernst-angle excitation:

$$
\cos \alpha_{o p t}=\exp \left(-t_{r e c} / T_{1}\right)
$$

where $\alpha_{\text {opt }}$ is the optimized flip angle of the excitation or so-called "Ernst angle", $t_{\text {rec }}$ is the time of recover delay and $T_{1}$ is the longitudinal relaxation constant. Here, the SOFAST is combining this technique with the selective ${ }^{1} \mathrm{H}$ pulses which are aiming to optimize the longitudinal relaxation.

\subsubsection{NMR approach to study H/D exchange rate of protein backbone}

Hydrogen/Deuterium exchange method has been widely applied to characterize the structures of amyloid fibrils (Carulla et al, 2010). In this dissertation, this method based on solution NMR has been used for structural analysis of $\alpha \mathrm{S}$ fibrils and Oligomers. For 
$\alpha \mathrm{S}$ fibrils, as demonstrated in Figure 2.3, the collected fibrils were adapted into $99.9 \%$ $\mathrm{D}_{2} \mathrm{O}$ solution containing $200 \mathrm{mM} \mathrm{NaCl}$ and $0.1 \%$ formic acid (pD 4.0) for two weeks. During this period, the hydrophobic core buried inside fibrils should be protected against the solvent but other fraction of protein not in the core would exposose to the solvent and thus was exchanged by deuterium. After this exchange period, the fibrils were collected again and dissolved by GuSCN into NMR observable monomers which encode the protection information. This dissolving solution contains $50 \% \mathrm{D}_{2} \mathrm{O}$ only and at $\mathrm{pH}$ 2.4. Thus the residue previously expososed to the solvent would experience a back-exchange from $99.9 \%$ deuteration to $50 \%$. The sample was transferred into NMR spectrometer immediately after the dissolving in order to start the acquisition of the HSQC spectra with the average dead time of 15 minutes and acquisition time of 25 minutes. The HSQC spectra were recorded at $5^{\circ} \mathrm{C}$ to restrict the proton exchange rate. The residue from protected or exposed regions could be distinguished by mapping the change of resonance intensity against the time course. The increasing pattern of intensity denotes this residue was in an exposure region before, whereas the previously protected region would have the flat or decreasing patterns.

The working principle of $\mathrm{H} / \mathrm{D}$ exchange for $\alpha \mathrm{S}$ oligomers is similar. However, the distinct nature of the oligomers requires the modifications for most of steps. Firstly, for the exchange, $\mathrm{D}_{2} \mathrm{O}$ has been directly added to the solution containing the oligomers and the time for the exchange was within 10 minutes since the hydrophobic protection in oligomers is expected to be wearker than in the fibril core. Secondly, the GuSCN was then directly added into the solution and adjust the $\mathrm{pH}$ down to 2.4 , which the solution volume was thus expanded.

In addition, to shorten the acquisition time for better time resolution, we used HSQC with band-selective excitation Short-Transient (BEST-HSQC) (Lescop et al, 2010). The BEST-HSQC replaces all proton hard pulses by band selective excitation pulses to only excite the amide protons of protein and remain the other type protons and water signals not to be directly excited. The spin-lattice relaxation of a ${ }^{1} \mathrm{H}$ spin depends on the initial spin-state of the surrounding ${ }^{1} \mathrm{H}$ spins. The excited amide protons can exchange polarization with surrounding non-excited aliphatic protons and water protons though NOE or spin diffusion effects, by which the $\mathrm{T}_{1}$ relaxation of the amide protons 
can be shortened. By this way, the recover delay can be shortened, which both repetition rate and sensitivity can be improved.

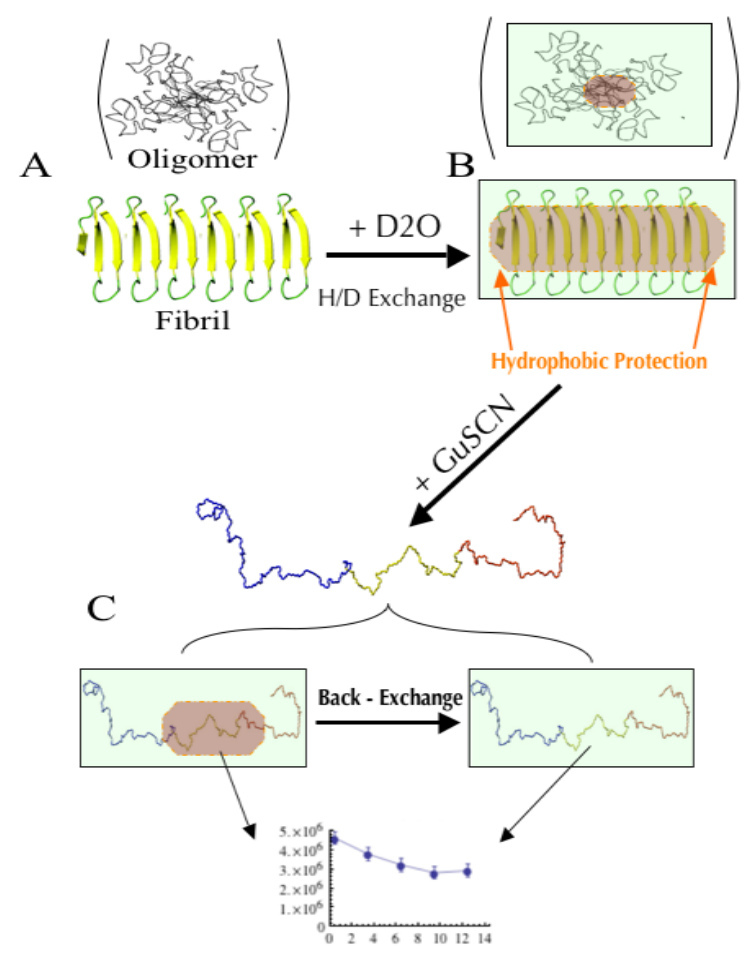

Figure 2.3 Schematic flowchart of $\mathbf{H} / \mathbf{D}$ exchange. $\alpha \mathrm{S}$ aggregates (fibrils/oligomers) (A) are adapted into the $\mathrm{D}_{2} \mathrm{O}$ buffer (B) for a specified period. The hydrophobic core region of the aggregates should be protected against the solvent exchange. After the exchange period, the aggregates are dissolved by GuSCN into the monomers encoding the protection information (C) and quickly transferred to the NMR spectrometer to start recording a series of HSQC spectra. Finally, the change of residue-specific peak intensity reflects the extent of protection/exposure.

\subsubsection{Diffusion coefficient}

NMR diffusion experiments detect the translational diffusion of molecules in solution by using pulsed field gradients (Johnson, 1999). The spatial enconding for the molecules is firstly implemented by the pair of pulsed field gradients (bipolar gradients) coupled with one spin-echo pulse. Then there will be a diffusion period that allows the molecules to move away from the original encoding position.During this period, the motional rate of the molecules depends on the diffusion coefficient. After this diffusion time, the position of the molecule can be decoded by the second pair of pulsed field 
gradients with same net strength but opposite direction. The gradient strength applied in the spectrum is normally varied from $25 \%$ to $95 \%$. The diffusion coefficient of the molecule is estimated by fitting the signal intensities as the function of varying gradient strength according to:

$$
I=I_{0} e^{-D \gamma^{2} g^{2} \delta^{2}(\Delta-\delta / 3)}
$$

where I is the observed intensity, $\mathrm{I}_{0}$ the reference intensity, $\mathrm{D}$ the diffusion coefficient, $\gamma$ the gyromagnetic ratio of the observed nucleus, $\mathrm{g}$ the gradient strength, $\delta$ the length of gradient and $\Delta$ the diffusion time. For $\alpha \mathrm{S}$, the peaks located with the range of 0.5-2.0 ppm were integrated for the fitting. The diffusion coefficient is defined by StokesEinstein equation:

$$
D=\frac{k T}{6 \pi \eta R_{h}}
$$

where $\mathrm{K}$ is the Boltzman constant, $\mathrm{T}$ the temperature, $\eta$ the viscosity of the solution and $\mathrm{R}_{\mathrm{h}}$ hydrodynamic radius of the molecules. Thus, we can measure the hydrodynamic radius of the protein by referring to the internal reference molecule, dioxane, with known $\mathrm{R}_{\mathrm{h}}(2.12 \AA)$. In our measurements, we used the pulsed field gradient spin-echo (PGSE) sequence with the incorporation of WATERGATE (W5) element which significantly suppresses the water signal (Zheng et al, 2008).

\subsubsection{Biophysical methods}

\subsubsection{Fluorescence spectroscopy}

Fluorescence measurements with specified excitation and emission wavelength enable us to detect specific information. Thioflavin-T (ThT) is a dye specific to the structure of crossing $\beta$-sheets and thus widely used to characterize kinetics of the fibril formation process (LeVine, 1999). The excitation wavelength is $446 \mathrm{~nm}$ and the emission wavelength is 460 to $600 \mathrm{~nm}$, where the maximum emission is at $482 \mathrm{~nm}$ for amyloid fibrils. For the fluorescence measurement, $5 \mu \mathrm{L}$ in-time aggregation solution is mixed into $5 \mu \mathrm{M} \times 2 \mathrm{~mL}$ ThT solution. 


\subsubsection{UV-Vis Spectroscopy}

UV absorption at $280 \mathrm{~nm}$ is used to determine protein concentration according to BeerLambert law:

$$
A_{280}=\varepsilon_{280} \cdot c \cdot d
$$

$\mathrm{A}_{280}$ : the absorption at $280 \mathrm{~nm}$

$\varepsilon_{280}$ : The molar extinction parameters at $280 \mathrm{~nm}$ calculated by the TRP, TYR amino acid content in the protein sequence.

c: protein concentration $(\mathrm{M})$

$\mathrm{d}$ : path length of light $(\mathrm{cm})$

\subsubsection{Dynamic light scattering}

Dynamic light scattering (DLS) is an approach to measure the particle size, namely hydrodynamic radius, in solution based on the evaluation of their translational diffusion constants of Brownian motion, which is also known as Photon Correlation Spectroscopy. DLS was performed by Wyatt Dynamo Titan with temperature control. Sample that would be used for DLS measurement should be filtered by $0.22 \mu \mathrm{M}$ membrane at beginning. If solution conains large fibrils, it should be centrifuged at $10 \mathrm{k} \mathrm{rpm}$ for at least 15 mins and only supernatant can be used for DLS measurement. Then, the sample is transferred into DLS cuvette and $18 \mu \mathrm{L}$ as a minimal volume are required. The DLS measurement was implemented at $25^{\circ} \mathrm{C}$. The laser power is set at $15 \%$ and can be adjusted according to the size of oligomeric species formed during aggregation. Acquisition time for each scan is 20 seconds and each measurement contains 20 scans. The measurement is normally repeated for $3 \sim 5$ times.

The hydrodynamic radius calculated based on the light scattering data are containing the contribution of hydration. To keep control, the diffusion measurements (DOSY) performed by NMR spectroscopy are an alternation and the gyrations measured by small angle X-ray scattering are a complementary approach to further investigate the configuration of particles. 


\subsubsection{Electron microscopy}

Transmission electron microscopy (TEM) for characterization of $\alpha \mathrm{S}$ aggregates, the oligomers and fibrils, were performed by Dr. Dietmar Riedel and Gudrun Heim in Max Planck Institute for Biophysical Chemistry. Aggregated protein samples were deposited on Formvar-coated 200 mesh copper grids (Electron Microscopy Sciences) at a concentration of $25 \mu \mathrm{M}$. For more concentrated sample, the dilution by buffer is required for better resolution. Grids were washed with two drops of water and stained with two drops of freshly prepared $0.75 \%(\mathrm{w} / \mathrm{v})$ uranyl acetate (Electron microscopy sciences). Specimens were inspected on a Philip CIME 12 electron microscope, operated at $80 \mathrm{kV}$. Digitized photographs were recorded with a slow scan CCD camera.

\subsubsection{Atomic force microscopy}

Atomic force microscopy (AFM) is an alternation approach to TEM for characterizing the $\alpha \mathrm{S}$ aggregates. The set-up is installed in Dept. NanoBiophotonics, Max Planck Institute for Biophysical Chemistry.

The aliquots of $3 \sim 5 \mu \mathrm{L}$ from aggregation system were deposited on freshly cleaved mica. After drying in air for $1 \mathrm{hr}$, unbound sample and buffer were removed by 3 times washing with $30 \mu \mathrm{L}$ distilled water. The samples were imaged using an Asylum MFP3D AFM machine, with a resonant frequency of about $100 \mathrm{kHz}$, a scan frequency of $1 \mathrm{~Hz}$ using silicone nitride tips. 


\section{Results}

\subsection{Phosphorylation of alpha-Synuclein}

Phosphorylation at Ser129 of $\alpha \mathrm{S}$ is one of major posttranslational modifications. Approximately $90 \%$ of $\alpha \mathrm{S}$ is phosphorylated at this site in pathological inclusion-Lewy body, a hallmark of PD (Fujiwara et al, 2002). Ser87 at NAC region of $\alpha$ S can also be phosphorylated by kinases, inhibiting the fibrillization of $\alpha \mathrm{S}$ (Paleologou et al). However, the functional role of $\alpha \mathrm{S}$ phosphorylation has still not been defined. Therefore, based on atomic resolution provided by NMR spectroscopy, we investigated the residue-specific kinetics of phosphorylation at both Ser87 and Ser129 of $\alpha \mathrm{S}$ as mediated by different kinases.

We first investigated the effects of 3 disease mutations of $\alpha \mathrm{S}, \mathrm{A} 30 \mathrm{P}, \mathrm{E} 46 \mathrm{~K}$ and A53T on phosphorylation kinetics of Ser87 and Ser129. Two kinases, CKI and PLK2 were respectively used in this assay to phosphorylate $\alpha \mathrm{S}$ mutants. Furthermore, PLKs phosphorylate $\alpha \mathrm{S}$ selectively at Ser129 but not Ser87 in CNS as well in vitro (Inglis et al, 2009; Mbefo et al). This offers us an opportunity to study the phosphorylation specifically at Ser129. Thus, the kinetics of $\alpha \mathrm{S}$ phosphorylation mediated by PLK1, 2, and 3 were investigated. Finally, we studied whether phosphorylation at Ser129 of $\alpha \mathrm{S}$ can affect its aggregation. We generated Ser129-phosphorylation (pS129) $\alpha \mathrm{S}$ and two phosphorylation mimics, P128E/S129E $\alpha \mathrm{S}$ and M127E/P128E/S129E $\alpha \mathrm{S}$. Aggregation assays for pS129 $\alpha \mathrm{S}$ and its two mimics were implemented in vitro. These results extended our understanding the structural mechanism and aggregation behaviors involved in $\alpha \mathrm{S}$ phosphorylation.

\subsubsection{Phosphorylation of disease-associated $\alpha$-Synuclein mutations}

Three familial mutations in gene coding of $\alpha \mathrm{S}(S N C A)$ were linked to the autosomal dominant inherited form of PD: A53T (Golbe et al, 1990; Polymeropoulos et al, 1997; Puschmann et al, 2009), A30P (Kruger et al, 1998) and E46K (Zarranz et al, 2004). 
Increasing evidence suggests that these point mutations can alter the monomeric conformation, fibril formation, and membrane binding and thereby inducing differently physiological and pathological behaviors (Bisaglia et al, 2009; Bodner et al, 2010; Bussell \& Eliezer, 2001). Therefore, our study of the effects of these three mutations on the phosphorylation enables us to depict the interplay between the familial mutations and the major posttranslational modifications.

\subsubsection{Kinetics of $\alpha$-Synuclein phosphorylation by PLK2}

Heteronuclear NMR spectroscopy on ${ }^{15} \mathrm{~N}$-labeled protein modified by a kinase allows the identification of the phosphorylation sites, measures the level of integration, and yields kinetic data for the enzymatic modification of the individual sites (Landrieu et al, 2006). Although the reaction mixture contains enzyme, ATP and $\alpha \mathrm{S}$, filtering through the ${ }^{15} \mathrm{~N}$ label allows monitoring of the kinase activity in the NMR tube without any further sample purification. To obtain single-residue resolution and identify all potential phosphorylation sites, the enzymatic reaction was followed by two-dimensional ${ }^{1} \mathrm{H}-{ }^{15} \mathrm{~N}$ heteronuclear correlation spectra. For $w t-\alpha \mathrm{S}$ and its 3 familial mutants, the resonances were sharp and showed a limited dispersion of chemical shifts, reflecting a high degree of backbone mobility [Fig. 3.1.1 A]. Phosphorylated serine residue was readily detected because phosphorylation shifts their amide proton resonance downfield of $8.8 \mathrm{ppm}$, to an empty region of the ${ }^{1} \mathrm{H}_{-}{ }^{15} \mathrm{~N}$ HSQC spectrum.

With increasing incubation time, the intensity of the NMR signal of phosphorylated Ser129 increased with the concomitant decrease of unphosphorylated Ser129. In agreement with data from cell assays (Mbefo et al, 2010), only Ser129 was phosphorylated, highlighting the specificity of PLK2 for Ser129 phosphorylation. Quantitative analysis of the phosphorylation kinetics of $w t-\alpha \mathrm{S}$ and the 3 mutants was implemented based on the measurement of resonance intensity. After one day, 40-50\% of the $w t$ protein, and the three $\alpha \mathrm{S}$ mutants were phosphorylated at Ser129, and the reaction reached saturation in the following 10 hours [Fig.3.1.1 B]. A comparison of the kinetics of phosphorylation revealed that $w t-\alpha \mathrm{S}$ was phosphorylated faster than all three genetic variants under controlled in vitro conditions using the same kinase and protein concentrations. Although the saturation levels are only slightly different, after four 
hours of incubation the degree of Ser129 phosphorylation of $w t \alpha \mathrm{S}$ was larger by a factor of 1.5 when compared to the genetic mutants.

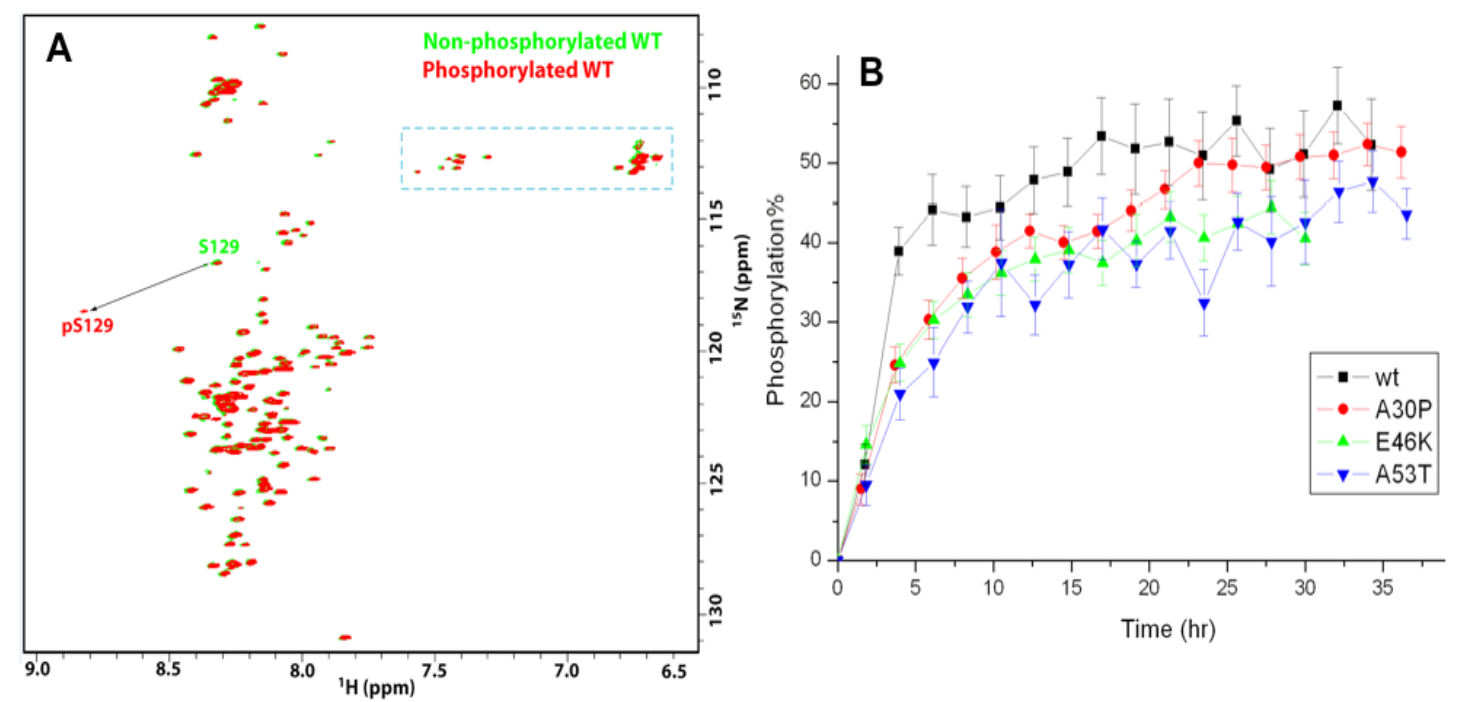

Figure 3.1.1. Kinetics of mutant $\alpha \mathrm{S}$ phosphorylation by PLK2. (A) Comparison of two-dimensional ${ }^{1} \mathrm{H}-{ }^{15} \mathrm{~N}$ HSQC spectra of unphosphorylated $w t$ (green) and PLK2-phosphorylated WT $\alpha \mathrm{S}$ (red). A dashed rectangle marks glutamine and asparagine side chain resonances. Note that PLK2 phosphorylates $\alpha \mathrm{S}$ only at Ser129. (B) Kinetics of in vitro phosphorylation of Ser129 by PLK2 in wt (black), A30P (red), E46K (green) and A53T (blue) $\alpha \mathrm{S}$. The reactions were monitored by real-time NMR spectroscopy. Error bars are based on the signal/noise ratio observed in the NMR spectra.

\subsubsection{Kinetics of $\alpha$-Synuclein phosphorylation by CK1}

Real-time NMR spectroscopy was also used to follow the kinetics of phosphorylation of $w t-\alpha \mathrm{S}$ and its 3 familial mutants by CK1. The NMR signals appearing in the downfield region of the HSQC spectrum were assigned to phosphorylated Ser87 and phosphorylated Ser129. These two downfield signals were seen in the spectra of $w t-\alpha \mathrm{S}$ and the 3 mutants, indicating that CK1 is capable of phosphorylating $w t-\alpha \mathrm{S}$ as well as the three genetic variants at both sites.

During the phosphorylation reaction, the resonance intensities of phosphorylated Ser87 and Ser129 increased, and their unphosphorylated counterparts decreased [Fig.3.1.2 A]. The rate of phosphorylation at Ser87 was identical for all three genetic mutants and $w t \alpha$-syn [Fig.3.1.2 B]. At the same time, a comparison of the kinetic profiles of phosphorylation at Ser129 $\alpha$-syn revealed a slightly higher rate of 
phosphorylation in the case of $w t-\alpha \mathrm{S}$ [Fig.3.1.2 C]. It should be noted that the highly similar phosphorylation kinetics of all four proteins at Ser87 served as a ruler and proved that the conditions of the phosphorylation reaction were identical.
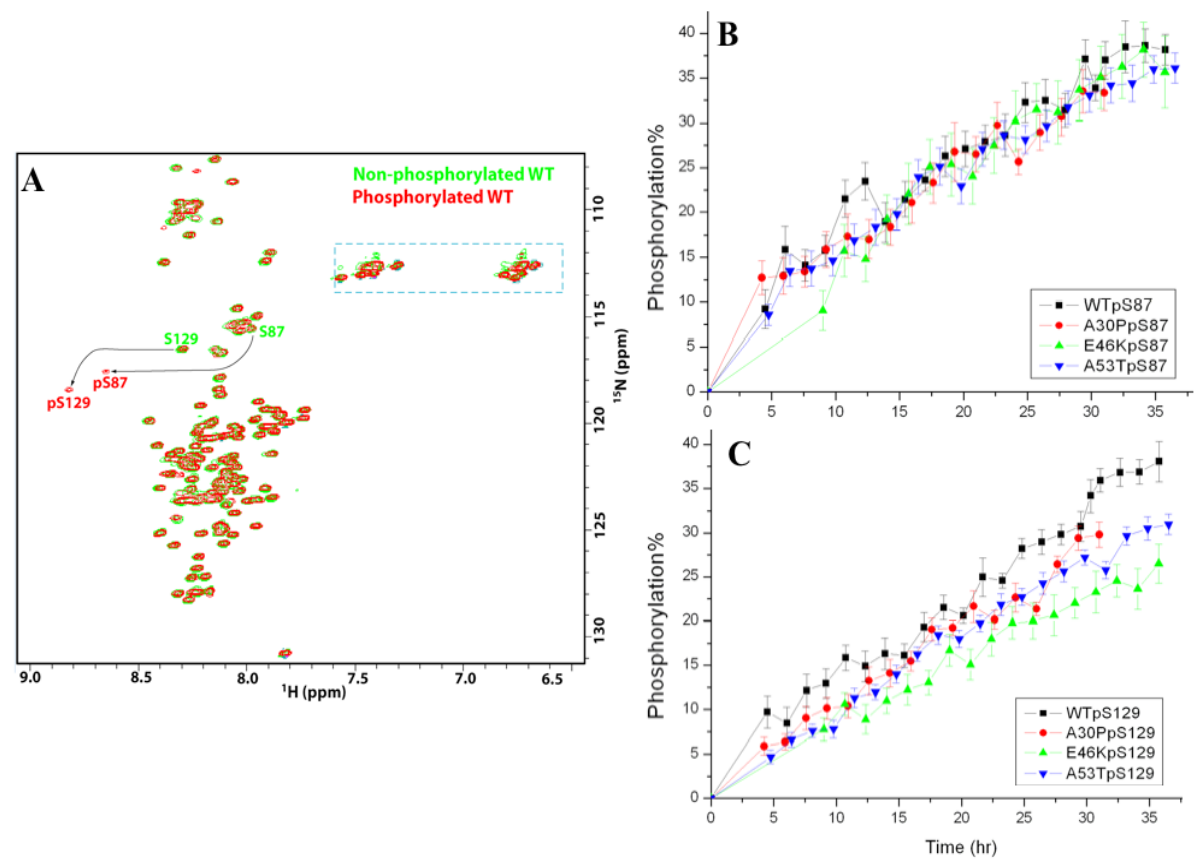

Fig. 3.1.2. Kinetics of phosphorylation of mutant $\alpha \mathrm{S}$ by CK1. (A) Comparison of two-dimensional ${ }^{1} \mathrm{H}-$ ${ }^{15} \mathrm{~N}$ HSQC spectra of unphosphorylated $w t$ (green) and CKI-phosphorylated $\alpha \mathrm{S}$ (red). Both Ser129 and Ser87 are phosphorylated. Kinetics of in vitro phosphorylation of Ser87 (B) and Ser129 (C) by CKI in $w t$ (black), A30P (red), E46K (green) and A53T (blue) $\alpha \mathrm{S}$. The reactions were monitored by real-time NMR spectroscopy.

\subsubsection{Phosphorylation of $\alpha$-Synuclein at Ser129}

he study of the postmortem human brain shows that a significantly increased level of phosphorylation occurs at Ser129 of $\alpha \mathrm{S}$, from less than $5 \%$ of the population in healthy human brains to $89 \%$ in the brains of PD patients (Visanji et al; Waxman \& Giasson, 2011). Recently, extensive research has been devoted to several issues regarding Ser129 phosphorylation, such as its impact on aggregation (Fujiwara et al, 2002), relevance to neuronal toxicity (Gorbatyuk et al, 2008; Sancenon et al, 2012), membrane binding (Kuwahara et al, 2012; Visanji et al, 2011) et al. In term of $\alpha \mathrm{S}$ primary structure, Ser129 is located at the highly flexible and acidic C-terminal which plays an important role in ligand binding, enzymatic recognition, the maintainence of protein conformation and 
modulating aggregation. Previous studies have indicated that the transient long range interactions existing between C-terminus and N-terminus were modified by the Ser129 phosphorylation and thereby the aggregation profile of $\alpha \mathrm{S}$ was potentially affected (Paleologou et al, 2008).

As described in last section 3.1.1, PLK2 is suggested as a major kinase responsible for specific phosphorylation of Ser129 in neurons (Inglis et al, 2009). Together with the in vivo methods, we investigated three closely related members of the human Polo-like kinases (PLKs) family (PLK1, PLK2, and PLK3), to observe their enzymatic performances on phosphorylating $\alpha \mathrm{S}$ in different cell cultures and catalysis kinetics in vitro (Mbefo et al, 2010). Furthermore, to assess impacts of the Ser129 phosphorylation on $\alpha \mathrm{S}$ aggregation, we generated phosphorylated Ser129 of $\alpha \mathrm{S}$ (pS129) for aggregation assay. Finally, two mimics of Ser129 phosphorylation of $\alpha \mathrm{S}$, $\mathrm{P} 128 \mathrm{E} / \mathrm{S} 129 \mathrm{E}$ and M127E/P128E/S129E, were studied in terms of their aggregation propensities.

\subsubsection{Kinetics comparison of different kinases (PLK1, PLK2, PLK3)}

In collaboration with Dr. Hialal Lashuel, we identified that PLK1-3 phosphorylated both $\alpha \mathrm{S}$ monomers and fibrils at Ser129 in the in-vitro assays, but PLK4 didn't phosphorylate both forms of $\alpha \mathrm{S}$ (Mbefo et al, 2010). Lashuel's lab also observed that PLK2 and PLK3 phosphorylated $\alpha$ S efficiently in both HEK293T cells and HeLa cells and these two kinases partially co-localized with pS129 in primary hippocampal neurons and cortical brain areas of $\alpha \mathrm{S}$ transgenic mice (Mbefo et al, 2010). These results suggest that PLKs play important role in the phosphorylation of $\alpha \mathrm{S}$ Ser129. Therefore, we investigated the enzymatic performance of PLKs on the Ser129 phosphorylation of $\alpha \mathrm{S}$

The real-time NMR assay was applied to monitor the PLK1-3-catalyzed phosphorylation reaction by measuring yield kinetics and identifying all potential phosphorylation sites in the cases of PLK1 and PLK3. Taking advantages of the singleresidue resolution realized by the real-time NMR method, amide proton resonance of pS129 was unambiguously shown in ${ }^{15} \mathrm{~N}-\alpha \mathrm{S}$ HSQC spectrum in the case of PLK3catalyzed reaction. Importantly, no additional NMR signals of phosphorylated residues appeared, indicating that PLK3 exclusively phosphorylated $\alpha \mathrm{S}$ at Ser129. 


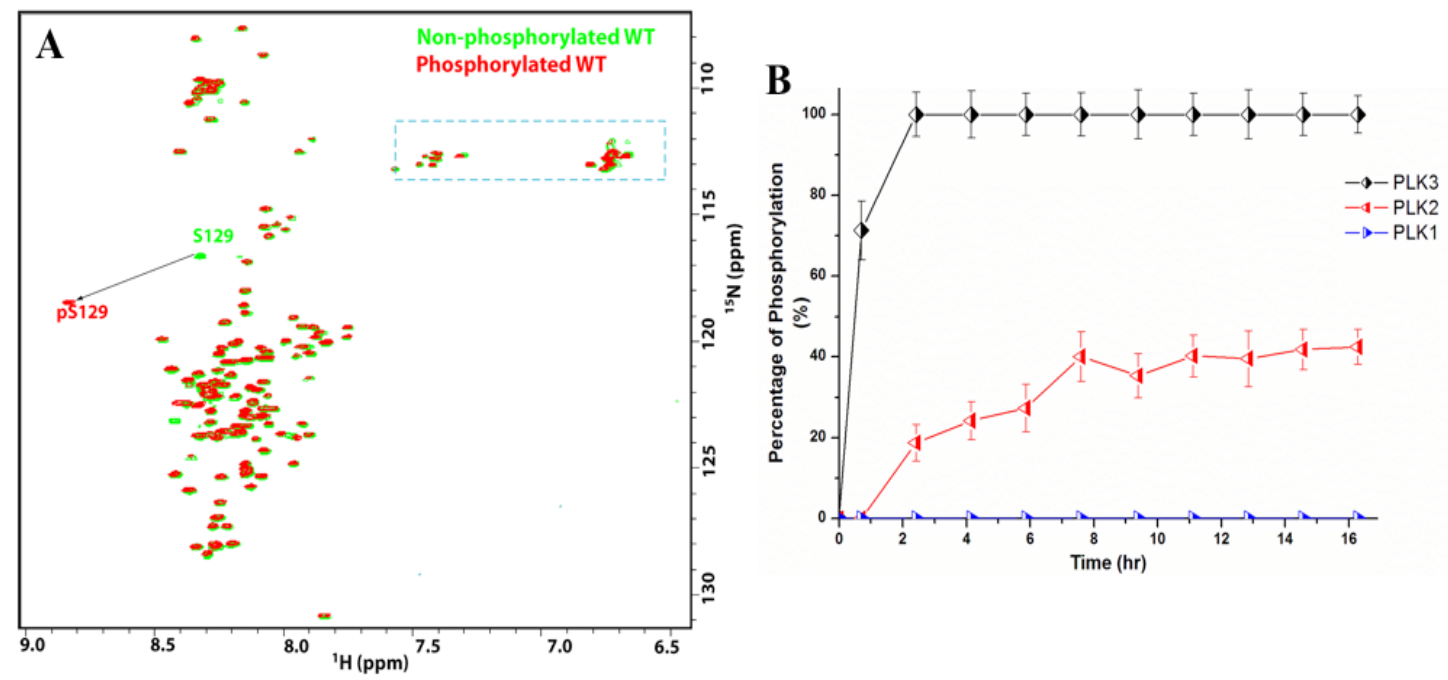

Figure 3.1.3. Phosphorylation of synucleins by PLK1-3. (A) Comparison of two-dimensional ${ }^{1} \mathrm{H}-{ }^{15} \mathrm{~N}$ HSQC spectra of unphosphorylated WT (green) and $\alpha \mathrm{S}$ phosphorylated by PLK3 (red). A dashed rectanglemarks glutamine and asparagine side chain resonances. (B) Kinetics of in vitro phosphorylation of Ser129 in $\alpha \mathrm{S}$ by PLK3 (black), PLK2 (red), and PLK1 (blue) as monitored by real-time NMR spectroscopy. NMR samples contained $\sim 0.1 \mathrm{~mm}{ }^{15} \mathrm{~N}$-labeled $\alpha \mathrm{S}$ in $200 \mathrm{~mm}$ HEPES, $10 \mathrm{~mm} \mathrm{MgCl2,} 2$ $\mathrm{mm}$ dithiothreitol, and $1.09 \mathrm{~mm} \mathrm{ATP,} \mathrm{pH}$ 6.9. The real-time assay was started by the addition of kinase into the NMR sample using a protein/kinase ratio of 100:0.5 mg. The error bars were determined based on the signal/noise ratio observed in the NMR spectra.

In the case of PLK3, the peak of phosphorylated Ser129 was already clearly observed already after 45 minutes [Figure 3.1.3 A]. With increasing incubation time, the intensity of the NMR signal of phosphorylated Ser129 increased. Quantitative analysis of the increase of the NMR signal of phosphorylated Ser-129 indicated that PLK3 fully phosphorylates $\alpha \mathrm{S}$ within 2.5 hours under the conditions of the assay [Figure 3.1.3 B]. In the case of PLK1, no phosphorylation of $\alpha \mathrm{S}$ could be detected during the time course of the experiment. The significantly reduced PLK1 phosphorylation of $\alpha \mathrm{S}$ in the NMR experiments in comparison to the in vitro kinase assay, is most likely due to the fact that the NMR phosphorylation experiments were performed at a lower temperature (15 ${ }^{\circ} \mathrm{C}$ versus $30{ }^{\circ} \mathrm{C}$ for in vitro kinase assays) to reduce the impact of signal broadening due to amide proton exchange. As previously described, real-time spectroscopy of $\alpha \mathrm{S}$ phosphorylation by PLK2 showed that PLK2 also showed phosphorylates $\alpha \mathrm{S}$ at Ser129 
only. However, the kinetics of the enzymatic reaction is significantly slower for PLK2 than for PLK3, and $\alpha \mathrm{S}$ was not fully phosphorylated at the end of the assay.

In contrast to PLK1 and PLK2, our real-time assay showed that PLK3 possessed a remarkable efficiency in phosphorylating $\alpha \mathrm{S}$. However, the fast kinetics of PLK3catalyzed phosphorylation largely limited the detailed information that can be accessed by a standard HSQC experiment at the initial stage. Consequently, we employed bandSelective Optimized-Flip-Angle Short-Transient (SOFAST) HMQC to circumvent this time restriction by shortening the time required for recording one spectrum.

The SOFAST-HMQC experiment generates the resonance signal of amide proton correlation with shorter time intervals of recovery delay between each scan than the standard HSQC spectrum. In particular, $\mathrm{T}_{1}$ relaxation optimization and Ernst angle are collectively integrated into the pulse scheme of this experiment to achieve a markedly reduction in inter-scan delay. Sensitivity and resolution are key factors for practical considerations when handling NMR experiments. To reach maximal timeshortening whilst retaining the necessary sensitivity and resolution for measurement of phosphorylation kinetics, we further narrowed spectra width in indirect dimension to reduce the time spent on a large number of incrementing points. The recycle delay was finally set at 0.3 seconds. Extraordinarily, the peak of phosphorylated Ser129 is wellresolved in the spectrum [Figure 3.1.3.A], and thereby favored all the strategies adopted above. Moreover, according to theory of enzymatic reaction, temperature is an important factor that affects reaction kinetics. By lowering the experimental temperature, we were able to slow down the reaction rate and capture more kinetic information under the given spectroscopic conditions.

Finally, we measured the kinetics of PLK3-mediated Ser129 phosphorylation by optimized SOFAST-HMQC with time resolution of 17 mins at temperature of $288 \mathrm{~K}$ and 293K. Kinetic profiles of Ser129 phosphorylation by PLK3 were mapped by measuring resonance intensity of pS129 within beginning 200 mins. Under both experimental temperatures, a distinguishable peak of the phosphorylated Ser129 already appeared after 20 min which contained the dead time and time for recording first spectrum. The peak intensity increased rapidly with increasing reaction time and approximately $90 \%$ phosphorylation was reached at 70 mins under both temperatures. After $\sim 2$ hours, the 
kinetics under both temperatures had already achieved saturation, suggesting that the reaction finished and $\alpha \mathrm{S}$ was fully phosphorylated at the Ser129 by PLK3.

Quantitative comparison between $288 \mathrm{~K}$ and $293 \mathrm{~K}$ shows that the kinetic difference is indistinguishable, indicating a temperature-independence for kinetics within this temperature range. Moreover, the kinetic curves measured by SOFASTHMQC spectra under these two temperatures are highly similar to the kinetics measured by standard HSQC spectra under temperature of 303K [Figure 3.1.3 B], at which $70 \%$ $\alpha \mathrm{S}$ has been phosphorylated at 50mins and a complete phosphorylation was reached within 2.5 hours.

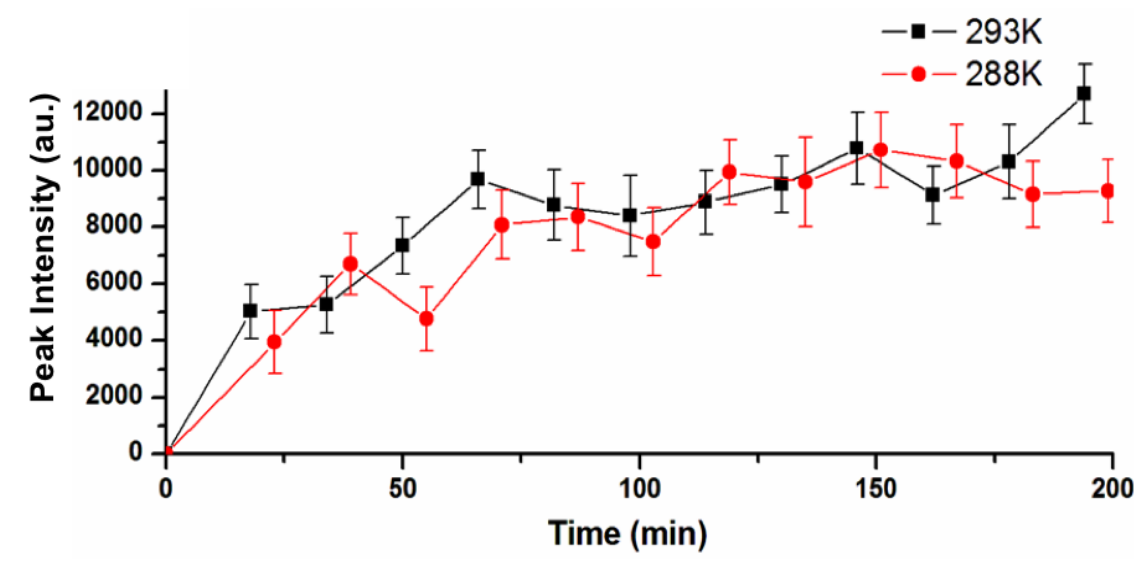

Figure 3.1.4. Phosphorylation of $\alpha S$ by PLK3 at the lower temperatures by SOFAST-HMQC. Kinetics of phosphorylation of Ser-129 of $\alpha$ S by PLK3 under temperature of 293K (black) and 288K (red) were monitored by real-time NMR spectroscopy. NMR samples contained $\sim 0.1 \mathrm{~mm}{ }^{15} \mathrm{~N}$-labeled $\alpha \mathrm{S}$ in the phosphorylation buffer for PLKs described before. The real-time assays were started by the addition of kinase into the NMR sample using a protein/kinase ratio of 100:0.5 mg. The error bars were determined based on the signal/noise ratio observed in the NMR spectra.

In summary, real-time NMR spectroscopy revealed that PLK2 and PLK3 exclusively phosphorylated $\alpha \mathrm{S}$ at Ser129, consistent with other in-vitro and in-vivo assays (Mbefo et al, 2010). Moreover, PLK3 phosphorylated $\alpha \mathrm{S}$ with significant efficiency that full phosphorylation could be achieved within 2.5 hours under the assay condition. Based on the fast acquisition technique, we further demonstrated that PLK3 phosphorylated nearly $50 \% \alpha \mathrm{S}$ within 25 mins and its enzymatic kinetics were not strongly altered by changing reaction temperature. 


\subsubsection{Aggregations assays of phosphorylated Ser129 $\alpha$-Synuclein}

One major focus on $\alpha \mathrm{S}$ phosphorylation is to address the relationship between phosphorylation and aggregation. Hitherto, the role of phosphorylation, particularly at Ser129, in modulating aggregation and the chronological order of these two events in pathological cascades of PD have still not been clearly defined. Fujiwara et al. firstly reported that $\alpha \mathrm{S}$ was phosphorylated at Ser129 by their Mass Spectrometry assays and that this modification could promote $\alpha \mathrm{S}$ aggregation in vitro (Fujiwara et al, 2002). However, by using phosphorylation-mimicking (Ser to Asp/Glu) and-retarding (Ser to Ala) mutants to simulate the effects of phosphorylation or to block other phosphorylation sites (i.e. Ser87), Paleologou et al. investigated the interplay between Ser129 phosphorylation of $\alpha \mathrm{S}$ and its aggregation and suggested that the S129 phosphorylation blocked fibril formation (Paleologou et al, 2008). Similarly to in vitro studies, accumulating in vivo findings from different cell cultures and animal models presented diversified indications regarding the role of Ser129 phosphorylation in modulating $\alpha \mathrm{S}$ aggregation and neuronal toxicity (Azeredo da Silveira et al, 2009; Chen \& Feany, 2005; Chen et al, 2009; McFarland et al, 2009; Smith et al, 2005).

Most of studies mentioned above were performed by using the specified mutants to resemble the effects of phosphorylation. However, it should be noted that phosphorylation mimics by point mutation can not completely and accurately reproduce all aspects of native phosphorylation and the mutations at other sites are likely to introduce nonspecific side-effects. Thus, to produce accurate phosphorylation at an expected site without applying additional mutations is of critical importants in our experiment.

Our previous real-time NMR assay identified the exclusive selectivity and significant efficiency of PLK3 on phosphorylation of $\alpha \mathrm{S}$ Ser129. Therefore, we chose PLK3 as the kinase to specifically phosphorylate Ser129 in vitro. To investigate whether the phosphorylation of Ser129 modulates its aggregation, the aggregation assay has been performed based on the PLK3-mediated phosphorylation is described in Figure 3.1.5.(A). 

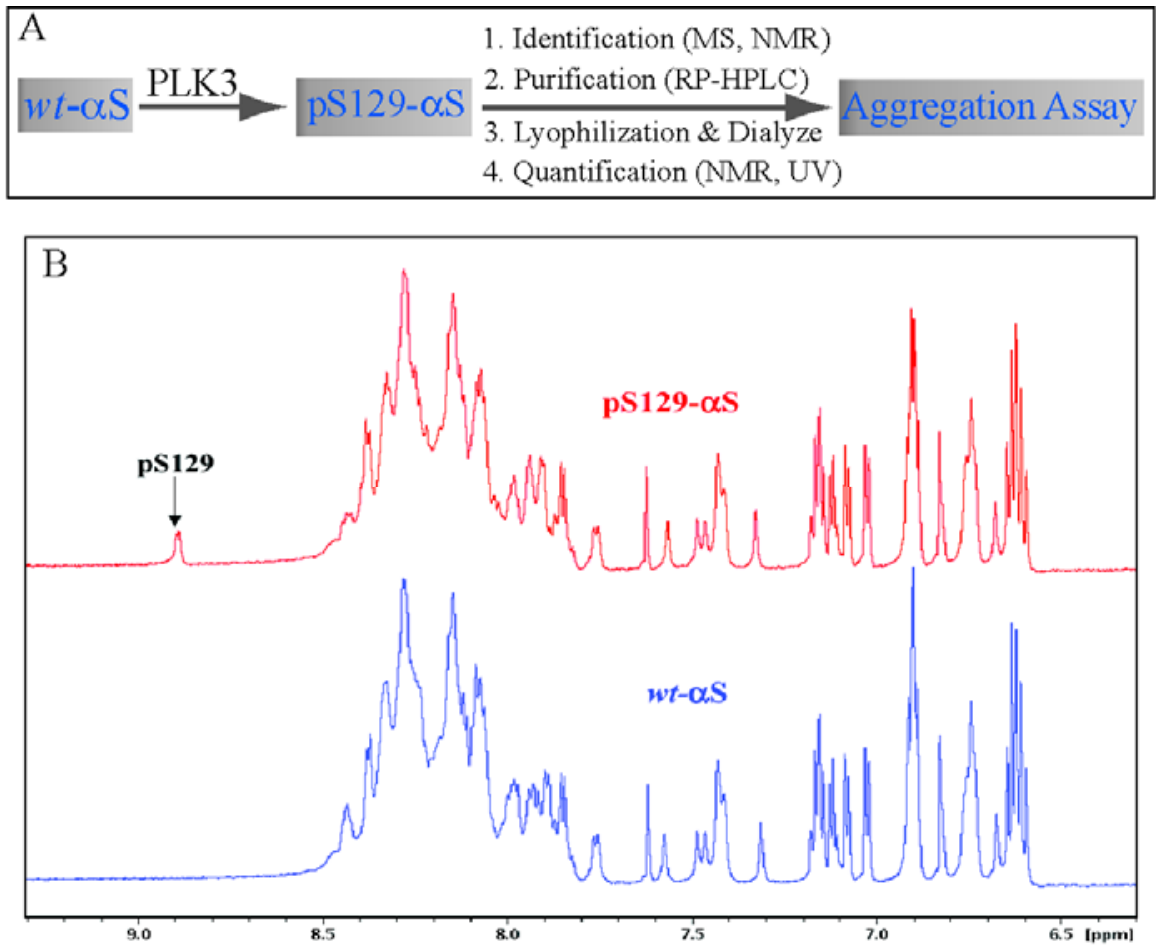

Figure3.1.5. Preparation for aggregation assay of phosphorylated-Ser129 $\boldsymbol{\alpha S}$. (A)Flow chart of the aggregation assay for $\mathrm{pS} 129-\alpha \mathrm{S}$. The consecutive steps include phosphorylation by PLK3, characterization and purification of phosphorylated sample. (B) Characterizing phosphorylation by 1D NMR spectrum. The spectrum of $w t-\alpha \mathrm{S}$ (blue) and pS129- $\alpha \mathrm{S}$ (red) are compared. In spectrum of pS129$\alpha \mathrm{S}$, single peak at $8.8 \mathrm{ppm}$ denotes the single phosphorylation at Ser129.

In the first stage, $w t-\alpha \mathrm{S}$ was adapted into the phosphorylation buffer containing $\mathrm{Mg}^{2+}$ and ATP. Then phosphorylation was started by the addition of PLK3 and incubated under $30^{\circ} \mathrm{C}$ for 2.5 hours to achieve complete phosphorylation. Secondly, trace amount of the reaction product has been checked by Mass spectrometry to identify whether there was a mass difference of 80 -Dolton in comparison to the $w t-\alpha \mathrm{S}$ sample. At the same time, the purity of the phosphorylation product was examined. Accordingly, the confirmed sample was loaded into RP-HPLC coupled with UV and MS detection to collect $\mathrm{pS} 129-\alpha \mathrm{S}$. The purified material of $\mathrm{pS} 129-\alpha \mathrm{S}$ was lyophilized to remove the solvent used in HPLC and further dialyzed against the aggregation buffer together with $w t-\alpha \mathrm{S}$ to assure that both samples dissolved in identical solution for the aggregation assay. At the end, PULCON method (Wider \& Dreier, 2006) based on NMR spectroscopy was employed to calibrate the protein concentration of pS129 $\alpha \mathrm{S}$ and $w t$ $\alpha \mathrm{S}$ aiming to produce identical concentrations for both proteins in the aggregation assay 
for both proteins. A comparison between 1D NMR spectrum of pS129 $\alpha \mathrm{S}$ and $w t-\alpha \mathrm{S}$ clearly shows one single peak at 8.8 ppm [Fig. 3.1.5(B)] which was identified as the phosphorylation of Ser129 by HSQC spectrum in the previous real-time assay.

Following the purification and concentration-calibration, the samples of pS129$\alpha \mathrm{S}$ and $w t-\alpha \mathrm{S}$ were further processed by getting rid of small particles and small oligomeric species and were consequently adapted to start the aggregation assay with a monomeric concentration of $50 \mu \mathrm{M}$. Kinetics of the fibril formation were monitored by Thioflavin T (ThT) fluorescence. Unexpectedly, within the time course of 150 hours, general kinetic trend and fibril yield reflected by ThT did not show a significant difference between $\mathrm{pS} 129-\alpha \mathrm{S}$ and $w t-\alpha \mathrm{S}$ under the assay condition [Figure 3.1.6 A]. After 40 hours of the time course, the growth rates of fibrils for both samples became much slower and no distinguishable difference between two samples was observed. This revealed that the aggregation kinetics were close to saturation and the fibril yields for these two samples were similar. In line with kinetic data, EM images revealed that fibrils of $\mathrm{pS} 129-\alpha \mathrm{S}$ [Figure3.1.6 D] and $w t-\alpha \mathrm{S}$ [Figure3.1.6 E] presented similar morphology.

Interestingly, a significant difference was observed at 24hours. Including the consideration of experimental uncertainty, the sample of pS129 $\alpha \mathrm{S}$ shows relatively stronger ThT intensity than that of $w t \alpha \mathrm{S}$ at 24 hours. This observation agrees with the structural findings that phosphorylation at Ser129 of $\alpha \mathrm{S}$ potentially modifies protein conformation by partially releasing the long-range interactions within the polypeptide chain (Paleologou et al, 2008). As an intrinsically disordered protein (IDP), the transient long range interactions between $\mathrm{N}$-terminal and $\mathrm{C}$-terminal of $\alpha \mathrm{S}$ importantly contributes to maintaining the protein conformation and inhibiting aggregation propensity(Bertoncini et al, 2005b; Dedmon et al, 2005c). Furthermore, this phenomenon was reproduced in the aggregation assay at a lower $\alpha \mathrm{S}$ concentration $(10 \mu \mathrm{M})$ shown in Figure3.1.6 (B). 

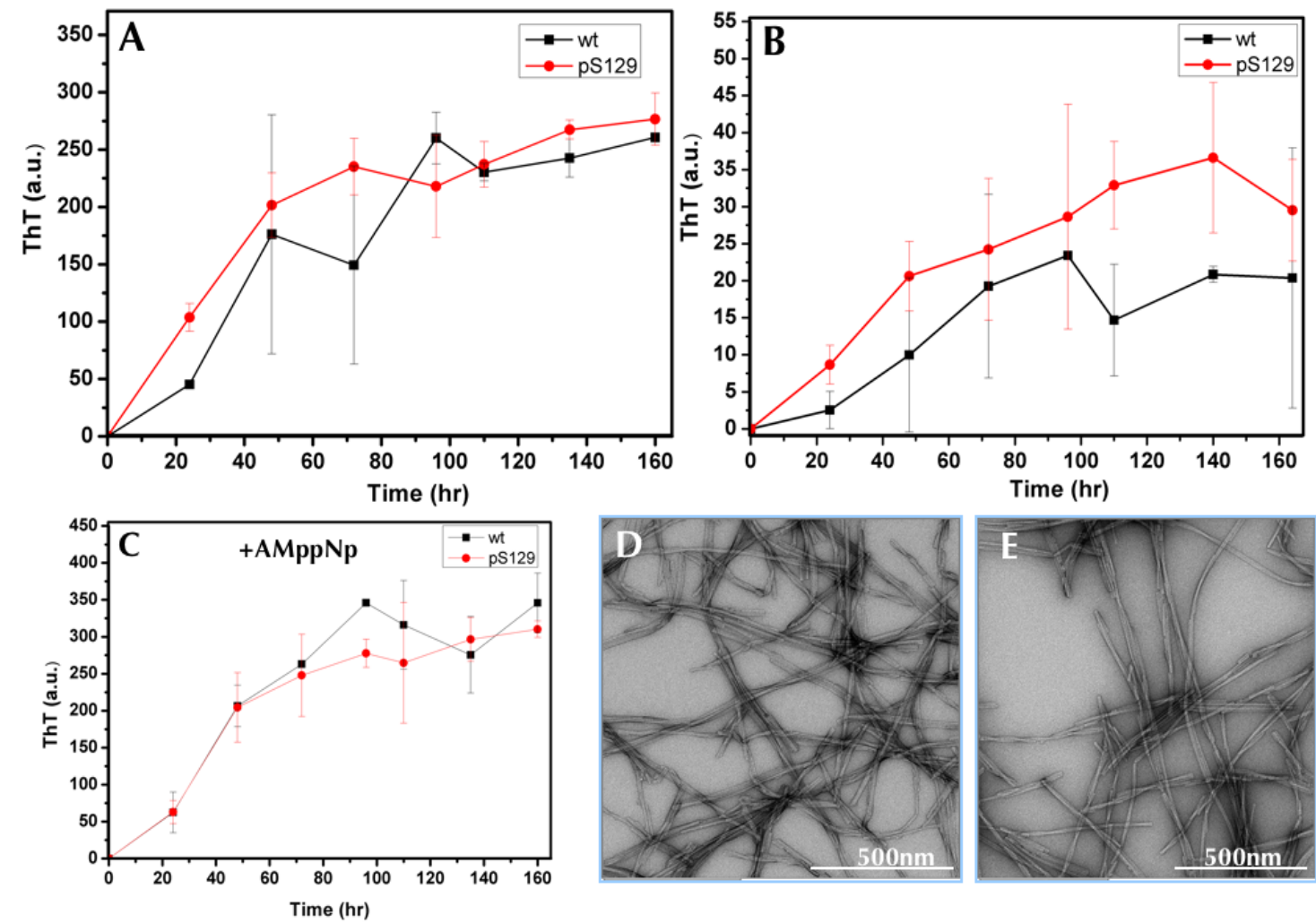

Figure 3.1.6. Aggregation assay of pS-129 $\alpha \mathrm{S}$ and $w \boldsymbol{t}-\alpha \mathrm{S}$. Fibril formation of pS129 (red) and wt- $\alpha \mathrm{S}$ (black) were followed by ThT fluorescence under the different aggregation conditions. (A) pS129- $\alpha \mathrm{S}$ and wt- $\alpha$ S started aggregation at a monomeric concentration of $50 \mu \mathrm{M}$. EM images of the final aggregation products of pS129 and $w t-\alpha \mathrm{S}$ are shown in (D) and (E) respectively. (B) Reproducing aggregation for both proteins at the lower concentration $(10 \mu \mathrm{M})$. (C) Agregation assay was performed with the presence of $\mathrm{AMppNp}(500 \mu \mathrm{M})$, a hydrolysis-resistant analogue of ATP. All error bars were estimated based on statistics of triplicate trials for each sample.

In addition, we checked whether ATP can have impact on $\alpha \mathrm{S}$ aggregation. ATP is the necessary compound for phosphorylation and is likely present during the aggregation of phosphorylation in vivo. Therefore, AMppNp, one hydrolysis-resistant analogue of ATP, has been included in the aggregation system with the amount of 10fold in excess of the proteins (AMppNp: $500 \mu \mathrm{M}$ and proteins: $50 \mu \mathrm{M}$ ). The aggregation profile in the presence of $\mathrm{AMppNp}$ is similar to the aggregation profile counterpart observed in the case of its absence. A slight increase of fibril amount during the saturation phase compared to the assay without the addition of the compound was observed in Figure 3.1.6 (C). Also, ThT intensities for the two samples at 24 hours were also similar. Hence, modifications of aggregation kinetics and fibril yield induced by 
AMppNp were not drastic, suggesting that these minute effects were non-specifically caused by this ATP analogue, but were not critical.

\subsubsection{Aggregation assays of Ser129 phosphorylation mimics of $\alpha$-Synuclein}

Although the effects of phosphorylation can not be completely reproduced by the mimics, genetic mutation of Ser to Glu or Asp still provides us opportunity and freedom to artificially modify the side-chain of the residue and simulate the steric and electrostatic properties of phosphorylation not only in vitro but also in vivo (Figure3.1.7).

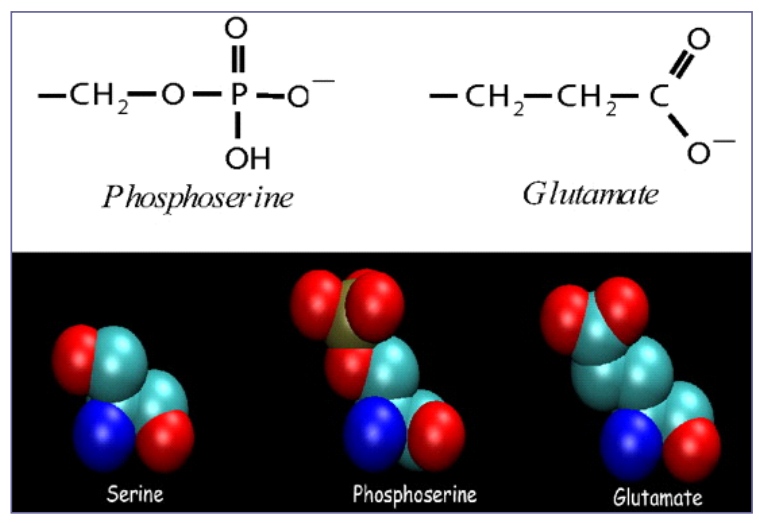

Figure3.1.7 The atomic structures of the side chains of Glu and phospho-Ser demonstrate the structural and electrostatic similarities between the two moieties. The figure is taken from the publication (Paleologou et al, 2008).

To further determine the potential roles of Ser129 phosphorylation on protein aggregation, we produced two Glutamine mutants to mimic the phosphorylation. Double mutant P128E/S129E $\alpha \mathrm{S}$ and triple mutant M127E/P128E/S129E $\alpha \mathrm{S}$ were designed aiming at multiplying the effects of phosphorylation at the neighboring region of Ser129 and hence further clarifying the issues regarding how these mutations impact on $\alpha \mathrm{S}$ aggregation.

The aggregation assay for the two mutants and $w t-\alpha \mathrm{S}$ indicated that these two mutations did not affect aggregation kinetics [Figure3.1.8]. Three samples displayed similar scales of lag phase and fibril growth rate. The final yields of fibrils were also very similar. This was also consistent with corresponding EM images, which the fibrils of the three proteins were in similar size and morphology. 


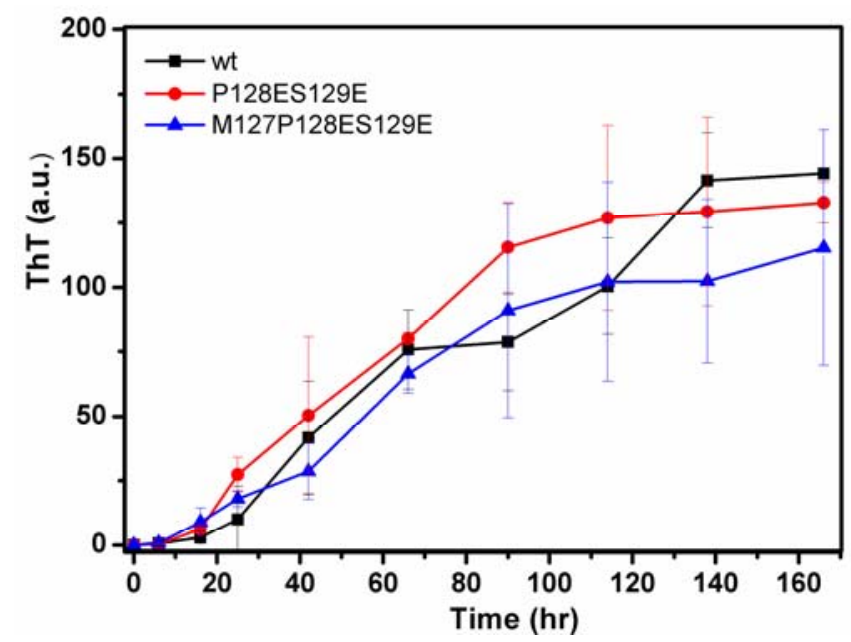

Figure3.1.8 Aggregation assay for the $\alpha \mathrm{S}$ phosphorylation mimics. The aggregation kinetics of $w t \alpha \mathrm{S}$ (black), double mutant P128E/S129E (red) and triple mutant M127E/P128E/S129E (blue) are shown.

In conclusion, we examined the aggregation propensity of $\alpha \mathrm{S}$ with Ser129 phosphorylation. Both $\alpha \mathrm{S}$ with phosphorylation at the Ser129 by kinase PLK3 in vitro and the phosphorylation mimics displayed similar aggregation kinetics of fibril formation and morphology of fibril products, suggesting that phosphorylation at this site does not significantly change the aggregation. This finding agrees with the observations that phosphorylation of Ser129 of $\alpha \mathrm{S}$ is independent of $\alpha \mathrm{S}$ aggregates formation, where the kinase (CKs and PLKs) was co-expressed with $\alpha \mathrm{S}$ in vivo (Waxman \& Giasson, 2011). 


\subsection{Interplay between alpha-Synuclein and Rab proteins}

The family of Rab GTPases belongs to Ras superfamily of small G proteins. The human Rab family consists of 70 members and most of them exert important functions in regulating vesicular trafficking and the exocytosis of living cells (Hutagalung \& Novick, 2011). In neurons, vesicle trafficking is a pivotal process responsible for neurotransmitter transport and release. The specified Rab proteins are involved in all steps of the trafficking process from the vesicle budding to final diffusion to plasmid membrane. Meanwhile, the deficit of vesicle trafficking is characterized as one phenotype of $\alpha \mathrm{S}$ neuronal toxicity (Auluck et al, 2010). In well-established yeast assay for $\alpha \mathrm{S}$ (Outeiro \& Lindquist, 2003), the overexpression of Rab1, Rab3a, and Rab8a respectively were found to rescue the $\alpha \mathrm{S}$-induced deficits occurring at multiple stages of vesicle trafficking (Cooper et al, 2006; Gitler et al, 2008).

What are the structural mechanisms underlying this rescuing effect of the Rabs? To answer this question, we performed NMR based structural analysis and correlated biophysical assays to study the interactions between $\alpha \mathrm{S}$ and Rabs. Analysis of amino acid sequences of Rab1b, Rab3a and Rab8a revealed diversity at their C-termini. Following this, we used NMR titration to study potential interactions that might exist between $\alpha \mathrm{S}$ and these Rabs at atomic resolution and found that Rab8a was capable of binding to $\alpha \mathrm{S}$. Using the peptide synthesis technique and the expression of the truncated protein, this interaction has been dissected with respect to different parts of Rab8a. To further characterize the interaction in details, we implemented the NMR assignment for Rab1b and Rab8a. Accordingly, we were able to map interaction sites on Rab8a exactly. Moreover, we also found that the binding affinity of Rab8a to $\alpha \mathrm{S}$ depending upon the different nucleotide-bound states of Rab8a.

In parallel to the studies of the interactions based on NMR structures, we also performed an aggregation assay for $\alpha \mathrm{S}$ in the presence of different Rabs. Our scope was thus extended to search for potential interactions between the Rabs and $\alpha \mathrm{S}$ aggregates and to evaluated the influence of Rab proteins on the oligomerization and fibrillization of $\alpha \mathrm{S}$. Taken together, the studies reveal the interplay between the different Rab proteins and $\alpha \mathrm{S}$ of different states. 


\subsubsection{Sequence alignment of Rab1b, Rab3a and Rab8a}

The in vivo investigation demonstrates that the overexpression of Rab1, Rab3a and Rab8a can suppress dopaminergic neuron loss caused by overexpression of human $\alpha \mathrm{S}$ in C. elegans and primary rat midbrain cultures (Gitler et al, 2008). Interestingly, in the model of C. elegans, Rab8a reduced the neurodegeneration of affected worms from $85 \%$ to $60 \%$, whereas Rab1 reduced the neurodegeneration to $80 \%$ and Rab3a reduced to $75 \%$. Thus, this suggests that 3 Rab proteins might differentiate mechanistically in their interactions with $\alpha \mathrm{S}$.

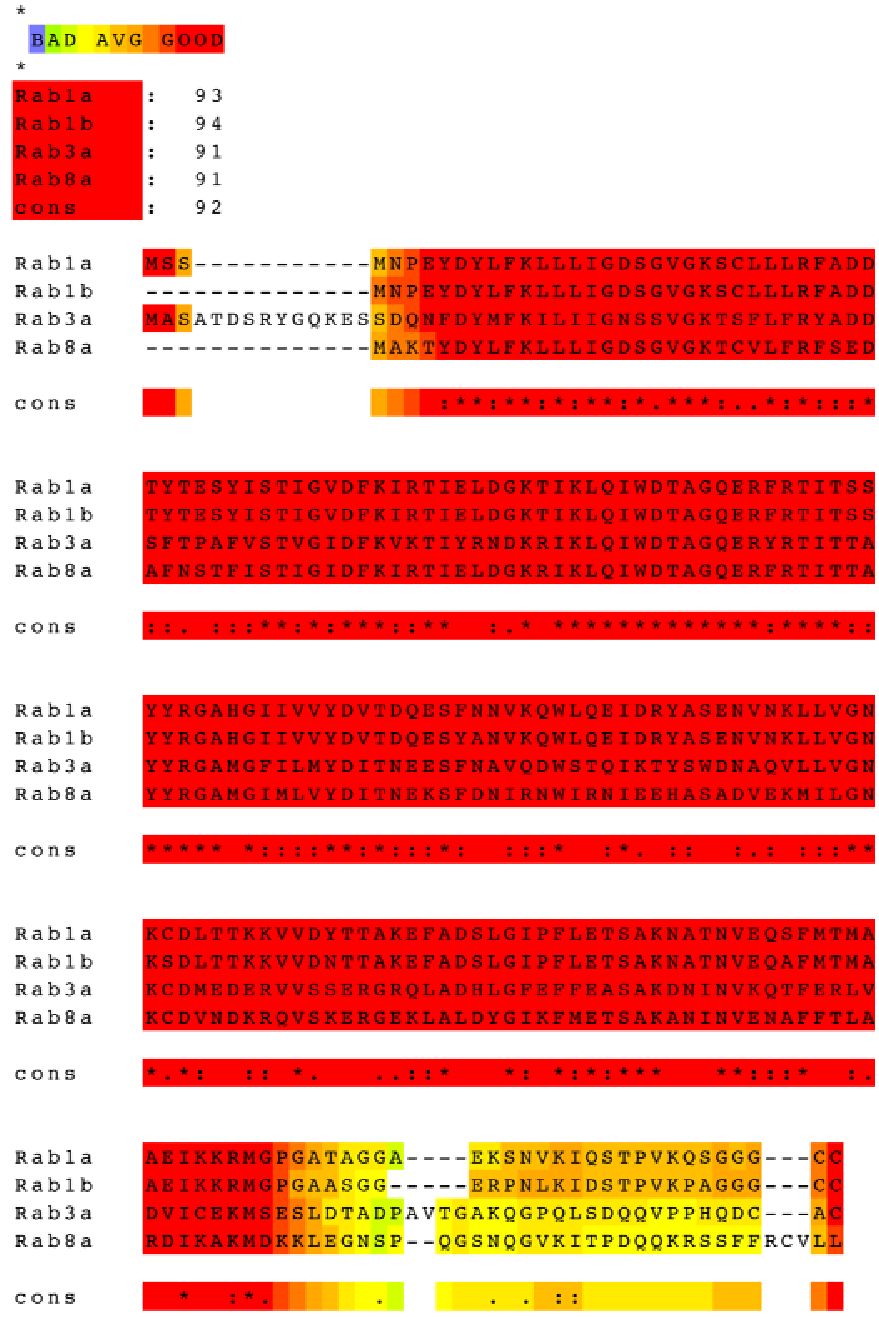

Figure 3.2.1 Sequence alignment for Rab1a, Rab1b Rab3a and Rab8a. Multiple sequence alignment has been implemented for the four protein sequences (homo sapiens) by T-coffee method on the server (Notredame et al, 2000; Poirot et al, 2003). “*”: Identical, “:”:conserved substitution, “.”: semi-conserved substitution. The color scale indicates the scoring of alignment from blue (score $=0)$ to red (score $=100)$, where the intermediate score is indicated by range of yellow. 
Sequence alignment provides us a preliminary insight into the difference between the primary structures of 3 Rab proteins. As shown in Figure 3.2.1, Rabla and Rablb share very high degrees of similarity and identity of sequence in which only 14 residues differ, most of them are restricted to the range 180-199 (Touchot et al, 1989). Rab8a as the closet paralog to Rab1, also displays a high degree of similarity to Rab1a (75\% similarity and 55\% identity) and it mainly differs from Rab1 at at its C-terminal tail regarding its distinct physiological function on post-Golgi vesicles. Of these four Rab proteins, Rab3a shows the least similarity to the other three, particularly that its Nterminal possesses additional 11 residues that are not present in the other three proteins, accounting for the neuron-specific expression of Rab3a (Hutagalung \& Novick, 2011). Apart from the amino and carbonyl termini of these four proteins, the majority of the sequences corresponding to the folded parts of Rab proteins are highly similar. This is a common feature conserved in members of the Ras-GTPases family as this part is responsible for GTP hydrolysis (Itzen \& Goody, 2011).

\subsubsection{Unique binding of Rab8a to $\alpha$-Synuclein}

NMR signals of backbone amides provide excellent information to probe proteinprotein/ligand interactions with description of the interaction interface and binding affinity according to perturbation of chemical shift and line-width analysis (Craik \& Wilce, 1997; Gao et al, 2004). Thus, based on NMR spectroscopy, we studied interactions of $\alpha \mathrm{S}$ with different Rabs and identified the corresponding binding sites at $\alpha \mathrm{S}$.

\subsubsection{Rab8a, but not Rab1b and Rab3a binds $\alpha$-Synuclein}

The ${ }^{1} \mathrm{H}-{ }^{15} \mathrm{~N}$ HSQC spectra of $\alpha \mathrm{S}$ in the presence of Rablb, Rab3a, and Rab8a respectively for molar ratios up to $1: 10$ of $\alpha \mathrm{S}$ to the $\mathrm{Rab}$ were recorded at $15^{\circ} \mathrm{C}$ and $\mathrm{pH}$ 7.4. The resonances of the spectra for $\alpha \mathrm{S}$ were well resolved and sharp. Both GDP- and GppNHp-(a hydrolysis-resistant structural analogue to GTP) bound Rabs were titrated into $\alpha \mathrm{S}$.

As reflected in Figure 3.2.2(A), titration of unlabeled Rab8a (GDP- and GppNHp-) into isotropic ${ }^{15} \mathrm{~N}$-labeling $\alpha \mathrm{S}$ resulted in the shifting of the C-terminal resonances of $\alpha \mathrm{S}$. However, there were no significant bindings found in the cases of 
Rab1b and Rab3a. In the presence of Rab1b and Rab3a, the C-terminal and rest parts of $\alpha \mathrm{S}$ did not show a clear trend of chemical shift perturbation. In the presence of Rab8a, the affected residues of $\alpha \mathrm{S}$ with chemical shift perturbation beyond $0.06 \mathrm{ppm}$ were mainly located at the acidic C-terminal region from G111 to A140. The rest parts of the $\mathrm{N}$-terminus and NAC region of $\alpha \mathrm{S}$ remain unperturbed by the presence of Rab8a. Displacement of $\alpha \mathrm{S}$ peak positions was demonstrated as the function of stepwise addition of Rab8a(GDP) and Rab8a(GppNHp) [Figure3.2.2 (B), (C)]. Neighboring regions of residue Y125 and D134 of $\alpha \mathrm{S}$ were mostly influenced by an increasing molar ratio of Rab8a to $\alpha$ S. Both GDP- and GppNHp-bound Rab8a displayed similar binding pattern to $\alpha \mathrm{S}$ but different affinities.

Based on the chemical shift perturbation of $\alpha \mathrm{S}$ at different amounts of Rab8a, the $\mathrm{K}_{\mathrm{d}}$ values for Rab8a(GDP) and Rab8a(GppNHp) binding to $\alpha \mathrm{S}$ were estimated as $0.186902 \pm 0.008463 \mathrm{mM}$ and $0.45089 \pm 0.045431 \mathrm{mM}$ respectively. Indeed, quantitative comparison represented by $\mathrm{K}_{\mathrm{d}}$ demonstrated that GDP-bound Rab8a possessed stronger binding affinity to $\alpha \mathrm{S}$ than the GppNHp-bound Rab8a, consistent with the observation of titration [Figure3.2.2 (B), (C)]. The $\mathrm{K}_{\mathrm{d}}$ difference in these two states would have importantly functional implication regarding to the interplay between $\alpha \mathrm{S}$ and Rab8a. Hence, in the following analysis, we mechanistically dissected this interaction to identify the binding sites at both proteins in detail. 

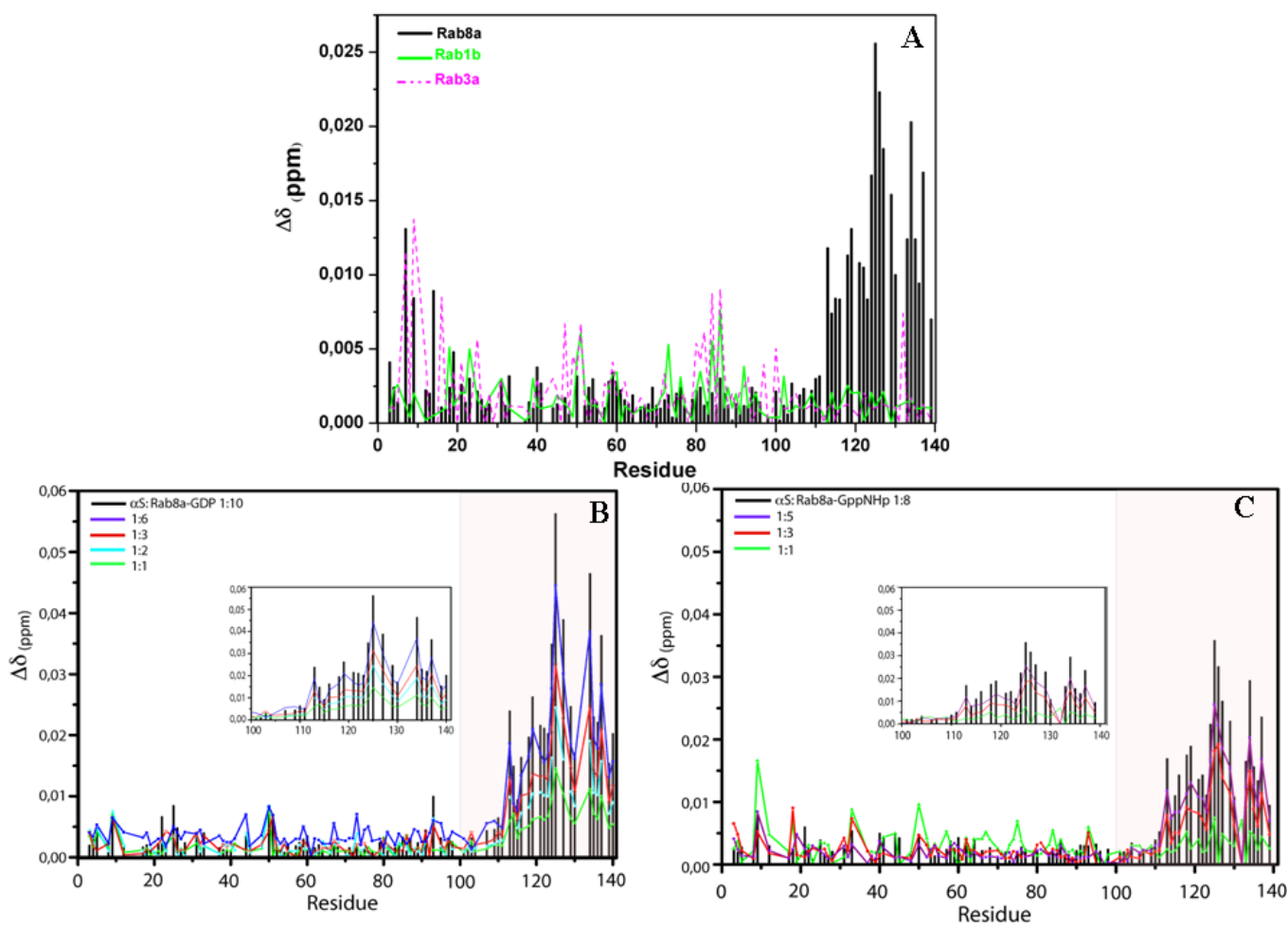

Figure 3.2.2 NMR titration of Rab1b, Rab3a and Rab8a into ${ }^{15} \mathrm{~N}-\alpha \mathrm{S}$. (A)Chemical shift perturbation for studying protein interaction was shown by defining resonance shifting in NMR HSQC spectra of $\alpha$, which were in the presence of Rab GTPases at the concentration ratio 1 to 5 (black bar: Rab8a, green line: Rab1b and purple dash line: Rab3a). Replacement of the affected peaks of $\alpha \mathrm{S}$ was increased as the function of added concentrations of Rab8a at GDP-bound state (B) and GppNHp-bound state (C). (The HSQC spectra were recorded by María L. Orcellet.)

\subsubsection{Anatomy of the interaction between $\alpha$-Synuclein and Rab8a}

$\alpha \mathrm{S}$ consists of three functional domains, namely the N-terminus, the NAC region and the C-terminus. Each of these three domains has been well-characterized with distinct features with respect to monomeric structure, fibril formation, molecular modification, membrane affinity, ligand binding etc (Bisaglia et al, 2009). It's therefore necessary to determine the structural reason why Rab8a specifically binds to C-terminal part of $\alpha \mathrm{S}$ (residue110-140).

Compared with other two domains, negatively charged residues (Glu and Asp) are enriched in the C-terminal part of $\alpha \mathrm{S}$. Such significant number of negative charges contributes to the long-range interactions and stabilizes the tertiary conformation of $\alpha \mathrm{S}$ (Bertoncini et al, 2005b; Dedmon et al, 2005c). Meanwhile, the abundant negative 
charges offer the opportunity for positively charged binding partners to interact with the C-terminal of $\alpha \mathrm{S}$, which was found in the case of polyamines binding (Fernandez et al, 2004). It's has also been noticed that the members of the Rab family differs mainly at their C-termini. Based on this fact, we firstly analyzed the sequence of $\alpha \mathrm{S}$ and the $3 \mathrm{Rab}$ proteins [Figure 3.2.3]. Unsurprisingly, a significant number of negative charges "-13" are distributed at C-terminal part (100-140) of $\alpha \mathrm{S}$ and Rab8a is the only one possessing positive net-charge “+3” at its C-terminus. However, Rab1b and Rab3a possess rather neutral or even negative charges at their C-termini respectively. Therefore, the observed binding of Rab8a to $\alpha \mathrm{S}$ by NMR titration occurs probably as a result of charge-driven electrostatic interaction between C-termini of the two proteins.

\section{Charge distribution in the C-terminals \\ Rab1b: --G ${ }^{176}$ AASGGERPNLKIDSTPVKPAGGGCC ${ }^{201}$ \\ Rab3a: --S ${ }^{188} E$ SLDTADPAVTGAKQGPQLSDQQVPPHQDCAC 220 \\ Rab8a: --D ${ }^{176}$ KKLEGNSPQGSNQGVKITPDQQKRSSFFR ${ }^{205}$ CVLL $^{209}$ \\ $\alpha S \quad:--{ }^{100}$ GKNEEGAPQEGILEDMPVDPDNEAYEMPSEEGYQDYEPEA ${ }^{140}$ \\ $\square$ Positive charge $\square$ Negative charge}

Figure3.2.3. Charge distribution of C-terminal residues of Rab1b, Rab3a, Rab8a and $\alpha$ S. The charge distribution has been demonstrated by labeling the charged residue by specified color (red: positive charge, cyan: negative charge). The significant positive net-charge of Rab8a and negative netcharge of $\alpha \mathrm{S}$ were unambiguously indicated.

According to the analysis of the charge distribution, we synthesized the Cterminal peptide of Rab8a comprising of 30 residues (Asp176-Arg205) and titrated it into $\alpha \mathrm{S}$. The six positively charged residues Lys177, Lys178, Lys192, Lys199, Arg200, Arg205 and three negatively charged residues Asp176, Glu180, Asp196 were included in this peptide and together were characterized as " +3 " net charges. The peptideinduced perturbations of chemical shifts to $\alpha \mathrm{S}$ resembled well the effect of full-length Rab8a on the C-terminal of $\alpha \mathrm{S}$ [Figure3.2.4]. The black bars in the figure represent the chemical perturbation at the molar ratio of Rab8a-GDP to $\alpha \mathrm{S}$ as $5: 1$, shown as a reference in this figure. The red line indicates the chemical perturbation of $\alpha \mathrm{S}$ at 10:1 ratio of peptide: $\alpha \mathrm{S}$. The pattern and the magnitude of perturbation of the peptide is similar to full-length Rab8a. But, the amount of peptide used is twice the amount of the 
full-length protein, suggesting that full-length protein maintain a stronger affinity to $\alpha \mathrm{S}$ than its C-terminal fraction.

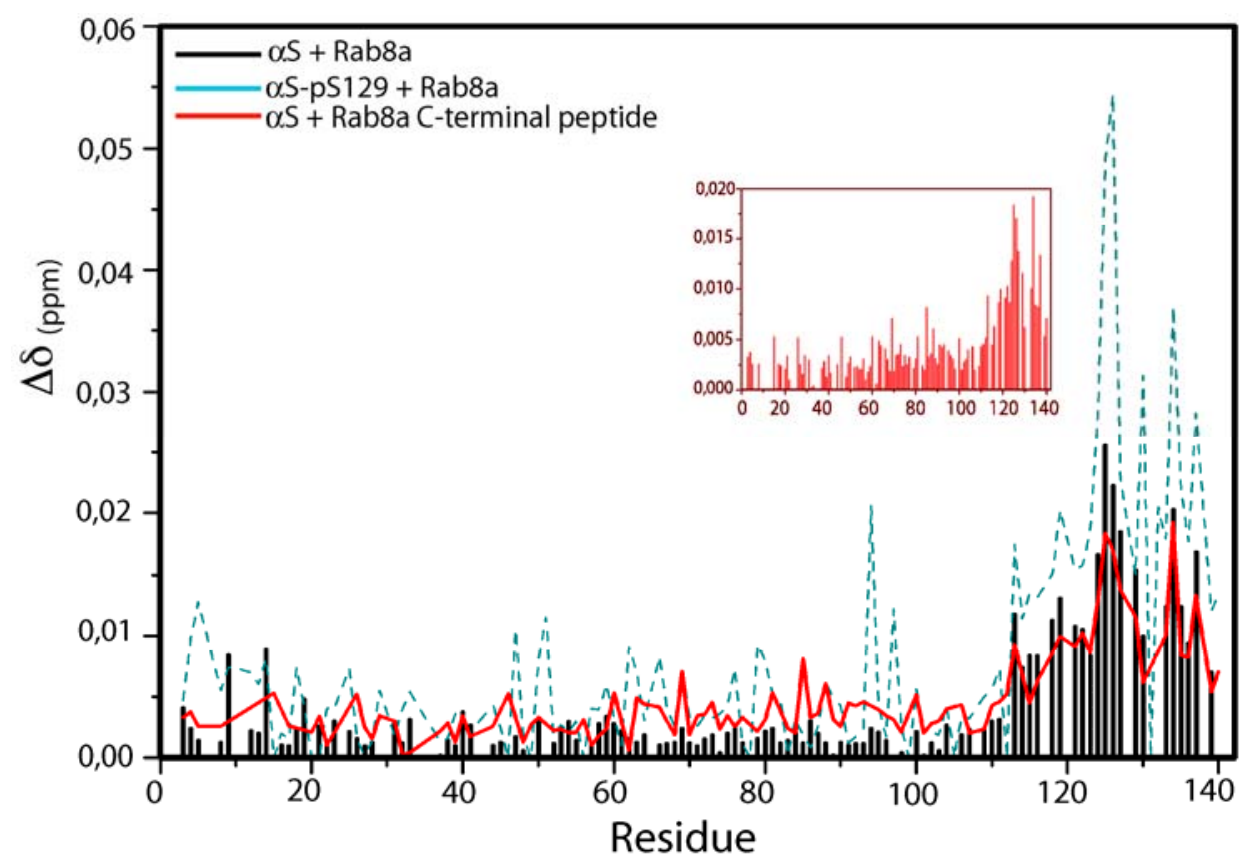

Figure 3.2.4. Interaction between C-termini of $\alpha \mathrm{S}$ and Rab8a. Chemical shift perturbations show the binding between the C-termini of Rab8a and $\alpha \mathrm{S}$. $\alpha \mathrm{S}$ (Black bar) and phosphorylated-Ser129 $\alpha \mathrm{S}$ (cyan dash line) were titrated by Rab8a at $\alpha \mathrm{S}$ :Rab8a ratio of 1:5. Meanwhile, $\alpha \mathrm{S}$ was titrated by Rab8a C-terminal peptide at $\alpha \mathrm{S}$ to peptide ratio of 1:10 (red line) and the inlet represents the corresponding chemical shift perturbation plot of the peptide titration.

Phosphorylated Ser129 $\alpha$ S was generated by using Polo-Like Kinase 3 (PLK3) and the interaction between phosphorylated $\alpha \mathrm{S}$ and Rab8a was then assessed. Phosphorylated-Ser129 $\alpha \mathrm{S}$ displayed a similar binding model to $w t-\alpha \mathrm{S}$ but the degree of perturbation was enhanced by approximately a factor of approximately 2 due to the phosphorylation [Figure 3.2.4]. The phosphorylation at Ser129 introduced one more negative charge to $\mathrm{C}$-terminus of $\alpha \mathrm{S}$, which can be reflected by the increased perturbation around pSer129. Therefore, the opposite charges existing at the carboxyl termini of $\alpha \mathrm{S}$ and Rab8a play an important role in adjusting their interaction. 
Table 3.1 Statistics of charged residues for C-terminally truncated Rab1b, Rab3a and Rab8a

Rab1b(1-175)

Rab3a(1-187)

$\operatorname{Rab} 8 \mathrm{a}(1-173)$
Positively charged residues $(\mathrm{K}+\mathrm{R})$

21

23

27
Negatively charged

residues $(\mathrm{D}+\mathrm{E})$

24

27

24
Sum of charges (Netcharge)

$-3$

$-4$

$+3$

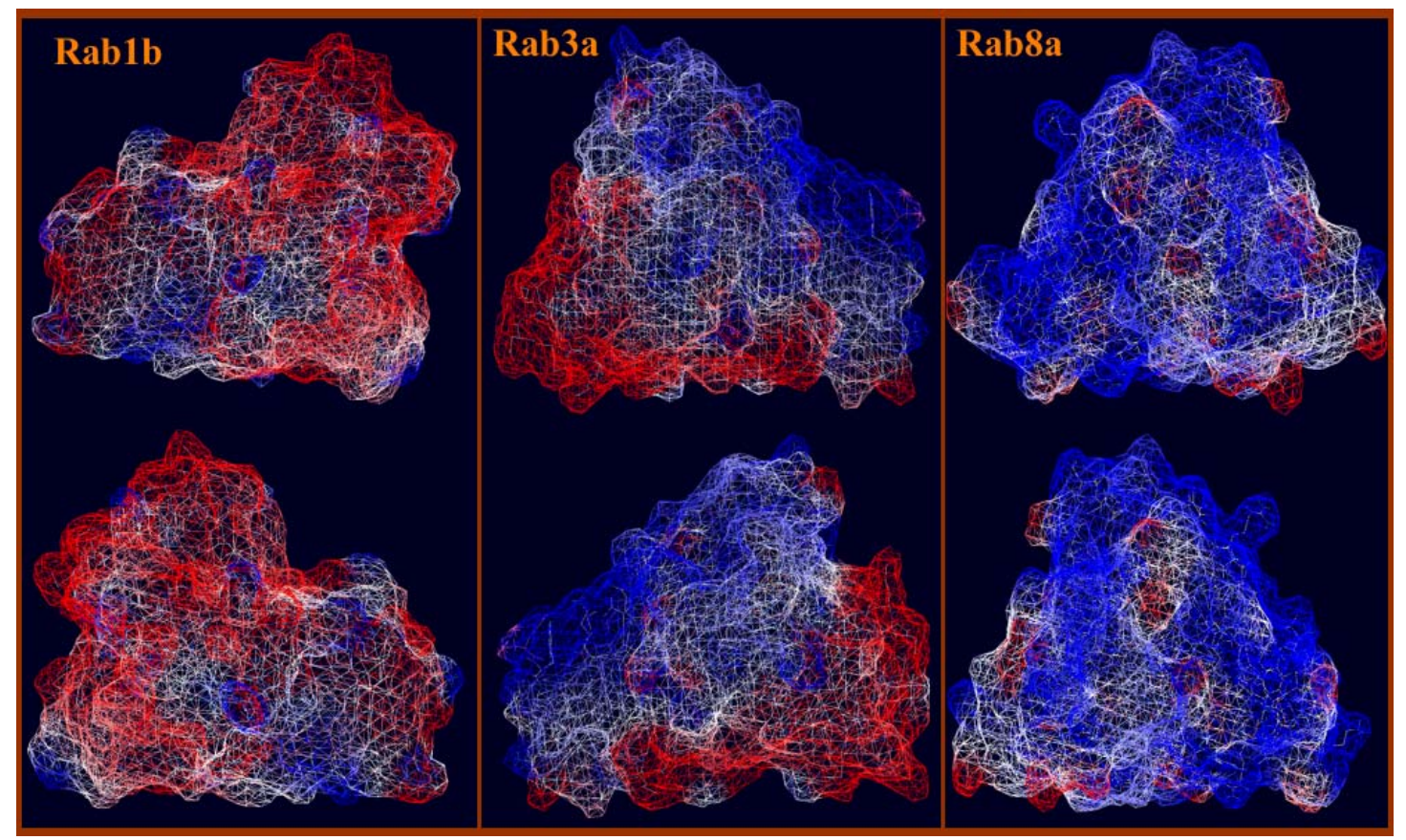

Figure 3.2.5. Surface mapping of electrostatic potential for C-terminally truncated Rab1b, Rab3a and Rab8a. Electrostatic potential based on the coulomb interactions was calculated by Swiss-PdbViewer (Guex \& Peitsch, 1997). The structures of Rab1b (PDB code: 3NKV), Rab3a (PDB code:3RAB) and Rab8a (PDB code: 3QBT) were chosen for the surface mapping (Dumas et al, 1999; Hou et al, 2011; Muller et al, 2010). Each structure with the surface mapping was displayed with the view of both facing and back. The blue color stands for positive potential, whereas red color and white color stand for negative potential and neutral potential.

The positively charged C-terminal peptide can resemble the effect of full-length Rab8a binding to $\alpha \mathrm{S}$, but has a relatively weaker affinity of this peptide to $\alpha \mathrm{S}$ than the full-length protein has been recognized. This suggests that except for the C-terminal tail, other parts of Rab8a are capable of binding to C-terminus of $\alpha \mathrm{S}$ as well. Therefore, we investigated the charge states of the folded parts of these $3 \mathrm{Rab}$ proteins. The statistic of charge states for the C-terminally truncated parts of 3 Rabs has been performed by the ExPASy Protparam Tool (Artimo et al, 2012) [Table 3.1]. By counting the number of 
positively and negatively charged residues in the $\mathrm{N}$-terminal folded part, the total charge of Rab8a is positive 3, contrary to the -3 for Rablb and the -4 for Rab3a. This suggests that the folded part of Rab8a also has potential to bind to the C-terminal of $\alpha \mathrm{S}$. Furthermore, surface mapping by electrostatic potential was performed for the published crystal structures of these three Rab proteins [Figure 3.2.5]. In line with the charge statistics described in Table3.1, the surface of Rab8a largely presents positively electrostatic potential which indicates that the outer surface of Rab8a is dominantly occupied by positively charged residues. Whereas, Rab3a shows relatively neutral surface and Rab1b presents more negative potential on its surface. In conclusions, charge statistics and surface potential mapping suggests that $\mathrm{N}$-terminally folded part of Rab8a possesses a strong propensity to bind to the highly negative C-terminus of $\alpha \mathrm{S}$.

\subsubsection{C-terminally truncated Rab8a binds to $\alpha$-Synuclein}

Rab8a(6-176) with the truncation of the C-terminal tail (Rab8a- $\delta C)$ has been used to study its potential to interacting with $\alpha$ S. Both Rab8a- $\delta$ C(GDP) and Rab8a$\delta \mathrm{C}(\mathrm{GppNHp})$ were titrated into ${ }^{15} \mathrm{~N}-\alpha \mathrm{S}$ and changes of $\alpha \mathrm{S}$ were reflected by crosspeaks of backbone ${ }^{1} \mathrm{H}-{ }^{15} \mathrm{~N}$ resonance of $\alpha \mathrm{S}$ in two dimensional HSQC spectra [Figure3.2.6]. Both Rab8a- $\delta \mathrm{C}(\mathrm{GDP})$ and $\mathrm{Rab} 8 \mathrm{a}-\delta \mathrm{C}(\mathrm{GppNHp})$ reproduced the binding effects to the acidic C-terminus(G111-A140) of $\alpha \mathrm{S}$ similarly to the full-length protein and the C-terminal peptide described before. Interestingly, the change of resonance positions illustrates that the $\mathrm{Rab} 8 \mathrm{a}-\delta \mathrm{C}(\mathrm{GDP})$ binds to $\alpha \mathrm{S}$ with a stronger affinity than $\mathrm{Rab} 8 \mathrm{a}-\delta \mathrm{C}(\mathrm{GppNHp})$ at the same molar ratio to $\alpha \mathrm{S}$. 

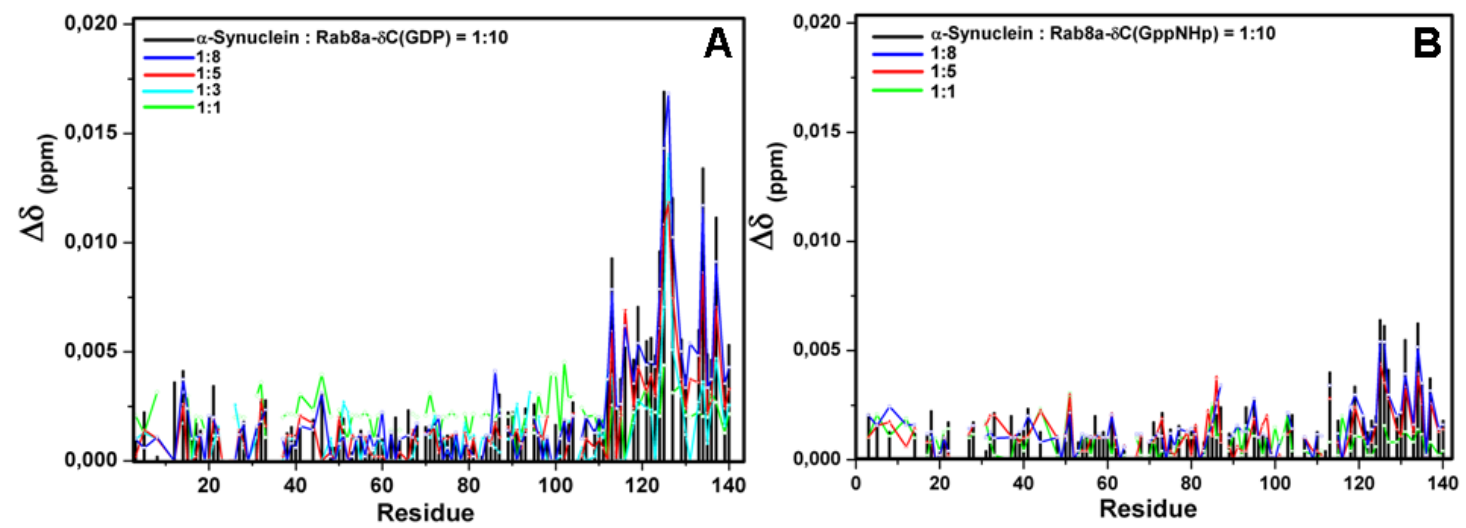

Figure 3.2.6. Analysis of Rab8a(6-176) binding to $\alpha \mathrm{S}$ by NMR spectroscopy. Changes in individual cross-peak positions of backbone ${ }^{1} \mathrm{H}^{15} \mathrm{~N}$ resonance of $\alpha \mathrm{S}$ in two dimensional HSQC spectra in the presence of Rab8a(6-176)-GDP(A) and Rab8a(6-176)-GppNHp(B) for the molar ratios up to 1:10 of $\alpha \mathrm{S} / \mathrm{Rab}$.

To clearly represent the different affinities, the changes of the peak positions in HSQC spectra of $\alpha \mathrm{S}$ in presence of molar ratio 1:1, 1:5, 1:10 $\alpha \mathrm{S} / \mathrm{Rab} 8 \mathrm{a}-\delta \mathrm{C}(\mathrm{GDP})$ and Rab8a- $\delta \mathrm{C}(\mathrm{GppNHp})$ have been selectively displayed and compared in Figure3.2.7. At molar ratio 1:1, the presence of both Rab8a-8C(GDP) [Figure3.2.7(A)] and the Rab8a$\delta \mathrm{C}(\mathrm{GppNHp})$ [Figure3.2.7(B)] did not induce obvious changes in $\alpha \mathrm{S}$. At the ratio of 1:5, both samples shows the binding to the C-terminal of $\alpha \mathrm{S}$. But, the GDP-bound form [Figure3.2.7(C)] already shows $\sim 3$-fold larger magnitidue of resonance shifts as the GppNHp-bound form [Figure3.2.7(D)] and this trend remains until the molar ratio of Rab8a- $\delta \mathrm{C}$ to $\alpha \mathrm{S}$ reached 10:1 for the GDP-bound form [Figure3.2.7(E)] and GppNHpbound form [Figure3.2.7(F)]. The higher affinity of Rab8a- $\delta \mathrm{C}(\mathrm{GDP})$ than Rab8a$\delta \mathrm{C}(\mathrm{GppNHp})$ for binding to $\alpha \mathrm{S}$ was also demonstrated by the intensity plot at the Rab8a- $\delta \mathrm{C}$ to $\alpha \mathrm{S}$ molar ration of 10:1 [Figure3.2.7 (G) and $(\mathrm{H})]$. 

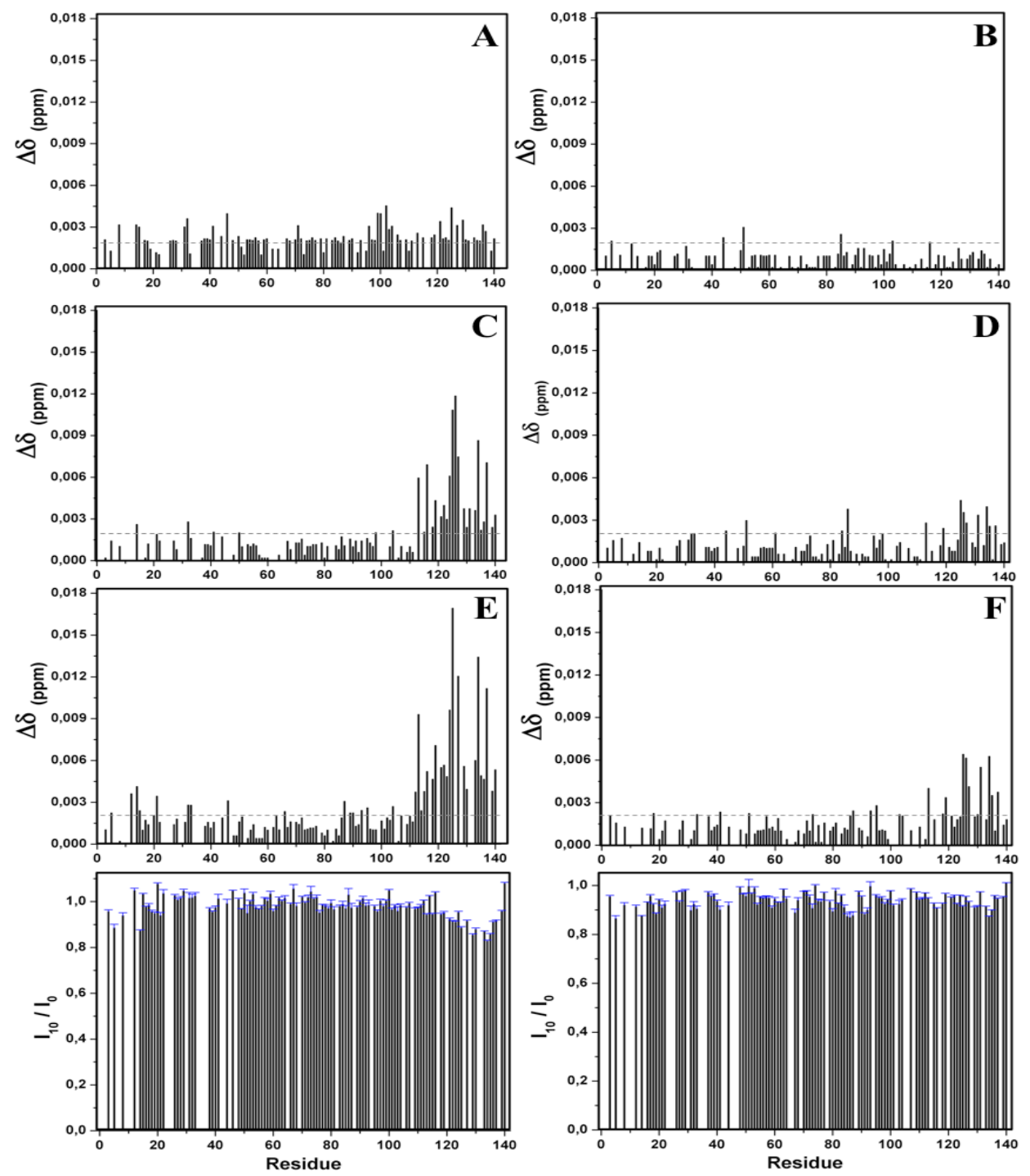

Figure3.2.7 Analytic comparison for Rab8a(6-176)-GDP and Rab8a(6-176)-GppNHp binding to $\alpha$ S.

Change in chemical shifts of individual HSQC peaks of $\alpha \mathrm{S}$ in the presence of Rab8a(6-176)-GDP (A, C, E) and $\operatorname{Rab8a}(6-176)-\operatorname{GppNHp}(\mathrm{B}, \mathrm{D}, \mathrm{F})$ at molar ratio of 1:1, 1:5, 1:10 of $\alpha \mathrm{S}$ to Rab8a(6-176). The dashline in chemical shift perturbation plots indicates average noise level as estimated by comparing repeating experiments. The intensities of cross peaks of $\alpha \mathrm{S}$ at 1:10 molar ratio to Rab8a(6-176) have been shown in $(\mathrm{G})$ and $(\mathrm{H})$ for GDP and GppNHp bound Rab8a(6-176). Error bars were calculated based on signal to noise.

In summary, we found that the electrostatic interactions existing between $\alpha \mathrm{S}$ and Rab8a were important for the binding of Rab8a to $\alpha \mathrm{S}$. This was further confirmed by the fact that Ser129 phosphorylation of $\alpha \mathrm{S}$ enhances the binding remarkably and that the C-terminus peptide of Rab8a can reproduce the binding to $\alpha \mathrm{S}$. Furthermore, not only the C-terminus but also the N-terminally folded part of Rab8a possesses capacity 
to bind to $\alpha \mathrm{S}$, indicated by surface mapping of its crystal structure. NMR titration of Rab8a- $\delta \mathrm{C}$ into $\alpha \mathrm{S}$ proved that this truncated Rab8a could bind to $\alpha \mathrm{S}$. We also observed a different affinity of GDP- and GppNHp- bound Rab8a- $\delta \mathrm{C}$ to $\alpha \mathrm{S}$, consistent with the results of full-length Rab8a.

\subsubsection{Aggregation assays of $\alpha-S y n u c l e i n$ with Rab8a and it's C-terminus}

Following the identification of Rab8a/ $\alpha \mathrm{S}$ interaction by NMR, we investigated the role of Rab8a in mediating $\alpha \mathrm{S}$ aggregation by ThT fluorescence. Linking with the NMR interaction assays, $\alpha \mathrm{S}$ was incubated with full-length Rab8a(GDP) and the C-terminus peptide of Rab8a respectively under the aggregation conditions. Firstly, Rab8a displayed a distinctive efficiency in modulating $\alpha \mathrm{S}$ aggregation [Figure 3.2.9. (A)]. For the applied molar ratio of Rab8a to $\alpha \mathrm{S}$, all 1:0.3, 1:1, 1:3 ratios revealed that substantial enhancement of fibril formation was achieved by shortening the lag phase time and reaching the saturated stage more rapidly than in the absence of Rab8a. Interestingly, however, when the molar ratio was raised up to $5: 1$ of Rab8a to $\alpha \mathrm{S}$, the profile of aggregation kinetics was different to the cases with lower amount of Rab8a. At this ratio, no kinetic improvement was observed and the fibril growth displayed similar trend to the $\alpha \mathrm{S}$ control without the addition of Rab8a.

Following the aggregation, EM was used to distinguish between the different fibrillar species generated under the different molar ratios of Rab8a to $\alpha \mathrm{S}$ [Figure 3.2.9. (B)-(D)]. Referring to the characteristic morphology displayed by the fibrils of $\alpha \mathrm{S}$ control, EM images addressed the increased protein backgrounds accumulated on the surface of fibrils as a consequence of increased amounts of Rab8a. With the increased amount of Rab8a, morphological diversity and the content of fibril association were also enlarged, particularly for the circumstances where the amount of Rab8a exceeded $\alpha \mathrm{S}$. This protein background is likely as a result of the Rab8a attaching to the fibril surface by binding to the $\alpha \mathrm{S}$ C-terminus. The C-terminus of $\alpha \mathrm{S}$ retains its disordered conformation outside the fibril core and thus is readily recognized by binding partners, i.e. Rab8a. We also observed the precipitates in all trials in the presence of Rab8a. The amount of precipitates was well correlated with the amount of Rab8a added at the starting of aggregation as depicted in Figure 3.2.9 (B) - (D). This observation indicates 
that the observed precipitates were from the amorphous aggregates formed by Rab8a during the time course of $\alpha \mathrm{S}$ aggregation. Rab8a was reported to possess low stability and solubility under some expression conditions (Bleimling et al, 2009; Lim et al, 2011). Additionally, recent study shows that $\alpha \mathrm{S}$ can cause the aggregation of many different Rab GTPases in yeast and additionally the aggregation of $\alpha \mathrm{S}$ can also be enhanced (Lim et al, 2011).
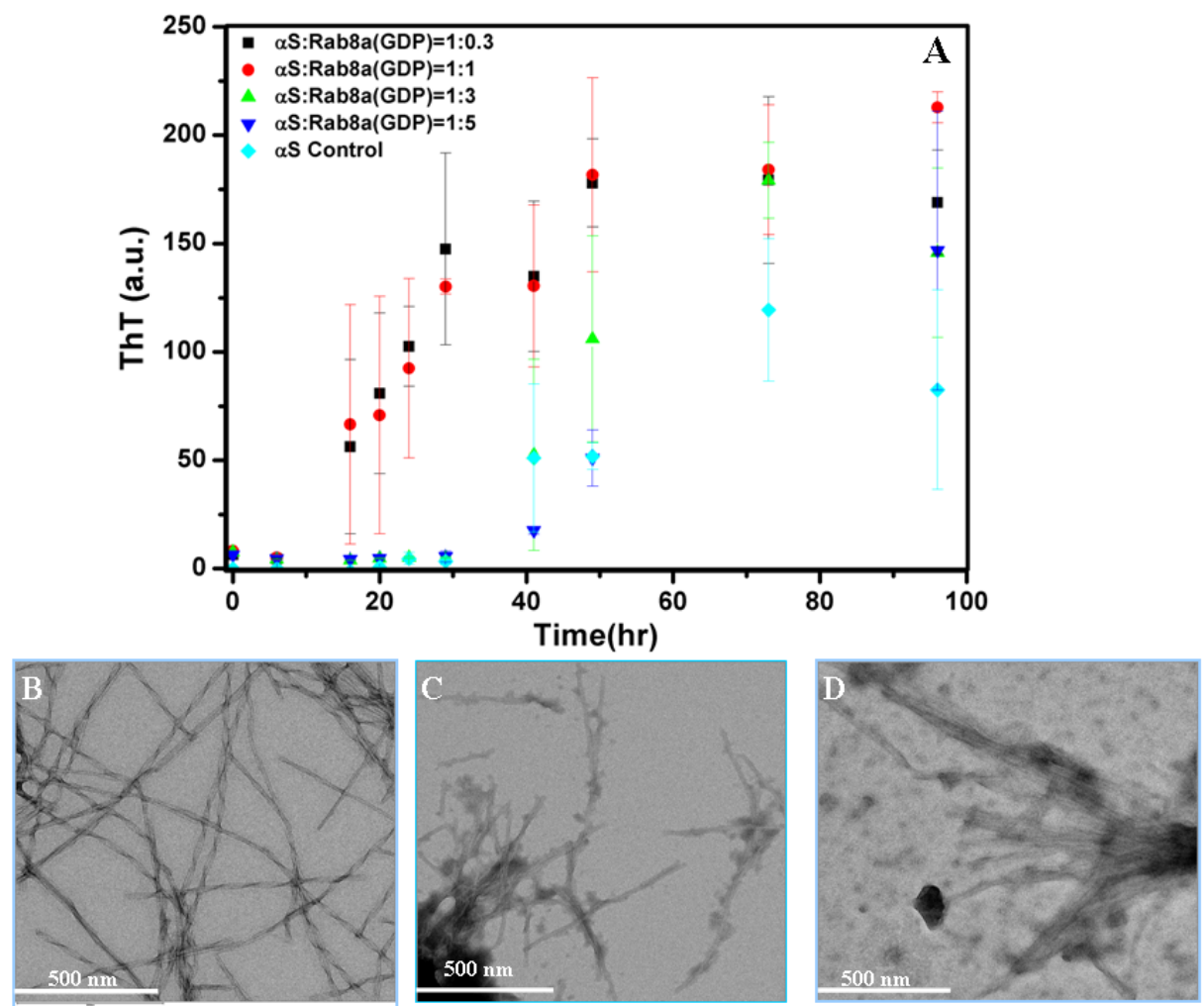

Figure 3.2.9. Aggregation assays of $\alpha \mathrm{S}$ in the presence of Rab8a. (A) $\alpha \mathrm{S}$ aggregations were influenced by Rab8a reflected by ThT fluorescence at $\alpha \mathrm{S}:$ Rab8a ratio of 1:0.3, 1:1, 1:3, 1:5. The corresponding EM images for the $\alpha \mathrm{S}$ control, 1:1 and 1:3 molar ratios are shown in figure (B) - (D), which illustrate both the amorphous background associated with Rab8a aggregates increased and the $\alpha \mathrm{S}$ fibrils with distinct morphology decreased as the function of present amounts of Rab8a.

In line with the observation that the C-terminus of Rab8a simulated the binding of full-length Rab8a to $\alpha \mathrm{S}$, this segment can also contribute to the mediating effects of Rab8a on $\alpha \mathrm{S}$ aggregation. The synthesized peptide containing 30 residues (174-205) of Rab8a C-terminal used in binding study was adapted in the aggregation assay by using 3 different molar ratios to $\alpha \mathrm{S}(1: 1,3: 1,6: 1)$. This peptide showed a does-independent 
manner of enhancement to $\alpha \mathrm{S}$ aggregation [Figure 3.2.10]. The comparison of kinetics of $\alpha \mathrm{S}$ aggregations in the presence of Rab8a and its C-terminal fraction reflects that the peptide shortened lag phase to a similar extent to the whole protein, but prompted the fibril elongation by a relatively weaker efficiency than the full-length protein. Threfore, we found that the carboxyl terminus participates in the functions played by intact Rab8a on binding to $\alpha \mathrm{S}$ and improving $\alpha \mathrm{S}$ aggregation.

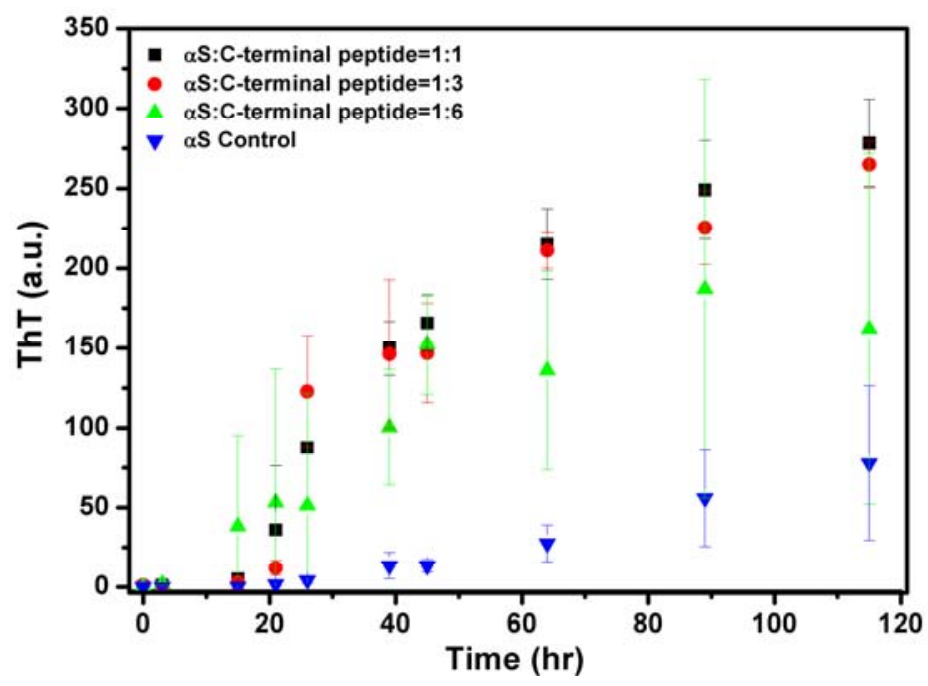

Figure 3.2.10. The C-terminal peptide of Rab8a simulated the effects of full-length protein on $\alpha \mathrm{S}$ aggregation by enhancing the kinetics. The molar ratios of $\alpha$ S:peptide were set as 1:1, 1:3 and 1:6.

In conclusions, both Rab8a and its C-terminal peptide improve the aggregation of $\alpha \mathrm{S}$ by significantly shortening the lag phase and enhancing the elongation rate. In concert with the interaction studies by NMR, Rab8a adjusts $\alpha \mathrm{S}$ aggregation by binding to its C-terminus.

\subsubsection{Aggregation assays of $\alpha$-Synuclein with Rab1b and Rab3a}

Rab1 and Rab3a were suggested to suppress the neuronal toxicity induced by $\alpha \mathrm{S}$ in animal models (Gitler et al, 2008). However, we did not observe the interactions of Rab1b or Rab3a with monomeric $\alpha \mathrm{S}$ in our NMR titrations. It's likely that these two Rab proteins selectively interact with $\alpha \mathrm{S}$ aggregates, i.e. oligomers and fibrils, rather than monomers. To address this question, we implemented aggregation assays for $\alpha \mathrm{S}$ in 
the presence of Rab1b and Rab3a to identify effects of these two Rabs on $\alpha \mathrm{S}$ aggregation.

\subsubsection{Aggregation with Rablb}
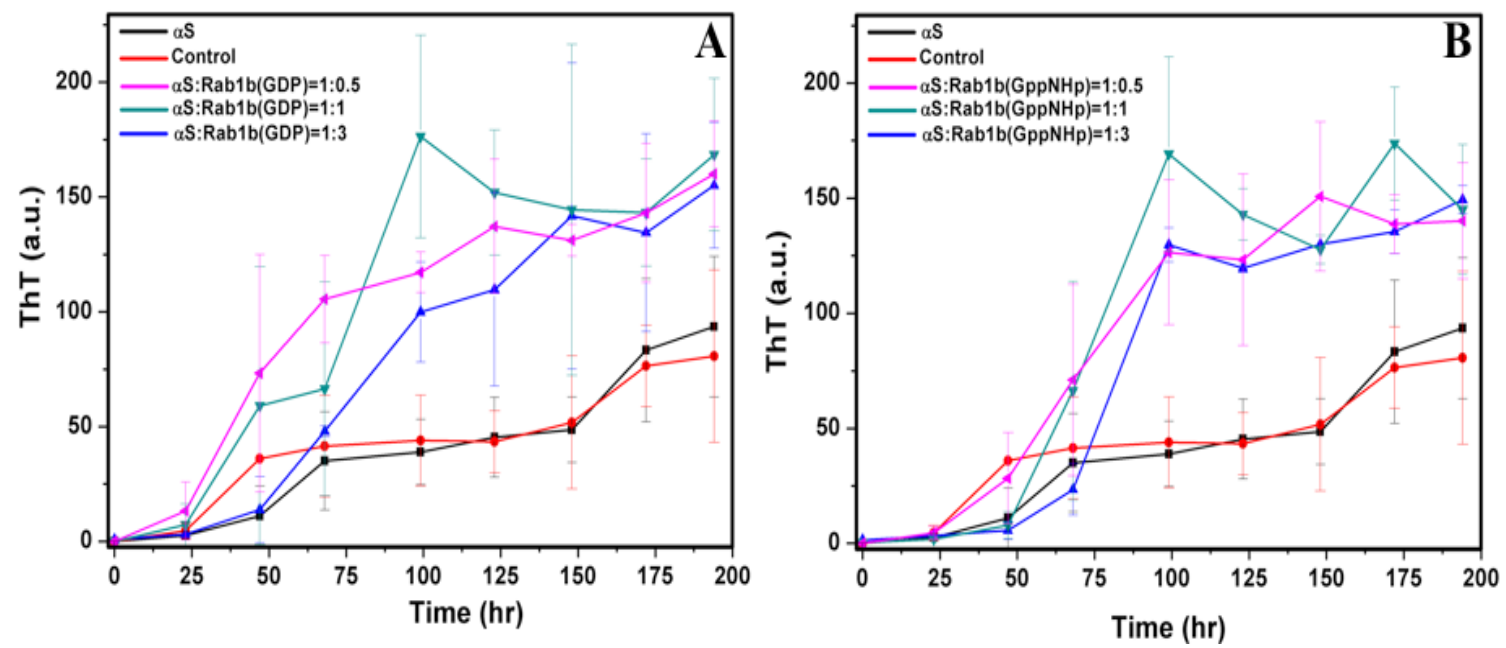

Figure 3.2.11. Aggregation assay of $\alpha \mathrm{S}$ in the presence of Rab 1b. Aggregation kinetics of $\alpha \mathrm{S}$ was catalyzed by the presence of Rab1b. $\alpha$ S was incubated with Rab1b(GDP) and Rab1b(GppNHp) at molar ratio of $0.5: 1,1: 1,3: 1$ to $\alpha \mathrm{S}$ under aggregation conditions. The control here is including Rab buffer to examine its effects on $\alpha \mathrm{S}$ aggregation. Fibril formation of $\alpha \mathrm{S}$ followed by Thioflavin $\mathrm{T}$ fluorescence. The starting concentration of $\alpha \mathrm{S}$ is $50 \mu \mathrm{M}$, and the temperature for aggregation was $21^{\circ} \mathrm{C}$.

As shown in Figure 3.2.11, the characteristic lag time of $\alpha \mathrm{S}$ aggregation was slightly reduced due to the addition of Rablb. Moreover, the elongation rate as an index highly correlated with the fibril growth rate was increased remarkably and thereby the time spanning for reaching saturation of fibril assembly was reduced by one half compared with $\alpha \mathrm{S}$ alone. Interestingly, not only the modification of kinetics was observed, but the yields of fibril product mirrored by ThT intensity during the saturation stage were also amplified by a factor of 2 in the presence of Rab1b, which illustrated that the fibrillization was substantially improved by Rablb. Additionally, EM images showed the fibrils generated with additions of Rab1b(GDP) and Rablb(GppNHp) represent more twisted or bundled assemblies of protofilaments, which is different from the canonical morphology of $\alpha \mathrm{S}$ fibrils as more single and extended forms.

Furthermore, the kinetic data highlighted that the fact that the presence of varied molar ratios of Rablb to $\alpha \mathrm{S}$ however did not lead to pronounced kinetic variance, 
indicating that Rablb mediated the aggregation by a dose-independent manner. The potential physiologically relevant differences between the active and inactive state of $\mathrm{Rab}$ is of major interests in our research. To this end, both Rab1b(GDP) and Rab1b(GppNHp) were adapted in the aggregation assays. However, there was no discrepancy in aggregation behaviors between the samples that modulated by the two forms of Rablb. For Rab proteins, as members of GTPase family, the buffer used to dissolve Rabs contains nucleotide (GDP, GTP or its analogue) and ion $\mathrm{Mg}^{2+}$. The aggregation of $\alpha \mathrm{S}$ with only Rablb buffer but without the protein was exerted as a control to examine the effects of these co-existing small molecules on aggregation. The kinetic of the Rab-buffer control kept same pattern as the $\alpha \mathrm{S}$ alone, suggesting that the nucleotides and $\mathrm{Mg}^{2+}$ at the concentration used this assay did not interfere with $\alpha \mathrm{S}$ aggregation.

\subsubsection{Co-aggregation control with BSA}

Besides Rab1b, globular proteins, such as protein chaperon HSP70 and HSP90, have been reported to effect on $\alpha \mathrm{S}$ aggregation (Dedmon et al, 2005b; Falsone et al, 2009; Roodveldt et al, 2009). For the case of HSP70 in particular there were no direct interactions observed between this chaperon and monomeric $\alpha \mathrm{S}$ either, but it did modify $\alpha \mathrm{S}$ aggregation in an ATP-dependent manner. Therefore, does coexistence of globular proteins have generic impact on $\alpha \mathrm{S}$ aggregation? To clarify this point, we placed protein BSA (Bovine Serum Albumin) as a globular protein control into $\alpha \mathrm{S}$ aggregation.

Under the aggregation conditions, only the presence of Rab1b-GDP distinctively improves aggregation of $\alpha \mathrm{S}$ [Figure3.2.12]. The presence of BSA slightly shortened the lag phase but it did not simulate the function of Rab1b in promoting fibril growth. Pure BSA and Rab1b did not generate the ThT-active fluorescence throughout 90 hours of aggregation time. Thus, in comparing with BSA, Rab1b significantly enhances $\alpha \mathrm{S}$ aggregation with respect to fibril growth. We can thereby conclude that the aggregation improvement we observed in the previous assays was specifically as the result of Rablb. 


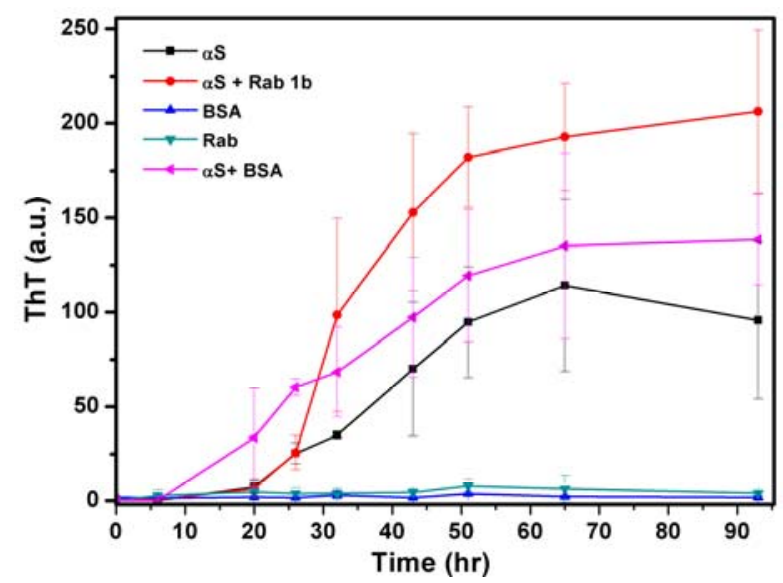

Figure 3.2.12. Co-aggregation control of $\alpha \mathrm{S}$ with Bovine serum albumin (BSA). $\alpha \mathrm{S}$ was aggregated with BSA and Rab1b(GDP) respectively at equal molar ratio. BSA and Rab1b-GDP were also incubated alone under the aggregation condition. The starting concentration of $\alpha \mathrm{S}$ was $40 \mu \mathrm{M}$, the concentrations of BSA and Rablb(GDP) were $4 \mu \mathrm{M}$. The temperature for aggregation was $21^{\circ} \mathrm{C}$.

\subsubsection{Aggregation with Rab3a}

Rab3a has highly neuron-specific expression and it can suppress the neuronal toxicity of $\alpha \mathrm{S}$ in the animal models (Gitler et al, 2008). At the same time, similar to Rab3a, Rab1b doesn't bind to monomeric $\alpha \mathrm{S}$ but it does significantly enhance $\alpha \mathrm{S}$ aggregation. Therefore, we investigated whether Rab3a has a similar effects on $\alpha \mathrm{S}$ aggregation.

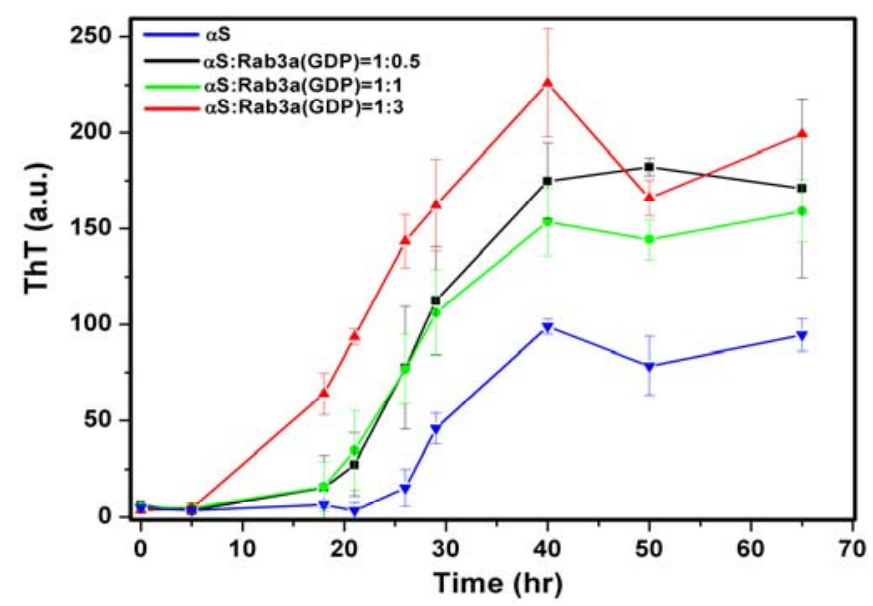

Figure 3.2.13. $\alpha \mathrm{S}$ aggregation in the presence of Rab3a. $\alpha \mathrm{S}$ : Rab3a ratios of 1:0.5, 1:1, 1:3 were adopted and compared to the control of $\alpha \mathrm{S}$ alone. As Rab1b and Rab8a, Rab3a could effectively enhance $\alpha \mathrm{S}$ aggregation at all 3 ratios and when it reached the ratio of 3:1 of Rab8a to $\alpha \mathrm{S}$, the aggregation kinetics was significantly modified. 
Under the aggregation condition, the $\alpha \mathrm{S}$ control displayed a typical lag phase more than 20 hours and reached the saturation phase at 40 hours of experimental time. In contrast, the aggregation kinetics was significantly accelerated by the presence of Rab3a-GDP at all 3 molar ratios. At the time point of 18 hours, all the trials with Rab3a already ended the lag phase and started the elongation. Similar to the control, the kinetics of $\alpha \mathrm{S}$ aggregation with Rab3a achieved saturation around 40 hours, but the fibril yields of the all 3 ratios characterized by ThT fluorescence were shown as twice as great as the control. Moreover, there is no dose-dependence observed between the molar ratio $\alpha \mathrm{S}$ to $\mathrm{Rab}$ of $1: 0.5$ and $1: 1$. For the molar ratio of $1: 3$, the lage phase was significantly shortened. And the elongation rate of $\alpha \mathrm{S}$ showed an even faster rate than the other ratios, but finally remains at a similar level of fibril yield as the other two molar ratios. Therefore, Rab3a enhances $\alpha \mathrm{S}$ aggregation in a manner similarly to Rab1b. Furthermore, unlike Rab8a, both Rab1b and Rab3a don't bind to monomeric $\alpha \mathrm{S}$ but they rather modulate aggregation of $\alpha \mathrm{S}$, suggesting that these two Rabs interact with $\alpha \mathrm{S}$ aggregates.

\subsubsection{NMR assignment of Rab1b and Rab8a}

In our studies described above, Rab1b, Rab3a and Rab8a have been shown to modulate $\alpha \mathrm{S}$ aggregation in a similar manner. However, according to the NMR titrations, only Rab8a binds to monomeric $\alpha \mathrm{S}$ whilst Rab1b and Rab3a do not. Hence, there two questions that arise following these observations: 1) How dose $\alpha \mathrm{S}$ bind to Rab8a and are there additional binding sites on Rab8a other than its C-terminal tail?; 2) Even if no bindings are observed, Rab1b and Rab3a still impacts on $\alpha \mathrm{S}$ aggregation. Does this implicate that they selectively interact with $\alpha \mathrm{S}$ aggregates.

Bearing these two questions, we performed NMR assignment for Rab1b and Rab8a. Rab1b(1-175)-GDP (Rab1b- $\delta$ C) and full-length Rab8a-GDP with uniformly ${ }^{13} \mathrm{C}$ and ${ }^{15} \mathrm{~N}$ labeling were prepared based on the optimized conditions achieved by Dr. Amyelt Itzen's group. Based on the assignment we obtained, the solution structures of these two proteins have been analyzed and the interaction studies related to $\alpha \mathrm{S}$ have been further implemented for these two Rab proteins. 


\subsubsection{Assignment and structural analysis of Rablb}

Uniformly ${ }^{13} \mathrm{C}$, ${ }^{15} \mathrm{~N}$-enriched Rab1b-GDP was prepared with a concentration of $1.5 \mathrm{mM}$ and used in the backbone NMR assignment of Rablb. The assignment was achieved by defining protein backbone sequential connectivity through the $\mathrm{C} \alpha / \mathrm{C} \beta$ moiety and $\mathrm{CO}$ moiety based on corresponding NMR spectra (Sattler et al, 1999). For assignment, triple resonance NMR experiments were applied based on transferring of coherence along the network of scalar coupled spins ${ }^{1} \mathrm{H},{ }^{13} \mathrm{C}$ and ${ }^{15} \mathrm{~N}$. 3D HNCACB and HNCA spectrums generated the resonances of both intra-residue and inter-residue $\mathrm{C} \alpha / \mathrm{C} \beta$. With aid of $3 \mathrm{D}$ $\mathrm{CBCAcoNH}$ that selectively presents the inter-residue resonance, the intra- and interpeaks were distinguished in the spectra and their chemical shifts were labeled for construction of sequential connectivity through $\mathrm{C} \alpha / \mathrm{C} \beta$. Similarly, NHCO and HNcaCO together provided the distinguished peaks of both intra- and inter- CO. Thereby, the sequential connectivity through $\mathrm{CO}$ was further established. For better resolution, all 3D spectra were recorded with TROSY-based fashions (Salzmann et al, 1998). The chemical shifts of distinguished $\mathrm{C} \alpha / \mathrm{C} \beta$ and $\mathrm{CO}$ were input into the automatic assignment program MARS (Jung \& Zweckstetter, 2004a; Jung \& Zweckstetter, 2004b). MARS achieved assignment by establishing sequential connectivity and propensity of secondary structure which was reflected by carbon chemical shifts. Finally, $80 \%$ backbone assignment for Rablb- $\delta \mathrm{C}(\mathrm{GDP})$ was reliably obtained.

As shown in Figure 3.2.14(A), the resonances are well dispersed and characteristic of folded proteins in ${ }^{1} \mathrm{H}-{ }^{15} \mathrm{~N}$ TROSY. Most of the resonances that appeared in the spectrum have been unambiguously assigned apart from the signals introduced by side chains. Meanwhile, the selected strips representing the resonances in the HNCACB spectrum are shown in Figure 3.2.14(B), where the sequential connectivity has been demonstrated by linking the intra and inter $\mathrm{C} \alpha$ and $\mathrm{C} \beta$ resonances of the fraction R49 to K56 of Rab1b- $\delta$ C. All the chemical shifts of assigned residues were shown in the appendix I.

Interestingly, one part of protein consisting of residue Thr65-Thr75 could not be assigned. Inspecting into the spectra, these residues were likely disappearing. Mapping this sequence onto published crystal structure, we found this part corresponding to the Switch II region. This region is highly dynamical and strongly affected by the 
nucleotides binding as a common feature of Ras-GTPases family (Lee et al, 2009). The Switch II region consists of one short $\alpha$-helix ( $\alpha 2)$ and one long disordered loop (G3). The crystal structure with B-factor mapping [Figure 3.2.15(D)] indicates that this region possesses the highest B-factors in the whole protein (Muller et al, 2010). Therefore, the failure of assignment for this part might be as a result of the strong chemical exchange caused by its highly dynamic nature. To circumvent this, a series of ${ }^{1} \mathrm{H}^{15} \mathrm{~N}$ HSQC spectra were recorded at different temperatures ( $283 \mathrm{~K}, 293 \mathrm{~K}, 298 \mathrm{~K}, 303 \mathrm{~K}$ and $308 \mathrm{~K}$ ) to test the potential temperature dependence of chemical exchange.

We also employed the sensitivity-improved HSQC sequence that uses a CPMG pulse train during the INEPT stage to reduce the loss of dephasing of spin coherence due to chemical exchange (Mulder et al, 1996). However, despite applying both two approaches above, the state of the spectra was not improved and few newly appeared peaks were found.

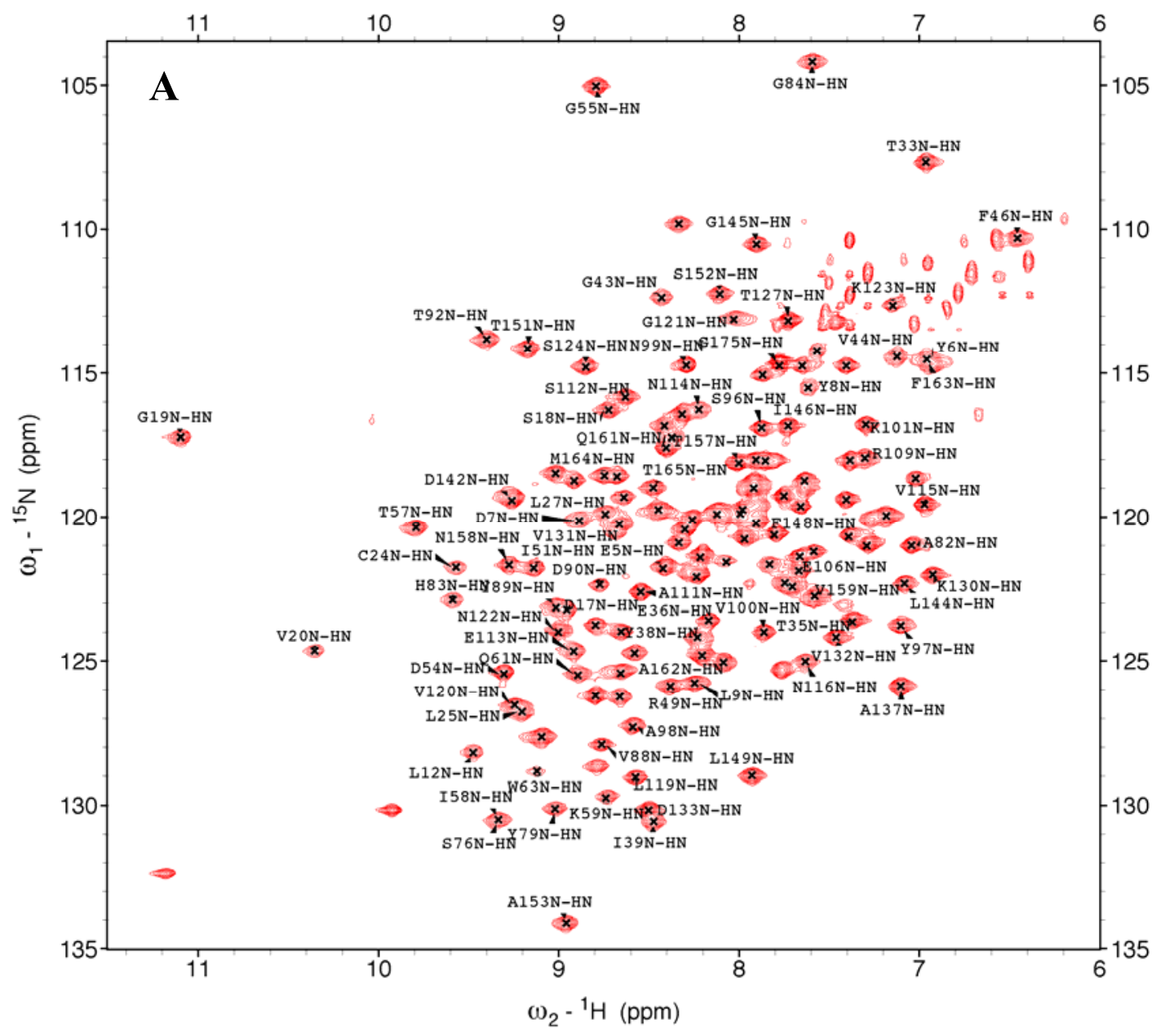




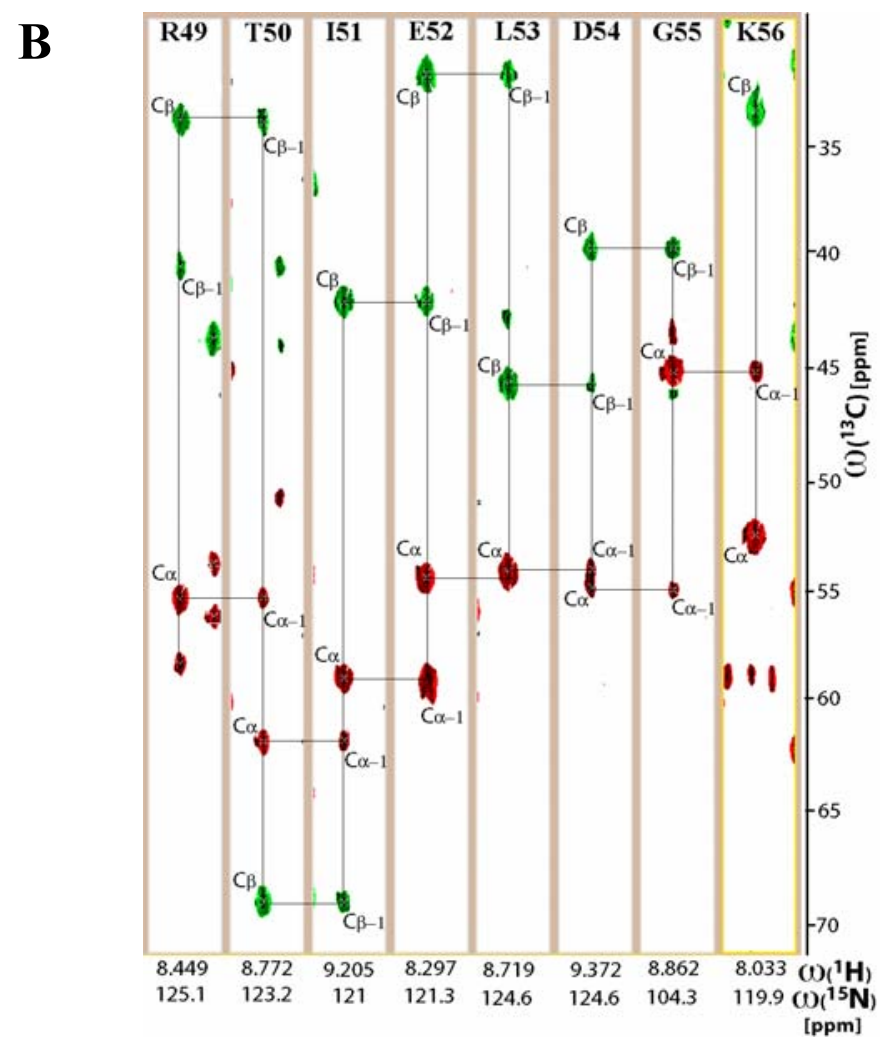

Figure 3.2.14. NMR assignments for Rab1b (1-175). (A) Resonance assignments are indicated with amino acid types and residue numbers in ${ }^{1} \mathrm{H}-{ }^{15} \mathrm{~N}$ TROSY. (B) Strips of the HNCACB spectrum demonstrate the sequential connectivity represented by $\mathrm{C} \alpha / \mathrm{C} \beta$ within the fraction of R49-K56.

To correlate the solution conformation and crystal structure, structural analysis for Rablb was implemented based on the assignment we obtained. Secondary chemical shifts of $\mathrm{C} \alpha$ [Figure 3.2.15(A)] agree with the elements of secondary structure (on the top of the figure) determined by X-ray crystallography (Muller et al, 2010). This indicates that solution conformation measured by NMR is highly consistent with the crystal structure. Furthermore, residual dipolar couplings (RDCs) measured in pf1 bacterial phage shows basic agreement between the crystal structure and secondary chemical shifts [Figure 3.2.15(B), (C)]. However some parts, particularly the conjunction areas between structured and disordered regions, show inconsistence with the other types of structural data, suggesting that these locations adopt different orientations in solution than in crystals. Moreover, the crystal structure of Rablb$\delta \mathrm{C}(\mathrm{GppNHp})(\mathrm{PDB}: 3 \mathrm{NKV})$ is represented with b-factor mapping [Figure 3.2.15(D)]. 
As the core part of the Switch II region, helix $\alpha 2$ displayed in the X-ray structure is relatively short, looser and more distorted than other structured regions. B-factor mapping also points out that this region experiences more intensive thermal fluctuation than other parts, especially when compared with the core region of the fold denoted by the color blue. Hence, the Switch II region is highly dynamic in the protein. This region also provides the structural motif for tight binding of the regulator and effector proteins, i.e. GEF, GAP, GDI, and its conformation is highly sensitive to nucleotides binding (Lee et al, 2009). These features can largely contribute to chemical exchange that induced peak broadening and loss of sensitivity in the NMR spectroscopy. Hence, as described above, this feature accounts for the fact that corresponding resonances of $\alpha 2$ and G3 disappeared in the NMR spectra.
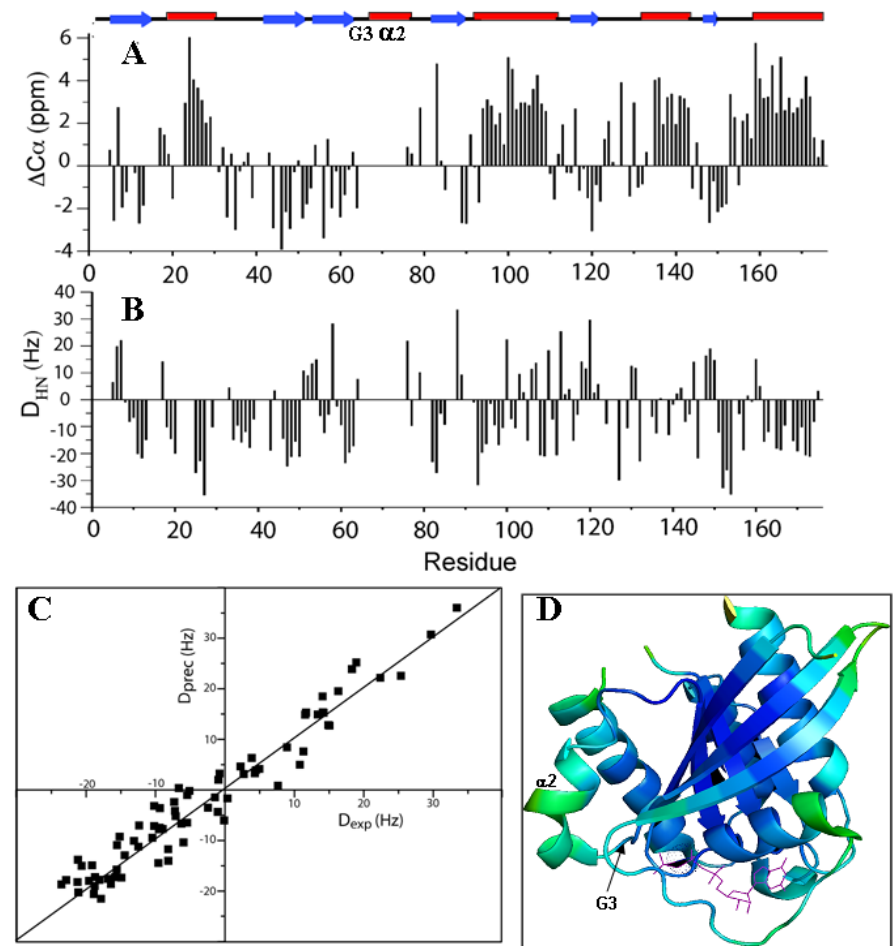

Figure 3.2.15 Structural characterization of $\mathbf{R a b 1 b}-\delta \mathbf{C}$. The secondary chemical shift of $\mathrm{C} \alpha(\mathrm{A})$ and residual dipolar couplings (RDCs) of $\mathrm{NH}$ of $\operatorname{Rab} 1 \mathrm{~b}(1-175)$ (B) are compared with the secondary structural elements determined by X-ray crystallography(The bar on the top: blue arrows indicate $\beta$-strand, red rectangles indicate $\alpha$-Helix and black solid lines indicate the loop). (C) Correlation of experimental RDCs and SVD back-calculated RDCs based on crystal structure. (D) Crystal structure of Rab1b$\delta \mathrm{C}(\mathrm{GppNHp})(\mathrm{PDB}$ code: $3 \mathrm{NKV}$, resolution $1.7 \mathrm{~A})$ is represented with B-factor mapping (green: high, blue: low). The typical Switch II region consisting of $\alpha 2$ helix and G3 loop is denoted. 
Previously, the interaction between monomeric $\alpha \mathrm{S}$ and Rab1b was not observed by NMR titration. The titration experiments were implemented by titrating Rablb material into ${ }^{15} \mathrm{~N}-\alpha \mathrm{S}$ and observing changes in ${ }^{1} \mathrm{H}_{-}{ }^{15} \mathrm{~N}$ resonances of $\alpha \mathrm{S}$. To further confirm this finding, we repeated the titration assay by adding isotopically natural abundance $\alpha \mathrm{S}$ into ${ }^{15} \mathrm{~N}$ - Rab1b- $\delta \mathrm{C}$ and monitoring possible resonance-changes of the Rab1b. Moreover, we also titrated the fibrillar $\alpha \mathrm{S}$ into ${ }^{15} \mathrm{~N}$ Rab $1 b-\delta \mathrm{C}$ to detect potential interactions between $\mathrm{RAb} 1 \mathrm{~b}$ and $\alpha \mathrm{S}$ aggregates since Rab1b can modulate the aggregation of $\alpha \mathrm{S}$.

Figure 3.2.16 shows that there are no significant bindings of Rab1b to $\alpha \mathrm{S}$ monomers and fibrils. For the case of monomeric $\alpha \mathrm{S}$, the region of Rab1b from residue 40 to 60 displays the trend that the chemical shifts were slightly affected by the addition of $\alpha \mathrm{S}$. But in comparison to the rest of the protein, the $\alpha \mathrm{S}$-induced perturbation is not pronounced. This might be due to some nonspecific interactions that partially perturbed this region. Both chemical shift perturbation and intensity plot indicated that no significant interaction took place between Rab1b and $\alpha \mathrm{S}$ fibrils.
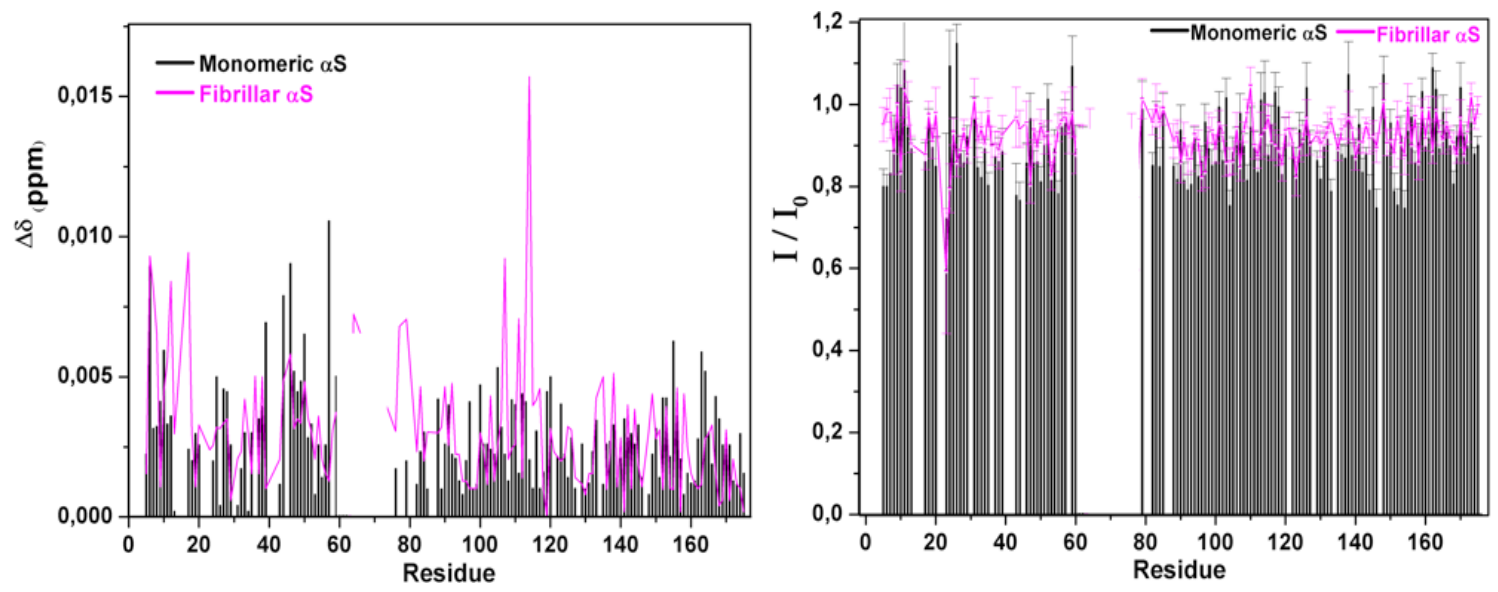

Figure 3.2.16. NMR binding assay for Rab1b and $\alpha \mathrm{S}$. Monomeric and fibrillar $\alpha \mathrm{S}$ were titrated into ${ }^{15} \mathrm{~N}$-enriched Rab1b (1-175) by monitoring the variation of chemical shift (A) and signal intensity (B) of the peaks in the ${ }^{1} \mathrm{H}_{-}{ }^{15} \mathrm{~N}$ HSQC spectrum of Rab1b. The applied Rab1b: $\alpha$ S ratios were 1:7 for monomeric $\alpha \mathrm{S}$ to Rab1b and 1:5 for fibrillar $\alpha \mathrm{S}$ (molar equivalents of monomeric protein).

Additionally, the ${ }^{15} \mathrm{~N}-\mathrm{Rab} 1 \mathrm{~b}-\delta \mathrm{C}$ used for the titration is C-terminally truncated. Thus, the titration did not reflect whether $\alpha \mathrm{S}$ could interact with the C-terminus of Rab1b. Howevert, the Rab1b previously used for titrating into ${ }^{15} \mathrm{~N}-\alpha \mathrm{S}$ is the full-length protein 
and no binding was reflected by the resonances of $\alpha \mathrm{S}$ [Figure 3.2.2 (A)]. Analyzing the sequence of amino acids for $3 \mathrm{Rab}$ proteins also suggests that except Rab8a, the termini of Rab1b and Rab3a do not strongly bind to $\alpha \mathrm{S}$ according to the charge distribution [Figure 3.2.3].

In conclusions, the solution conformation of Rab1b- $\delta \mathrm{C}(\mathrm{GDP})$ is consistent with the crystal structure. Moreover, we did not observe significant interactions between Rab1b- $\delta \mathrm{C}(\mathrm{GDP})$ and $\alpha \mathrm{S}$ monomers and fibrils. However, based on our aggregation assay, Rab1b modulated the aggregation of $\alpha \mathrm{S}$. This suggests that Rab1b likely interacts with aggregation intermediates.

\subsubsection{Assignment and structural analysis of Rab8a}

Rab8a shares highly sequential similarity and identity with Rab1. In parallel to this, the folded parts of these two Rabs also possess high similarity, except their C-terminal loops, which is a conserved feature in Ras GTPases superfamily. Therefore, these factors enable us to apply NMR assignment to Rab8a based on the assignment of Rab1b. However, in comparison to Rab1b, Rab8a is more difficult for sample preparation. First of all, it requires protein chaperones to assist its folding, which is not common in the Rab family. Secondly, Rab8a is insoluble in E. coli expression system and its purification is restricted to C-terminally truncated Rab8a(1-183) due to the structural flexibility of the C-terminus. Through codon-optimization and co-expression with protein the chaperone GroEL/GroES in E. coli, Dr. Amyelt Itzen's lab produced fulllength Rab8a with an improved yield as well as the a reduced insolubility and heterogeneity (Bleimling et al, 2009). However, the expression of the isotopic labeling sample suffered even more difficulties and thereby substantially hampered NMR assignment and assessment for Rab8a. Finally, $100 \mu \mathrm{M}{ }^{15} \mathrm{~N}-$ Rab8a-GDP in volume of $200 \mu \mathrm{L}$ and $300 \mu \mathrm{M}{ }^{13} \mathrm{C},{ }^{15} \mathrm{~N}-\mathrm{Rab} 8 \mathrm{a}-G D P$ in volume of $70 \mu \mathrm{L}$ were prepared. The paucity of the sample largely restricted our NMR studies. However, it still provided us with the opportunity to approach the structural analysis of Rab8a and the mechanistic study on the binding of $\alpha \mathrm{S}$ to Rab8a.

Not surprisingly, in reference to Rablb- $\delta \mathrm{C}(\mathrm{GDP}),{ }^{1} \mathrm{H}-{ }^{15} \mathrm{~N}$ HSQC of Rab8a(GDP) exhibits certain similarity in resonance distribution compared with Rablb due to their 
structural similarity [Figure 3.2.17]. Superposition of the spectra of the two Rabs indicates a few peaks of Rab8a were highly overlapped to the peaks of Rab1b.. Based on the sequence alignment of these two Rab proteins and the assignment of Rablb, these paired peaks should correspond to the conserved residues between the 2 proteins, such as G56 and A154 of Rab8a. These specific residues were helpful for the assignment of Rab8a.

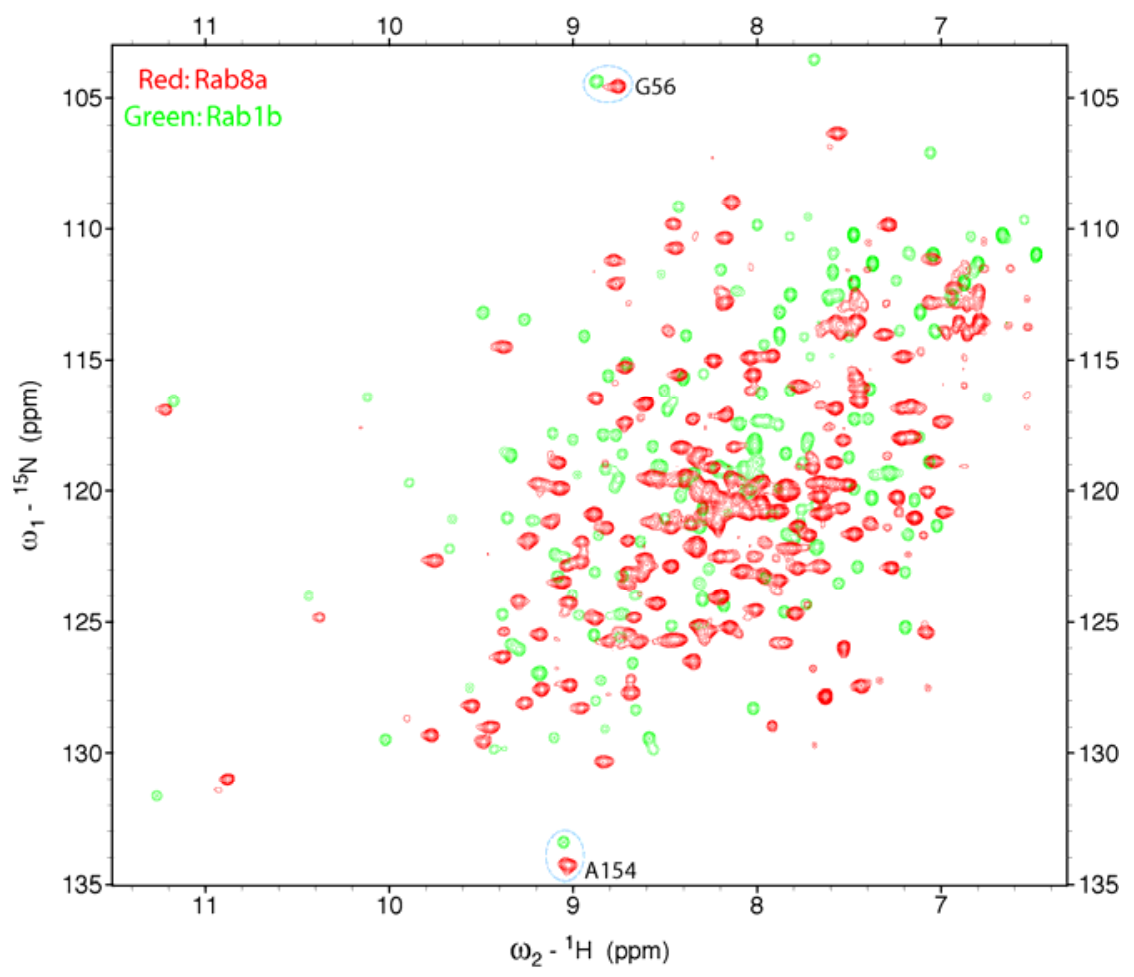

Figure 3.2.17. ${ }^{1} \mathbf{H}-{ }^{15} \mathbf{N}$ HSQC spectrum of Rab8a. HSQC of Rab8a (red) was shown with Rab1b(1-175) (green) as a reference. Both spectra were acquired at $298 \mathrm{~K}$. The conserved residues based on sequence alignment of Rab1b and Rab8a i.e. G56, A154 were labeled.

To correlate with full-length Rab8a, we also acquired natural abundance ${ }^{1} \mathrm{H}^{-15} \mathrm{~N}$ HSQC for the C-terminal peptide of Rab8a which previously identified that it could bind to $\alpha \mathrm{S}$ C-terminus and affect the aggregation of $\alpha \mathrm{S}$. The HSQC of peptide was overlaid with the spectrum of full-length Rab8a, which showed that part of the resonances of the peptide were well overlaid with full-length protein, i.e. I193 and T194, but the rest of resonance were not. This is probably due to peptide adopts different conformation when it remains alone. It is necessary to obtain the assignment of Rab8a 
and implement structural analysis of both the folded part and the C-terminal loop within Rab8a.

Since the sample amount of ${ }^{13} \mathrm{C},{ }^{15} \mathrm{~N}-$ Rab8a was fairly limited, to record the NMR spectra required for assignment of Rab8a, a $1.7 \mathrm{~mm}$ cryogenical probehead was installed on the Bruker 800M spectrometer. 3D TROSY-based HNCA, HNCACB and CBCAcoNH were recorded for assignment. Sample instability caused substantial loss of the sample as the precipitation appeared after long time acquisition and this disabled further spectra aquistion.

Spectra overlaying and sequence alignment identified 3 peaks that belonged to 3 conserved residues between Rab8a and Rab1b, which are Gly20, Gly56, Ala154. The resonance positions of these 3 residues were well-resolved in the HSQC spectrum of Rab8a and were specified in MARS program to enhance the assignment. The cutoffs were optimized as $0.15 \mathrm{ppm}$ for $\mathrm{C}_{\alpha}$ and $0.5 \mathrm{ppm}$ for $\mathrm{C}_{\beta}$ in order to maximally and reliably establish sequential connectivity by MARS.

Finally, 112 out of 209 residues of Rab8a were assigned reliably (appendix II). Secondary chemical shifts of $\mathrm{C} \alpha$ for assigned residues of Rab8a were consistent with secondary structural elements determined by X-ray crystallography for Rab8a- $\delta \mathrm{C}$ [Figure 3.2.19]. The missed assignments were mainly at the Switch II region, Cterminal tail and the short turn between $\beta 2$ and $\beta 3$. The Switch II region comprised of G3 loop and $\alpha 2$ helix (Asp65-Thr77) was also unassigned in Rab1b(1-175) due to its dynamics. Major fragments of the C-terminal loop starting from Asn182 to Lue209 were also largely unassigned. This is because the long loop region of 28 residues leads to the substantial degeneracy of the resonances due to it disordered state. The two proline residues Pro184 and Pro195 also interrupted the establishment of sequential connectivity for this loop region. A few repeats such as Gln197Gln198, Ser201Ser202, Phe203Phe204 and Leu208Leu209 were not readily distinguished very well under the conditions of spectra. Furthermore, the general limitations of the sample such as lower concentration, small amount and high instability also largely retarded the sensitivity and resolution of the NMR spectra. However, based on the half of Rab8a assignment, we still have an opportunity to examine potential bindings of Rab8a to $\alpha \mathrm{S}$. 

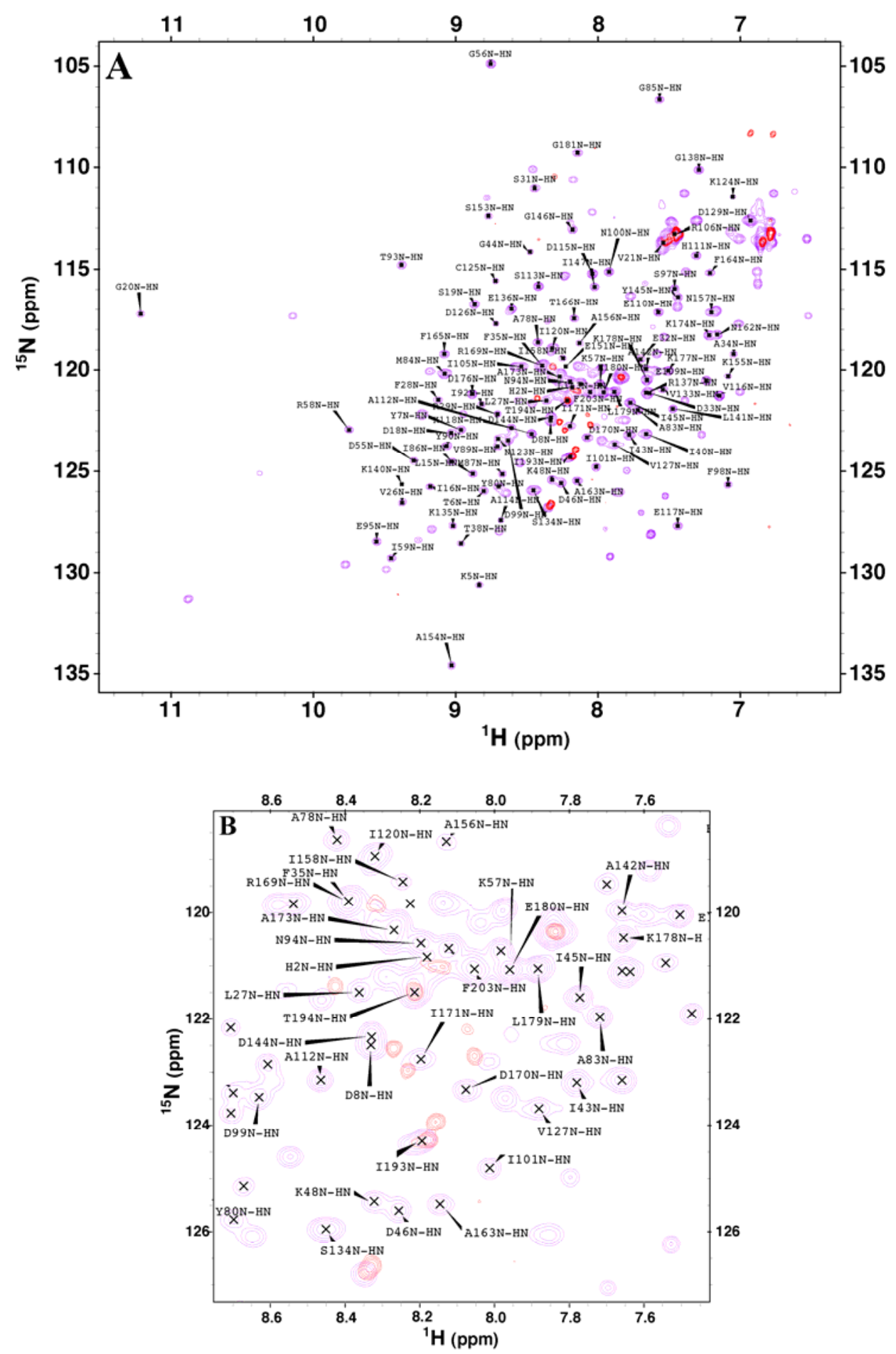

Figure3.2.18. ${ }^{1} \mathrm{H}_{-}{ }^{15} \mathrm{NHSQC}$ spectrum of the C-terminus peptide of Rab8a. (A) ${ }^{1} \mathrm{H}-{ }^{15} \mathrm{~N}$ HSQC for the C-terminus peptide was recorded at natural abundance (red) at 298K and the HSQC for full-length Rab8a recorded at same temperature was shown using the color blue as reference. The region containing most of the peptide resonances is enlarged and displayed in (B) indicating that the fingerprint of two spectrums is partially overlaying. 


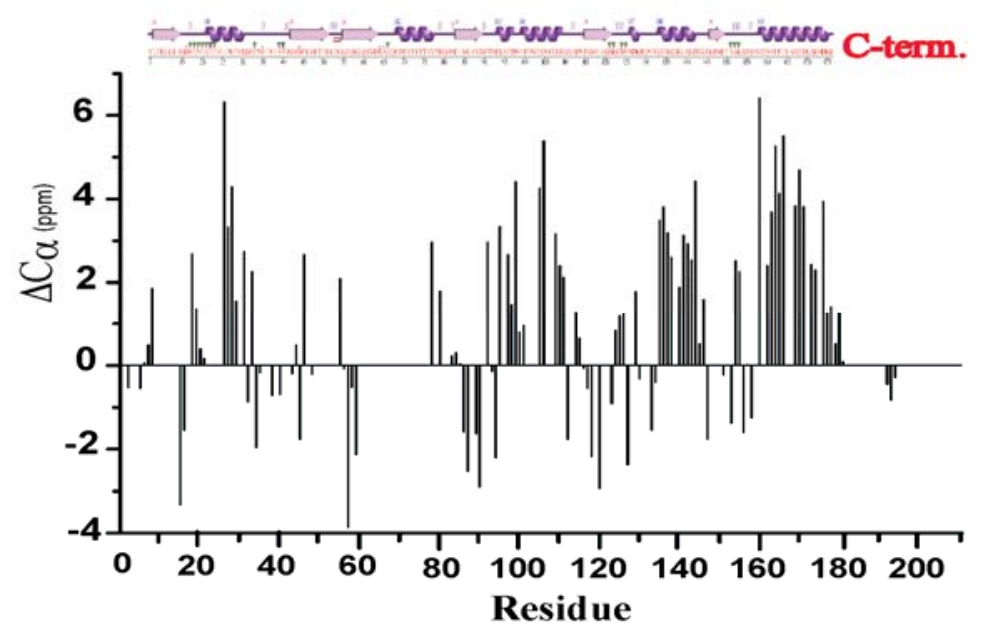

Figure 3.2.19 Secondary chemical shift mapping for Rab8a-GDP. Secondary chemical shifts of C $\alpha$ were mapped aginst residue. The secondary structural elements of Rab8a determined by X-ray crystallography(PDB code: 3QBT) were described on the top of figure (Hou et al, 2011).

\subsubsection{Mapping interaction sites on Rab8a}

As described in the previous sections, both $\operatorname{Rab} 8 \mathrm{a}-\delta \mathrm{C}$ and the C-terminal tail of Rab8a can bind to the $\mathrm{C}$-terminus of $\alpha \mathrm{S}$. Therefore, in order to map the interfaces of Rab8a that enable Rab8a to bind to $\alpha \mathrm{S}$, a series of HSQC spectra of ${ }^{15} \mathrm{~N}-\mathrm{Rab} 8 \mathrm{a}(\mathrm{GDP})$ were recorded with the addition of different amounts of $\alpha \mathrm{S}$ and analyzed based on the attained assignment.

In Figure 3.2.20, three segments of Rab8a(GDP) are distinguished by increasing the added concentration of $\alpha \mathrm{S}$. The first motif is $\mathrm{I}^{43} \mathrm{G}^{44} \mathrm{I}^{45} \mathrm{D}^{46}$ identified by the change of both resonance positions and intensities. The changes of chemical shift were increased as a result of the increased concentration of $\alpha \mathrm{S}$. Interestingly, the resonance intensities of these 4 residues also increased with the continuous addition of $\alpha \mathrm{S}$ and this effect was significantly shown at the molar ratio $\mathrm{Rab} 8 \mathrm{a} / \alpha \mathrm{S}$ of $1: 8$ referencing to the neighbor regions. This suggests that chemical exchange in this region was restricted due to the binding of $\alpha \mathrm{S}$ and thereby the previous broadening of the resonances of these residues was alleviated. Mapping this motif onto the crystal structure of Rab8a(1-178) (Hou et al, 2011), we found that it corresponds to the end of the G2 loop [Figure3.2.20], typically defined as the Switch I region that experiencing drastically structural change during the process of GTP hydrolysis. Switch I region consists of the G2 loop and the beginning of 
the following $\beta 2$ strand, where there are positively charged residues such as K48, R50 that might provide binding potential.

According to the intensity plot [Figure3.2.20. B], the region D55-I59 displayed propensity for increased intensities upon the addition of $\alpha \mathrm{S}$. This fraction is located at the $\beta 3$ strand which is encompassed in the Interswitch region that typically represents the region between the Switch I and Switch II. This Interswitch region normally undergoes allosteric effects caused by the structural regulations of the two Switch regions. Another region from residues A112 to I120 also shows changes in the chemical shifts but not in resonance intensities. This part contains one loop region between the $\alpha 3$ helix and $\beta 5$ strand and the starting fraction of $\beta 5$, which is labeled in pink color in Figure 3.2.21. The composition of amino acids in this region contains D115, E117 and K118 which does not display strongly positive potential and suggests the changes of chemical shifts are likely to be secondary effects of $\alpha \mathrm{S}$ binding.

The C-terminus of Rab8a as a loop region enriched by positively charged residues displayed strong propensity for binding to $\alpha \mathrm{S}$ reflected by both chemical shifts and resonance intensity in Figure 3.2.20 as expected. Missed assignment for the majority of the C-terminus led to substantial loss of binding details at this region. However, M119I120 in the C-terminal loop shows significant chemical shift perturbation since at the ratio of 1:3. These two residues are directly after K117K118 that very likely acts as a binding motif for $\alpha \mathrm{S}$. Moreover, the intensity plot indicates a clear trend of intensity increase starting from A173 until Gly181. In addition, the short fraction K192I193T194 showed intensity increase as well. The increase of intensities observed for these assigned C-terminal residues also indicates that the backbone dynamics were affected by the binding of $\alpha \mathrm{S}$. Thus, in agreement with our previous result from the Rab8a C-terminal peptide, the C-terminus of Rab8a provides a another binding motif for $\alpha \mathrm{S}$. 
Titration of $\alpha$ S into ${ }^{15} \mathrm{~N}-$ Rab8a(GDP)
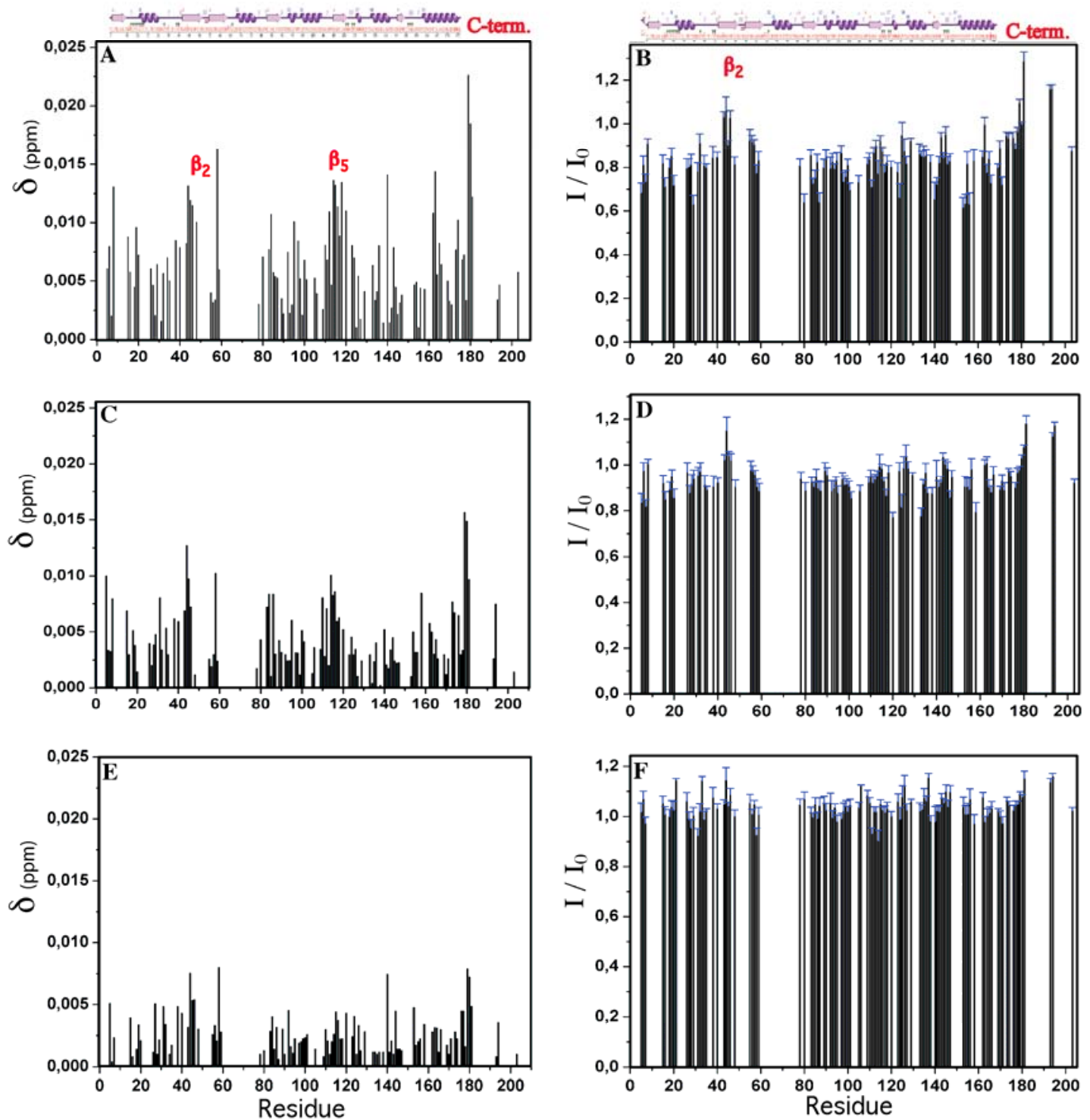

Figure3.2.20 $\alpha \mathrm{S}$ binds to Rab8a-GDP revealed by NMR titration. Changes in chemical shift and intensity of resonances of ${ }^{1} \mathrm{H}^{-15} \mathrm{~N}$ HSQC spectra of Rab8a-GDP were resulted from the addition of $\alpha \mathrm{S}$ with 1:1, 1:3 and 1:8 molar ratio of Rab8a: $\alpha$ S. The changes of chemical shifts and intensity are reflected in (A) and (B) for the ratio of 1:8, (C) and (D) for the ratio of 1:3, (E) and (F) for 1:1. Error bars for intensity plots were estimated based on signal to noise. 

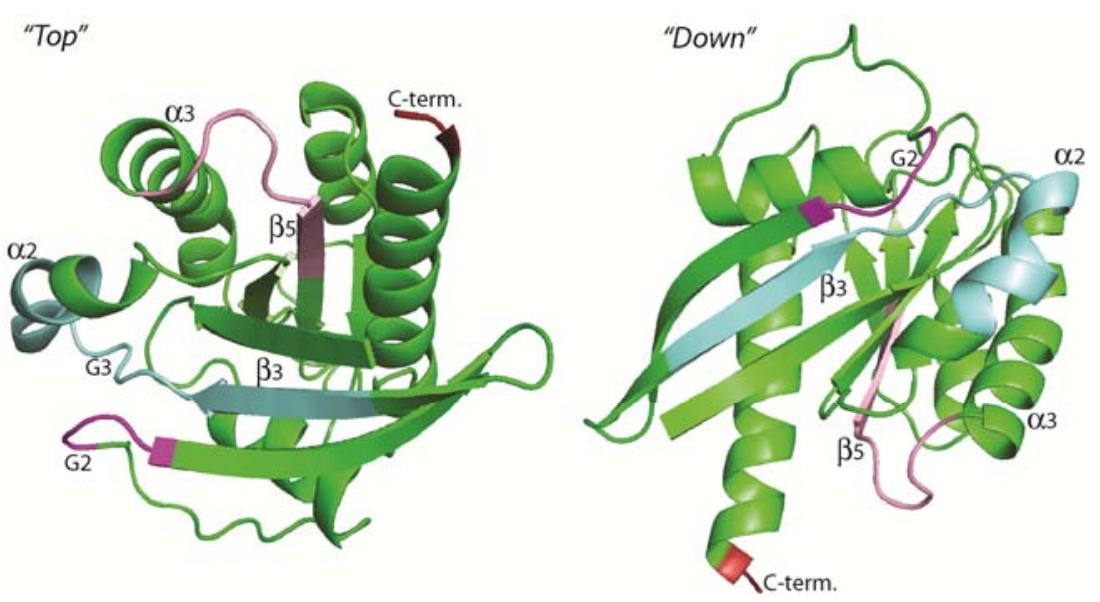

Figure 3.2.21. Mapping interactions between Rab8a-GDP and $\alpha \mathrm{S}$. The binding of $\alpha \mathrm{S}$ to Rab8a was recognized by the NMR titration assay and mappedonto the crystal structure of Rab8a (9-178)-GppNHp (PDB: 3QBT). Both "top" (left) and "down" (right) views are displayed. The binding motif of $\mathrm{I}^{43} \mathrm{G}^{44} \mathrm{I}^{45} \mathrm{D}^{46}$ which is part of G2 loop is labeled in magenta. Another motif of residues A112 to I120 is labeled in pink color. The unassigned Switch II region is labeled in light blue and the starting of Cterminal loop is labeled in red. According the nomenclature for secondary structure elements of RasGTPases, loops $(\mathrm{G} 2, \mathrm{G} 3)$, helixes $(\alpha 2, \alpha 3), \beta$-strands $(\beta 3, \beta 5)$ are selectively noticed.

In conclusion, by using NMR titrations assays, we defined the interactions between Rab8a and monomeric $\alpha \mathrm{S}$. The binding is reflected in the NMR spectra of both Rab8a and $\alpha \mathrm{S}$. For $\alpha \mathrm{S}$, the binding of Rab8a occurs at its highly negatively charged Cterminus. Rab8a is a protein that is enriched by positively charged residues at its Cterminus and N-terminally folded parts as well. This feature of charge distribution enables Rab8a to offer multiple sites for $\alpha \mathrm{S}$ binding.

Both GDP- and GppNHp-bound Rab8a can bind to $\alpha$ S but Rab8a(GDP) displays stronger binding affinity to $\alpha \mathrm{S}$ than Rab8a(GppNHp). Moreover, the structural data of $\alpha \mathrm{S}$ binding to Rab8a indicates that the binding likely reduces the chemical exchange around the major binding sites of Rab8a, i.e. the Switch I region and the C-terminal loop. Unlike Rab8a, Rab1b and Rab3a do not bind to monomeric $\alpha \mathrm{S}$, but they enhance the aggregation of $\alpha \mathrm{S}$ in a very similar manner, indicating that they potentially interact with $\alpha \mathrm{S}$ aggregates and facilitate fibrillization. 


\subsection{Structural characterization of alpha-Synuclein aggregates}

The structural study of $\alpha \mathrm{S}$ aggregates is important to understand the pathological consequences of $\alpha \mathrm{S}$. Fibrils of $\alpha \mathrm{S}$ are recognized as a major component of Lewy bodies, the neuronal inclusion viewed as the hallmark of Parkinson's disease(PD) (Spillantini et al, 1997). And $\alpha \mathrm{S}$ oligomers, the aggregation intermediates, were proposed as major toxic species responsible for neuronal degeneration of PD. Until now, fibril structure of $w t-\alpha \mathrm{S}$ has been well-characterized by EPR (Chen et al, 2007), mass spectrometry (Del Mar et al, 2005), solid-state NMR (ssNMR) (Comellas et al, 2011; Heise et al, 2008), and combination of solution NMR and ssNMR (Vilar et al, 2008). On the other hand, oligomeric species as the intermediates of $\alpha \mathrm{S}$ aggregation are rarely characterized by structural methods at atomic resolution due to their instability and heterogeneity.

As described in sections 1.2, $\alpha \mathrm{S}$ fibrils still play an important role in PD pathology; and three disease-related mutants present different cellular physiologies and structural features. However, previous structural studies on fibrils of E46K and A53T $\alpha \mathrm{S}$ by ssNMR still didn't achieve consensus regarding structural perturbation of the mutations to $\beta$-sheets enriched fibril core, particularly for E46K (Comellas et al, 2011; Heise et al, 2008). Moreover, a conserved solvent-protected core in amyloid fibrils of $w t$ and A30P aS has been observed (Cho et al, 2011). It is also unknown whether a conserved core also exists in amyloid fibrils of E46K and A53T $\alpha \mathrm{S}$ ? To address these questions, we performed H/D exchange by solution NMR on the fibrils of these two mutants.

Most of low resolution data suggest that oligomers of $\alpha \mathrm{S}$ mainly adopt $\beta$-sheet conformation similar to the fibrils. Thus, with the aid of the compounds that specifically maintain $\alpha \mathrm{S}$ at oligomeric stage with certain size, we performed the optimized NMR H/D exchange method to tentatively detect the potential hydrophobic protections inside the oligomers.

\subsubsection{H/D exchange for fibrils of E46K and A53T $\alpha$-Synuclein mutants}

Mutations of A30P, E46K and A53T are associated with familial forms of PD. Structurally, these 3 mutants were reported to perturb intra-molecular long-range 
interactions between $\mathrm{N}$ - and $\mathrm{C}$ termini of $\alpha \mathrm{S}$ and thereby alter its aggregation behavior (Bertoncini et al, 2005a). The A30P mutation slows $\alpha \mathrm{S}$ fibrillization and produces more soluble oligomeric intermediates. However, it does not change the arrangement of $\beta$ sheets in core region of the fibrils (Lemkau et al, 2012). Compared with A30P, the E46K and A53T mutations are closer to the NAC region centrally for fibril formation. Both $\mathrm{E} 46 \mathrm{~K}$ and $\mathrm{A} 53 \mathrm{~T}$ promote the fibril formation and display faster kinetics than $w t$ $\alpha \mathrm{S}$ (Fredenburg et al, 2007; Greenbaum et al, 2005). Moreover, by morphological comparison, ultrastructural diversity of amyloid fibrils was observed amongst the mature fibrils of $w t-\alpha \mathrm{S}$ and its 3 mutants (van Raaij et al, 2006). These findings together suggest that the familial mutations of $\alpha \mathrm{S}$ modify both fibrillization kinetics and assembly of protofilaments. It's thus intriguing to investigate whether the fibrils of E46K and A53T adopt a similar conformation at their fibril core region respecting to the fibrils of $w t$ and $\mathrm{A} 30 \mathrm{P} \alpha \mathrm{S}$. Furthermore, it is interesting to assess how modifications of micro-environment of amino acids impact on fibrillization kinetics and ultrastructural assembling of fibrils.

The monomers of E46K and A53T $\alpha \mathrm{S}$ were incubated respectively under the aggregation condition at $37^{\circ} \mathrm{C}$ with constant stirring. Fibrils were harvested after 2 weeks. The formation of fibrils was characterized by ThT fluorescence and electron microscopy. ThT fluorescence of E46K $\alpha \mathrm{S}$ aggregation after 2 weeks was 3 fold higher than A53T $\alpha \mathrm{S}$. Comparison of EM images illustrated that the fibrils of A53T $\alpha \mathrm{S}$ adopted a typical morphology of $\alpha \mathrm{S}$ fibrils, but the fibrils of E46K $\alpha \mathrm{S}$ were relatively shorter, more branched and heterogeneous [Figure 3.3.1]. Therefore, the aggregation kinetics and morphological analysis indicates that these two $\alpha \mathrm{S}$ mutants differ in manner of fibrillization and particularly in assembly of the protofilaments.

After two weeks of aggregation, the fibrils of E46K and A53T $\alpha \mathrm{S}$ were collected by ultracentrifugation with $60 \mathrm{k}$ rmp for 2 hours at $4^{\circ} \mathrm{C}$. The pellet of fibrils was then resuspend into distilled $\mathrm{H}_{2} \mathrm{O}$ and ultracentrifuged again for removal of soluble components attached to the fibrils. At the end, the pellets of fibrils were suspended into the exchange buffer containing $99.9 \% \mathrm{D}_{2} \mathrm{O}, 200 \mathrm{mM} \mathrm{NaCl}$ and $0.1 \%$ formic acid $(\mathrm{pD}$ 4.0). The system was set on a rotator for 1 week in the cold room where the temperature was maintained at $4^{\circ} \mathrm{C}$. 

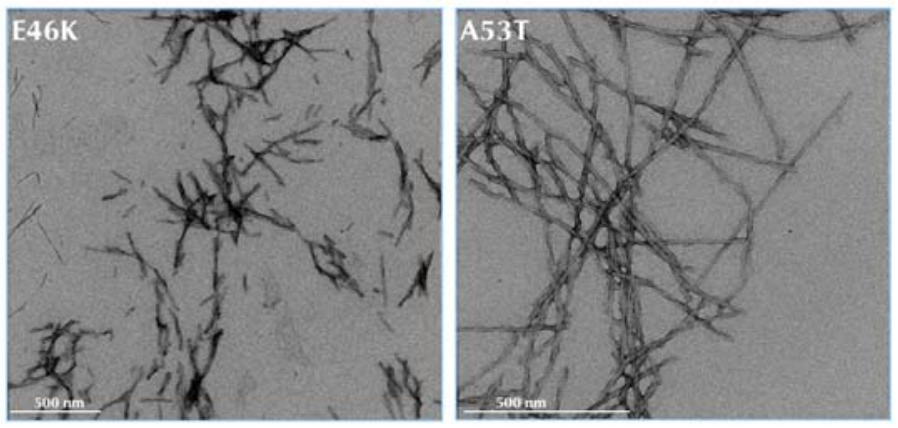

Figure 3.3.1. Electronic microscopy for fibrils of E46K and A53T $\alpha \mathrm{S}$. The fibrils were collected after 2 weeks under the aggregation condition and used for the following H/D exchange.

The fibrils after 1 week of H/D exchange were collected and washed 2 times by ultracentrifugation. The prepared fibrils were dissolved to monomers in $50 \% \mathrm{D}_{2} \mathrm{O}$ solution with $2 \mathrm{M} \mathrm{GuSCN}, 0.4 \%$ formic acid (pD 2.4). Since the fibril pellets were dissolved, the monomers encoding the structural information of fibrils in solution were quickly transferred into the NMR spectrometer to start recording spectra. All the NMR parameters of acquisition were calibrated prior to fibril dissolution using a reference sample prepared in identical solution conditions. After a 12 minutes deadtime for sample tuning in spectrometer, a series of ${ }^{1} \mathrm{H}^{15}{ }^{15} \mathrm{HSQC}$ spectra with band-selective excitation (BEST-HSQC) were recorded with interval of 25 minutes for each spectrum.

Comparing with the solution used for forward-exchange described previously, the concentration of $\mathrm{D}_{2} \mathrm{O}$ was reduced from $100 \%$ to $50 \%$ in the dissolving solution of GuSCN. This possesses the advantages that the region previously protected or exposed in the $100 \% \mathrm{D}_{2} \mathrm{O}$ environment can be distinguished by observing the decreasing or increasing trends of intensity in $50 \% \mathrm{D}_{2} \mathrm{O}$, as a process of back-exchange. In addition, the $\mathrm{pH}$ was set at 2.4 and the spectra were acquired under $5^{\circ} \mathrm{C}$, which maintained the proton/deuterium exchange rate at a minimum level and enabled us to follow the kinetics of back-exchange with a better time resolution under given spectroscopic conditions. 


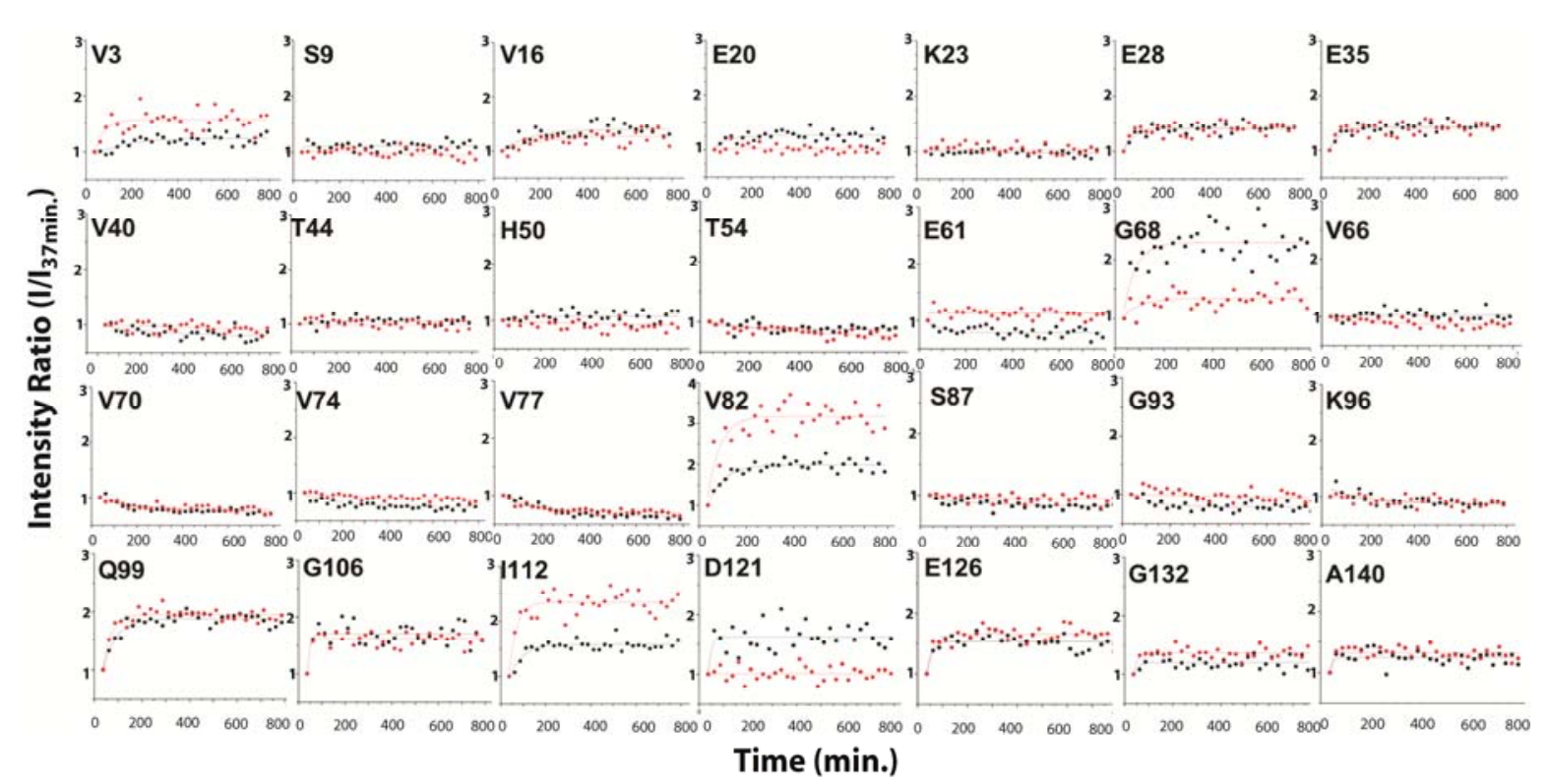

Figure 3.3.2. Intensity plots of HSQC spectra for back-exchange. The residue-specific resonance intensities were mapped as a function of dissolution time starteing from the time that fibrils were suspended into the GuSCN/D $\mathrm{D}_{2} \mathrm{O}$ solution (black:E46K, red:A53T). Each back-exchange profile was fit with a single exponential function curve.

In Figure 3.3.2, residues from different regions of $\alpha \mathrm{S}$ respond differently during the back-exchange process and a few residues of the two mutants experience different kinetics. Most of the selected residues at the N-terminus display an exponential increase, such as V3, V16, E20, E28, E35, suggesting that these residues are exposed to $\mathrm{D}_{2} \mathrm{O}$ during the forward exchange. Distinctively, residues from 37 to 97 majorly adopt a relatively flat or decreasing pattern in the intensity plot, denoting that the residues from this segment were buried inside the structured elements of mature fibrils and the amide protons were protected against exogenous deuterium by forming hydrogen bonds inside the fibrils. A few exceptions, like G68 and E82, implicates they might locate at the loop region between $\beta$-sheets. For the C-terminal residues starting from 98, both mutants showed an obvious increasing pattern in their intensity due to the less protection of the C-terminus than that of fibril core during forward exchange.

To quantitatively map the extent of protection and depict structure of fibril core, according to the back-exchange curve, we back-calculated the initial hydrogen content and the corresponding level of deuterium incorporation as two alternative representations for residue-specific protection extent of the fibrils against H/D exchange during forward-exchange [Figure3.3.3 (A) and (B)]. The patterns of both figures 
consistently and clearly define the boundaries of fibril core at residue $37 / 38$ and 97/98. According to the protection pattern, this core region is conserved in fibrils of both E46K, A53T $\alpha \mathrm{S}$. Out of the core region, C-terminal residues 98-140 were most strongly affected by the exchange and obtained averagely highest extent of deuterium incorporation. N-terminal domain also possessed significantly less protection than fibril core but relatively less pronounced than the C-terminus, indicating that there were likely residual structures presenting in N-terminal (Gath et al, 2012).

Based on the protection patterns inside fibril core, the putative $\beta$-sheets $1,2,3,4$, $5 \mathrm{a}$ and $5 \mathrm{~b}$ were depicted on the top of the figures by identifying the putative loops between two consecutive $\beta$-sheets by recognizing the residues with less protection. This structural arrangement is in agreement with the previously defined fibril structures of $w t-\alpha \mathrm{S}$ and A30P $\alpha \mathrm{S}$ by H/D exchange coupled with HR-MARS (Cho et al, 2011), where the loop residues could be recognized by HR-MARS due to their flexibility.

Interestingly, the basic arrangement of fibril core remains conserved in both mutants. Slight difference is shown that the boundary residues were shifted from V37/K97 for E46K $\alpha \mathrm{S}$ to L38/K96 for A53T $\alpha \mathrm{S}$. Accordingly, the first and last two putative $\beta$-sheets were shifted by one residue for the fibrils of A53T $\alpha \mathrm{S}$ compared with the fibrils of E46K $\alpha \mathrm{S}$. But, generally, the structural patterns of the fibrils of these two $\alpha \mathrm{S}$ mutants demonstrate that the mutations didn't drastically alter the conformation of fibrils core. The difference shown in EM pictures for these two mutants might account for that polymorphism of amyloid fibrils and association fashion of protofilaments was modified by mutation. At position 46, the mutation from Glu to Lys can reverse the charge state of side chain and potently affect the local structure and ambient regions. However, by protection pattern we notice that this position locates likely at the loop region between first and second $\beta$-sheets of fibril core and thus this mutation should not strongly change the arrangement of core. Moreover, no significant changes appeared at neighboring region of T53 by comparing with E46K fibrils. 


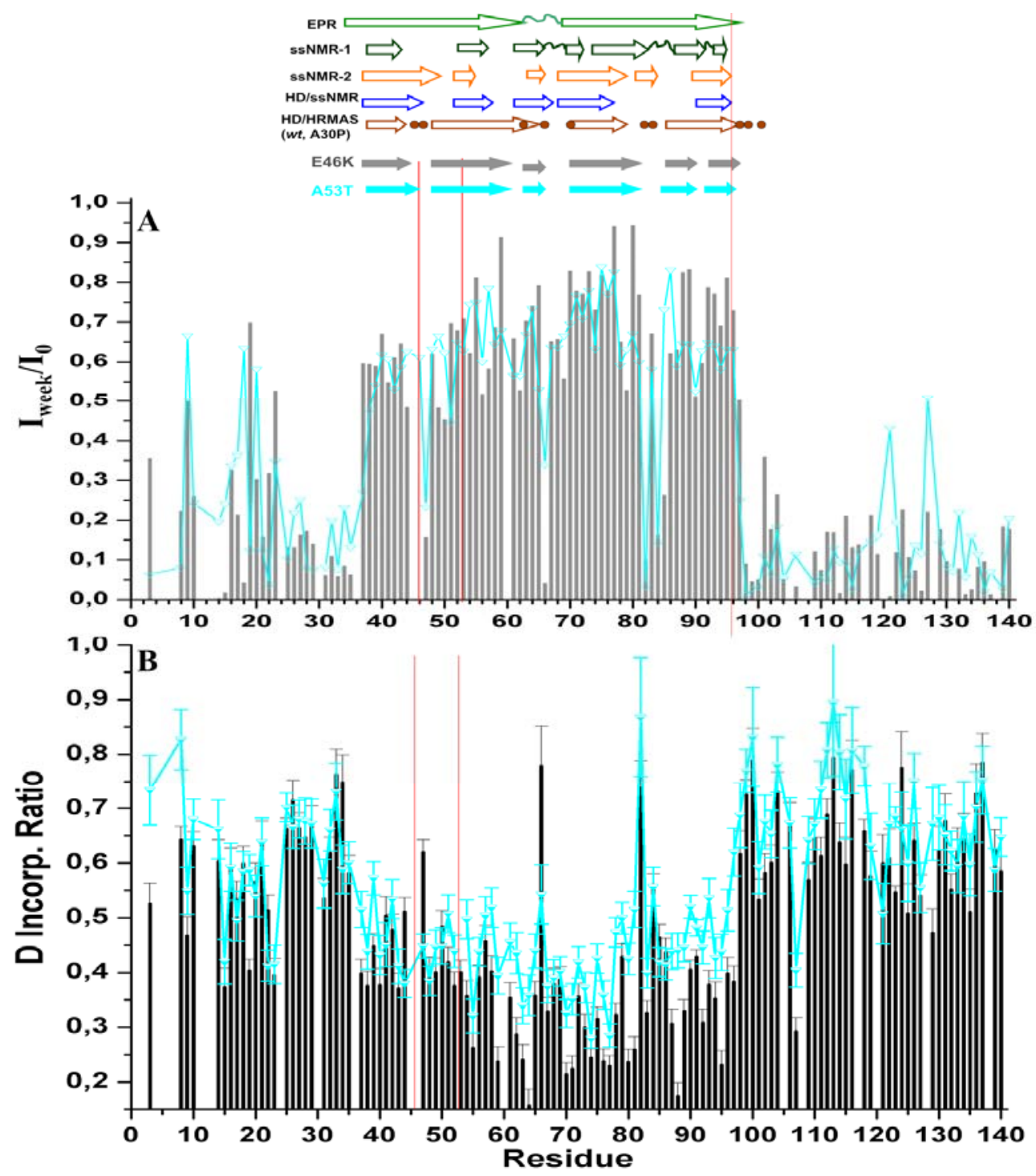

Figure 3.3.3. Characterization of protection extent against H/D exchange for fibrils of E46K, A53T

$\alpha \mathrm{S}$. The mutation positions in protein sequence were labeled by red lines. The back-exchange profile was fit with single exponential function and extrapolated the initial intensity representing the hydrogen content at the starting point of back-exchange (A). Correspondingly, in (B), the original level that deuterium incorporated into fibrils after 2 weeks H/D exchange was back-calculated from back-exchange profile and taken into account that after completion of the back-exchange the protonation level was $47 \%$ (with calibration due to extra protons introduced by GuSCN). Error bars were calculated on the basis of the signal-to-noise ratio in the last and first HSQC spectrum. At the top of figure, the secondary structural elements of $\alpha \mathrm{S}$ fibrils determined previously are described as arrows for $\beta$-sheets and as a curve for linker. (EPR(Chen et al, 2007); solid state NMR (ssNMR-1) (Heise et al, 2005); solid state NMR (ssNMR-2) (Comellas et al, 2011); H/D exchange and solid state NMR(Vilar et al, 2008); H/D exchange and HRMAS (Cho et al, 2011), filled circle represents the flexible regions observed by HR-MAS.) 
In summary, the mutations of E46K and A53T don't induce dramatical change of the solvent protection pattern of fibril core, suggesting a conserved core region in fibrils of these two mutants. And this conserved core is highly similar to fibrils of $w t-\alpha \mathrm{S}$ and A30P $\alpha \mathrm{S}$. Therefore, our results suggest that a highly conserved structure embedding in fibril core of $w t$ - $\alpha \mathrm{S}$ and its 3 familial mutants.

\subsubsection{Probing conformation of the compounds-stabilized oligomers of $\alpha-$ Synuclein}

Several lines of evidences attribute neuron toxicity of $\alpha \mathrm{S}$ to its oligomeric states (Caughey \& Lansbury, 2003; Cookson, 2009; Cookson \& van der Brug, 2008; Lashuel et al, 2002a; Lashuel et al, 2002b; Volles \& Lansbury, 2003; Winklhofer et al, 2008). Thus it's of central interests to study physiological and biophysical features of $\alpha \mathrm{S}$ oligomers. However, as aggregation intermediates, continuum distribution of molecular weight, highly structural heterogeneity and weak stability of the oligomers pose hindrances for us to acquire structural information of $\alpha \mathrm{S}$ oligomers with high resolution and accuracy, which largely hampers us to understand in-depth mechanism of its neuronal toxicity.

A series of organic compounds have been successfully screened for developing potential therapeutic agents of PD by Sergey Ryazanov and Andrei Leonov in our department. These compounds can block fibrillization of $\alpha \mathrm{S}$ by stabilizing the oligomers within a certain size range (hydrodynamic radii of $\sim 100 \mathrm{~nm}$ ) and significantly reduce cellular toxicity of $\alpha \mathrm{S}$ in serveral animal models. Biophysical characterizations demonstrate that these compounds effectively convert the $\alpha \mathrm{S}$ oligomers from onpathway to off-pathway, from larger size to the smaller size with pronounced stability. Solution NMR investigation points out that these compounds do not bind to $\alpha \mathrm{S}$ monomers, but preferentially interact with $\alpha \mathrm{S}$ oligomers. Furthermore, recent research defined that there was one conversion from smaller oligomers of $\alpha \mathrm{S}$ to the larger oligomers, which the $\alpha \mathrm{S}$ oligomers with larger size were putatively more toxical than the smaller ones in cell tests (Cremades et al, 2012).

Therefore, the better stability and promising clinical application stimulates us to access high resolution structural information for these compounds-stabilized $\alpha \mathrm{S}$ 
oligomers. The H/D exchange method was adopted. Based on experience applied for $\alpha \mathrm{S}$ fibrils, we specifically optimized the method for studying conformation of the compounds-stabilized oligomers.

The oligomer sample for H/D exchange was prepared by the standard aggregation procedures with the addition of compounds before aggregation started with ratio that $100 \mu \mathrm{M} \alpha \mathrm{S} / 100 \mu \mathrm{M}$ sery166a or $70 \mu \mathrm{M} \alpha \mathrm{S} / 100 \mu \mathrm{M}$ anle138c. Both sery166a and anle138c are names of the compounds that stabilize $\alpha \mathrm{S}$ oligomers [Patent No.: WO 2010/000372 A2]. The aggregation of $\alpha \mathrm{S}$ was maintained 3 4 days that maximal amount of oligomers was expected. At the end, sample was monitored by Atomic force microscopy (AFM), Dynamic light scattering (DLS) and Thiol-flavinT fluorescence (ThT) [Figure 3.3.4]. Fluorescence shows that no ThT-positive fibrils were formed under the aggregation condition up to 3 days. DLS measurement identified that the sizes of these oliomgers mainly distributed around $100 \mathrm{~nm}$ (hydrodynamic radii) and AFM characterized the oligomers with annular shapes distinct to the classical morphology of $\alpha \mathrm{S}$ fibrils. To remove filaments probably existing in the solution, the samples were centrifuged with $5 \mathrm{~K}$ rpm for 15 minutes prior to $\mathrm{H} / \mathrm{D}$ exchange.

A

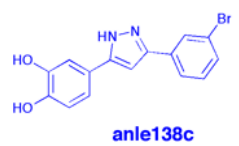

C

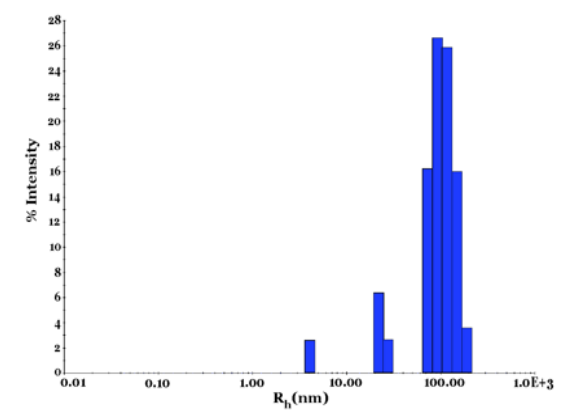

B

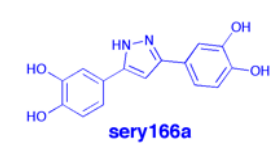

D

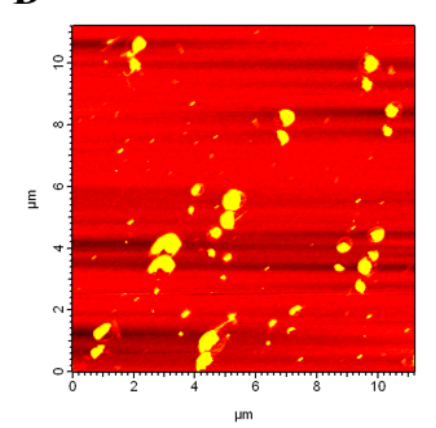

Figure 3.3.4. Biophysical characterization for the compounds-stabilized $\alpha \mathrm{S}$ oligomers. Molecular structures of anle138c and sery166a are shown (A). ThT fluorescence (triplicate measurements) indicates that no fibrils formation after 3 days aggregation with anle138c (A). The oligomers were characterized by DLS with hydrodynamic radii around 100nm (B) and Atomic force microscopy with annular shape (C). 
A primary consideration differing to the case of $\alpha \mathrm{S}$ fibrils is that the oligomeric species of $\alpha \mathrm{S}$ are soluble and hard to be separated from mostly populated $\alpha \mathrm{S}$ monomers by the means like ultracentrifuge. Thus, for the process of forward H/D exchange, we directly added $\mathrm{D}_{2} \mathrm{O}$ into the oligomer solution to reach the $\mathrm{D}_{2} \mathrm{O}$ ratio not less than $50 \%$. Then the whole system was kept in cold room of $4^{\circ} \mathrm{C}$ for forward exchange of 10 minutes. The following procedure including oligomer dissociation by GuSCN, quick transfer and tuning of spectrometer was mostly same as the H/D exchange for fibrils.

Two factors we took into account were: 1) unlike fibril sample, the deuterated oligomers were difficult to be further isolated from monomers; 2) the central part of oligomers was assumed to be solvent exchange-resistant due to hydrogen bonds and hydrophobicity. Therefore, after the forward exchange GuSCN was directly added into the oligomer solution to dissociate oligomers. After the dissociation by GuSCN, there should be different responses for the fraction inside central part of the oligomers and for the fraction that is out of hydrophobic center and highly exposural to solvent. Moreover, the original $\alpha \mathrm{S}$ concentration used for aggregation was $100 \mu \mathrm{M}$ and reduced to less than $50 \mu \mathrm{M}$ after the addition of $\mathrm{D}_{2} \mathrm{O}$ and GuSCN. Thus longer experimental time for each HSQC spectrum was used to compensate signal to noise.

Under the condition described above, the H/D exchange profile after the addition of GuSCN is shown in Figure 3.3.5. Unlike profile of the fibril exchange, 6 selected residues from different parts of $\alpha \mathrm{S}$ didn't display region-specific kinetics. In the case of fibril samples, the residues like A53, I88 were highly associated with the hydrophobic core of $\alpha \mathrm{S}$ fibrils. But their H/D exchange profile of the oligomer sample reflected by peak intensities didn't tend to drop as expected. Moreover, most of residues showed a relatively plateau profile, suggesting that they have been saturated by solvents during the exchange process before starting dissociation. Only a few residues like Y133 tended to decrease, but this might be a result of bulkily side chain of residue which retarded solvent accessibility. Additionally, we observed strong average uncertainty represented by error bars. This was due to low concentration of $\alpha \mathrm{S}$ as the solution was diluted by the addition of large extra amount of $\mathrm{D}_{2} \mathrm{O}$. 

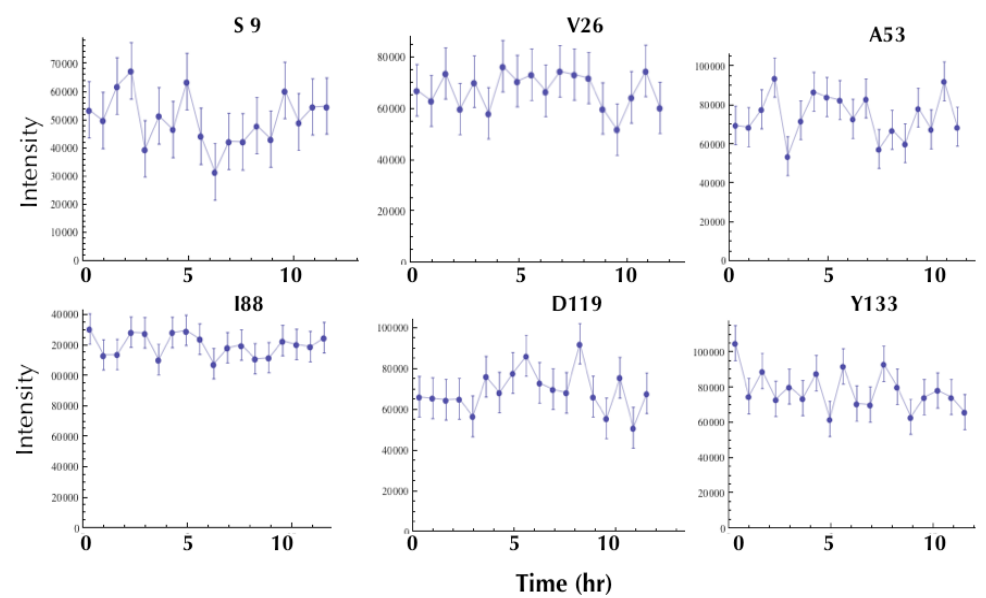

Figure 3.3.5. H/D exchange profile of the compound-stabilized (anle138c) $\alpha \mathrm{S}$ oligomers. The curves represent the residue-specific change of peak intensities after the GusSCN dissociation. 6 residues from $\mathrm{N}$-terminal, NAC and C-terminal are shown. The unit of $\mathrm{Y}$ axis is arbitrary.

Since exchange profiles of most residues were nearly plateau, it implicated that probably both the fractions outside and inside of the presumably hydrophobic center of oligomers already was saturated by solvent during the first exchange period. We considered that hydrophobic interactions and compactness of the oligomers should not be as strong as inside fibril core and the possibility to form hydrogen bonds should also be less than the fibrils. Therefore, it's necessary to improve the exchange conditions to circumvent these drawbacks. $\backslash$

Firstly, we raised the $\mathrm{D}_{2} \mathrm{O}$ ratio from $50 \%$ to $70 \%$ gradually for amplifying the potential difference of exchange behaviors between the central part and the outer part of the oligomers during the first period of the exchange. Secondly, considering that the oligomers might adopt a relatively loose conformation and thus the solvent could approach the assumed core region more easily than the case of fibrils, the exchange time was shorten from 10 minutes to 5 minutes. Under such condition, more solvent-exposed residues were expected to finish the deuterium to proton exchange within 5 minutes but the central part was not. Furthermoe, to improve sensitivity of NMR spectra, we used the membrane with specific molecular weight cut-off filter (i.e. $10 \mathrm{kDa}$ ) to concentrate the sample prior to the exchange condition. 

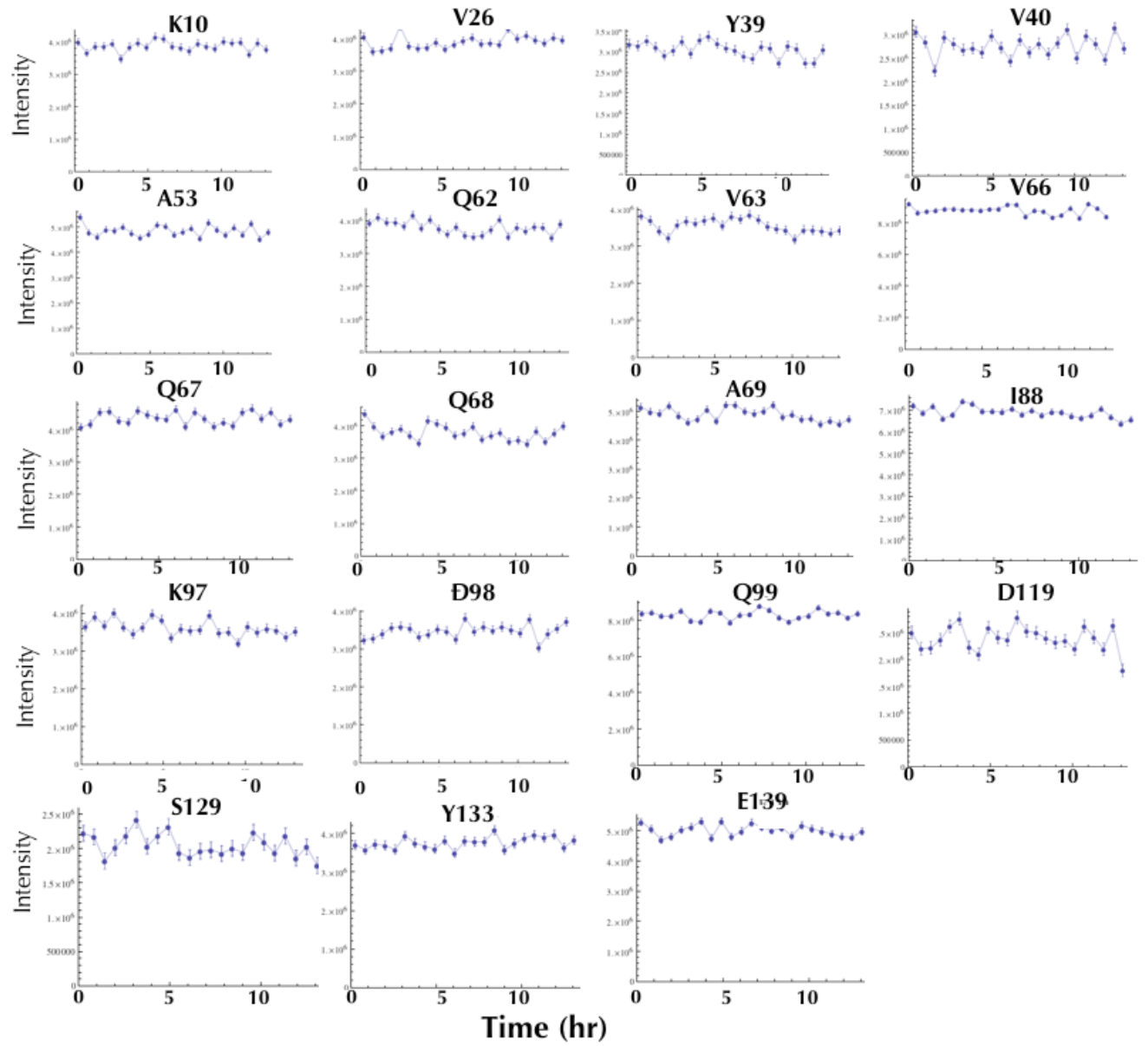

Figure 3.3.6. Optimized H/D exchange for the compound(anle138c)-stabilized oligomers. The peak residue-specific intensities were drawn as the function of exchange time after adding GuSCN. The unit of $\mathrm{Y}$-axis is arbitrary.

Compared with the previous trials, under this improved condition, overall sensitivity reflected by the error bars was enhanced and the exchange curves of residues display slightly distinguishable patterns [Figure3.3.6]. Most of residues from N-terminal part like K10 and V26 tended to adopt plateau trend during 13 hours exchange time. Residue $\mathrm{Y} 39$, at the boundary region of $\alpha \mathrm{S}$ fibrils defined by previous fibril exchange, showed slightly decreasing trend in the $\alpha \mathrm{S}$ oligomeric sample. The residues like A53, V63, Q68, A69, I88 also exhibited the decreasing trend during the exchange. These residues were previously identified in $\beta$-sheets inside of fibril core. Other residues previously in the fibril core region like Q62, V66, Q67 showed fairly weak decrease or even slight increase. Interestingly, these residues located in the loops between two $\beta$ sheets of fibril core found by previous fibril exchange experiment. K97 should be the 
residue at another boundary of the fibril core. The oligomeric sample showed that this residue display slight decrease pattern contrary to its following residues D98 and Q99 which were experiencing increase and plateau respectively but no decrease. Thus, these observations implicate that potential correlation of protection pattern against the solvents between the compound(anle138c)-stabilized $\alpha \mathrm{S}$ oligomers and $\alpha \mathrm{S}$ fibrils, suggesting that potential structural homology might be shared by these two types of $\alpha \mathrm{S}$ aggregates.

We also found that some residues like D119 and E139 at C-terminus showed the decreasing pattern. Ser129 also experienced a clear decrease of peak intensities along the exchange time. The behavior of these $\mathrm{C}$-terminal residues suggested that they might experience protection during the first exchange period, which was also indicated by the peak intensities of first HSQC spectrum of the oligomer sample compared with the monomer reference [Figure 3.36. A]. But, like Ser129, the same situation was also observed for Ser9 at N-terminus, which could be due to the effect of residue side chain. This can also indicate that under current conditions, the differentiation between residues from different parts of $\alpha \mathrm{S}$ oligomers were still not significant and far from the precise required for quantitative analysis. Especially, the side effects from individual side-chain and their neighbor residues still largely affected the exchange process and might be more pronounced than the structural effects that we expected to observe.

Comparing to the results of $\mathrm{H} / \mathrm{D}$ exchange for fibrils of E46K and $\mathrm{A} 53 \mathrm{~T} \alpha \mathrm{S}$ fibrils, the compounds-stabilized oligomers displayed much weaker protection against solvent exchange. Even under exchange condition i.e. $70 \% \mathrm{D}_{2} \mathrm{O}$ and 5 minutes forward exchange time, we still didn't observe the expected difference of protection at different regions of $\alpha \mathrm{S}$. In $\alpha \mathrm{S}$ fibrils, hydrogen bond is a major factor that protect amide proton against solvent exchange. Thus, the weaker protection of the oligomers suggests that the compounds-stabilized oligomers don't contain the regions that are enriched in hydrogen bonds.

Another factor we need to take into account was that after 3 days aggregation with compounds under the given condition, maximally $30 \% \alpha \mathrm{S}$ monomers would be converted into NMR-invisible oligomers measured by the 1D NMR monomerconsumption assay. Due to the heterogeneity and continuum distribution of oligomeric 
species, the effective population of oligomers that finally contributed to our observation during the exchange could be far less than $30 \%$ of total amount of $\alpha \mathrm{S}$. Thus the corresponding signals from oligomers were not enough to be distinguished from dominating monomer signals.

In summary, we optimized H/D exchange methods for probing conformation of the compounds-stabilized $\alpha \mathrm{S}$ oligomers. After the stepwise improvement for each experimental condition, we observed that the residues from NAC region displayed slight solvent protection. But the general protection of the compounds-stabilized oligomers was much weaker than that of fibrils, suggesting that the compoundsstabilized oligomers likely don't contain strongly hydrogen-bonded regions like fibrils. 


\section{Discussion}

\subsection{Functional relevance of alpha-Synuclein phosphorylation}

\subsubsection{The effect of PD-associated mutations on the phosphorylation of $\alpha$ - Synuclein}

Although $\alpha \mathrm{S}$ phosphorylation has been implicated in the pathogenesis of $\mathrm{PD}$, little is known about effects of PD-associated mutations (A30P, A53T and E46K) on the phosphorylation of $\alpha \mathrm{S}$, or vice-versa. The aim of this section was to address this gap in knowledge and to provide insights into the impact of the PD-associated mutations on phosphorylation of $\alpha \mathrm{S}$ at S87 and S129 in vitro. This was achieved by real-time NMR method and by employing kinases that are known to specifically and efficiently phosphorylate $\alpha \mathrm{S}$ at these residues, namely PLK2 (S129) and CK1 (S87 and S129).

Our quantitative analysis of phosphorylation kinetics revealed that $w t-\alpha \mathrm{S}$ was phosphorylated at S129 faster and slightly higher levels than all three genetic variants by using the same kinase and protein concentrations (Figures 3.1.1 and 3.1.2). Analogous findings regarding the in vitro phosphorylation of the pathogenic A30P and A53T $\alpha$ S by CK2 were reported previously (Ishii et al, 2007). Both A30P and A53T were reported to be phosphorylated in vitro at a slower rate compared to $w t-\alpha \mathrm{S}$. It is noteworthy that the extent of CK2-mediated phosphorylation of $\alpha \mathrm{S}$ does not exceed 5$10 \%$. However, we observed that none of the PD mutants seemed to influence the kinetics or extent of phosphorylation at S87 (Fig. 2B).

The above observations raise questions about why the disease-related mutants affect the levels of phosphorylation of S129 but not S87. The answer to this question may lie in differences in the structural consequences of the S87 and S129 phosphorylation. The previous studies indicated that $\alpha \mathrm{S}$ phosphorylation at S87, but not S129, interferes with $\alpha \mathrm{S}$ binding to membranes (Paleologou et al, 2010). It is also likely that the conformational changes resulting from the amino acid substitution in the 
disease mutants affect their interaction with kinases and/or phosphatases. It has previously been shown that the A30P mutation disrupts a region of residual helical structure that exists in the $w t$ protein, whereas the A53T mutation results in a slight enhancement of a small region around the site of mutation with a preference for extended conformations (Bussell \& Eliezer, 2001). Furthermore, the A30P and A53T mutations slightly weaken transient interactions between the acidic C-terminal tail of $\alpha \mathrm{S}$ and its $\mathrm{N}$-terminal region (Bertoncini et al, 2005b). In contrast, the E46K mutation enhances such contacts (Rospigliosi et al, 2009).

\subsubsection{Functional relevance of the Ser129 phosphorylation of $\alpha$-Synuclein}

Several posttranslational modifications of $\alpha \mathrm{S}$ have been described, including phosphorylation, nitration, ubiquitination, and truncations, but the role of these modifications in modulating the physiologic and/or pathological properties of $\alpha$-syn remains poorly understood. One can speculate that many $\alpha \mathrm{S}$ properties and functions are regulated by its interactions with other proteins and its subcellular localization/targeting, which in turn might be regulated by posttranslational modifications. Therefore, identifying the key molecules responsible for regulating $\alpha \mathrm{S}$ post-translational modifications and elucidating their mechanism of action are essential for unraveling the molecular basis of $\alpha \mathrm{S}$ function(s) in health and disease.

Of the posttranslational modifications, phosphorylation at the Ser129 is the major modification of $\alpha \mathrm{S}$, which nearly $90 \% \alpha \mathrm{S}$ in Lewy body was phosphorylated at the Ser129 (Anderson et al, 2006; Fujiwara et al, 2002; Okochi et al, 2000). Therefore, we investigated the selectivity and efficiency of PLKs-mediated phosphorylation of $\alpha \mathrm{S}$. Our kinetics data indicated that both PLK2 and PLK3 could phosphorylate $\alpha \mathrm{S}$ with high efficiency and selectivity at the Ser129, especially PLK3 could achieve 100\% phosphorylation of the Ser129 within 2.5 hours.

At the same time, the different phosphorylation kinetics of Ser129 by PLK1-3 suggest that "the propensity of PLKs to phosphorylate $\alpha$-syn is determined by their substrate specificity and sequence complementarities between the catalytic site of each kinase and the amino acid sequence flanking the phosphorylated serine residue as well 
as the conformation of the protein. Sequence analysis and three-dimensional homology modeling studies revealed that PLK2 and PLK3 show the highest pairwise identity of the catalytic domain and ATP binding site. The sequence identity between PLK2 and PLK3 at the catalytic domain and ATP binding site is 68 and 97\%, and that of PLK2 and PLK3 to PLK1 is \%53 and 86-90\%, respectively (Johnson et al, 2007). Furthermore, the consensus sequences of PLK2 and PLK3 are very similar and characterized by the presence of acidic residues within the 3-4 residues flanking the phosphorylation site $((\mathrm{D} / \mathrm{E}) \mathrm{X}(\mathrm{S} / \mathrm{T}) \theta(\mathrm{D} / \mathrm{E})$, where $\theta$ denotes a hydrophobic amino acid. PLK1 to 3 have a preference for substrates in which at least one of the three residues flanking the serine residue is negatively charged. Both $\alpha \mathrm{S}$ and $\beta \mathrm{S}$ fulfill this criterion. This charge distribution on the substrates complements the electropositive distribution on the substrate binding sites of PLK1 to 3" ((Mbefo et al, 2010), p.2818, co-authored).

As it's already proved that PLK2 and PLK3 were responsible to phosphorylate $\alpha \mathrm{S}$ with high specificity and efficiency at Ser129, the next question is whether phosphorylation of Ser129 could affect $\alpha \mathrm{S}$ aggregation. This is of crucial aspects to evaluate functional relevance of the Ser129 phosphorylation. Thus, we generated pS129- $\alpha$ S by PLK3 and the mimics of the Ser129 phosphorylation P128E/S129E and M127E/P128E/S129E by genetic mutations. Both the phosphorylated $\alpha \mathrm{S}$ and its two mimicking mutants showed very close aggregation kinetics and morphology to $w t \alpha \mathrm{S}$, strongly suggesting that the phosphorylation at Ser129 of $\alpha \mathrm{S}$ didn't affect the aggregation. Similar finding has also been reported that phosphorylation of Ser129 didn't change the aggregation propensity of $\alpha \mathrm{S}$ (Waxman \& Giasson, 2011). A structural reason that Ser129 phosphorylation of $\alpha \mathrm{S}$ would not affect its aggregation is that Ser129 is located at $\alpha$ S C-terminus. C-terminal (residue 100-140) is well known for its highly disordered state in both monomeric and fibrillar states. Thus, even embedded into the fibrils, C-terminal of $\alpha \mathrm{S}$ still retains its disordered conformation outside the fibril core and thereby spatially offers chance for kinase recognition. This feature is drastic to the Ser87, which is located in central part of NAC and deeply buried inside of the rigid fibril core. Because of this special location, Ser87 is very hard to be accessed by the kinase at its fibrillar state. Vice versa, if monomeric $\alpha \mathrm{S}$ was phosphorylated at Ser87, then the fibrillization could be blocked due to one strong polar group was 
introduced into the core region responsible for the fibril formation (Paleologou et al, 2010).

Moreover, several line of evidence have already pointed out that $\alpha \mathrm{S}$ fibrils could be phosphoryalted by PLK1-3 in vitro (Mbefo et al) and in vivo (Waxman \& Giasson, 2011). These findings indicate that $\alpha \mathrm{S}$ could also be phosphorylated after they aggregated into fibrils in Lewy body. Therefore, we speculate that the aggregation and the phosphorylation of Ser129 are two independent events in physiological scenario of $\alpha \mathrm{S}$. This indicates that pathological effects of the Ser129 phosphorylation of $\alpha \mathrm{S}$ might not rely on modifying pathway of its aggregation. The question that why $90 \% \alpha \mathrm{S}$ deposited in Lewy Body were phosphorylated at the Ser129 still needs to be further addressed in future research.

\subsection{Rab proteins bind to alpha-Synuclein and modulate its aggregation}

Lindquist group found: (1) Rab1, Rab3a and Rab8a could suppress the disruption of vesicle trafficking at multiple steps caused by $\alpha \mathrm{S}$; (2) The cellular Rab homeostasis was disturbed by $\alpha \mathrm{S}$ (Cooper et al, 2006; Gitler et al, 2008). To unearth the molecular mechanisms underlying these findings, we implemented the protein interaction studies for $\alpha \mathrm{S}$ and the different Rab proteins by NMR spectroscopy and the aggregation assays for $\alpha \mathrm{S}$ in the presence of Rab proteins.

\subsubsection{Rab8a binds to monomeric $\alpha \mathrm{S}$}

\subsubsection{Rab8a binds to C-terminus of $\alpha S$}

NMR titration identified that only Rab8a binds to monomeric $\alpha \mathrm{S}$ in solution, however Rab1b and Rab3a lack binding. At the same time, our investigations revealed that both GDP- and GppNHp-bound Rab8a could bind to C-terminal of $\alpha \mathrm{S}$, but interestingly Rab8a(GDP) binds to $\alpha \mathrm{S}$ with a stronger affinity than Rab8a(GppNHp). By sequence analysis for Rab8a, we found that Rab8a was relatively positive-charged and predicted that both C-terminal loop and N-terminally folded part of Rab8a were capable of 
binding to $\alpha \mathrm{S}$ independently. This has been proved by our NMR titration of $\mathrm{C}$ terminally truncated $\operatorname{Rab} 8 \mathrm{a}-\delta \mathrm{C}$ and the Rab8a C-terminal peptide into ${ }^{15} \mathrm{~N}-\alpha \mathrm{S}$. As addressed in the literature of Lindquist, they found that only Rab3a and Rab8a similar to Rab1 could protect against cellular toxicity induced by $\alpha \mathrm{S}$ in primary rat midbrain cultures and C. elegans. (Gitler et al, 2008). In C. elegans., Rab8a uniquely displayed the strongest rescuing effect characterized by extending lifespan of the worms. Correlating our NMR titration data, it therefore suggests that the molecular interaction between $\alpha \mathrm{S}$ and Rab8a might play an important role in the rescuing mechanism of Rab8a against $\alpha \mathrm{S}$ toxicity in the animal models.

Even the weaker amelioration to the $\alpha \mathrm{S}$ toxicity displayed by Rab1 and Rab3a in C. elegans., it's noteworthy that these two Rab proteins can also suppress the $\alpha \mathrm{S}$ toxicity in rat midbrain culture as similar extent as Rab8a. This suggests that these 3 Rabs are capable of protecting the $\alpha \mathrm{S}$-induced toxicities with close extent but probably with different mechanisms. One speculation could be that $\alpha \mathrm{S}$ indirectly interacts with effector or regulator proteins of Rab1 and Rab3a rather than the direct binding. Recent study demonstrated that vesicle trafficking was modulated by the interaction between $\alpha \mathrm{S}$ and prenylated $\mathrm{Rab}$ acceptor protein 1(PRA1) (Lee et al, 2011). This protein interaction was proposed to inhibit the recycling between GDP and GTP bound Rab and thereby interfered the related vesicle trafficking. PRA1 is a protein comprising both transmembrane and cytosol domains and located at ER and Golgi. It interacts with prenylated Rab-GTPases and facilitates Rabs anchored at the membrane at active GTPbound state to play its function on regulating vesicle trafficking (Figueroa et al, 2001; Hutt et al, 2000). Particularly, it's shown that PRA1 binds to Rab1, Rab3 and Rab5 (Bucci et al, 1999; Martincic et al, 1997).

Another speculation is that Rab1 and Rab3a modified the $\alpha \mathrm{S}$ toxicities by modulating $\alpha \mathrm{S}$ aggregation rather than direct interactions with $\alpha \mathrm{S}$ monomers. This issue will be discussed in section 4.2.2.

\subsubsection{2 $\alpha$ S binds to the Switch I region of Rab8a}

The interaction mechanism of Rab8a and $\alpha \mathrm{S}$ was further coherently shown by titrating $\alpha \mathrm{S}$ into ${ }^{15} \mathrm{~N}$-Rab8a and we thereby mapped the binding sites on Rab8a. There were two 
regions of Rab8a, Switch I region and C-terminus, mainly involved in the binding to $\alpha \mathrm{S}$. For Switch I region, we observed increased chemical shift perturbation and enhanced intensity as the response to the addition of $\alpha \mathrm{S}$ [Figure 3.2.20], indicating that $\alpha \mathrm{S}$ binds and restricts the chemical exchange of this region. Switch I is an important region responsible for interactions of Rab8a with guanine nucleotide exchange factors (GEFs) and effector proteins (Itzen \& Goody, 2011). As a common feature, the GEFs and effectors interacts with specific sites of Switch I and Switch II region and hence induce the significant structural changes to these two regions and the interswitch region. Therefore, the switch regions were enriched in potent binding sites for different binding partners and they are structurally sensitive to external bindings. $K_{d}$ measured for Rab8a(GDP) binding to $\alpha \mathrm{S}$ quantitatively depicted the binding strength for this proteinprotein interaction. As the $\mathrm{K}_{\mathrm{d}}$ for Rab8a binding to $\alpha \mathrm{S}$ is at the order of $\sim 100 \mu \mathrm{M}$, this is normally in the range of weak binding and much weaker than the common binding partners like GEFs and effectors in the Kd range of nM. Therefore, in real systems like neurons, $\alpha \mathrm{S}$ likely needs to compete with these stronger binding partners of Rab8a. However, $\alpha \mathrm{S}$ is much more abundant than GTPases in cells. The large excess of $\alpha \mathrm{S}$ could favor the binding to Rab8a. Especially, under the pathological conditions, $\alpha \mathrm{S}$ would progressively accumulate at the vesicles.

According to the definition of the Switch regions, Switch I and Switch II regions experience significant structural changes between GDP and GTP states as a common feature conserved in Ras family and thus these two regions were named with "Switch" (Lee et al, 2009; Milburn et al, 1990; Schlichting et al, 1990). Our titration data show that Switch I region of Rab-GDP was significantly affected by the binding of $\alpha \mathrm{S}$ and the following interswitch region also display slight increase in peak intensities [Figure 3.2.20]. This observation suggests that a major binding site was located in Switch I region and the interswitch region was also allosterically modified by the structural change in Switch I. Notably, the conformational nucleotide-dependence of the switch regions describes that for GTP state, Switch regions usually adopts more structured conformation and expose the hydrophobic patch to favor the binding of effectors. Contrarily, Switch regions present as more disordered conformation at GDP state (Itzen \& Goody, 2011). Thus, this conformational nucleotide-dependence can provide the 
structural basis to understand the preference for $\alpha \mathrm{S}$ binding to Rab8a as we identified that Switch I region of Rab8a serves as major binding site for $\alpha \mathrm{S}$.

As described in section 3.2.2.1, the $\mathrm{K}_{\mathrm{d}}$ for $\mathrm{Rab8a}(\mathrm{GppNHp})$ binding to $\alpha \mathrm{S}$ is approximately 2.5 fold higher than the binding of Rab8a(GDP) to $\alpha \mathrm{S}$, suggesting that Rab8a(GDP) possesses a stronger binding affinity to $\alpha \mathrm{S}$ than Rab8a(GppNHp). And, this difference was also unambiguously shown in the NMR titration of C-terminally truncated Rab8a(6-176) into ${ }^{15} \mathrm{~N}-\alpha \mathrm{S}$ as shown in section 3.2.2.3 [Figure 3.2.6]. For all the molar ratios that we used for titrating Rab8a(6-176) into ${ }^{15} \mathrm{~N}-\alpha \mathrm{S}$, Rab8a- $\delta \mathrm{C}(\mathrm{GDP})$ induced larger chemical shift perturbation to $\alpha \mathrm{S}$ than Rab8a- $\delta \mathrm{C}(-\mathrm{GppNHp})$. Taken together, these observations indicate that there is a structural preference for $\alpha \mathrm{S}$ binding to folded part of Rab8a. Importantly, as we identified that Switch I region of Rab8a was a major site for $\alpha \mathrm{S}$ binding, this structural preference can attribute to the significant nucleotide-dependence of conformation of the Switch regions and the interswitch region.

Moreover, more disordered conformations are commonly present to Switch regions at GDP state might be more favorable for binding of $\alpha \mathrm{S} \mathrm{C}$-terminal, whereas more structured conformation of Switch regions at GTP state is usually favorable for effector protein binding (Itzen \& Goody, 2011). There are two functional relevance of this structural preference of Rab8a binding to $\alpha \mathrm{S}$ can be taken into account. $\alpha \mathrm{S}$ preferentially binds to Rab8a(GDP), which leads the active Rab8a, namely Rab8a(GppNHp), to be less affected by the presence of $\alpha \mathrm{S}$. This serves as a protection mechanism to effects of $\alpha \mathrm{S}$ on normal function of Rab8a, which supports the existing results that over-expression of Rab8a can suppress the cellular toxicity of $\alpha \mathrm{S}$. However, there should be a well-balanced equilibrium existing between the GDP- and GTP-bound Rab8a. If large excess of $\alpha \mathrm{S}$ is present in the cytosol it could bind to Rab8a-GDP and disturb the equilibrium of Rab8a between the GDP and GTP states and. Thus the reduced pool of Rab8a(GTP) can cause the trafficking deficits which could be viewed as one phenotype of $\alpha \mathrm{S}$-induced cellular toxicity.

\subsubsection{3 $\alpha$ S binds to the C-terminus of Rab8a}

Another major binding site is C-terminus of Rab8a (Asp176-Arg205). We considered this region due to its positive net charge, which is capable of compensating the negative 
charges enriched in the C-terminal region of $\alpha \mathrm{S}$. We further identify this charge-driven interaction mechanism by our NMR titration assays. Titration of the synthesized Cterminus peptide of Rab8a into ${ }^{15} \mathrm{~N}-\alpha \mathrm{S}$ could reproduce the effects of full-length Rab8a binding to $\alpha \mathrm{S}$ [Figure3.2.4]. Correspondingly, this interaction has also been observed at C-terminus of Rab8a when we titrate $\alpha \mathrm{S}$ into the full-length ${ }^{15} \mathrm{~N}$-Rab8a [Figure3.2.20]. Especially, the binding of $\alpha \mathrm{S}$ restricted the chemical exchange of Rab8a C-terminal reflected by the intensity plot at 8:1 molar ratio of $\alpha \mathrm{S}$ to Rab8a(GDP), which was similar to the response of Switch I region of Rab8a-GDP. The C-terminus of Rab8a is structurally disordered and functionally responsible for the membrane-anchoring through prenylation of the cysteine in the CVLL motif. The ${ }^{1} \mathrm{H}-{ }^{15} \mathrm{~N}$ HSQC spectra of the peptide and full-length Rab8a were not fully overlapped, suggesting that this terminal region possesses contacts with other parts of protein. But, in comparison to the binding of $\alpha \mathrm{S}$ to Switch I region in the N-terminally folded part, the C-terminus of Rab8a is highly disordered loop and less affected by the different nucleotide binding. Hence, even at GTP state this C-terminal region can still largely contribute to the interaction between Rab8a and $\alpha \mathrm{S}$, although the conformation of Switch I region is unfavorable for the binding of $\alpha \mathrm{S}$. Taken together, the C-terminus of Rab8a play an important role in the interaction of Rab8a and $\alpha \mathrm{S}$ due to its positive net-charge and relatively conformational independence of the nucleotide-binding. The characters of this $\mathrm{C}$ terminus enable Rab8a to bind to $\alpha \mathrm{S}$ at both GDP and GTP states. Furthermore, due to the binding of $\alpha \mathrm{S}$, the structural contacts between disordered $\mathrm{C}$-terminal and the structured part of Rab8a might be affected and the thereby the external perturbation could be partially introduced to the tertiary structure of Rab8a.

Additionally, we found that both Rab8a- $\delta \mathrm{C}$ and the C-terminal peptide of Rab8a can bind to $\alpha \mathrm{S}$. But, we also observed that the binding strengths of these two fractions to $\alpha \mathrm{S}$ were weaker than the full-length protein according to the chemical shift perturbation. Therefore, these observations suggest that the Switch I region and Cterminus of Rab8a are simultaneously contributing to the binding of full-length Rab8a to $\alpha \mathrm{S}$. 


\subsubsection{The Ser129 phosphorylation enhances the binding of $\alpha \mathrm{S}$ to Rab8a}

Phosphorylation of $\alpha \mathrm{S}$ at Ser129 is one of most common posttranslational modifications to $\alpha \mathrm{S}$. As we discussed in section 3.1 and 4.1, the functional role of this phosphorylation is still less defined. Within the scope of this dissertation, two major questions for the Ser129 phosphorylation of $\alpha \mathrm{S}$ remain to us: 1) what are the physiological functions of this phosphorylation; 2) what is the pathological relevance of this phosphorylation in synucleinopathies.

Herein, base on the NMR titration, we found that the Ser129 phosphorylation enhanced the binding of $\alpha \mathrm{S}$ to Rab8a in comparison to $w t$ - $\alpha \mathrm{S}$ [Figure 3.2.4]. The phosphorylation modified the side chain of Ser129 by introducing one negative charge. The length of side chain of Ser129 was increased and the phosphate group attached on the end of Ser129 side chain possesses stronger electronegativity than side chains of normal amino acids. These factors can be the reasons for the binding enhancement.

To date, most of studies on the Ser129 phosphorylation of $\alpha \mathrm{S}$ were mainly addressing the questions based on the level of cell culture and other animal models. These assays can study the Ser129 phosphorylation in more physiological environments. But, they are less efficient to identify and determine the specific mechanism at molecular level (Braithwaite et al). Recently, V. Sancenon et al. reported that phosphorylation of $\alpha \mathrm{S}$ at Ser129 could reduce vesicle trafficking defects caused by $\alpha \mathrm{S}$ (Sancenon et al, 2012). In this paper, the authors also reported that the level of Rab5 was increased as a marker of the endosome abnormality occurred in this pathological process. This implicate that there might be a pathological connection between RabGTPases and $\alpha \mathrm{S}$ in the scenario of vesicle trafficking deficits induced by $\alpha \mathrm{S}$. However, the kinases used in this paper were CK family kinases. CKs normally phosphorylate $\alpha \mathrm{S}$ not only at Ser129 but also Ser87 as we described in section 3.1.1. Thus, it should not be excluded that the effects observed in the paper might also be result from the Ser87 phosphorylation. Moreover, the impacts of the Ser129 phosphorylation on the vesicle trafficking deficits were defined based on genetic background and the specific interactions involved in the observations were not recognized in the paper. Compensating to these two points, our in vitro investigation was based on $\alpha \mathrm{S}$ with the phosphorylation specifically at Ser129 by PLK3. At the same time, our investigation 
identified the enhancement effects of Ser129 phosphorylation on $\alpha$ S-Rab8a interaction unambiguously by NMR spectroscopy at atomic resolution. Hence, this enhancement mechanism observed by us suggests that the Ser129 phsophorylation of $\alpha \mathrm{S}$ likely plays an important role in the interaction with Rab8a and thereby further mediates $\alpha \mathrm{S}$ toxicity in the context of vesicle trafficking disrupted by the large accumulation of $\alpha \mathrm{S}$. Additionally, this is also the first specific indication based on protein-protein interaction to demonstrate that how phosphorylation at Ser129 of $\alpha \mathrm{S}$ mechanistically mediates the functions of $\alpha \mathrm{S}$.

\subsubsection{Overview for the molecular interaction of $\alpha S$ and Rab8a}

In section 4.2.1.1 - 4.2.1.4, we in-depth discussed the mechanism of the interaction between $\alpha \mathrm{S}$ and Rab8a. Rab8a binds to $\alpha \mathrm{S} \mathrm{C}$-terminal through its $\mathrm{C}$-terminus and Switch I region and the electrostatic interaction is a major driven force for the binding. This binding has been well characterized from both $\alpha \mathrm{S}$ and Rab8a sides. At the same time, this binding displays nucleotide-dependence mechanism that Rab8a-GDP binds to $\alpha \mathrm{S}$ stronger than Rab8a-GppNHp. As the Switch I region of Rab8a was identified as a major binding site, this mechanism can attribute to the structural preference of the Switch I region for $\alpha \mathrm{S}$ binding. Furthermore, we identified that the phosphorylation of $\alpha \mathrm{S}$ Ser129 could enhance the binding between $\alpha \mathrm{S}$ and Rab8a, which is as a specific indication of the functional relevance of the Serr129 phosphorylation of $\alpha \mathrm{S}$.

In concert with our in vitro investigation, the in vivo assays have been undertaken by our collaborator Prof. Dr. Tiago F. Outeiro in his lab. Both $\alpha$ S and Rab8a were co-expressed in the model system of H4 neuroglioma cells (McLean et al, 2000) and co-localization of these two proteins has been observed. Hence, the co-localization suggests that these two proteins have potential interactions in the cells, which is in agreement with our in vitro finding. Furthermore, to identify the deeper mechanism that the Ser129 phosphorylation mediates the interplay between $\alpha \mathrm{S}$ and Rab8a, one phosphorylation mimic S129D $\alpha \mathrm{S}$ has been proposed by us to test its co-localization with Rab8a in vivo and interaction with Rab8a in vitro.

Up to now, the specific protein-protein interactions characterized from both interaction partners at atomic resolution for $\alpha \mathrm{S}$ are still rare. Thus, our studies are not 
only helpful in understanding the rescuing mechanism of Rab8a against the $\alpha \mathrm{S}$ toxicities, but also extend our knowledge respecting to general principles for the $\alpha \mathrm{S}$ participating protein-protein/ligand interactions.

\subsubsection{Rab proteins modulate the $\alpha \mathrm{S}$ aggregation}

Investigating $\alpha$-Synuclein aggregation is one important approach to evaluate the physiologic and pathologic relevance of $\alpha \mathrm{S}$ under the given conditions. We found that Rablb and Rab3a didn't bind to $\alpha \mathrm{S}$ monomers but modulated $\alpha \mathrm{S}$ aggregation. Rab8a can bind to $\alpha \mathrm{S}$ as well as modulate $\alpha \mathrm{S}$ aggregation. The effect of Rab8a on $\alpha \mathrm{S}$ aggregation could be reproduced by its C-terminal peptide. Moreover, these 3 Rabs adjusted $\alpha \mathrm{S}$ aggregation mainly in a dose-independent manner. To analyze the molecular mechanism of the 3 Rab proteins underlying their aggregation modulation effects, we take into account two types of molecular interactions: 1) the interaction of Rabs with monomeric $\alpha S$; 2) the interaction of Rab with aggregation intermediates of $\alpha \mathrm{S}$.

\subsubsection{Rab8a modulates $\alpha S$ aggregation}

Firstly, our titrations indicate that both GDP-bound and GTP-bound Rab8a can bind to $\alpha \mathrm{S} C$-terminal and introduce the structural perturbation to the local conformation of $\alpha \mathrm{S}$ C-terminal. At the same time, Rab8a can offer multiple binding sites, Switch I and Cterminus for $\alpha \mathrm{S}$, suggesting that Rab8a likely interact with $\alpha \mathrm{S}$ by $1: 2$ ratio of Rab8a/ $\alpha \mathrm{S}$. Thus, local concentration of $\alpha \mathrm{S}$ around Rab8a might be increased due to this binding. The increase of $\alpha \mathrm{S}$ local concentration could ficiliate nucleation and thereby shortening the lag phase.

Furthermore, the binding of Rab8a to $\alpha \mathrm{S}$ C-terminus might modify the longrange interactions between $\mathrm{N}$ and $\mathrm{C}$ termini of $\alpha \mathrm{S}$. As the long-rang interactions are important to maintain the protein conformation and inhibit aggregation of $\alpha \mathrm{S}$ (Bertoncini et al, 2005b; Dedmon et al, 2005c), the presence of Rab8a might affect $\alpha \mathrm{S}$ aggregation by modifying the long-range interactions due to its binding. In particular, this aggregation modulation mechanism of Rab8a by interacting with $\alpha \mathrm{S}$ C-terminal through the electrostatic interactions was confirmed by the aggregation assay of $\alpha \mathrm{S}$ with 
the addition of the positively charged Rab8a C-terminal peptide [Figure 3.2.10]. This modulation mechanism is similar to the case that $\alpha \mathrm{S}$ aggregation is mediated by positively charged polyamines (Fernandez et al, 2004). Furthermore, consistent with the observations by W. Hoyer et al. (Hoyer et al, 2004), our results indicate that C-terminal is important in modulating $\alpha \mathrm{S}$ aggregation although it's disordered and absent in fibril core.

The distinction between polyamines and Rab8a should be noticed. Polyamines are small molecules but Rab8a is a protein relatively larger than $\alpha \mathrm{S}$. This suggests that even Rab8a impacts on $\alpha \mathrm{S}$ aggregation in a polyamines-like manner, it also possessed some other characters specially modulating the aggregation in the way different to polyamines. Correlating with the binding of Rab8a to $\alpha \mathrm{S}$, C-terminus of Rab8a might act in a polyamine-like manner to modulate $\alpha \mathrm{S}$ aggregation. In addition, the globular part can also bind to $\alpha \mathrm{S} \mathrm{C}$-terminus and potentially affect the aggregation as well. Thus the full-length Rab8a that is a combination of its globular part and C-terminal loop might interact with $\alpha \mathrm{S}$ and adjust its aggregation by a more collective way.

\subsubsection{Rablb and Rab3a modulate $\alpha$ S aggregation}

From the interaction studies by NMR, we found that no molecular interactions between monomeric $\alpha \mathrm{S}$ and Rab1b/Rab3a. But, they did enhance the $\alpha \mathrm{S}$ aggregation by shortening the lag phase and significantly prompting the elongation. In addition, when the fibrillar $\alpha \mathrm{S}$ was titrated into ${ }^{15} \mathrm{~N}-\mathrm{Rab} 1 \mathrm{~b}-\delta \mathrm{C}$ we did not observe the pronounced perturbation to Rablb either. Therefore, it's likely that Rablb binds to $\alpha \mathrm{S}$ aggregation intermediates. This interaction might rely on the fact that surface or shape of Rablb fit to conformation of $\alpha \mathrm{S}$ oligomers, which enables Rablb to interact with the $\alpha \mathrm{S}$ oligomers. Similarly, HSP70 were reported to selectively interact with $\alpha \mathrm{S}$ aggregation intermediates and affect the $\alpha \mathrm{S}$ aggregation (Dedmon et al, 2005a; Huang et al, 2006; Roodveldt et al, 2009).

At the same time, we observed that all 3 Rabs could enhance the elongation rate of $\alpha \mathrm{S}$ significantly. This might originate from the specific protein conformation which is conserved amongst the members of Rab-GTPases family. The aggregation control of $\alpha \mathrm{S}$ with BSA also suggests that the aggregation mediation effects of Rablb should be a 
result specifically from Rab1b. Furthermore, 3 Rabs affected the $\alpha \mathrm{S}$ aggregation mainly by a dose-independent manner within the concentration ranges that we applied. The addition of 0.3 to 3 molar equivalents of Rabs did not cause significantly difference in the aggregation behavior of $\alpha \mathrm{S}$. As Rab1b and Rab3a were suggested to selectively interact with $\alpha \mathrm{S}$ aggregation intermediates, the concentration of Rabs even as half of the $\alpha \mathrm{S}$ monomer concentration is still likely beyond the concentration of the $\alpha \mathrm{S}$ intermediates because the oligomeric species of $\alpha \mathrm{S}$ is populated much less than monomers in solution (Conway et al, 2000). Therefore, similar to the case of HSP70 (Dedmon et al, 2005a), there is putatively a critical concentration for Rabs with respect to $\alpha \mathrm{S}$ concentration to play its role in modulating $\alpha \mathrm{S}$ aggregation, particularly for Rab1b and Rab3a. If the concentration of Rabs is higher than this critical value, it is speculated that the amount of Rabs is enough to catalyze the fibril growth of $\alpha \mathrm{S}$ through binding to the $\alpha \mathrm{S}$ oligomers formed during the lag phase.

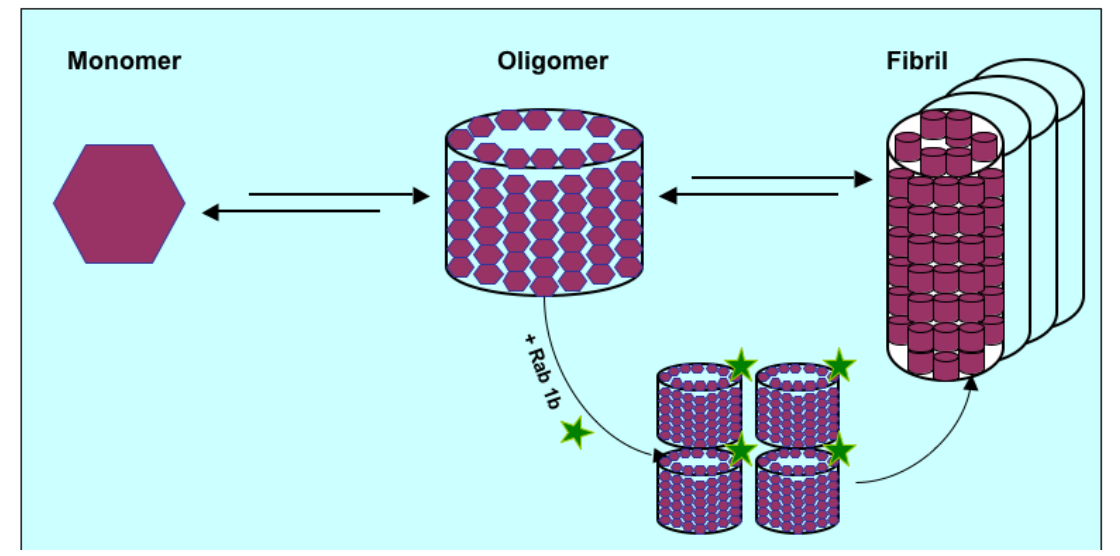

Figure 4.2.1 Putative role of Rab1b in modulating $\alpha \mathrm{S}$ aggregation. The aggregation pathway consisting of the oligomerization and fibrillization has bee depicted, in where Rablb selectively binds to the oligomeric species and favors the assembly of the oligomers into fibrils.

Taken together, we speculate that Rab1b and Rab3a modulate $\alpha \mathrm{S}$ aggregation by interacting with $\alpha \mathrm{S}$ oligomers and facilitate the assembly of oligomers to larger oligomeric species or directly into the fibrils [Figure 4.2.1]. This can explain why the Rabs could modify aggregation by both shortening lag phase and prompting fibril growth. Rab8a due to its binding to monomeric $\alpha \mathrm{S}$ might perform a different mechanism in modulating $\alpha \mathrm{S}$ aggregation in comparison to Rab1b and Rab3a. 


\subsubsection{Pathological implications of Rabs modulating $\alpha S$ aggregation}

Vesicle trafficking deficits caused by $\alpha \mathrm{S}$ have been implicated in the Synucleinopathies. Elevated expression of $\alpha \mathrm{S}$ was reported to affect the vesicle trafficking from ER to Golgi as the vesicle docking and fusion to Golgi membrane were blocked and the accumulation of undocked vesicles grew into massive intracellular clusters (Cooper et al, 2006; Gitler et al, 2008). Rab1 is responsible for modulating the vesicle trafficking between ER and Golgi. The overproduction of Rab1 gene in vivo was observed to suppress the trafficking disruption casued by $\alpha \mathrm{S}$. (Cooper et al, 2006) In addition, Rab8a, which is located at the post-Golgi vesicles and Rab3a, which is expressed specifically in neurons, could also suppress this cellular toxicity of $\alpha \mathrm{S}$ by their overexpression, suggesting that $\alpha \mathrm{S}$ broadly affects multiple steps of vesicle trafficking in cells (Gitler et al, 2008). Furthermore, recent study points out that $\alpha \mathrm{S}$ aggregation induced the aggregation of many Rab-GTPases in the model system of $S$. cerevisiae (Soper et al, 2011), as the direct evidence of the interactions between $\alpha \mathrm{S}$ and $\mathrm{Rab}$ proteins in vivo. Thus, the tight interplays between $\alpha \mathrm{S}$ and the Rab proteins have been implicated in the scenario of vesicular trafficking.

Both the Rab1 mutation (Wilson et al, 1994) and the overexpression of $\alpha \mathrm{S}$ (Gosavi et al, 2002) could cause the Golgi fragmentation (Cooper et al, 2006). This reflects a deleterious relationship that $\alpha \mathrm{S}$ can reproduce the harmful effects of the Rab1 malfunction caused by the critical mutation. Conversely, this also partially explains that Rab1 was capable of rescuing the toxic effects of $\alpha \mathrm{S}$ involved in the ER-Golgi transport. The mechanism of $\alpha \mathrm{S}$-induced deficits of vesicle trafficking and cellular compartment fragmentation might be attributed to the high affinity of $\alpha \mathrm{S}$ to acidic phospholipid-rich membranes and the prone of misfolding and oligomerization (Cooper et al, 2006). In vitro biophysical studies found that the binding of $\alpha \mathrm{S}$ monomers to the artificial vesicle, SUV, can render the deformation of SUV membrane (Bodner et al, 2009), implicating that the attenuated membrane intactness likely account for the vesicle dysfunction induced by $\alpha \mathrm{S}$. Meanwhile, $\alpha \mathrm{S}$ oligomers have been reported to possess pore-forming propensity in the membranes (Kim et al, 2009). The mitochondria fragmentation has been observed as a result of the $\alpha \mathrm{S}$ oligomerization as well (Exner et al, 2012; Kamp et al, 2010), which the fragmentation is probably similar in the toxic mechanism leading to 
the Golgi fragmentation by $\alpha \mathrm{S}$ and speculated as one general phenotype of $\alpha \mathrm{S}$ oligomers-induced toxicities. Thus, the vesicle trafficking disruptions caused by $\alpha \mathrm{S}$ (Cooper et al, 2006; Gitler et al, 2008) might be as the results of $\alpha \mathrm{S}$ binding to the vesicle membranes and the pore-forming effects of $\alpha \mathrm{S}$ oligomers.

Comparing to the soluble monomers and oligomers of $\alpha \mathrm{S}, \alpha \mathrm{S}$ fibrils possesses lower affinity to membrane and less cellular toxicity than the oligomeric species. Taken together, therefore, one molecular mechanism for the $\alpha$ S-toxicity suppression by the Rabs is proposed: Rabs lowered the populations of $\alpha \mathrm{S}$ oligomers by enhancing their conversation into fibrils and thereby the toxicity of $\alpha \mathrm{S}$ was reduced.

\subsection{Conserved fibril core and its implications to the conformation of alpha-Synuclein oligomers}

\subsubsection{Molecular structure and supermolecular assembly of $\alpha \mathrm{S}$ fibrils}

One central question of our study is to find the impact of 3 mutations A30P, E46K and A53T on the $\alpha \mathrm{S}$ fibril structure. To address this question, we need to determine if these 3 mutation sites are located in a structured $\beta$-sheet or in loop. At the same time, it also needs to answer if the arrangement of $\beta$-sheets inside fibril core would be changed by these 3 mutations.

\section{The locations of the $46^{\text {th }}$ and $53^{\text {rd }}$ residue inside $\alpha \mathrm{S}$ fibrils}

Herein, we determine a conserved fibril core which exists between E46K and A53T $\alpha$ S fibrils by using solution NMR based H/D exchange. Particularly, for both mutants, the $46^{\text {th }}$ residue was likely located in a loop region that between the first and second $\beta$-sheet. This is close to the observation for $w t$ and $\mathrm{A} 30 \mathrm{P} \alpha \mathrm{S}$ fibrils by combination of H/D exchange and HR-MARS (Cho et al, 2011). Riek group used solution NMR based H/D exchange together with ssNMR for rigid core region and cryo-EM for assembly of filaments depicted the inner arrangement of $\beta$-sheets inside core and the possible fold of $w t \alpha \mathrm{S}$ fibrils of both twisted and straight type of fibrils (Vilar et al, 2008). In this comprehensive study, the location of E46 was also identified in a loop region. Similar speculation that E46K was located in a loop region was 
proposed based on the ssNMR experiments for the fibrils of $w t \alpha \mathrm{S}$ as well (Heise et al, 2005). However, the indication from this ssNMR analysis is less unambiguous due to the incomplete assignments especially for the E46 and its neighbor region.

With improved resolution, the residues located in ambient region of E46 in $w t$ $\alpha \mathrm{S}$ fibrils and K46 in E46K $\alpha \mathrm{S}$ fibrils were assigned by ssNMR (Comellas et al, 2011). Based on the analysis of carbon chemical shifts of the ambient region for both $w t$ and $\mathrm{E} 46 \mathrm{~K} \alpha \mathrm{S}$, the mutation site of E46K was located in a structured region, the first $\beta$-sheet of fibril core. Meanwhile, the sequence for residue 40-51 completely conserved between human $\alpha \mathrm{S}$ and mouse $\alpha \mathrm{S}$. With improved resolution achieved by uniformly $\left[{ }^{13} \mathrm{C},{ }^{15} \mathrm{~N}\right]$, $\left[1-{ }^{13} \mathrm{C}\right]$ glucose and $\left[2-{ }^{13} \mathrm{C}\right]$ glucose labeled mouse $\alpha \mathrm{S}$ fibrils, this segment was fully assigned and the result also demonstrated that residue E46 was located in first $\beta$-sheet inside fibril core ( $\mathrm{Lv}$ et al). Thus, the agreement has still been not reached for the location of $46^{\text {th }}$ residue inside $\alpha \mathrm{S}$ fibrils. The related reasons that need to be considered for this discrepancy can be listed as: 1) the fibril samples for NMR measurements were generated under different solution conditions and thus might be differed partially between each other (Hoyer et al, 2002); 2) significantly structural heterogeneity of $\alpha \mathrm{S}$ fibrils has been witnessed by biphasic H/D exchange curves by solution NMR (Vilar et al, 2008) and doubling peaks for certain residues by ssNMR (Heise et al, 2005; Vilar et al, 2008), which is highly correlated different fibril morphologies. Especially, the region 44-75 where E46 is located was indicated with more flexibility and less structural homogeneity in recent ssNMR study on $\alpha \mathrm{S}$ fibril structure (Gath et al, 2012); 3) the methodological differences. For solution NMR based H/D exchange, the essence is to distinguish the solvent-exposured/protected regions of fibrils. But some structured parts in the fold of $\alpha \mathrm{S}$ fibrils might enjoy a interface with solvent and some loop regions might locate between the interface of two protofilaments and thereby less accessible by solvents (Comellas et al, 2011). ssNMR can achieve the in-situ analysis of fibril structure which is superior to H/D exchange method based on solution NMR, but the resolution and sensitivity of ssNMR can be affected by internal dynamics and heterogeneity of fibrils (Kloepper et al, 2007).

Different with the case of E46K, a better agreement for the location of mutation $\mathrm{A} 53 \mathrm{~T}$ in the fibril core has been reached. We identify that, for fibrils of both E46K and 
A53T $\alpha \mathrm{S}$ mutants, the locations of $53^{\text {rd }}$ residue are in second $\beta$-sheets in fibril core. This observation is close to other structural studies mentioned above (Cho et al, 2011; Comellas et al, 2011; Heise et al, 2005; Lv et al; Vilar et al, 2008).

\section{The impacts of the familiar mutations on $\alpha \mathrm{S}$ fibril core structures}

The structural comparison for E46K and A53T $\alpha \mathrm{S}$ fibrils are based on our H/D exchange results which point out that a conserved fibril core for them with respect to the basic arrangement of $\beta$-sheets and the boundaries of core. A very similar observation was obtained for $w t$ and A30P methods by similar method (Cho et al, 2011). This indicates that fibril core region is conserved between $w t \alpha \mathrm{S}$ and 3 familiar mutants. For the residues located at the starting and ending boundaries of fibril core, most of structural studies figure out fairly close result as we observed. And the boundaries which were started at $\sim 37^{\text {th }}$ residue and ended at $\sim 97$ residue were also further confirmed by the other techniques like EPR (Chen et al, 2007), Mass spectrum (Del Mar et al, 2005).

The central question of our study is to address that how the familiar mutations affect fibril structure. Particularly, E46K and A53T are in the core region of fibrils, unlike $\mathrm{A} 30 \mathrm{P}$ is located in $\mathrm{N}$-terminal loop. Our data suggest that there are minor changes caused by these two mutations on fibril structures. According to the chemical shift analysis, E46K and A53T mutations were reported to induce major and minor perturbation to fibril structure and attributed to their different side chains (Comellas et al, 2011). But, there was no further evidence from this work to point out mutationinduced change of $\beta$-sheets arrangement, suggesting that these two mutations probably impacted more on the local conformation around the single mutation points. One early result suggested that mutation A53T significantly affected on the fibril structure in comparison to $w t-\alpha \mathrm{S}$ (Heise et al, 2008). However, the critical regions for plausible comparison like residue50-60, 76-80 were not assigned for A53T fibrils.

\section{The role of different segments in the fibril formation}

Inspecting the $H / D$ exchange results, we found that the $4^{\text {th }} \beta$-sheet(70-81) experienced relatively stronger protection against solvent, indicating that this region is located in the center of the fibril fold. Meanwhile, the short $\beta$-sheet (63-65) and the fifth 
$\beta$-sheet (86-96) as the neighboring region of $4^{\text {th }} \beta$-sheet also displayed stronger protections [Figure 3.3.3]. These regions occupy most of NAC region of $\alpha \mathrm{S}$ known as pivot area responsible for aggregation. Particularly the $\beta 3$ and $\beta 4$ defined by our H/D exchange are highly overlapped with the $\beta 3$ and $\beta 4$ defined by Riek group (Vilar et al, 2008), where they indicated these two $\beta$-sheets are pivotal for fibril formations. The similar conclusions by other methods also point to that the certain segments in this region are centrally responsible for fibrillization(el-Agnaf \& Irvine, 2002; El-Agnaf et al, 1998; Giasson et al, 2001; Madine et al, 2008).

Interestingly, in our studies although $\mathrm{N}$-terminal residues (1-35) adopts a random coil conformation out of rigid fibril core, part of these residues still showed more protection compared to C-terminal, particularly the region 18 25. A recently ssNMR study on $\alpha \mathrm{S}$ fibrils suggested that the fibril core started from the first residue of $\mathrm{N}$ terminus (Gath et al, 2012). Although this finding is drastic to other structural investigation for $\alpha \mathrm{S}$ fibrils performed before, it still implicates the presence of potentially tight contact between N-terminal residues and fibril core. In agreement to this, Rienstra group recently evaluated the impact of A30P mutation on $\alpha \mathrm{S}$ fibril structure by ssNMR and identified that the fibril structure was mostly conserved between $\mathrm{A} 30 \mathrm{P}$ and $\alpha \mathrm{S}$ fibril. Nevertheless, this study found that due to mutation from Ala to Pro at $30^{\text {th }}$ residue, the non-ignorable chemical shift perturbation with a average value of 0.5ppm was observed for Val55-Val63 (Lemkau et al, 2012), suggesting that A30P interacts with this region either intra or intermolecularly or by some indirect means. Moreover, correlated with this observation, N-terminally truncated $\alpha \mathrm{S}$ was found to have a longer lag phase compared with $w t \alpha \mathrm{S}$ (Zibaee et al, 2007).

Except molecular structure of single $\alpha \mathrm{S}$ present in the fibrils, another intriguing question is how amino acid sequence affects the morphology of amyloid fibrils. As shown in Figure 3.3.1, the morphologies of E46K and A53T $\alpha \mathrm{S}$ fibrils reflected by EM were fairly different. However, according to our H/D exchange data, the molecular structures of two mutants in the fibrils are similar. Although, EM images and H/D exchange address the structural arrangement at different level, they collectively suggest that familiar mutation impacts on ultrastructural arrangement of protofilaments rather than molecular structure of single protein. Therefore, at the end, several progress still 
need to be made in 1) determining side chain conformation of the key residues in fibril core; 2) determining the $\mathrm{N}$-terminal conformation of $\alpha \mathrm{S}$ in fibril sample and map the potential contacts between $\mathrm{N}$-terminal and fibril core region of $\alpha \mathrm{S}$ since $\mathrm{N}$-terminus of $\alpha \mathrm{S}$ play an important role in modulating fibril formation; 3) clearly identifying the polymorphism existing in the fibrils.

\subsubsection{Structural relationship of $\alpha \mathrm{S}$ fibrils and oligomers and the prospective for structural study of $\alpha \mathrm{S}$ oligomers}

\section{Structural correlation between $\alpha \mathrm{S}$ fibrils and oligomers}

As discussed above, $\mathrm{N}$-terminus of $\alpha \mathrm{S}$ potently act as a role in promoting the fibril formation as the tight interplay between it and fibril core has been implied by structural analysis and aggregation kinetics. And, vice versa, $\alpha \mathrm{S} \mathrm{N}$-terminus is important for the fibrillization and might reduce the oligomeric species by assembling them into fibrils, which accordingly the N-terminal truncation led to longer lag phase (Zibaee et al, 2007). Therefore, it's more likely that N-terminus of $\alpha \mathrm{S}$ is capable to regulate fibril formation of $\alpha \mathrm{S}$.

Low resolution model of $\alpha \mathrm{S}$ oligomers and fibrils indicates that on-pathway oligomers of $\alpha \mathrm{S}$ are the building blocks for $\alpha \mathrm{S}$ fibrils (Giehm et al, 2011a). Our H/D exchange data for the oligomers also tentatively indicated the residues involved in the structured regions of fibril core could show certain degree of protection in the oligomer sample. Similar to this observation on the $\alpha \mathrm{S}$ oligomers, recent progress in structural study of $A \beta$ aggregates collectively by solution NMR and ssNMR revealed that there is close structural relationship amongst $\mathrm{A} \beta$ oligomers, protofilaments and mature fibrils (Fawzi et al, 2011; Lopez Del Amo et al, 2012; Scheidt et al, 2012). Therefore, there might be correlation of $\alpha \mathrm{S}$ oligomer conformation and fibril structure as well. However, until now, there is no clear model to define the details that how oligomers are converted into fibrils. Particularly, the lack of structural information of $\alpha \mathrm{S}$ oligomers at atomic resolution largely hampers to set reasonable link between oligomers and fibrils. In our $\mathrm{H} / \mathrm{D}$ exchange experiment which was optimized for the compounds-stabilized $\alpha \mathrm{S}$ oligomers, one major obstacle is presence of the oligomers as a mixture with $\alpha \mathrm{S}$ 
monomers and thus hard to be purified, concentrated. At the same time, the polymorphisms of the oligomers present in terms of size distribution and structural heterogeneity. Both characters render our experiment with lower sensitivity specific to the oligomers and experimental design.

\section{Prospective for structural study of $\alpha \mathrm{S}$ oligomers}

As the predominant advantage of NMR is revealing the atomic resolution, probing $\alpha \mathrm{S}$ oligomer conformation with at least residue-specific resolution is still of the core tasks pursued in the future research. Based on the experience I attained for structural study of $\alpha \mathrm{S}$ oligomers, several envisions would be made for the following-up studies. 1) Further optimization of $\mathrm{H} / \mathrm{D}$ exchange for the oligomers by searching proper solution conditions and time span for forward exchange period and improving data processing to enhance data sensitivity for the data fraction that encods information of the oligomer conformation. 2) Performing the relaxation dispersion experiments to catch wider time scale dynamics involved in conformational exchange between $\alpha \mathrm{S}$ oligomers and monomers.

Alternatively, other methods would also be of options to access this end. Considering the relative lower population of the oligomers in the mixture of monomers and oligomers, reasonable purification and separation for oligomers are still desirable. Lashuel's group purified $\alpha \mathrm{S}$ oligomers by Superose 6 column of size exclusion chromatography and obtained homogeneous, stable and off-pathway oligomers with size of $10 \sim 14 \mathrm{mer}$ and diameter of $\sim 21 \mathrm{~nm}$ (Hinault et al, 2010). Another feasible approach is to generate oligomers from mature fibrils. $\alpha \mathrm{S}$ fibrils can be reverted back to oligomers through cold-dissociation approach, which was capable to reach $\sim 70 \%$ of total amount staying at oligomeric state under super-cooling condition (Kim et al, 2009; Kim et al, 2008). Moreover, other compounds such as Dopamine (Cappai et al, 2005), EGCG (Ehrnhoefer et al, 2008) which were able to inhibit fibril formation and stabilize $\alpha \mathrm{S}$ oligomers can also be the choices to generate $\alpha \mathrm{S}$ oligomers for structural analysis. 
With optimal sample condition, the following issue is to choose experimental strategy of NMR spectroscopy to characterize conformation of $\alpha \mathrm{S}$ oligomers. In recent years, Clore group characterize amyloid- $\beta$ oligomers by applying selective saturation oligomer-bound monomer together with comparison of apparent $R_{2}$ values between the bound and free monomers. Thereby chemical exchange process up to 1 second timescale between observable monomers and NMR-invisible oligomers can be mapped and residue-specific dynamics containing oligomer conformation can be probed (Fawzi et al, 2011; Fawzi et al, 2010; Fawzi et al, 2012). This is an option to test our oligomer sample. But, the difference for $A \beta$ and $\alpha S$ should be noticed. $A \beta$ is a short peptide with high hydrophobicity, however $\alpha \mathrm{S}$ comprises not only hydrophobic region i.e. NAC but also amphiphilic N-terminal and acidic C-terminal which effectively modulate the oligomerization and fibrillization. Thus, the association of $\alpha \mathrm{S}$ monomers to the oligomers is likely not tight as $A \beta$. Hence kinetics of the chemical exchange process between $\alpha \mathrm{S}$ monomers and oligomers could largely be different with the $\mathrm{A} \beta$ case, which might be out the time scale of $\sim 10 \mathrm{~ms}-1 \mathrm{~s}$ allowing to be detected by the method of Clore (Fawzi et al, 2012).

Complementary to this method, relaxation dispersion as mentioned is one option to describe the dynamics within a wider timescale $(50 \mu \mathrm{s}-10 \mathrm{~ms})$ (Ishima \& Torchia, 2006). Another choice is paramagnetic relaxation enhancement (PRE) to detect the transient complex formation within time scale 250-500 $\mu$ s (Clore \& Iwahara, 2009). Intermolecular PRE was already been detected between N-terminal of one $\alpha \mathrm{S}$ and $\mathrm{C}$ terminal of another $\alpha \mathrm{S}$ in concentration-dependent manner (Wu \& Baum). The intermolecular PRE has been identified by placing paramagnetic tag MTSL at A19C and $\mathrm{G} 132 \mathrm{C}$ of $\alpha \mathrm{S}$, which ${ }^{15} \mathrm{~N}$-labelled $\alpha \mathrm{S}$ mixed with unlabeled $\alpha \mathrm{S}$ with MTSL tag. A position-dependence of this intermolecular PRE has also been indicated by less significant effect for MTSL at A90C position since it's located at the distal side of the interface of intermolecular contact. Recently, the groups of Klenerman and Dobson successfully accessed the structural information of $\alpha \mathrm{S}$ oligomers and identified the conversation between oligomers of different size by placing fluorescence tag at the $90^{\text {th }}$ residue (Cremades et al, 2012). Therefore, it's likely to detect dynamics of chemical exchange between $\alpha \mathrm{S}$ monomers and oligomers by placing paramagnetic tag at proper 
position. For instance, placing MTSL tag at A90C of $\alpha \mathrm{S}$ would not cause significant PRE at neighboring $\alpha \mathrm{S}$ monomers. But, after the preliminary aggregation for mixture of

${ }^{14} \mathrm{~N}-\mathrm{A} 90 \mathrm{C} \alpha \mathrm{S}$ with MTSL and ${ }^{15} \mathrm{~N}-\alpha \mathrm{S}$, the hydrophobic segments of $\alpha \mathrm{S}$ would get close to neighboring $\alpha \mathrm{S}$ during oligomerization and ${ }^{15} \mathrm{~N}-\alpha \mathrm{S}$ would feel the PRE from neighboring ${ }^{14} \mathrm{~N}-\mathrm{A} 90 \mathrm{C} \alpha \mathrm{S}$ with MTSL within oligomers. If free $\alpha \mathrm{S}$ monomers are experiencing intensive chemical exchange with oligomer-bound monomers, there would be possibility that the PRE effect on bound $\alpha \mathrm{S}$ is detectable after it quickly dissociates from oligomers assuming the time scale of this process fits to detect the encoded PRE effects.

\subsection{Structural and functional importance of alpha-Synuclein C- terminus}

The C-terminus of $\alpha \mathrm{S}$ (98-140) displays distinct structural properties and functional significance within the scope of this dissertation. Therefore, herein a brief summary is exerted by linking the roles of $\alpha \mathrm{S}$ C-terminal defined in different topics of this dissertation. The further implications respecting to the previously less-highlighted features of the C-terminal thereby would be proposed.

\section{Structural reasons for the hyperphosphorylation at Ser129 of $\alpha S$}

In the first section, the phosphorylation of $\alpha \mathrm{S}$, we studied the Ser phosphorylation at Ser87 and Ser129. Kinetic studies of the phosphorylation by both CKI and PLKs points out that the 3 familiar mutations of $\alpha \mathrm{S}$ didn't alter the phosphorylation kinetics of Ser87, but slightly modified the kinetics of Ser129. Comparing with CKI, the PLK family kinases phosphorylated $\alpha$ S specifically at Ser129, particularly PLK2 and PLK3 display high efficiency in phosphorylation kinetics both in vitro and in vivo. Furthermore, we performed the aggregation assays for the phosphorylated-Ser129 $\alpha \mathrm{S}$ and for the two Ser-to-Glu mutants that mimic the Ser129 phosphorylation. Our results indicated that the aggregation propensity of $\alpha \mathrm{S}$ was not significantly modified by the phosphorylation at Ser129, drastically in contrast to the aggregation of $\alpha \mathrm{S}$ with the Ser87 phosphorylation (Paleologou et al). The related 
evidences also point out that PLKs are capable of phosphorylating the $\alpha \mathrm{S}$ fibrils at Ser129 (Mbefo et al).

Linking to our H/D exchange data for the fibrils of the two familiar mutants of $\alpha \mathrm{S}, \mathrm{C}$-terminal of $\alpha \mathrm{S}$ is a special region that doesn't participate in the fibril formation and maintains in highly disordered conformation in both monomers and fibrils. This structural feature can address that why approximately $90 \% \alpha \mathrm{S}$ was identified with the Ser129 phosphorylation in the Lewy body (Fujiwara et al, 2002), which is because the $\alpha \mathrm{S} C$-terminal can be readily accessed and recognized by the kinases at both monomeric and fibrillar states. Furthermore, the fact that the post-translational modifications are enriched in C-terminal region (Oueslati et al, 2010b) might be as a consequence of the evolution, where the probability of the posttranslational modifications was maximized for $\alpha \mathrm{S}$ as the sites located in the C-terminal which could be readily accessed even for $\alpha \mathrm{S}$ fibrils.

\section{C-terminus of $\alpha \mathrm{S}$ is important in the protein-protein interactions}

The dominant negative charges at the C-terminus of $\alpha \mathrm{S}$ are recognized to be important for stabilizing monomer conformation and modulating the fibril formation (Levitan et al, 2011). Our interaction studies for $\alpha \mathrm{S}$ and the Rab proteins provide a solid case to understand the role of C-terminal charges in modulating the protein-protein interactions. For instance, Rab8a performs a polyamines-like manner (Fernandez et al, 2004) to interact with $\alpha \mathrm{S}$ C-terminal mainly through the electrostatic interaction. Moreover, it's reported that PLKs possess dominantly positive net-charge over the whole proteins (PLK1: +15, PLK2: +19, PLK3: +15.5, PLK4: +8) (Johnson et al, 2007). At the same time, as described in section 4.1, PLK1 to -3 have a preference for substrates in which at least one of the three residues flanking the serine residue is negatively charged. These two points might shed light to the phosphorylation specificity of PLKs to the C-terminal Ser129 but not the NAC Ser87.

Except the positively charged C-terminus of Rab8a, its folded part can also bind to $\alpha \mathrm{S}$ C-terminus in conformational preference mechanism that Rab8a-GDP displays a stronger affinity than Rab8a-GppNHp binding to $\alpha \mathrm{S}$. This indicates that $\alpha \mathrm{S}$ prefers to bind to GDP-state Rab8a and can be attributed to significant nucleotide-dependence of 
Rab-GTPases conformation, particularly on the Switch regions (Itzen \& Goody, 2011; Lee et al, 2009). Thus, this observation extends our understanding that $\alpha \mathrm{S} C$-terminus binds to other proteins not only upon the charges but also the structural preference.

\section{The role of $\alpha \mathrm{S} \mathrm{C}$-terminus in modulating the aggregation}

Due to the binding of Rab8a to $\alpha \mathrm{S}$ C-terminus, the aggregation of $\alpha \mathrm{S}$ was improved by the presence of Rab8a in a polyamine-like manner that the lag phase was significantly shortened and fibril formation was also enhanced (Fernandez et al, 2004). The same effects have been achieved previously by C-terminal truncation of $\alpha \mathrm{S}$ (Hoyer et al, 2004; Kim et al, 2002; Levitan et al, 2011), which is in contrast to that the N-terminal truncation would increase lag phase of aggregation (Zibaee et al, 2007). Taken together, these evidences collectively suggest that the $\alpha \mathrm{S}$ C-terminus potentially plays a role in modulating the $\alpha \mathrm{S}$ oligomerization. 


\section{$5 \quad$ Summary and outlook}

In this study, we investigated the serine phosphorylation of $\alpha \mathrm{S}$, interactions of $\alpha \mathrm{S}$ with three Rab-GTPases, and the structure of $\alpha \mathrm{S}$ aggregates.

\section{Summary}

1. Serine phosphorylation

(1) Three familial mutations A30P, E46K, and A53T slightly affect the phosphorylation kinetics of Ser129, but do not affect that of Ser87.

(2) PLKs selectively and efficiently phosphorylate $\alpha \mathrm{S}$ at Ser129. PLK3 fully phosphorylate $\alpha \mathrm{S}$ at Ser129 within 2.5 hours under our assay conditions.

(3) Phosphorylation of Ser129 does not affect $\alpha \mathrm{S}$ aggregation.

2. Interactions of $\alpha \mathrm{S}$ with three Rab-GTPases

(1) Rab8a at both GDP- and GppNHp-bound states binds to monomeric $\alpha \mathrm{S}$ at its C-terminus in solution.

(2) Both Rab8a- $\delta \mathrm{C}$ and the C-terminal peptide of Rab8a bind to $\alpha \mathrm{S} C$ terminus as well.

(3) Rab8a(GDP) binds to $\alpha \mathrm{S}$ more strongly than Rab8a(GppNHp) and this is reproduced by $\operatorname{Rab} 8 \mathrm{a}-\delta \mathrm{C}$.

(4) The Switch I region and the C-terminal loop of Rab8a are two major binding sites for $\alpha \mathrm{S}$.

(5) Both Rab8a and its C-terminal peptide can enhance $\alpha \mathrm{S}$ aggregation.

(6) Rab1b and Rab3a do not bind to monomeric $\alpha \mathrm{S}$, but they enhance $\alpha \mathrm{S}$ aggregation. 
3. Structure of $\alpha \mathrm{S}$ aggregates

(1) $\mathrm{H} / \mathrm{D}$ exchange for fibrils of E46K and $\mathrm{A} 53 \mathrm{~T} \alpha \mathrm{S}$ indicates a conserved fibril core.

(2) $H / D$ exchange for the compounds-stabilized oligomers of $\alpha S$ suggests that these oligomers do not contain large amount of hydrogen-bonded regions like $\alpha \mathrm{S}$ fibrils.

\section{Outlook}

To further characterize the comprehensive interplays between $\alpha \mathrm{S}$ and the Rab proteins, we propose to implement:

1) $\alpha \mathrm{S}$ aggregation assays in the presence of the C-terminal peptides of Rab1b, Rab3a and Rab3a, to clarify the role of each fragment of the protein in modulating aggregation;

2) $\alpha \mathrm{S}$ aggregation assays with $\operatorname{Rab} 8 \mathrm{a}-\delta \mathrm{C}$ to exactly determine its role in aggregation modulation;

3) NMR titration of Rab8a and S129D $\alpha$ S to evaluate the effect of the Ser129 phosphorylation on molecular interaction;

4) aggregation assays of phosphorylated Ser129 $\alpha \mathrm{S}$ in the presence of Rab8a;

5) related in vivo assays to study the $\alpha \mathrm{S}-\mathrm{Rab} 8 \mathrm{a}$ co-localization, the aggregation of $\alpha \mathrm{S}$ in the presence of Rab8a and the impacts of Ser129 phosphorylation on the interplay between $\alpha \mathrm{S}$ and Rab8a (undertaken in Prof. Dr. Tiago F. Outeiro's lab);

6) interaction study for $\alpha \mathrm{S}$ oligomers and the Rab proteions. 


\section{References}

Abeliovich A, Schmitz Y, Farinas I, Choi-Lundberg D, Ho WH, Castillo PE, Shinsky N, Verdugo JM, Armanini M, Ryan A, Hynes M, Phillips H, Sulzer D, Rosenthal A (2000) Mice lacking alpha-synuclein display functional deficits in the nigrostriatal dopamine system. Neuron 25(1): 239-252

Anderson JP, Walker DE, Goldstein JM, de Laat R, Banducci K, Caccavello RJ, Barbour R, Huang J, Kling K, Lee M, Diep L, Keim PS, Shen X, Chataway T, Schlossmacher MG, Seubert P, Schenk D, Sinha S, Gai WP, Chilcote TJ (2006) Phosphorylation of Ser-129 is the dominant pathological modification of alphasynuclein in familial and sporadic Lewy body disease. The Journal of biological chemistry 281(40): 29739-29752

Angot E, Steiner JA, Hansen C, Li JY, Brundin P (2010) Are synucleinopathies prionlike disorders? Lancet neurology 9(11): 1128-1138

Artimo P, Jonnalagedda M, Arnold K, Baratin D, Csardi G, de Castro E, Duvaud S, Flegel V, Fortier A, Gasteiger E, Grosdidier A, Hernandez C, Ioannidis V, Kuznetsov D, Liechti R, Moretti S, Mostaguir K, Redaschi N, Rossier G, Xenarios I, Stockinger H (2012) ExPASy: SIB bioinformatics resource portal. Nucleic acids research 40(W1): W597-W603

Auluck PK, Caraveo G, Lindquist S alpha-Synuclein: membrane interactions and toxicity in Parkinson's disease. Annual review of cell and developmental biology 26: 211-233

Auluck PK, Caraveo G, Lindquist S (2010) alpha-Synuclein: membrane interactions and toxicity in Parkinson's disease. Annual review of cell and developmental biology 26: 211-233

Azeredo da Silveira S, Schneider BL, Cifuentes-Diaz C, Sage D, Abbas-Terki T, Iwatsubo T, Unser M, Aebischer P (2009) Phosphorylation does not prompt, nor prevent, the formation of alpha-synuclein toxic species in a rat model of Parkinson's disease. Human molecular genetics 18(5): 872-887

Bartels T, Choi JG, Selkoe DJ (2011) alpha-Synuclein occurs physiologically as a helically folded tetramer that resists aggregation. Nature 477(7362): 107-110

Bax A, Grzesiek S (1993) Methodological Advances in Protein Nmr. Accounts of Chemical Research 26(4): 131-138 
Ben Gedalya T, Loeb V, Israeli E, Altschuler Y, Selkoe DJ, Sharon R (2009) Alphasynuclein and polyunsaturated fatty acids promote clathrin-mediated endocytosis and synaptic vesicle recycling. Traffic (Copenhagen, Denmark) 10(2): 218-234

Bertoncini CW, Fernandez CO, Griesinger C, Jovin TM, Zweckstetter M (2005a) Familial mutants of alpha-synuclein with increased neurotoxicity have a destabilized conformation. The Journal of biological chemistry 280(35): 30649-30652

Bertoncini CW, Jung YS, Fernandez CO, Hoyer W, Griesinger C, Jovin TM, Zweckstetter M (2005b) Release of long-range tertiary interactions potentiates aggregation of natively unstructured alpha-synuclein. Proceedings of the National Academy of Sciences of the United States of America 102(5): 1430-1435

Bisaglia M, Mammi S, Bubacco L (2009) Structural insights on physiological functions and pathological effects of alpha-synuclein. Faseb $J$ 23(2): 329-340

Bleimling N, Alexandrov K, Goody R, Itzen A (2009) Chaperone-assisted production of active human Rab8A GTPase in Escherichia coli. Protein Expr Purif 65(2): 190-195

Bodner CR, Dobson CM, Bax A (2009) Multiple tight phospholipid-binding modes of alpha-synuclein revealed by solution NMR spectroscopy. Journal of molecular biology 390(4): 775-790

Bodner CR, Maltsev AS, Dobson CM, Bax A (2010) Differential phospholipid binding of alpha-synuclein variants implicated in Parkinson's disease revealed by solution NMR spectroscopy. Biochemistry 49(5): 862-871

Braak H, Del Tredici K, Bratzke H, Hamm-Clement J, Sandmann-Keil D, Rub U (2002) Staging of the intracerebral inclusion body pathology associated with idiopathic Parkinson's disease (preclinical and clinical stages). Journal of neurology 249 Suppl 3: III/1-5

Braithwaite SP, Stock JB, Mouradian MM alpha-Synuclein phosphorylation as a therapeutic target in Parkinson's disease. Reviews in the neurosciences 23(2): 191-198

Braithwaite SP, Stock JB, Mouradian MM (2012) alpha-Synuclein phosphorylation as a therapeutic target in Parkinson's disease. Reviews in the neurosciences 23(2): 191-198

Brundin P, Melki R, Kopito $\mathrm{R}$ Prion-like transmission of protein aggregates in neurodegenerative diseases. Nature reviews 11(4): 301-307 
Brundin P, Melki R, Kopito R (2010) Prion-like transmission of protein aggregates in neurodegenerative diseases. Nature reviews 11(4): 301-307

Brundin P, Olsson R (2011) Can alpha-synuclein be targeted in novel therapies for Parkinson's disease? Expert review of neurotherapeutics 11(7): 917-919

Bucci C, Chiariello M, Lattero D, Maiorano M, Bruni CB (1999) Interaction cloning and characterization of the cDNA encoding the human prenylated rab acceptor (PRA1). Biochemical and biophysical research communications 258(3): 657-662

Bussell R, Jr., Eliezer D (2001) Residual structure and dynamics in Parkinson's diseaseassociated mutants of alpha-synuclein. J Biol Chem 276(49): 45996-46003

Butterfield SM, Lashuel HA Amyloidogenic protein-membrane interactions: mechanistic insight from model systems. Angewandte Chemie (International ed 49(33): $5628-5654$

Cabin DE, Shimazu K, Murphy D, Cole NB, Gottschalk W, McIlwain KL, Orrison B, Chen A, Ellis CE, Paylor R, Lu B, Nussbaum RL (2002) Synaptic vesicle depletion correlates with attenuated synaptic responses to prolonged repetitive stimulation in mice lacking alpha-synuclein. J Neurosci 22(20): 8797-8807

Cappai R, Leck SL, Tew DJ, Williamson NA, Smith DP, Galatis D, Sharples RA, Curtain CC, Ali FE, Cherny RA, Culvenor JG, Bottomley SP, Masters CL, Barnham KJ, Hill AF (2005) Dopamine promotes alpha-synuclein aggregation into SDS-resistant soluble oligomers via a distinct folding pathway. Faseb J 19(10): 1377-1379

Carulla N, Zhou M, Giralt E, Robinson CV, Dobson CM (2010) Structure and intermolecular dynamics of aggregates populated during amyloid fibril formation studied by hydrogen/deuterium exchange. Accounts of chemical research 43(8): 10721079

Caughey B, Lansbury PT (2003) Protofibrils, pores, fibrils, and neurodegeneration: separating the responsible protein aggregates from the innocent bystanders. Annual review of neuroscience 26: $267-298$

Cavanagh J, Fairbrother W, Palmer III A, Rance M, Skelton N. (2007) Protein NMR Spectroscopy (Second Edition) Elsevier Inc. , p. 757.

Chandra S, Gallardo G, Fernandez-Chacon R, Schluter OM, Sudhof TC (2005) Alphasynuclein cooperates with CSPalpha in preventing neurodegeneration. Cell 123(3): 383396 
Chaudhuri KR, Healy DG, Schapira AH (2006) Non-motor symptoms of Parkinson's disease: diagnosis and management. Lancet neurology 5(3): 235-245

Chen L, Feany MB (2005) Alpha-synuclein phosphorylation controls neurotoxicity and inclusion formation in a Drosophila model of Parkinson disease. Nature neuroscience 8(5): $657-663$

Chen L, Periquet M, Wang X, Negro A, McLean PJ, Hyman BT, Feany MB (2009) Tyrosine and serine phosphorylation of alpha-synuclein have opposing effects on neurotoxicity and soluble oligomer formation. The Journal of clinical investigation 119(11): 3257-3265

Chen M, Margittai M, Chen J, Langen R (2007) Investigation of alpha-synuclein fibril structure by site-directed spin labeling. The Journal of biological chemistry 282(34): 24970-24979

Cho MK, Kim HY, Bernado P, Fernandez CO, Blackledge M, Zweckstetter M (2007) Amino acid bulkiness defines the local conformations and dynamics of natively unfolded alpha-synuclein and tau. Journal of the American Chemical Society 129(11): 3032-3033

Cho MK, Kim HY, Fernandez CO, Becker S, Zweckstetter M (2011) Conserved core of amyloid fibrils of wild type and A30P mutant alpha-synuclein. Protein Sci 20(2): 387395

Chung CY, Koprich JB, Hallett PJ, Isacson O (2009) Functional enhancement and protection of dopaminergic terminals by RAB3B overexpression. Proceedings of the National Academy of Sciences of the United States of America 106(52): 22474-22479

Clore GM, Iwahara J (2009) Theory, practice, and applications of paramagnetic relaxation enhancement for the characterization of transient low-population states of biological macromolecules and their complexes. Chemical reviews 109(9): 4108-4139

Comellas G, Lemkau LR, Nieuwkoop AJ, Kloepper KD, Ladror DT, Ebisu R, Woods WS, Lipton AS, George JM, Rienstra CM (2011) Structured regions of alpha-synuclein fibrils include the early-onset Parkinson's disease mutation sites. Journal of molecular biology 411(4): 881-895

Conway KA, Harper JD, Lansbury PT (1998) Accelerated in vitro fibril formation by a mutant alpha-synuclein linked to early-onset Parkinson disease. Nature medicine 4(11): $1318-1320$

Conway KA, Lee SJ, Rochet JC, Ding TT, Williamson RE, Lansbury PT, Jr. (2000) Acceleration of oligomerization, not fibrillization, is a shared property of both alpha- 
synuclein mutations linked to early-onset Parkinson's disease: implications for pathogenesis and therapy. Proceedings of the National Academy of Sciences of the United States of America 97(2): 571-576

Cookson MR (2009) alpha-Synuclein and neuronal cell death. Molecular neurodegeneration 4: 9

Cookson MR, van der Brug M (2008) Cell systems and the toxic mechanism(s) of alpha-synuclein. Experimental neurology 209(1): 5-11

Cooper AA, Gitler AD, Cashikar A, Haynes CM, Hill KJ, Bhullar B, Liu K, Xu K, Strathearn KE, Liu F, Cao S, Caldwell KA, Caldwell GA, Marsischky G, Kolodner RD, Labaer J, Rochet JC, Bonini NM, Lindquist S (2006) Alpha-synuclein blocks ER-Golgi traffic and Rabl rescues neuron loss in Parkinson's models. Science (New York, NY 313(5785): 324-328

Craik DJ, Wilce JA (1997) Studies of protein-ligand interactions by NMR. Methods Mol Biol 60: 195-232

Cremades N, Cohen SI, Deas E, Abramov AY, Chen AY, Orte A, Sandal M, Clarke RW, Dunne P, Aprile FA, Bertoncini CW, Wood NW, Knowles TP, Dobson CM, Klenerman D (2012) Direct observation of the interconversion of normal and toxic forms of alpha-synuclein. Cell 149(5): 1048-1059

Dalfo E, Barrachina M, Rosa JL, Ambrosio S, Ferrer I (2004a) Abnormal alphasynuclein interactions with rab3a and rabphilin in diffuse Lewy body disease. Neurobiology of disease 16(1): 92-97

Dalfo E, Gomez-Isla T, Rosa JL, Nieto Bodelon M, Cuadrado Tejedor M, Barrachina M, Ambrosio S, Ferrer I (2004b) Abnormal alpha-synuclein interactions with Rab proteins in alpha-synuclein A30P transgenic mice. Journal of neuropathology and experimental neurology 63(4): 302-313

Davidson WS, Jonas A, Clayton DF, George JM (1998) Stabilization of alpha-synuclein secondary structure upon binding to synthetic membranes. The Journal of biological chemistry 273(16): 9443-9449

de Rijk MC, Launer LJ, Berger K, Breteler MM, Dartigues JF, Baldereschi M, Fratiglioni L, Lobo A, Martinez-Lage J, Trenkwalder C, Hofman A (2000) Prevalence of Parkinson's disease in Europe: A collaborative study of population-based cohorts. Neurologic Diseases in the Elderly Research Group. Neurology 54(11 Suppl 5): S21-23 
Dedmon MM, Christodoulou J, Wilson MR, Dobson CM (2005a) Heat shock protein 70 inhibits alpha-synuclein fibril formation via preferential binding to prefibrillar species. Journal of Biological Chemistry 280(15): 14733-14740

Dedmon MM, Christodoulou J, Wilson MR, Dobson CM (2005b) Heat shock protein 70 inhibits alpha-synuclein fibril formation via preferential binding to prefibrillar species. The Journal of biological chemistry 280(15): 14733-14740

Dedmon MM, Lindorff-Larsen K, Christodoulou J, Vendruscolo M, Dobson CM (2005c) Mapping long-range interactions in alpha-synuclein using spin-label NMR and ensemble molecular dynamics simulations. Journal of the American Chemical Society 127(2): 476-477

Del Mar C, Greenbaum EA, Mayne L, Englander SW, Woods VL, Jr. (2005) Structure and properties of alpha-synuclein and other amyloids determined at the amino acid level. Proceedings of the National Academy of Sciences of the United States of America 102(43): 15477-15482

Di Giovanni S, Eleuteri S, Paleologou KE, Yin G, Zweckstetter M, Carrupt PA, Lashuel HA (2010) Entacapone and tolcapone, two catechol O-methyltransferase inhibitors, block fibril formation of alpha-synuclein and beta-amyloid and protect against amyloidinduced toxicity. The Journal of biological chemistry 285(20): 14941-14954

Dolgin E (2012) First therapy targeting Parkinson's proteins enters clinical trials. Nature medicine 18(7): 992-993

Dumas JJ, Zhu Z, Connolly JL, Lambright DG (1999) Structural basis of activation and GTP hydrolysis in Rab proteins. Structure 7(4): 413-423

Dyson HJ, Wright PE (2004) Unfolded proteins and protein folding studied by NMR. Chemical Reviews 104(8): 3607-3622

Dyson HJ, Wright PE (2005) Intrinsically unstructured proteins and their functions. Nature reviews 6(3): 197-208

Ehrnhoefer DE, Bieschke J, Boeddrich A, Herbst M, Masino L, Lurz R, Engemann S, Pastore A, Wanker EE (2008) EGCG redirects amyloidogenic polypeptides into unstructured, off-pathway oligomers. Nature structural \& molecular biology 15(6): 558-566

el-Agnaf OM, Irvine GB (2002) Aggregation and neurotoxicity of alpha-synuclein and related peptides. Biochemical Society transactions 30(4): 559-565 
El-Agnaf OM, Jakes R, Curran MD, Middleton D, Ingenito R, Bianchi E, Pessi A, Neill D, Wallace A (1998) Aggregates from mutant and wild-type alpha-synuclein proteins and NAC peptide induce apoptotic cell death in human neuroblastoma cells by formation of beta-sheet and amyloid-like filaments. FEBS letters 440(1-2): 71-75

Exner N, Lutz AK, Haass C, Winklhofer KF (2012) Mitochondrial dysfunction in Parkinson's disease: molecular mechanisms and pathophysiological consequences. The EMBO journal 31(14): 3038-3062

Falsone SF, Kungl AJ, Rek A, Cappai R, Zangger K (2009) The molecular chaperone Hsp90 modulates intermediate steps of amyloid assembly of the Parkinson-related protein alpha-synuclein. The Journal of biological chemistry 284(45): 31190-31199

Fauvet B, Fares MB, Samuel F, Dikiy I, Tandon A, Eliezer D, Lashuel HA (2012a) Characterization of Semisynthetic and Naturally Nalpha-Acetylated alpha-Synuclein in Vitro and in Intact Cells: IMPLICATIONS FOR AGGREGATION AND CELLULAR PROPERTIES OF alpha-SYNUCLEIN. The Journal of biological chemistry 287(34): 28243-28262

Fauvet B, Mbefo MK, Fares MB, Desobry C, Michael S, Ardah MT, Tsika E, Coune P, Prudent M, Lion N, Eliezer D, Moore DJ, Schneider B, Aebischer P, El-Agnaf OM, Masliah E, Lashuel HA (2012b) alpha-Synuclein in Central Nervous System and from Erythrocytes, Mammalian Cells, and Escherichia coli Exists Predominantly as Disordered Monomer. Journal of Biological Chemistry 287(19): 15345-15364

Fawzi NL, Ying J, Ghirlando R, Torchia DA, Clore GM (2011) Atomic-resolution dynamics on the surface of amyloid-beta protofibrils probed by solution NMR. Nature 480(7376): 268-272

Fawzi NL, Ying J, Torchia DA, Clore GM (2010) Kinetics of amyloid beta monomerto-oligomer exchange by NMR relaxation. Journal of the American Chemical Society 132(29): 9948-9951

Fawzi NL, Ying J, Torchia DA, Clore GM (2012) Probing exchange kinetics and atomic resolution dynamics in high-molecular-weight complexes using dark-state exchange saturation transfer NMR spectroscopy. Nature protocols 7(8): 1523-1533

Fernandez CO, Hoyer W, Zweckstetter M, Jares-Erijman EA, Subramaniam V, Griesinger C, Jovin TM (2004) NMR of alpha-synuclein-polyamine complexes elucidates the mechanism and kinetics of induced aggregation. Embo $J$ 23(10): 20392046 
Figueroa C, Taylor J, Vojtek AB (2001) Prenylated Rab acceptor protein is a receptor for prenylated small GTPases. The Journal of biological chemistry 276(30): 2821928225

Fink AL (2006) The aggregation and fibrillation of alpha-synuclein. Accounts of Chemical Research 39(9): 628-634

Fredenburg RA, Rospigliosi C, Meray RK, Kessler JC, Lashuel HA, Eliezer D, Lansbury PT, Jr. (2007) The impact of the E46K mutation on the properties of alphasynuclein in its monomeric and oligomeric states. Biochemistry 46(24): 7107-7118

Fujiwara H, Hasegawa M, Dohmae N, Kawashima A, Masliah E, Goldberg MS, Shen J, Takio K, Iwatsubo T (2002) alpha-Synuclein is phosphorylated in synucleinopathy lesions. Nature cell biology 4(2): 160-164

Gao G, Williams JG, Campbell SL (2004) Protein-protein interaction analysis by nuclear magnetic resonance spectroscopy. Methods Mol Biol 261: 79-92

Gath J, Habenstein B, Bousset L, Melki R, Meier BH, Bockmann A (2012) Solid-state NMR sequential assignments of alpha-synuclein. Biomolecular NMR assignments 6(1): $51-55$

Giasson BI, Duda JE, Murray IV, Chen Q, Souza JM, Hurtig HI, Ischiropoulos H, Trojanowski JQ, Lee VM (2000) Oxidative damage linked to neurodegeneration by selective alpha-synuclein nitration in synucleinopathy lesions. Science (New York, NY 290(5493): 985-989

Giasson BI, Murray IV, Trojanowski JQ, Lee VM (2001) A hydrophobic stretch of 12 amino acid residues in the middle of alpha-synuclein is essential for filament assembly. The Journal of biological chemistry 276(4): 2380-2386

Giehm L, Svergun DI, Otzen DE, Vestergaard B (2011a) Low-resolution structure of a vesicle disrupting \&alpha;-synuclein oligomer that accumulates during fibrillation. Proceedings of the National Academy of Sciences of the United States of America 108(8): 3246-3251

Giehm L, Svergun DI, Otzen DE, Vestergaard B (2011b) Low-resolution structure of a vesicle disrupting alpha-synuclein oligomer that accumulates during fibrillation. Proceedings of the National Academy of Sciences of the United States of America 108(8): 3246-3251

Gitler AD, Bevis BJ, Shorter J, Strathearn KE, Hamamichi S, Su LJ, Caldwell KA, Caldwell GA, Rochet JC, McCaffery JM, Barlowe C, Lindquist S (2008) The Parkinson's disease protein alpha-synuclein disrupts cellular Rab homeostasis. 
Proceedings of the National Academy of Sciences of the United States of America 105(1): 145-150

Golbe LI, Di Iorio G, Bonavita V, Miller DC, Duvoisin RC (1990) A large kindred with autosomal dominant Parkinson's disease. Ann Neurol 27(3): 276-282

Gomez-Tortosa E, Newell K, Irizarry MC, Sanders JL, Hyman BT (2000) alphaSynuclein immunoreactivity in dementia with Lewy bodies: morphological staging and comparison with ubiquitin immunostaining. Acta neuropathologica 99(4): 352-357

Gorbatyuk OS, Li S, Sullivan LF, Chen W, Kondrikova G, Manfredsson FP, Mandel RJ, Muzyczka N (2008) The phosphorylation state of Ser-129 in human alpha-synuclein determines neurodegeneration in a rat model of Parkinson disease. Proceedings of the National Academy of Sciences of the United States of America 105(2): 763-768

Gosavi N, Lee HJ, Lee JS, Patel S, Lee SJ (2002) Golgi fragmentation occurs in the cells with prefibrillar alpha-synuclein aggregates and precedes the formation of fibrillar inclusion. The Journal of biological chemistry 277(50): 48984-48992

Greenbaum EA, Graves CL, Mishizen-Eberz AJ, Lupoli MA, Lynch DR, Englander SW, Axelsen PH, Giasson BI (2005) The E46K mutation in alpha-synuclein increases amyloid fibril formation. The Journal of biological chemistry 280(9): 7800-7807

Guex N, Peitsch MC (1997) SWISS-MODEL and the Swiss-PdbViewer: an environment for comparative protein modeling. Electrophoresis 18(15): 2714-2723

Halliday GM, Holton JL, Revesz T, Dickson DW (2011) Neuropathology underlying clinical variability in patients with synucleinopathies. Acta neuropathologica 122(2): 187-204

Hansen C, Angot E, Bergstrom AL, Steiner JA, Pieri L, Paul G, Outeiro TF, Melki R, Kallunki P, Fog K, Li JY, Brundin P alpha-Synuclein propagates from mouse brain to grafted dopaminergic neurons and seeds aggregation in cultured human cells. The Journal of clinical investigation 121(2): 715-725

Hardy J (2010) Genetic Analysis of Pathways to Parkinson Disease. Neuron 68(2): 201206

Hardy J, Lewis P, Revesz T, Lees A, Paisan-Ruiz C (2009) The genetics of Parkinson's syndromes: a critical review. Curr Opin Genet Dev 19(3): 254-265

Hartl FU, Bracher A, Hayer-Hartl M (2011) Molecular chaperones in protein folding and proteostasis. Nature 475(7356): 324-332 
Hartl FU, Hayer-Hartl M (2009) Converging concepts of protein folding in vitro and in vivo. Nature structural \& molecular biology 16(6): 574-581

Hawkes CH, Del Tredici K, Braak H (2007) Parkinson's disease: a dual-hit hypothesis. Neuropathology and applied neurobiology 33(6): 599-614

Heise H, Celej MS, Becker S, Riedel D, Pelah A, Kumar A, Jovin TM, Baldus M (2008) Solid-state NMR reveals structural differences between fibrils of wild-type and diseaserelated A53T mutant alpha-synuclein. Journal of molecular biology 380(3): 444-450

Heise H, Hoyer W, Becker S, Andronesi OC, Riedel D, Baldus M (2005) Molecularlevel secondary structure, polymorphism, and dynamics of full-length alpha-synuclein fibrils studied by solid-state NMR. Proceedings of the National Academy of Sciences of the United States of America 102(44): 15871-15876

Hejjaoui M, Butterfield S, Fauvet B, Vercruysse F, Cui J, Dikiy I, Prudent M, Olschewski D, Zhang Y, Eliezer D, Lashuel HA (2012) Elucidating the role of Cterminal post-translational modifications using protein semisynthesis strategies: alphasynuclein phosphorylation at tyrosine 125. Journal of the American Chemical Society 134(11): 5196-5210

Hinault MP, Cuendet AF, Mattoo RU, Mensi M, Dietler G, Lashuel HA, Goloubinoff P (2010) Stable alpha-synuclein oligomers strongly inhibit chaperone activity of the Hsp70 system by weak interactions with J-domain co-chaperones. The Journal of biological chemistry 285(49): 38173-38182

Hou X, Hagemann N, Schoebel S, Blankenfeldt W, Goody RS, Erdmann KS, Itzen A (2011) A structural basis for Lowe syndrome caused by mutations in the Rab-binding domain of OCRL1. Embo J 30(8): 1659-1670

Hoyer W, Antony T, Cherny D, Heim G, Jovin TM, Subramaniam V (2002) Dependence of alpha-synuclein aggregate morphology on solution conditions. Journal of molecular biology 322(2): 383-393

Hoyer W, Cherny D, Subramaniam V, Jovin TM (2004) Impact of the acidic C-terminal region comprising amino acids 109-140 on alpha-synuclein aggregation in vitro. Biochemistry 43(51): 16233-16242

Huang CJ, Cheng H, Hao SF, Zhou H, Zhang XJ, Gao JN, Sun QH, Hu HY, Wang CC (2006) Heat shock protein 70 inhibits alpha-synuclein fibril formation via interactions with diverse intermediates. Journal of molecular biology 364(3): 323-336 
Hutagalung AH, Novick PJ (2011) Role of Rab GTPases in membrane traffic and cell physiology. Physiological reviews 91(1): 119-149

Hutt DM, Da-Silva LF, Chang LH, Prosser DC, Ngsee JK (2000) PRA1 inhibits the extraction of membrane-bound rab GTPase by GDI1. The Journal of biological chemistry 275(24): 18511-18519

Huynh T (2011) The Parkinson's disease market. Nature reviews 10(8): 571-572

Inglis KJ, Chereau D, Brigham EF, Chiou SS, Schobel S, Frigon NL, Yu M, Caccavello RJ, Nelson S, Motter R, Wright S, Chian D, Santiago P, Soriano F, Ramos C, Powell K, Goldstein JM, Babcock M, Yednock T, Bard F, Basi GS, Sham H, Chilcote TJ, McConlogue L, Griswold-Prenner I, Anderson JP (2009) Polo-like kinase 2 (PLK2) phosphorylates alpha-synuclein at serine 129 in central nervous system. The Journal of biological chemistry 284(5): 2598-2602

Ishii A, Nonaka T, Taniguchi S, Saito T, Arai T, Mann D, Iwatsubo T, Hisanaga S, Goedert M, Hasegawa M (2007) Casein kinase 2 is the major enzyme in brain that phosphorylates Ser129 of human alpha-synuclein: Implication for alphasynucleinopathies. FEBS Lett 581(24): 4711-4717

Ishima R, Torchia DA (2006) Accuracy of optimized chemical-exchange parameters derived by fitting CPMG R2 dispersion profiles when R2(0a) not $=\mathrm{R} 2(0 \mathrm{~b})$. Journal of biomolecular NMR 34(4): 209-219

Itzen A, Goody RS (2011) GTPases involved in vesicular trafficking: structures and mechanisms. Seminars in cell \& developmental biology 22(1): 48-56

Jensen MR, Markwick PR, Meier S, Griesinger C, Zweckstetter M, Grzesiek S, Bernado P, Blackledge M (2009) Quantitative determination of the conformational properties of partially folded and intrinsically disordered proteins using NMR dipolar couplings. Structure 17(9): 1169-1185

Johnson CS (1999) Diffusion ordered nuclear magnetic resonance spectroscopy: principles and applications. Progress in Nuclear Magnetic Resonance Spectroscopy 34(3-4): 203-256

Johnson EF, Stewart KD, Woods KW, Giranda VL, Luo Y (2007) Pharmacological and functional comparison of the polo-like kinase family: insight into inhibitor and substrate specificity. Biochemistry 46(33): 9551-9563

Jung YS, Zweckstetter M (2004a) Backbone assignment of proteins with known structure using residual dipolar couplings. Journal of biomolecular NMR 30(1): 25-35 
Jung YS, Zweckstetter M (2004b) Mars -- robust automatic backbone assignment of proteins. Journal of biomolecular NMR 30(1): 11-23

Kamp F, Exner N, Lutz AK, Wender N, Hegermann J, Brunner B, Nuscher B, Bartels T, Giese A, Beyer K, Eimer S, Winklhofer KF, Haass C (2010) Inhibition of mitochondrial fusion by alpha-synuclein is rescued by PINK1, Parkin and DJ-1. The EMBO journal 29(20): 3571-3589

Kang L, Moriarty GM, Woods LA, Ashcroft AE, Radford SE, Baum J (2012) Nterminal acetylation of alpha-synuclein induces increased transient helical propensity and decreased aggregation rates in the intrinsically disordered monomer. Protein Sci 21(7): $911-917$

Karpinar DP, Balija MB, Kugler S, Opazo F, Rezaei-Ghaleh N, Wender N, Kim HY, Taschenberger G, Falkenburger BH, Heise H, Kumar A, Riedel D, Fichtner L, Voigt A, Braus GH, Giller K, Becker S, Herzig A, Baldus M, Jackle H, Eimer S, Schulz JB, Griesinger C, Zweckstetter M (2009) Pre-fibrillar alpha-synuclein variants with impaired beta-structure increase neurotoxicity in Parkinson's disease models. The EMBO journal 28(20): 3256-3268

Kessler JC, Rochet JC, Lansbury PT, Jr. (2003) The N-terminal repeat domain of alphasynuclein inhibits beta-sheet and amyloid fibril formation. Biochemistry 42(3): 672-678

Kim HY, Cho MK, Kumar A, Maier E, Siebenhaar C, Becker S, Fernandez CO, Lashuel HA, Benz R, Lange A, Zweckstetter M (2009) Structural properties of poreforming oligomers of alpha-synuclein. Journal of the American Chemical Society 131(47): 17482-17489

Kim HY, Cho MK, Riedel D, Fernandez CO, Zweckstetter M (2008) Dissociation of amyloid fibrils of alpha-synuclein in supercooled water. Angewandte Chemie (International ed 47(27): 5046-5048

Kim TD, Paik SR, Yang CH (2002) Structural and functional implications of C-terminal regions of alpha-synuclein. Biochemistry 41(46): 13782-13790

Kloepper KD, Hartman KL, Ladror DT, Rienstra CM (2007) Solid-state NMR spectroscopy reveals that water is nonessential to the core structure of alpha-synuclein fibrils. The journal of physical chemistry 111(47): 13353-13356

Kontaxis G, Clore GM, Bax A (2000) Evaluation of cross-correlation effects and measurement of one-bond couplings in proteins with short transverse relaxation times. $J$ Magn Reson 143(1): 184-196 
Kruger R, Kuhn W, Muller T, Woitalla D, Graeber M, Kosel S, Przuntek H, Epplen JT, Schols L, Riess O (1998) Ala30Pro mutation in the gene encoding alpha-synuclein in Parkinson's disease. Nat Genet 18(2): 106-108

Kuwahara T, Tonegawa R, Ito G, Mitani S, Iwatsubo T (2012) Phosphorylation of alpha-synuclein protein at Ser-129 reduces neuronal dysfunction by lowering its membrane binding property in Caenorhabditis elegans. The Journal of biological chemistry 287(10): 7098-7109

Landrieu I, Lacosse L, Leroy A, Wieruszeski JM, Trivelli X, Sillen A, Sibille N, Schwalbe H, Saxena K, Langer T, Lippens G (2006) NMR analysis of a Tau phosphorylation pattern. J Am Chem Soc 128(11): 3575-3583

Lansbury PT, Lashuel HA (2006) A century-old debate on protein aggregation and neurodegeneration enters the clinic. Nature 443(7113): 774-779

Larsen KE, Schmitz Y, Troyer MD, Mosharov E, Dietrich P, Quazi AZ, Savalle M, Nemani V, Chaudhry FA, Edwards RH, Stefanis L, Sulzer D (2006) Alpha-synuclein overexpression in PC12 and chromaffin cells impairs catecholamine release by interfering with a late step in exocytosis. J Neurosci 26(46): 11915-11922

Lashuel HA, Hartley D, Petre BM, Walz T, Lansbury PT, Jr. (2002a) Neurodegenerative disease: amyloid pores from pathogenic mutations. Nature 418(6895): 291

Lashuel HA, Lansbury PT, Jr. (2006) Are amyloid diseases caused by protein aggregates that mimic bacterial pore-forming toxins? Quarterly reviews of biophysics 39(2): $167-201$

Lashuel HA, Petre BM, Wall J, Simon M, Nowak RJ, Walz T, Lansbury PT, Jr. (2002b) Alpha-synuclein, especially the Parkinson's disease-associated mutants, forms pore-like annular and tubular protofibrils. Journal of molecular biology 322(5): 1089-1102

Lee HJ, Kang SJ, Lee K, Im H (2011) Human alpha-synuclein modulates vesicle trafficking through its interaction with prenylated Rab acceptor protein 1. Biochemical and biophysical research communications 412(4): 526-531

Lee MT, Mishra A, Lambright DG (2009) Structural mechanisms for regulation of membrane traffic by rab GTPases. Traffic (Copenhagen, Denmark) 10(10): 1377-1389

Lees AJ, Hardy J, Revesz T (2009) Parkinson's disease. Lancet 373(9680): 2055-2066 
Lemkau LR, Comellas G, Kloepper KD, Woods WS, George JM, Rienstra CM (2012) Mutant Protein A30P alpha-Synuclein Adopts Wild-type Fibril Structure, Despite Slower Fibrillation Kinetics. The Journal of biological chemistry 287(14): 11526-11532

Lescop E, Kern T, Brutscher B (2010) Guidelines for the use of band-selective radiofrequency pulses in hetero-nuclear NMR: example of longitudinal-relaxationenhanced BEST-type 1H-15N correlation experiments. J Magn Reson 203(1): 190-198

LeVine H, 3rd (1999) Quantification of beta-sheet amyloid fibril structures with thioflavin T. Methods in enzymology 309: 274-284

Levitan K, Chereau D, Cohen SI, Knowles TP, Dobson CM, Fink AL, Anderson JP, Goldstein JM, Millhauser GL (2011) Conserved C-terminal charge exerts a profound influence on the aggregation rate of alpha-synuclein. Journal of molecular biology 411(2): 329-333

Li JY, Englund E, Holton JL, Soulet D, Hagell P, Lees AJ, Lashley T, Quinn NP, Rehncrona S, Bjorklund A, Widner H, Revesz T, Lindvall O, Brundin P (2008) Lewy bodies in grafted neurons in subjects with Parkinson's disease suggest host-to-graft disease propagation. Nature medicine 14(5): 501-503

Li W, West N, Colla E, Pletnikova O, Troncoso JC, Marsh L, Dawson TM, Jakala P, Hartmann T, Price DL, Lee MK (2005) Aggregation promoting C-terminal truncation of alpha-synuclein is a normal cellular process and is enhanced by the familial Parkinson's disease-linked mutations. Proceedings of the National Academy of Sciences of the United States of America 102(6): 2162-2167

Lim Y, Kehm VM, Lee EB, Soper JH, Li C, Trojanowski JQ, Lee VM (2011) alphaSyn suppression reverses synaptic and memory defects in a mouse model of dementia with Lewy bodies. J Neurosci 31(27): 10076-10087

Liu CW, Giasson BI, Lewis KA, Lee VM, Demartino GN, Thomas PJ (2005) A precipitating role for truncated alpha-synuclein and the proteasome in alpha-synuclein aggregation: implications for pathogenesis of Parkinson disease. The Journal of biological chemistry 280(24): 22670-22678

Liu J, Zhang JP, Shi M, Quinn T, Bradner J, Beyer R, Chen S, Zhang J (2009) Rab11a and HSP90 regulate recycling of extracellular alpha-synuclein. J Neurosci 29(5): 14801485

Liu S, Ninan I, Antonova I, Battaglia F, Trinchese F, Narasanna A, Kolodilov N, Dauer W, Hawkins RD, Arancio O (2004) alpha-Synuclein produces a long-lasting increase in neurotransmitter release. The EMBO journal 23(22): 4506-4516 
Lo Bianco C, Shorter J, Regulier E, Lashuel H, Iwatsubo T, Lindquist S, Aebischer P (2008) Hsp104 antagonizes alpha-synuclein aggregation and reduces dopaminergic degeneration in a rat model of Parkinson disease. The Journal of clinical investigation 118(9): 3087-3097

Lopez Del Amo JM, Fink U, Dasari M, Grelle G, Wanker EE, Bieschke J, Reif B (2012) Structural Properties of EGCG-Induced, Nontoxic Alzheimer's Disease Abeta Oligomers. Journal of molecular biology 421(4-5): 517-524

Luk KC, Song C, O'Brien P, Stieber A, Branch JR, Brunden KR, Trojanowski JQ, Lee VM (2009) Exogenous alpha-synuclein fibrils seed the formation of Lewy body-like intracellular inclusions in cultured cells. Proceedings of the National Academy of Sciences of the United States of America 106(47): 20051-20056

Lv G, Kumar A, Giller K, Orcellet ML, Riedel D, Fernandez CO, Becker S, Lange A Structural comparison of mouse and human alpha-synuclein amyloid fibrils by solidstate NMR. Journal of molecular biology 420(1-2): 99-111

Madine J, Doig AJ, Middleton DA (2008) Design of an N-methylated peptide inhibitor of alpha-synuclein aggregation guided by solid-state NMR. Journal of the American Chemical Society 130(25): 7873-7881

Madine J, Middleton DA (2009) TARGETING alpha-SYNUCLEIN AGGREGATION FOR PARKINSON'S DISEASE TREATMENT. Drugs of the Future 34(8): 655-663

Maltsev AS, Ying J, Bax A (2012) Impact of N-terminal acetylation of alpha-synuclein on its random coil and lipid binding properties. Biochemistry 51(25): 5004-5013

Martincic I, Peralta ME, Ngsee JK (1997) Isolation and characterization of a dual prenylated Rab and VAMP2 receptor. The Journal of biological chemistry 272(43): 26991-26998

Mbefo MK, Paleologou KE, Boucharaba A, Oueslati A, Schell H, Fournier M, Olschewski D, Yin G, Zweckstetter M, Masliah E, Kahle PJ, Hirling H, Lashuel HA Phosphorylation of synucleins by members of the Polo-like kinase family. The Journal of biological chemistry 285(4): 2807-2822

Mbefo MK, Paleologou KE, Boucharaba A, Oueslati A, Schell H, Fournier M, Olschewski D, Yin G, Zweckstetter M, Masliah E, Kahle PJ, Hirling H, Lashuel HA (2010) Phosphorylation of synucleins by members of the Polo-like kinase family. The Journal of biological chemistry 285(4): 2807-2822

McFarland NR, Fan Z, Xu K, Schwarzschild MA, Feany MB, Hyman BT, McLean PJ (2009) Alpha-synuclein S129 phosphorylation mutants do not alter nigrostriatal toxicity 
in a rat model of Parkinson disease. Journal of neuropathology and experimental neurology 68(5): 515-524

McLean PJ, Ribich S, Hyman BT (2000) Subcellular localization of alpha-synuclein in primary neuronal cultures: effect of missense mutations. Journal of neural transmission (58): 53-63

Milburn MV, Tong L, deVos AM, Brunger A, Yamaizumi Z, Nishimura S, Kim SH (1990) Molecular switch for signal transduction: structural differences between active and inactive forms of protooncogenic ras proteins. Science (New York, NY 247(4945): 939-945

Morris GA, Freeman R (1979) Enhancement of Nuclear Magnetic-Resonance Signals by Polarization Transfer. Journal of the American Chemical Society 101(3): 760-762

Mukrasch MD, Markwick P, Biernat J, Bergen M, Bernado P, Griesinger C, Mandelkow E, Zweckstetter M, Blackledge M (2007) Highly populated turn conformations in natively unfolded tau protein identified from residual dipolar couplings and molecular simulation. Journal of the American Chemical Society 129(16): $5235-5243$

Mulder FAA, Spronk CAEM, Slijper M, Kaptein R, Boelens R (1996) Improved HSQC experiments for the observation of exchange broadened signals. Journal of biomolecular NMR 8(2): 223-228

Muller MP, Peters H, Blumer J, Blankenfeldt W, Goody RS, Itzen A (2010) The Legionella effector protein DrrA AMPylates the membrane traffic regulator Rablb. Science (New York, NY 329(5994): 946-949

Murphy DD, Rueter SM, Trojanowski JQ, Lee VM (2000) Synucleins are developmentally expressed, and alpha-synuclein regulates the size of the presynaptic vesicular pool in primary hippocampal neurons. J Neurosci 20(9): 3214-3220

Nalls MA, Plagnol V, Hernandez DG, Sharma M, Sheerin UM, Saad M, SimonSanchez J, Schulte C, Lesage S, Sveinbjornsdottir S, Stefansson K, Martinez M, Hardy J, Heutink P, Brice A, Gasser T, Singleton AB, Wood NW (2011) Imputation of sequence variants for identification of genetic risks for Parkinson's disease: a metaanalysis of genome-wide association studies. Lancet 377(9766): 641-649

Nonaka T, Iwatsubo T, Hasegawa M (2005) Ubiquitination of alpha-synuclein. Biochemistry 44(1): 361-368

Notredame C, Higgins DG, Heringa J (2000) T-Coffee: A novel method for fast and accurate multiple sequence alignment. Journal of molecular biology 302(1): 205-217 
Okochi M, Walter J, Koyama A, Nakajo S, Baba M, Iwatsubo T, Meijer L, Kahle PJ, Haass C (2000) Constitutive phosphorylation of the Parkinson's disease associated alpha-synuclein. The Journal of biological chemistry 275(1): 390-397

Oschkinat H, Griesinger C, Kraulis PJ, Sorensen OW, Ernst RR, Gronenborn AM, Clore GM (1988) 3-Dimensional Nmr-Spectroscopy of a Protein in Solution. Nature 332(6162): $374-376$

Ottiger M, Delaglio F, Bax A (1998) Measurement of J and dipolar couplings from simplified two-dimensional NMR spectra. J Magn Reson 131(2): 373-378

Oueslati A, Fournier M, Lashuel HA (2010a) Role of post-translational modifications in modulating the structure, function and toxicity of alpha-synuclein: implications for Parkinson's disease pathogenesis and therapies. Progress in brain research 183: 115145

Oueslati A, Fournier M, Lashuel HA (2010b) Role of post-translational modifications in modulating the structure, function and toxicity of alpha-synuclein: implications for Parkinson's disease pathogenesis and therapies. Recent Advances in Parkinsons Disease: Basic Research 183: 115-145

Outeiro TF, Lindquist S (2003) Yeast cells provide insight into alpha-synuclein biology and pathobiology. Science (New York, NY 302(5651): 1772-1775

Outeiro TF, Putcha P, Tetzlaff JE, Spoelgen R, Koker M, Carvalho F, Hyman BT, McLean PJ (2008) Formation of toxic oligomeric alpha-synuclein species in living cells. PloS one 3(4): e1867

Paleologou KE, Oueslati A, Shakked G, Rospigliosi CC, Kim HY, Lamberto GR, Fernandez CO, Schmid A, Chegini F, Gai WP, Chiappe D, Moniatte M, Schneider BL, Aebischer P, Eliezer D, Zweckstetter M, Masliah E, Lashuel HA Phosphorylation at S87 is enhanced in synucleinopathies, inhibits alpha-synuclein oligomerization, and influences synuclein-membrane interactions. J Neurosci 30(9): 3184-3198

Paleologou KE, Oueslati A, Shakked G, Rospigliosi CC, Kim HY, Lamberto GR, Fernandez CO, Schmid A, Chegini F, Gai WP, Chiappe D, Moniatte M, Schneider BL, Aebischer P, Eliezer D, Zweckstetter M, Masliah E, Lashuel HA (2010) Phosphorylation at S87 is enhanced in synucleinopathies, inhibits alpha-synuclein oligomerization, and influences synuclein-membrane interactions. J Neurosci 30(9): 3184-3198

Paleologou KE, Schmid AW, Rospigliosi CC, Kim HY, Lamberto GR, Fredenburg RA, Lansbury PT, Jr., Fernandez CO, Eliezer D, Zweckstetter M, Lashuel HA (2008) 
Phosphorylation at Ser-129 but not the phosphomimics S129E/D inhibits the fibrillation of alpha-synuclein. The Journal of biological chemistry 283(24): 16895-16905

Pan-Montojo F, Anichtchik O, Dening Y, Knels L, Pursche S, Jung R, Jackson S, Gille G, Spillantini MG, Reichmann H, Funk RH (2010) Progression of Parkinson's disease pathology is reproduced by intragastric administration of rotenone in mice. PloS one 5(1): e8762

Pankratz N, Wojcieszek J, Foroud T (2004) Parkinson Disease Overview: Seattle (WA): University of Washington, Seattle.

Pervushin K, Riek R, Wider G, Wuthrich K (1997) Attenuated T2 relaxation by mutual cancellation of dipole-dipole coupling and chemical shift anisotropy indicates an avenue to NMR structures of very large biological macromolecules in solution. Proceedings of the National Academy of Sciences of the United States of America 94(23): 12366-12371

Poirot O, O'Toole E, Notredame C (2003) Tcoffee@igs: A web server for computing, evaluating and combining multiple sequence alignments. Nucleic acids research 31(13): 3503-3506

Polymeropoulos MH, Lavedan C, Leroy E, Ide SE, Dehejia A, Dutra A, Pike B, Root H, Rubenstein J, Boyer R, Stenroos ES, Chandrasekharappa S, Athanassiadou A, Papapetropoulos T, Johnson WG, Lazzarini AM, Duvoisin RC, Di Iorio G, Golbe LI, Nussbaum RL (1997) Mutation in the alpha-synuclein gene identified in families with Parkinson's disease. Science (New York, NY 276(5321): 2045-2047

Prestegard JH, Bougault CM, Kishore AI (2004) Residual dipolar couplings in structure determination of biomolecules. Chemical reviews 104(8): 3519-3540

Puschmann A, Ross OA, Vilarino-Guell C, Lincoln SJ, Kachergus JM, Cobb SA, Lindquist SG, Nielsen JE, Wszolek ZK, Farrer M, Widner H, van Westen D, Hagerstrom D, Markopoulou K, Chase BA, Nilsson K, Reimer J, Nilsson C (2009) A Swedish family with de novo alpha-synuclein A53T mutation: evidence for early cortical dysfunction. Parkinsonism Relat Disord 15(9): 627-632

Putcha P, Danzer KM, Kranich LR, Scott A, Silinski M, Mabbett S, Hicks CD, Veal JM, Steed PM, Hyman BT, McLean PJ (2010) Brain-permeable small-molecule inhibitors of Hsp90 prevent alpha-synuclein oligomer formation and rescue alpha-synuclein-induced toxicity. The Journal of pharmacology and experimental therapeutics 332(3): 849-857

Rezaei-Ghaleh N, Blackledge M, Zweckstetter M (2012) Intrinsically disordered proteins: from sequence and conformational properties toward drug discovery. Chembiochem 13(7): 930-950 
Roodveldt C, Bertoncini CW, Andersson A, van der Goot AT, Hsu ST, FernandezMontesinos R, de Jong J, van Ham TJ, Nollen EA, Pozo D, Christodoulou J, Dobson CM (2009) Chaperone proteostasis in Parkinson's disease: stabilization of the Hsp70/alpha-synuclein complex by Hip. Embo $J$ 28(23): 3758-3770

Rospigliosi CC, McClendon S, Schmid AW, Ramlall TF, Barre P, Lashuel HA, Eliezer D (2009) E46K Parkinson's-linked mutation enhances C-terminal-to-N-terminal contacts in alpha-synuclein. J Mol Biol 388(5): 1022-1032

Rott R, Szargel R, Haskin J, Shani V, Shainskaya A, Manov I, Liani E, Avraham E, Engelender S (2008) Monoubiquitylation of alpha-synuclein by seven in absentia homolog (SIAH) promotes its aggregation in dopaminergic cells. The Journal of biological chemistry 283(6): 3316-3328

Salmon L, Nodet G, Ozenne V, Yin G, Jensen MR, Zweckstetter M, Blackledge M (2010) NMR characterization of long-range order in intrinsically disordered proteins. Journal of the American Chemical Society 132(24): 8407-8418

Salzmann M, Pervushin K, Wider G, Senn H, Wuthrich K (1998) TROSY in tripleresonance experiments: new perspectives for sequential NMR assignment of large proteins. Proceedings of the National Academy of Sciences of the United States of America 95(23): 13585-13590

Sancenon V, Lee SA, Patrick C, Griffith J, Paulino A, Outeiro TF, Reggiori F, Masliah E, Muchowski PJ (2012) Suppression of alpha-synuclein toxicity and vesicle trafficking defects by phosphorylation at S129 in yeast depends on genetic context. Human molecular genetics 21(11): 2432-2449

Satake W, Nakabayashi Y, Mizuta I, Hirota Y, Ito C, Kubo M, Kawaguchi T, Tsunoda T, Watanabe M, Takeda A, Tomiyama H, Nakashima K, Hasegawa K, Obata F, Yoshikawa T, Kawakami H, Sakoda S, Yamamoto M, Hattori N, Murata M, Nakamura Y, Toda T (2009) Genome-wide association study identifies common variants at four loci as genetic risk factors for Parkinson's disease. Nature genetics 41(12): 1303-1307

Sattler M, Schleucher J, Griesinger C (1999) Heteronuclear multidimensional NMR experiments for the structure determination of proteins in solution employing pulsed field gradients. Progress in Nuclear Magnetic Resonance Spectroscopy 34(2): 93-158

Schanda P, Brutscher B (2005) Very fast two-dimensional NMR spectroscopy for realtime investigation of dynamic events in proteins on the time scale of seconds. Journal of the American Chemical Society 127(22): 8014-8015 
Schanda P, Kupce E, Brutscher B (2005) SOFAST-HMQC experiments for recording two-dimensional heteronuclear correlation spectra of proteins within a few seconds. Journal of biomolecular NMR 33(4): 199-211

Schapira AH, Bezard E, Brotchie J, Calon F, Collingridge GL, Ferger B, Hengerer B, Hirsch E, Jenner P, Le Novere N, Obeso JA, Schwarzschild MA, Spampinato U, Davidai G (2006) Novel pharmacological targets for the treatment of Parkinson's disease. Nature reviews 5(10): 845-854

Scheidt HA, Morgado I, Huster D (2012) Solid-state NMR Reveals a Close Structural Relationship between Amyloid-beta Protofibrils and Oligomers. The Journal of biological chemistry 287(27): 22822-22826

Schlichting I, Almo SC, Rapp G, Wilson K, Petratos K, Lentfer A, Wittinghofer A, Kabsch W, Pai EF, Petsko GA, et al. (1990) Time-resolved X-ray crystallographic study of the conformational change in Ha-Ras p21 protein on GTP hydrolysis. Nature 345(6273): 309-315

Schwarzinger S, Kroon GJ, Foss TR, Chung J, Wright PE, Dyson HJ (2001) Sequencedependent correction of random coil NMR chemical shifts. J Am Chem Soc 123(13): 2970-2978

Schwarzinger S, Kroon GJ, Foss TR, Wright PE, Dyson HJ (2000) Random coil chemical shifts in acidic $8 \mathrm{M}$ urea: implementation of random coil shift data in NMRView. Journal of biomolecular NMR 18(1): 43-48

Simon-Sanchez J, Schulte C, Bras JM, Sharma M, Gibbs JR, Berg D, Paisan-Ruiz C, Lichtner P, Scholz SW, Hernandez DG, Kruger R, Federoff M, Klein C, Goate A, Perlmutter J, Bonin M, Nalls MA, Illig T, Gieger C, Houlden H, Steffens M, Okun MS, Racette BA, Cookson MR, Foote KD, Fernandez HH, Traynor BJ, Schreiber S, Arepalli S, Zonozi R, Gwinn K, van der Brug M, Lopez G, Chanock SJ, Schatzkin A, Park Y, Hollenbeck A, Gao J, Huang X, Wood NW, Lorenz D, Deuschl G, Chen H, Riess O, Hardy JA, Singleton AB, Gasser T (2009) Genome-wide association study reveals genetic risk underlying Parkinson's disease. Nature genetics 41(12): 1308-1312

Sklenar V, Bax A (1987) Spin-Echo Water Suppression for the Generation of PurePhase Two-Dimensional Nmr-Spectra. Journal of Magnetic Resonance 74(3): 469-479

Skora L, Cho MK, Kim HY, Becker S, Fernandez CO, Blackledge M, Zweckstetter M (2006) Charge-induced molecular alignment of intrinsically disordered proteins. Angewandte Chemie (International ed 45(42): 7012-7015 
Smith WW, Margolis RL, Li X, Troncoso JC, Lee MK, Dawson VL, Dawson TM, Iwatsubo T, Ross CA (2005) Alpha-synuclein phosphorylation enhances eosinophilic cytoplasmic inclusion formation in SH-SY5Y cells. J Neurosci 25(23): 5544-5552

Soper JH, Kehm V, Burd CG, Bankaitis VA, Lee VM (2011) Aggregation of alphasynuclein in $\mathrm{S}$. cerevisiae is associated with defects in endosomal trafficking and phospholipid biosynthesis. J Mol Neurosci 43(3): 391-405

Souza JM, Giasson BI, Chen Q, Lee VM, Ischiropoulos H (2000) Dityrosine crosslinking promotes formation of stable alpha -synuclein polymers. Implication of nitrative and oxidative stress in the pathogenesis of neurodegenerative synucleinopathies. The Journal of biological chemistry 275(24): 18344-18349

Spillantini MG, Goedert M (2000) The alpha-synucleinopathies: Parkinson's disease, dementia with Lewy bodies, and multiple system atrophy. Annals of the New York Academy of Sciences 920: 16-27

Spillantini MG, Schmidt ML, Lee VM, Trojanowski JQ, Jakes R, Goedert M (1997) Alpha-synuclein in Lewy bodies. Nature 388(6645): 839-840

Stefani M, Dobson CM (2003) Protein aggregation and aggregate toxicity: new insights into protein folding, misfolding diseases and biological evolution. Journal of molecular medicine (Berlin, Germany) 81(11): 678-699

Steiner JA, Angot E, Brundin P A deadly spread: cellular mechanisms of alphasynuclein transfer. Cell death and differentiation 18(9): 1425-1433

Stenmark H (2009) Rab GTPases as coordinators of vesicle traffic. Nature reviews 10(8): 513-525

Sugeno N, Takeda A, Hasegawa T, Kobayashi M, Kikuchi A, Mori F, Wakabayashi K, Itoyama Y (2008) Serine 129 phosphorylation of alpha-synuclein induces unfolded protein response-mediated cell death. The Journal of biological chemistry 283(34): 23179-23188

Taschenberger G, Garrido M, Tereshchenko Y, Bahr M, Zweckstetter M, Kugler S (2012) Aggregation of alphaSynuclein promotes progressive in vivo neurotoxicity in adult rat dopaminergic neurons. Acta neuropathologica 123(5): 671-683

Tofaris GK, Kim HT, Hourez R, Jung JW, Kim KP, Goldberg AL (2011) Ubiquitin ligase Nedd4 promotes alpha-synuclein degradation by the endosomal-lysosomal pathway. Proceedings of the National Academy of Sciences of the United States of America 108(41): 17004-17009 
Tofaris GK, Layfield R, Spillantini MG (2001) alpha-synuclein metabolism and aggregation is linked to ubiquitin-independent degradation by the proteasome. FEBS letters 509(1): 22-26

Tofaris GK, Razzaq A, Ghetti B, Lilley KS, Spillantini MG (2003) Ubiquitination of alpha-synuclein in Lewy bodies is a pathological event not associated with impairment of proteasome function. The Journal of biological chemistry 278(45): 44405-44411

Touchot N, Zahraoui A, Vielh E, Tavitian A (1989) Biochemical properties of the YPTrelated rab1B protein. Comparison with rab1A. FEBS letters 256(1-2): 79-84

Uversky VN (2009) Intrinsic disorder in proteins associated with neurodegenerative diseases. Front Biosci 14: 5188-5238

Uversky VN, Oldfield CJ, Dunker AK (2008) Intrinsically disordered proteins in human diseases: introducing the D2 concept. Annual review of biophysics 37: 215-246

van Raaij ME, Segers-Nolten IMJ, Subramaniam V (2006) Quantitative morphological analysis reveals ultrastructural diversity of amyloid fibrils from alpha-synuclein mutants. Biophysical Journal 91(11): L96-L98

Vilar M, Chou HT, Luhrs T, Maji SK, Riek-Loher D, Verel R, Manning G, Stahlberg H, Riek R (2008) The fold of alpha-synuclein fibrils. Proceedings of the National Academy of Sciences of the United States of America 105(25): 8637-8642

Visanji NP, Wislet-Gendebien S, Oschipok LW, Zhang G, Aubert I, Fraser PE, Tandon A Effect of Ser-129 phosphorylation on interaction of alpha-synuclein with synaptic and cellular membranes. The Journal of biological chemistry 286(41): 35863-35873

Visanji NP, Wislet-Gendebien S, Oschipok LW, Zhang G, Aubert I, Fraser PE, Tandon A (2011) Effect of Ser-129 phosphorylation on interaction of alpha-synuclein with synaptic and cellular membranes. The Journal of biological chemistry 286(41): 3586335873

Volles MJ, Lansbury PT, Jr. (2003) Zeroing in on the pathogenic form of alphasynuclein and its mechanism of neurotoxicity in Parkinson's disease. Biochemistry 42(26): 7871-7878

Volpicelli-Daley LA, Luk KC, Patel TP, Tanik SA, Riddle DM, Stieber A, Meaney DF, Trojanowski JQ, Lee VM Exogenous alpha-synuclein fibrils induce Lewy body pathology leading to synaptic dysfunction and neuron death. Neuron 72(1): 57-71

Wüthrich K. (1986) NMR of Proteins and Nucleic Acids. Wiley. 
Wang L, Eghbalnia HR, Bahrami A, Markley JL (2005) Linear analysis of carbon-13 chemical shift differences and its application to the detection and correction of errors in referencing and spin system identifications. Journal of biomolecular NMR 32(1): 13-22

Waxman EA, Giasson BI (2011) Characterization of kinases involved in the phosphorylation of aggregated alpha-synuclein. J Neurosci Res 89(2): 231-247

Weinreb PH, Zhen W, Poon AW, Conway KA, Lansbury PT, Jr. (1996) NACP, a protein implicated in Alzheimer's disease and learning, is natively unfolded. Biochemistry 35(43): 13709-13715

Wider G, Dreier L (2006) Measuring Protein Concentrations by NMR Spectroscopy. Journal of the American Chemical Society 128: 2571-2576

Wilson BS, Nuoffer C, Meinkoth JL, McCaffery M, Feramisco JR, Balch WE, Farquhar MG (1994) A Rab1 mutant affecting guanine nucleotide exchange promotes disassembly of the Golgi apparatus. The Journal of cell biology 125(3): 557-571

Winklhofer KF, Tatzelt J, Haass C (2008) The two faces of protein misfolding: gainand loss-of-function in neurodegenerative diseases. The EMBO journal 27(2): 336-349

$\mathrm{Wu} \mathrm{KP}$, Baum $\mathrm{J}$ Detection of transient interchain interactions in the intrinsically disordered protein alpha-synuclein by NMR paramagnetic relaxation enhancement. Journal of the American Chemical Society 132(16): 5546-5547

Zarranz JJ, Alegre J, Gomez-Esteban JC, Lezcano E, Ros R, Ampuero I, Vidal L, Hoenicka J, Rodriguez O, Atares B, Llorens V, Gomez Tortosa E, del Ser T, Munoz DG, de Yebenes JG (2004) The new mutation, E46K, of alpha-synuclein causes Parkinson and Lewy body dementia. Ann Neurol 55(2): 164-173

Zheng G, Stait-Gardner T, Anil Kumar PG, Torres AM, Price WS (2008) PGSTEWATERGATE: an STE-based PGSE NMR sequence with excellent solvent suppression. J Magn Reson 191(1): 159-163

Zibaee S, Jakes R, Fraser G, Serpell LC, Crowther RA, Goedert M (2007) Sequence Determinants for Amyloid Fibrillogenesis of Human alpha-Synuclein. Journal of molecular biology 374(2): 454-464

Zweckstetter M (2008) NMR: prediction of molecular alignment from structure using the PALES software. Nature protocols 3(4): 679-690 


\section{Appendix}

\section{Backbone assignment of Rab1b(1-175)-GDP}

\begin{tabular}{|c|c|c|c|c|c|}
\hline \multirow{2}{*}{\multicolumn{6}{|c|}{$\begin{array}{l}\text { RES } \\
\text { GLY_1 }\end{array}$}} \\
\hline & & & & & \\
\hline \multicolumn{6}{|l|}{ HIS_z } \\
\hline \multicolumn{6}{|l|}{ MET_3 } \\
\hline \multicolumn{6}{|l|}{ PRO_4 } \\
\hline GLU_5 & 120.9 & 8.492 & 175.3 & 57.1 & 30.24 \\
\hline TYR_6 & 113.7 & 7.042 & 173.6 & 55.72 & 38.81 \\
\hline ASP_7 & 119.3 & 8.964 & 175.4 & 56.74 & 43.51 \\
\hline TYR_8 & 114.8 & 7.703 & 172.7 & 56.45 & \\
\hline LEU_9 & 125 & 8.325 & & 54.04 & 43.92 \\
\hline PHE_10 & 123.9 & 8.662 & 174.1 & 51.08 & 40.71 \\
\hline LYS_11 & 128.5 & 11.64 & 175.8 & 55.8 & \\
\hline LEU_12 & 127.4 & 9.556 & & 52.63 & 46.07 \\
\hline LEU_13 & 119.1 & 8.064 & & 53.51 & \\
\hline \multicolumn{6}{|l|}{ LEU_14 } \\
\hline \multicolumn{6}{|l|}{ ILE_- 15} \\
\hline \multicolumn{6}{|l|}{ GLY_16 } \\
\hline ASP_17 & 122.4 & 9.038 & 176.9 & 55.85 & 40.81 \\
\hline SER_18 & 115.5 & 8.805 & 176.1 & 60.33 & 63.44 \\
\hline GLY_19 & 116.4 & 11.17 & 174.5 & 45.82 & \\
\hline VAL_20 & 123.8 & 10.43 & 178.3 & 61.04 & 29.68 \\
\hline \multicolumn{6}{|l|}{ GLY_21 } \\
\hline \multicolumn{6}{|l|}{ LYS_22 } \\
\hline SER_23 & 117.8 & 8.333 & & 61.49 & 55.27 \\
\hline CYS_24 & 120.9 & 9.648 & 179.1 & 64.64 & 26.84 \\
\hline LEU_25 & 125.9 & 9.286 & 177.9 & 59.47 & 42.7 \\
\hline LEU_26 & 119.3 & 7.979 & & 59.01 & 41.84 \\
\hline LEU_27 & 119 & 8.82 & 180.1 & 58.47 & 41.3 \\
\hline ARG_28 & 119 & 8.514 & 178 & 57.77 & 27.42 \\
\hline PHE_29 & 119 & 8.198 & 178.4 & 60.17 & 40.52 \\
\hline \multicolumn{6}{|l|}{ ALA_30 } \\
\hline ASP_31 & 113.9 & 7.734 & & 53.95 & 42.75 \\
\hline ASP_32 & 120.5 & 7.751 & 174.6 & 55.24 & 40.01 \\
\hline THR_33 & 106.9 & 7.048 & 173 & 59.59 & 71.75 \\
\hline TYR_34 & 119 & 8.062 & 173.3 & 58.88 & 41.61 \\
\hline THR_35 & 122.7 & 7.447 & 171 & 58.96 & 70.13 \\
\hline GLU_36 & 122.8 & 8.251 & 177 & 56.36 & 29.53 \\
\hline SER_37 & 117.2 & 7.934 & 173 & 58.64 & 63.6 \\
\hline
\end{tabular}




\begin{tabular}{|c|c|c|c|c|c|}
\hline TYR_38 & 123.3 & 8.314 & 175.7 & 58.78 & 38.3 \\
\hline ILE_39 & 129.8 & 8.554 & 175.2 & 60.05 & 38.95 \\
\hline \multicolumn{6}{|l|}{ SER_40 } \\
\hline \multicolumn{6}{|l|}{ THR_41 } \\
\hline \multicolumn{6}{|l|}{ ILE_42 } \\
\hline GLY_43 & 111.6 & 8.511 & 172.7 & 45.74 & \\
\hline VAL_44 & 113.7 & 7.21 & 175.1 & 59.66 & 35 \\
\hline \multicolumn{6}{|l|}{ ASP 45} \\
\hline PHE_46 & 109.5 & 6.54 & 173.7 & 54.27 & 40.47 \\
\hline LYS_47 & 116.4 & 8.451 & 174.6 & 53.87 & 36.53 \\
\hline ILE_48 & 118.9 & 8.534 & 176.1 & 58.54 & 40.72 \\
\hline ARG_49 & 125 & 8.464 & 174.1 & 55.63 & 34.1 \\
\hline THR_50 & 123.2 & 8.733 & & 62 & 69.21 \\
\hline ILE_51 & 121 & 9.223 & 173.3 & 59.16 & 42.29 \\
\hline GLU_52 & 121.2 & 8.31 & & 54.66 & 32.07 \\
\hline LEU_53 & 124.5 & 8.734 & 176 & 54.44 & 45.95 \\
\hline ASP_54 & 124.6 & 9.385 & & 55.16 & 39.88 \\
\hline GLY_55 & 104.2 & 8.87 & 173.9 & 45.47 & \\
\hline LYS_56 & 119.9 & 8.048 & 175.4 & 52.74 & 33.7 \\
\hline THR_57 & 119.6 & 9.87 & 174.6 & 63 & 69.41 \\
\hline ILE_58 & 129.7 & 9.416 & & 59.62 & 40.2 \\
\hline LYS_59 & 128.9 & 8.812 & & 55.81 & 33.74 \\
\hline LEU_60 & 125.4 & 8.743 & & 52.95 & 43.01 \\
\hline GLN_61 & 124.7 & 8.971 & & 54.66 & 30.96 \\
\hline ILE_62 & 126.8 & 9.185 & & 61.28 & 39.44 \\
\hline TRP_63 & 128 & 9.199 & & 58.2 & \\
\hline ASP_64 & 120.7 & 8.155 & & 52.17 & 45.15 \\
\hline \multicolumn{6}{|l|}{ THR_65 } \\
\hline \multicolumn{6}{|l|}{ ALA_66 } \\
\hline \multicolumn{6}{|l|}{ GLY_67 } \\
\hline \multicolumn{6}{|l|}{ GLN_68 } \\
\hline \multicolumn{6}{|l|}{ GLU_69 } \\
\hline \multicolumn{6}{|l|}{ ARG_70 } \\
\hline \multicolumn{6}{|l|}{ PHE_71 } \\
\hline \multicolumn{6}{|l|}{ ARG_72 } \\
\hline \multicolumn{6}{|l|}{ THR_73 } \\
\hline \multicolumn{6}{|l|}{ ILE_74 } \\
\hline \multicolumn{6}{|l|}{ THR_75 } \\
\hline SER_76 & 129.7 & 9.375 & & 59.55 & \\
\hline SER_77 & 113.4 & 7.653 & & 59.11 & \\
\hline \multicolumn{6}{|l|}{ TYR_78 } \\
\hline TYR_79 & 129.3 & 9.103 & & 60.96 & 31.93 \\
\hline \multicolumn{6}{|l|}{ ARG_80 } \\
\hline \multicolumn{6}{|l|}{ GLY_81 } \\
\hline ALA_82 & 120.2 & 7.129 & 177.2 & 52.68 & 18.04 \\
\hline HIS_83 & 122.1 & 9.697 & 175.8 & 59.8 & 33.37 \\
\hline GLY_84 & 103.4 & 7.679 & 171.3 & 45.4 & \\
\hline
\end{tabular}




\begin{tabular}{|c|c|c|c|c|c|}
\hline ILE_85 & 122.9 & 8.876 & 173.2 & 60.26 & 41.42 \\
\hline \multicolumn{6}{|l|}{ ILE_86 } \\
\hline \multicolumn{6}{|l|}{ VAL_87 } \\
\hline VAL_88 & 127.1 & 8.84 & 175.3 & & \\
\hline TYR_89 & 122.3 & 9.094 & 170.5 & 55.55 & 40.01 \\
\hline ASP_90 & 121.5 & 8.856 & & 51.31 & 43.41 \\
\hline VAL_91 & 118.5 & 8.722 & 176.3 & 64.24 & 30.82 \\
\hline THR_92 & 113 & 9.481 & 173.4 & 61.9 & 70.05 \\
\hline ASP_93 & 124 & 8.288 & & 52.51 & 43.14 \\
\hline GLN_94 & 126.8 & 9.175 & 178 & 59.04 & 28.33 \\
\hline GLU_95 & 120.6 & 8.291 & & 59.62 & 28.61 \\
\hline SER_96 & 116.1 & 7.959 & 176.2 & 61.27 & 63.46 \\
\hline TYR_97 & 123 & 7.188 & 178.5 & 60.13 & 39.21 \\
\hline ALA_98 & 126.4 & 8.666 & 181.2 & 55.28 & 17.55 \\
\hline ASN_99 & 113.9 & 8.374 & 177.4 & 54.14 & 38.15 \\
\hline VAL_100 & 123.2 & 7.946 & & 67.77 & 30.9 \\
\hline LYS_101 & 116 & 7.378 & 178.7 & 60.62 & 31.99 \\
\hline GLN_102 & 119.2 & 7.265 & 179.1 & 58.64 & 27.84 \\
\hline TRP_103 & 121.5 & 7.83 & 178.8 & 60.46 & 29.43 \\
\hline LEU_104 & 117.7 & 8.758 & 179.1 & 58.36 & 40.79 \\
\hline GLN_105 & 118.6 & 7.49 & 178.6 & 58.96 & 28.39 \\
\hline GLU_106 & 121.1 & 7.753 & 178 & 59.99 & 29.76 \\
\hline ILE_107 & 118.8 & 7.984 & & 65.88 & 38.25 \\
\hline ASP_108 & 118.2 & 7.72 & & 56.99 & 40.39 \\
\hline ARG_109 & 117.1 & 7.385 & 178.3 & 58.55 & 30.54 \\
\hline TYR_110 & 113.9 & 7.488 & 176.3 & 57.7 & 41.31 \\
\hline ALA_111 & 121.8 & 8.629 & 176.6 & 51.2 & 21.61 \\
\hline SER_112 & 115 & 8.707 & 175.6 & 59.16 & 64 \\
\hline GLU_113 & 123.8 & 8.995 & 176.8 & 58.59 & 29.48 \\
\hline ASN_114 & 115.4 & 8.304 & 174.3 & 52.83 & 38.18 \\
\hline VAL_115 & 118.7 & 7.057 & 174.2 & 62.44 & 31.98 \\
\hline ASN_116 & 124.2 & 7.717 & 174.6 & 55.84 & 41.01 \\
\hline LYS_117 & 120.1 & 8.418 & & 55.13 & 37.06 \\
\hline LEU_118 & 120.4 & 7.673 & 172.8 & 55.18 & 47.26 \\
\hline LEU_119 & 128.2 & 8.656 & & 53.74 & 45.03 \\
\hline VAL_120 & 125.7 & 9.325 & 174 & 59.55 & 36.06 \\
\hline GLY_121 & 112.3 & 8.105 & 171.9 & 44.42 & \\
\hline ASN_122 & 123.1 & 9.075 & 174 & 51.52 & 41.47 \\
\hline LYS_123 & 111.8 & 7.232 & 177.6 & 57.56 & 29.26 \\
\hline SER_124 & 113.9 & 8.933 & 173.8 & 60.7 & 63.15 \\
\hline ASP_125 & 116.1 & 8.495 & 176.8 & 54.36 & 41.18 \\
\hline LEU_126 & 120.7 & 7.829 & & & \\
\hline THR_127 & 112.4 & 7.809 & 177.6 & 65.87 & 68.4 \\
\hline \multicolumn{6}{|l|}{ THR_128 } \\
\hline LYS_129 & 119.2 & 7.278 & 175.1 & 54.75 & 33.61 \\
\hline LYS_130 & 121.2 & 7.01 & 176.2 & 58.89 & 33.56 \\
\hline VAL_131 & 119.7 & 8.768 & 175.2 & 61.34 & 33.8 \\
\hline
\end{tabular}




\begin{tabular}{|c|c|c|c|c|c|}
\hline VAL_132 & 123.4 & 7.548 & & 61.69 & 31.66 \\
\hline ASP_133 & 129.3 & 8.582 & 176.6 & 54.75 & 42.02 \\
\hline \multicolumn{6}{|l|}{ ASN_134 } \\
\hline THR_135 & 115.6 & 8.396 & 176.5 & 66.18 & 68.09 \\
\hline THR_136 & 120.1 & 7.365 & 176.1 & 66.05 & 68.73 \\
\hline ALA_137 & 125.1 & 7.186 & 178.5 & 54.74 & 20.21 \\
\hline LYS_138 & 109.4 & 7.712 & & 59.35 & \\
\hline GLU_139 & 118.4 & 7.835 & 179.8 & 59.68 & 29.21 \\
\hline PHE_140 & 121.6 & 7.788 & 178.2 & 59.88 & 38.97 \\
\hline ALA_141 & 125.4 & 8.877 & 180.3 & 56.11 & 18.15 \\
\hline ASP_142 & 118.5 & 9.335 & & 57.29 & 39.74 \\
\hline SER_143 & 118.2 & 8.003 & & 61.51 & 62.46 \\
\hline LEU_144 & 121.5 & 7.167 & 177.4 & 54.51 & 43.79 \\
\hline GLY_145 & 109.7 & 7.989 & 174.9 & 46.27 & \\
\hline ILE_146 & 116 & 7.81 & 172.8 & 58.02 & 39.56 \\
\hline \multicolumn{6}{|l|}{ PRO_147 } \\
\hline PHE_148 & 119.7 & 7.877 & 174.7 & 55.31 & 43.89 \\
\hline LEU_149 & 128.2 & 8.015 & 173.5 & 54.7 & 47.07 \\
\hline GLU_150 & 117.3 & 7.992 & 177.5 & 54.39 & 32.31 \\
\hline THR_151 & 113.3 & 9.256 & 175.1 & 60.01 & 73.74 \\
\hline SER_152 & 111.4 & 8.19 & 175.8 & 56.8 & 64.47 \\
\hline ALA_153 & 133.3 & 9.043 & 178.1 & 56.15 & 18.16 \\
\hline LYS_154 & 117.8 & 7.104 & 176.8 & 58.46 & 33.91 \\
\hline ASN_155 & 114.2 & 7.956 & 175.2 & 52.21 & 38.05 \\
\hline ALA_156 & 118.8 & 7.744 & 176.2 & 55.08 & 17.31 \\
\hline THR_157 & 117.3 & 8.083 & 174.9 & 64.44 & 68.96 \\
\hline ASN_158 & 120.9 & 9.358 & 174.5 & 54.48 & 37.01 \\
\hline VAL_159 & 121.9 & 7.664 & 177.1 & 68.48 & 30.75 \\
\hline GLU_160 & 119.4 & 8.746 & 178.8 & 60.57 & 29.17 \\
\hline GLN_161 & 116.8 & 8.483 & 178.5 & 59.23 & 27.58 \\
\hline ALA_162 & 124.2 & 8.172 & 177.9 & 55.82 & 16.55 \\
\hline PHE_163 & 113.9 & 7.014 & 178.2 & 62.92 & 39.95 \\
\hline MET_164 & 117.8 & 8.831 & 179.2 & 58.22 & 31.12 \\
\hline THR_165 & 118.2 & 8.557 & & 67.12 & 68.35 \\
\hline MET_166 & 119.8 & 7.465 & 176.9 & 58.28 & 32.33 \\
\hline ALA_167 & 119.6 & 8.384 & 178.1 & 55.73 & 18.82 \\
\hline ALA_168 & 119.2 & 8.338 & 180.9 & 55.25 & 18.33 \\
\hline GLU_169 & 119.1 & 8.165 & 179.3 & 59.14 & 29.39 \\
\hline ILE_170 & 120.8 & 7.917 & 178.3 & 64.66 & 38.64 \\
\hline LYS_171 & 118.9 & 8.208 & & 60.22 & 32.2 \\
\hline LYS_172 & 117.9 & 7.721 & 178 & 59.31 & 32.55 \\
\hline ARG_173 & 117.2 & 7.466 & 177.4 & 57.36 & 30.38 \\
\hline MET_174 & 118.2 & 8.003 & & 56.12 & 32.94 \\
\hline GLY_175 & 113.8 & 7.861 & 179.3 & 46.49 & \\
\hline
\end{tabular}




\section{Backbone assignment of Rab8a-GDP}

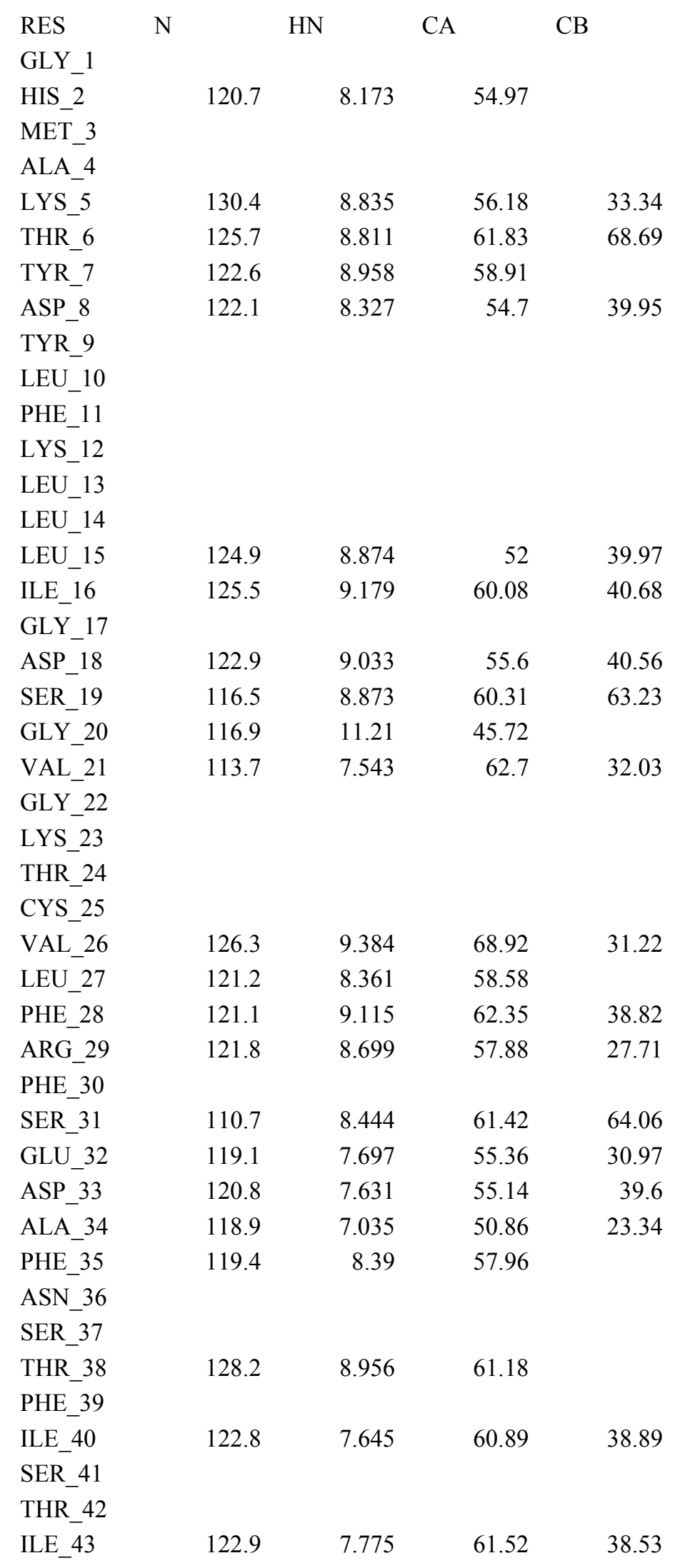




\begin{tabular}{|c|c|c|c|c|}
\hline GLY_44 & 114 & 8.486 & 45.68 & \\
\hline ILE_45 & 121.3 & 7.775 & 59.84 & 38.38 \\
\hline ASP_46 & 125.4 & 8.259 & 55.43 & 42.06 \\
\hline \multicolumn{5}{|l|}{ PHE_47 } \\
\hline LYS_48 & 125.1 & 8.318 & 56.35 & 33.8 \\
\hline \multicolumn{5}{|l|}{ ILE_49 } \\
\hline \multicolumn{5}{|l|}{ ARG_50 } \\
\hline \multicolumn{5}{|l|}{ THR_51 } \\
\hline \multicolumn{5}{|l|}{ ILE_52 } \\
\hline \multicolumn{5}{|l|}{ GLU_53 } \\
\hline \multicolumn{5}{|l|}{ LEU_54 } \\
\hline ASP_55 & 124.1 & 9.305 & 55.12 & 39.73 \\
\hline GLY_56 & 104.5 & 8.756 & 45.46 & \\
\hline LYS_57 & 120.4 & 7.979 & 52.77 & 34.21 \\
\hline ARG_58 & 122.5 & 9.749 & 55.76 & \\
\hline ILE_59 & 129 & 9.456 & 59.35 & 38.73 \\
\hline \multicolumn{5}{|l|}{ LYS_60 } \\
\hline \multicolumn{5}{|l|}{ LEU_61 } \\
\hline \multicolumn{5}{|l|}{ GLN_62 } \\
\hline \multicolumn{5}{|l|}{ ILE_63 } \\
\hline \multicolumn{5}{|l|}{ TRP_64 } \\
\hline \multicolumn{5}{|l|}{ ASP_65 } \\
\hline \multicolumn{5}{|l|}{ THR_66 } \\
\hline \multicolumn{5}{|l|}{ ALA_67 } \\
\hline \multicolumn{5}{|l|}{ GLY_68 } \\
\hline \multicolumn{5}{|l|}{ GLN_69 } \\
\hline \multicolumn{5}{|l|}{ GLU_70 } \\
\hline \multicolumn{5}{|l|}{ ARG_71 } \\
\hline \multicolumn{5}{|l|}{ PHE_72 } \\
\hline \multicolumn{5}{|l|}{ ARG_73 } \\
\hline \multicolumn{5}{|l|}{ THR_74 } \\
\hline \multicolumn{5}{|l|}{ ILE_75 } \\
\hline THR_76 & & & & \\
\hline THR_77 & & & & \\
\hline ALA_78 & 118.4 & 8.409 & 55.66 & 18.73 \\
\hline TYR_79 & & & & \\
\hline TYR_80 & 125.5 & 8.699 & 60.08 & 40.5 \\
\hline ARG_81 & & & & \\
\hline GLY_82 & & & & \\
\hline ALA_83 & 121.7 & 7.718 & 53.13 & 18.17 \\
\hline MET_84 & 119.9 & 9.069 & 56.17 & \\
\hline GLY_85 & 106.4 & 7.561 & 45.18 & \\
\hline ILE_86 & 124.3 & 9.024 & 60.11 & \\
\hline MET_87 & 124.8 & 8.671 & 53.16 & \\
\hline LEU_88 & & & & \\
\hline VAL_89 & 123.5 & 8.701 & 60.78 & 33.51 \\
\hline TYR_90 & 123.5 & 9.057 & 55.38 & 39.52 \\
\hline
\end{tabular}


ASP 9

ILE_92

$\begin{array}{lll}121.3 & 8.817 \quad 64.78\end{array}$

THR 93

$114.5 \quad 9.379$

61.82

69.89

ASN_94

120.3

8.194

51.14

39.48

GLU_95

128.1

9.549

59.56

29.94

LYS_96

SER_97

$115.6 \quad 7.458$

$61.12 \quad 63.99$

PHE_98

125.4

59.7

40.11

ASP_99

123.2

7.083

57.45

40.29

ASN_100

114.8

7.916

54.15

38.41

ILE_101

124.5

8.008

62.72

34.06

ARG_102

ASN_103

TRP_104

ILE_105

119.5

8.534

65.82

37.13

ARG_106

113.2

7.452

61.85

ASN_107

ILE 108

GLU_109

120.2

7.236

59.17

29.46

GLU_110

116.9

7.573

58.46

30.05

HIS_111

114

7.308

57.41

33.34

ALA_112

122.9

8.466

50.98

20.59

SER_113

115.5

8.417

58.59

63.75

127.1

8.68

54.21

18.68

ASP 115

114.9

8.042

53.5

40.77

VAL_116

121

7.138

$62.7 \quad 32.52$

GLU_117

127.4

7.435

55.42

31.46

LYS_118

122.6

8.611

54.68

35.95

MET_119

ILE_120

118.6

8.319

58.5

40.99

LEU_121

GLY_122

ASN_123

123.1

8.697

52.28

40.97

LYS_124

111.1

7.044

57.7

115.3

8.716

59.77

ASP_126

117.4

8.713

54.12

27.05

VAL_127

123.4

7.877

$60.45 \quad 28.82$

ASN_128

ASP_129

112.3

6.923

54.9

40.6

LYS_130

129.7

7.689

56.56

ARG_131

GLN_132

VAL_133

SER_134

LYS_135

GLU_136

ARG_137

120.6
125.7
127.3
116.6
120.8

7.537
8.462
9.017
8.6
7.648

61.01

33.35

$58.17 \quad 63.8$

$60.24 \quad 32.49$

$59.82 \quad 28.83$

$59.74 \quad 30.56$ 


\begin{tabular}{|c|c|c|c|c|}
\hline GLY_138 & 109.9 & 7.287 & 47.92 & \\
\hline GLU_139 & & & & \\
\hline LYS_140 & 125.3 & 9.38 & 58.54 & 31.95 \\
\hline LEU_141 & 121.7 & 7.473 & 58.44 & 41.48 \\
\hline ALA_142 & 119.6 & 7.655 & 55.66 & 19.03 \\
\hline LEU_143 & 120.4 & 8.108 & 58.09 & 41.86 \\
\hline ASP_144 & 121.9 & 8.327 & 57.24 & \\
\hline TYR_145 & 116 & 7.437 & 59.07 & \\
\hline GLY_146 & 112.8 & 8.176 & 46.85 & \\
\hline ILE_147 & 115.6 & 8.018 & 59.73 & 40.91 \\
\hline LYS_148 & & & & \\
\hline PHE_149 & & & & \\
\hline MET_150 & & & & \\
\hline GLU_151 & 119.8 & 8.261 & 55.78 & 30.57 \\
\hline THR_152 & & & & \\
\hline SER_153 & 112.1 & 8.761 & 57.27 & 65.09 \\
\hline ALA_154 & 134.1 & 9.023 & 55.34 & 18.87 \\
\hline LYS_155 & 120 & 7.07 & 58.85 & 34.58 \\
\hline ALA_156 & 118.3 & 8.123 & 51.14 & 19.04 \\
\hline ASN_157 & 116.8 & 7.208 & 53.18 & 36.26 \\
\hline ILE_158 & 119.1 & 8.239 & 60.55 & 40 \\
\hline ASN_159 & & & & \\
\hline VAL_160 & 113.9 & 6.975 & 69.17 & \\
\hline GLU_161 & & & & \\
\hline ASN_162 & 118 & 7.156 & 55.59 & 37.54 \\
\hline ALA_163 & 125.2 & 8.149 & 56.48 & 18.34 \\
\hline PHE_164 & 114.9 & 7.203 & 63.19 & 40.96 \\
\hline PHE_165 & 118.9 & 9.079 & 62.25 & 37.94 \\
\hline THR_166 & 117 & 8.169 & 67.47 & \\
\hline LEU_167 & & & & \\
\hline ALA_168 & & & & \\
\hline ARG_169 & 119.5 & 8.395 & 60.39 & 29.88 \\
\hline $\mathrm{ASP} \_170$ & 123 & 8.081 & 57.49 & 39.04 \\
\hline ILE_171 & 122.4 & 8.199 & 65.56 & \\
\hline LYS_172 & & & & \\
\hline ALA_173 & 120.2 & 8.268 & 55.1 & 18.09 \\
\hline LYS_174 & 118 & 7.205 & 59.16 & 32.16 \\
\hline MET_175 & & & & \\
\hline $\mathrm{ASP} \_176$ & 120.8 & 8.884 & 56.77 & 39.72 \\
\hline LYS_177 & 119.7 & 7.493 & 58.1 & 32.41 \\
\hline LYS_178 & 120.1 & 7.651 & 58 & \\
\hline LEU_179 & 120.8 & 7.887 & 55.92 & 42.12 \\
\hline GLU_180 & 120.8 & 7.958 & 57.38 & 30.1 \\
\hline GLY_181 & 109.1 & 8.147 & 45.52 & \\
\hline ASN_182 & & & & \\
\hline SER_183 & & & & \\
\hline PRO_184 & & & & \\
\hline
\end{tabular}


GLN 185

GLY 186

SER_187

ASN_188

GLN_189

GLY 190

VAL 191

LYS 192

ILE 193

THR_194

PRO_195

ASP 196

GLN 197

GLN_198

LYS 199

ARG_200

SER_201

SER 202

PHE 203

PHE 204

ARG 205

CYS_206

VAL_207

LEU_208

LEU_209

$\begin{array}{rrrr}126.5 & 8.352 & 56.03 & \\ 124 & 8.195 & 60.72 & 42.03\end{array}$

$\begin{array}{lll}121.3 & 8.221 & 59.7\end{array}$

$\begin{array}{lll}120.6 & 8.061 & 55.72\end{array}$ 


\section{Publications}

Mbefo MK, Paleologou KE, Boucharaba A, Oueslati A, Schell H, Fournier M, Olschewski D, Yin G, Zweckstetter M, Masliah E, Kahle PJ, Hirling H, Lashuel HA, Phosphorylation of synucleins by members of the Polo-like kinase family., J. Biol. Chem. 285:2807-22. 2010.

Di Giovanni S, Eleuteri S, Paleologou KE, Yin G, Zweckstetter M, Carrupt PA, Lashuel HA., Entacapone and tolcapone, two catechol O-methyltransferase inhibitors, block fibril formation of alpha-synuclein and beta-amyloid and protect against amyloidinduced toxicity, J. Biol. Chem. 285, 14941-53, 2010.

Salmon L, Nodet G, Ozenne V, Yin G, Jensen MR, Zweckstetter M, Blackledge M., NMR characterization of long-range order in intrinsically disordered proteins, $J \mathrm{Am}$ Chem Soc. 132: 8407-18. 2010.

Paleologou KE*, Mbefo MK, Yin G*, Oueslati A, Fares MB, Zweckstetter M, Lashuel HA. The PD mutant E46K, but not A30P or A53T, enhances alpha-Synuclein phophorylation at S129, Submitted. *Equal contribution 